\title{
New techniques for quantification of biomarkers and metabolites by magnetic resonance imaging and spectroscopy
}

\author{
Jenkins, Christopher W.
}

How to cite:

Jenkins, Christopher W. (2019) New techniques for quantification of biomarkers and metabolites by magnetic resonance imaging and spectroscopy. Doctoral thesis, Swansea University.

http://cronfa.swan.ac.uk/Record/cronfa50804

Use policy:

This item is brought to you by Swansea University. Any person downloading material is agreeing to abide by the terms of the repository licence: copies of full text items may be used or reproduced in any format or medium, without prior permission for personal research or study, educational or non-commercial purposes only. The copyright for any work remains with the original author unless otherwise specified. The full-text must not be sold in any format or medium without the formal permission of the copyright holder. Permission for multiple reproductions should be obtained from the original author.

Authors are personally responsible for adhering to copyright and publisher restrictions when uploading content to the repository.

Please link to the metadata record in the Swansea University repository, Cronfa (link given in the citation reference above.)

http://www.swansea.ac.uk/library/researchsupport/ris-support/ 


\section{New techniques for quantification of biomarkers and metabolites by magnetic resonance imaging and spectroscopy.}

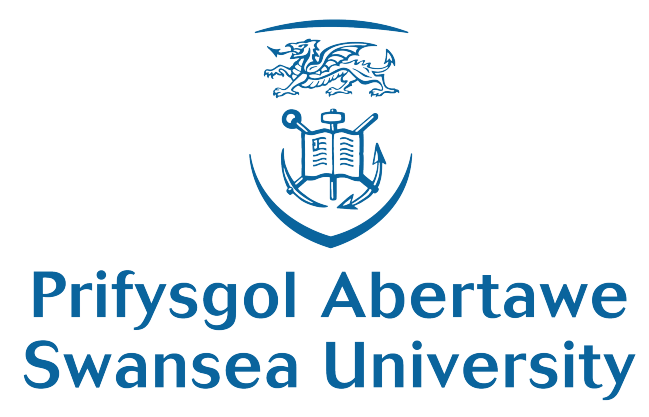

College of Science

Swansea University

This thesis is submitted for the degree of doctor of philosophy.

Chris Jenkins

March 28, 2019 


\begin{abstract}
Since its early beginnings, almost five decades ago, MRI has revolutionised medical imaging, sustaining an active field of research into new applications, and improved understanding of the underlying mechanisms. Its complexity and flexibility, as a noninvasive imaging modality is simultaneously, an asset and a challenge. Quantitative imaging provides a particular challenge due to an increased sensitivity to experimental variations. The development of accurate and robust methods for quantitative magnetic resonance requires protocols to be carefully calibrated to produce consistent results. This necessitates the use of test objects with known, stable, configurable characteristics. This thesis is aimed at the development of these test objects, and their use within quantitative imaging, spectroscopy, and the development of new techniques.

First, a set of magnetic resonance test objects were created, and their relaxation properties assessed. $T_{1}$ and $T_{2}$ are calculated using spin, and multi-spin echo sequences respectively. Several contrast and gelling agents were assessed, and the relaxivity estimated in each case. The protocol dependence of $T_{1}$ estimation methods is examined using a phantom and in-vivo study. Saturation and inversion recovery estimations are compared to variable flip angle methods, and the statistical distributions of $T_{1}$ maps quantified. A series of calibrated phantom studies are conducted, assessing the analysis methods used for in-vivo magnetic resonance spectroscopy. The concentration of brain metabolites is varied within liquid and gel phantoms, and the ratio of GABA to NAA is calculated using a number of analysis tools, and in-house software.

Finally, a magnetic resonance spectroscopy Hamiltonian simulator is implemented in Matlab. The simulator is utilised by collaborators in developing a quantum control framework. Optimal control is used to generate chemically selective RF pulses, and initial experimental implementations explored.

The quantitative methods were found to exhibit both acquisition and analysis method dependencies. However, results were largely consistent within methodology, highlighting the need for consistency across sites to ensure valid comparison. The theoretical development of novel RF pulses has been successful, but much work remains to approach experimental implementation.
\end{abstract}




\section{Declaration}

This work has not previously been accepted in substance for any degree and is not being concurrently submitted in candidature for any degree.

Signed ........................................................ Date

This thesis is the result of my own investigations, except where otherwise stated. Other sources are acknowledged by footnotes giving explicit references. A bibliography is appended.

Signed

Date

I hereby give consent for my thesis to be available for photocopying and for interlibrary loan, and for the title and summary to be made available to outside organisations.

Signed

Date

Chris Jenkins

March 28, 2019 


\section{Acknowledgements}

First, and foremost, I must acknowledge my supervisor, Sophie Shermer. Her contagious enthusiasm for science, and relentless work ethic has been an inspiration to me these past years. Without her, my $\mathrm{PhD}$, and thesis would not have been possible. I am eternally grateful.

To my colleagues and collaborators for their contributions, large and small; Max Chandler, Frank Langbein, Ioannis Papadopoulos, Gizem Portakal, Jonathan Philips, Richard Hugtenberg, Rhodri Evans, and many more.

I would also like to express my gratitude for the funding supplied by Swansea University College of Science, NHS Wales, EPSRC, and The Royal Society.

To my loving family; Lesley, Alan, Daniel, Charlie, and Cassie, without whom, I wouldn't be where I am today. Thank you for your unwavering love and support.

Finally, to my partner Kajal. The months of writing would have been unbearable without you to rant with. I love you. I would be utterly lost without you. 


\section{Contents}

List of Figures $\quad$ VIII

$\begin{array}{ll}\text { List of Tables } & \text { XI }\end{array}$

1 Introduction $\quad 1$

2 Theoretical background $\quad 4$

2.1 Basics of magnetic resonance . . . . . . . . . . . . . . . . 4

2.2 Quantum mechanical description .................. 5

2.3 Macroscopic magnetisation . . . . . . . . . . . . . . . . . . . 6

2.4 Relaxation . . . . . . . . . . . . . . . . . . 8

2.4.1 Longitudinal relaxation $-T_{1} \ldots \ldots \ldots \ldots$

2.4 .2 Transverse relaxation $-T_{2} \ldots \ldots \ldots \ldots$

2.5 Image formation . . . . . . . . . . . . . . . . . . . . 10

2.5.1 Localisation . . . . . . . . . . . . . . . . . . . . 10

2.5.2 Frequency encode gradients .................. 11

2.5.3 Phase encoding gradients . . . . . . . . . . . . . . . . . 12

2.5.4 Pulse sequences . . . . . . . . . . . . . . . 13

2.6 The Bloch equations . . . . . . . . . . . . . . . . 15

2.7 Magnetic resonance spectroscopy _ . . . . . . . . . . . . . . . . . . . 18

2.7.1 Chemical shift . . . . . . . . . . . . . . . . . . . 18

2.7.2 Spin-spin coupling . . . . . . . . . . . . . . . 19

2.7.3 Common sequences . . . . . . . . . . . . . . . 22

3 Test objects for quantitative imaging $\quad 28$

3.1 Introduction . . . . . . . . . . . . . . . . . . . . . . . 28

3.2 Materials and Methods . . . . . . . . . . . . . . . . . . 29

3.2.1 Phantom preparation . . . . . . . . . . . . . . 29

3.2 .2 Scan protocols . . . . . . . . . . . . . . . . . 31

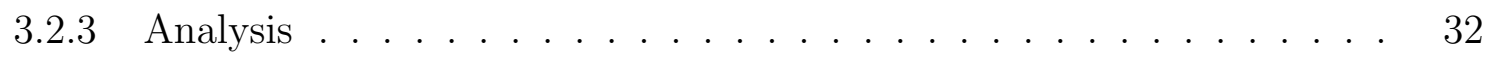


3.3 Results . . . . . . . . . . . . . . . . . . . . . . . . . . 34

3.3 .1 Gelling agents . . . . . . . . . . . . . . . . . . 34

3.3 .2 Contrast agents . . . . . . . . . . . . . . . . . 35

3.4 Discussion . . . . . . . . . . . . . . . . . . . . . . . 38

3.5 Conclusion . . . . . . . . . . . . . . . . . . . . . 40

$4 \quad T_{1}$ mapping: what are we measuring? $\quad 43$

4.1 Introduction . . . . . . . . . . . . . . . . . . . . 43

4.2 Theory . . . . . . . . . . . . . . . . . . . . . . . 44

4.3 Materials and Methods . . . . . . . . . . . . . . . . . 46

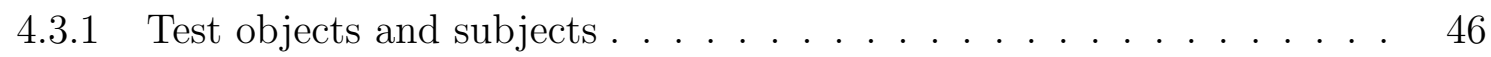

$4.3 .2 \quad$ MR Protocols . . . . . . . . . . . . . . . . . . 47

$4.3 .3 \quad R_{1}$ estimation $\ldots \ldots \ldots \ldots \ldots \ldots \ldots \ldots \ldots$

4.3 .4 Statistical Analysis . . . . . . . . . . . . . . . . . . . 49

4.4 Results . . . . . . . . . . . . . . . . . . . . . . . . . 51

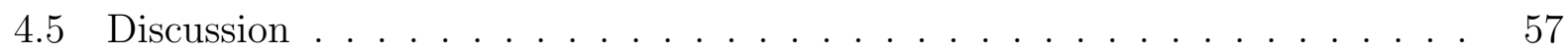

4.6 Conclusion . . . . . . . . . . . . . . . . . . . . . . . . . 59

5 Quantification of edited magnetic resonance spectroscopy: a comparative $\begin{array}{ll}\text { phantom based study of analysis methods. } & 61\end{array}$

5.1 Introduction . . . . . . . . . . . . . . . . . . . . . . 61

5.2 Theory . . . . . . . . . . . . . . . . . . . . . . . . 63

$5.2 .1 \quad$ Spectral processing techniques . . . . . . . . . . . . . 63

5.2 .2 Quantification of spectra . . . . . . . . . . . . . 67

5.3 Materials and Methods . . . . . . . . . . . . . . . . . . . . . 70

5.3 .1 Phantom preparation . . . . . . . . . . . . . . 70

$5.3 .2 \quad$ Scan protocols . . . . . . . . . . . . . . . . . . 70

5.3 .3 Analysis methods . . . . . . . . . . . . . . . . . 73

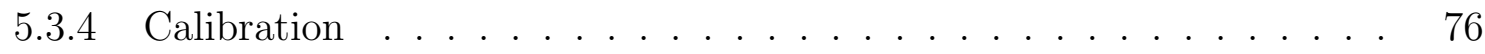

5.4 Results . . . . . . . . . . . . . . . . . . . . . . . . . . 79

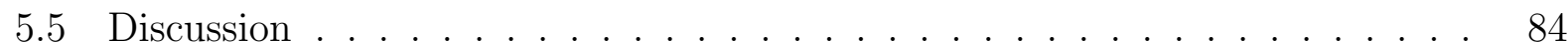


5.6 Conclusion . . . . . . . . . . . . . . . . . . . 86

6 Novel techniques for quantification of metabolites by MRS $\quad 88$

6.1 Introduction . . . . . . . . . . . . . . . . . . . . 88

6.2 Quantum mechanical simulation of MR pulse sequences . . . . . . . . . . 89

6.2.1 Hamiltonian models . . . . . . . . . . . . . . . . . . . 89

6.2 .2 RF fields . . . . . . . . . . . . . . . . . . . 91

6.2 .3 Dissipative simulation f . . . . . . . . . . . . . . . . . . 92

6.3 Optimisation using optimal control . . . . . . . . . . . . . . . . 93

6.3.1 Theory of optimal control . . . . . . . . . . . . . . . 93

6.3.2 Target state selection, objective functions, and constraints . . . . . 95

6.3.3 Robustness considerations . . . . . . . . . . . . . . . . 96

6.3.4 Localisation . . . . . . . . . . . . . . . . . . . . . 97

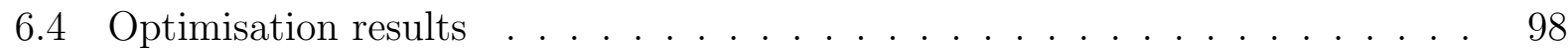

6.4.1 Theoretical evaluation of optimised pulses . . . . . . . . . . . . . 98

6.4.2 Experimental evaluation of optimised pulses . . . . . . . . . . . 102

6.4.3 Experimental $B_{1}$ mapping and calibration . . . . . . . . . . . . 104

6.5 Conclusion . . . . . . . . . . . . . . . . . . 107

$\begin{array}{llr}7 & \text { Conclusion } & 109\end{array}$

$\begin{array}{ll}\text { Appendices } & 113\end{array}$

A Supplementary material for test object analysis $\quad 114$

$\begin{array}{ll}\text { B Supplementary material for relaxometry } & 119\end{array}$

C Tables of chemical shifts and coupling constants for various metabolites. 124

$\begin{array}{lr}\text { D FID-A simulated results. } & 128\end{array}$

$\begin{array}{ll}\text { References } & 138\end{array}$ 


\section{List of Figures}

2.1 Bloch sphere - Excitation . . . . . . . . . . . . . . . . 7

2.2 Relaxation curve plot . . . . . . . . . . . . . . . . . . . 8

2.3 Slice-select gradient diagram . . . . . . . . . . . . . . . . . . . 11

2.4 K-space representation . . . . . . . . . . . . . . . . . . . . 13

2.5 Spin echo and gradient echo pulse sequence diagrams. . . . . . . . . . . . 14

2.7 FID pulse sequence diagram. . . . . . . . . . . . . . . . . . . 23

2.8 STEAM pulse sequence diagram . . . . . . . . . . . . . . . 23

2.9 PRESS pulse sequence diagram $\ldots \ldots \ldots \ldots \ldots$

2.10 MEGA-PRESS pulse sequence diagram . . . . . . . . . . . . . . 27

3.1 Image of one of the Agar gel phantoms . . . . . . . . . . . . . . 30

3.2 Relaxometry sequence diagram. . . . . . . . . . . . . . . . . . . 31

3.5 Agar relaxivity . . . . . . . . . . . . . . . . . . . . . 35

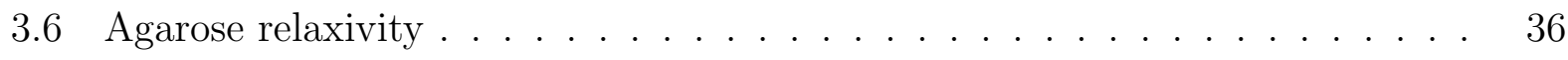

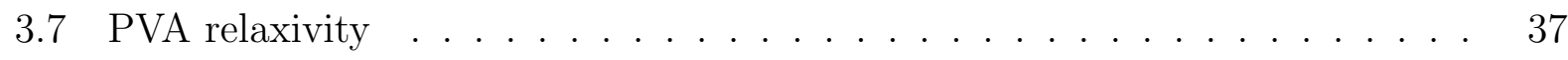

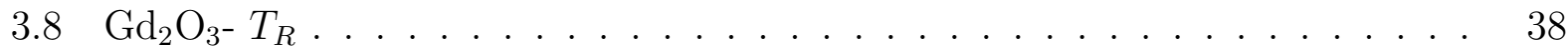

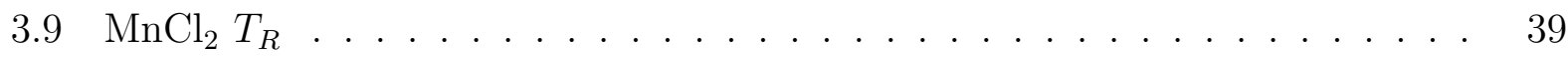

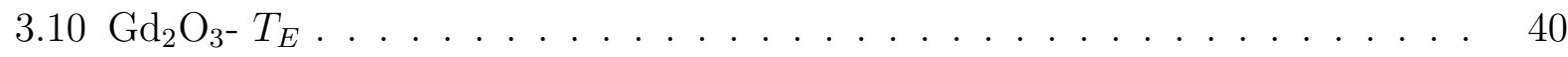

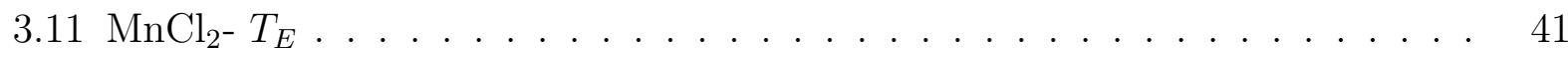

4.1 Inversion recovery pulse sequence diagram. . . . . . . . . . . . . 45

4.2 Brain ROIs . . . . . . . . . . . . . . . . . . . . . . . . . 49

4.3 Grey and white matter delineation $\ldots \ldots \ldots \ldots \ldots$

4.4 Relaxivity curves for $\mathrm{MnCl}_{2}$ solutions and gels . . . . . . . . . . . . 52

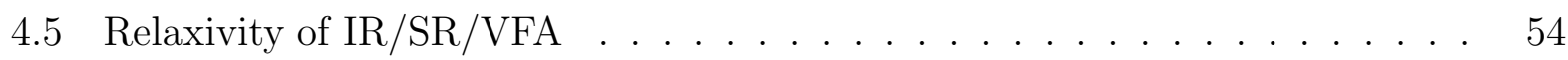

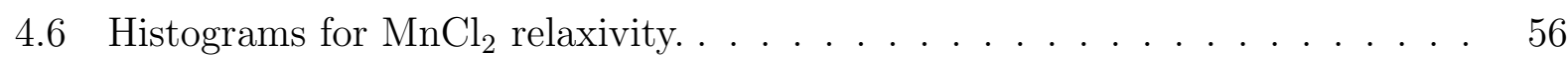

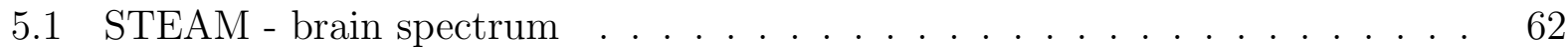

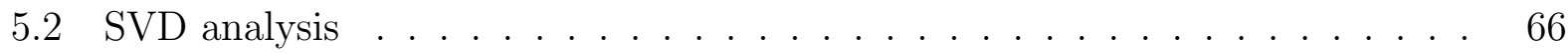

5.3 Images spherical solution and gel phantoms. . . . . . . . . . . 71

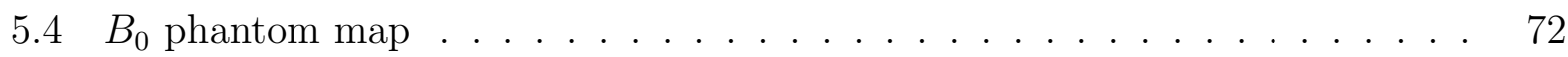


5.5 Temperature dependence of the water peak . . . . . . . . . . . . 77

5.6 NAA spectroscopy $\mathrm{pH}$ effect . . . . . . . . . . . . . . . . . 78

5.7 FID-A simulations of relevant metabolites for phantom series. . . . . . . . . 79

5.8 FID-A simulations for the three most commonly employed GABA models. $\quad 80$

5.9 Simulated MEGA-PRESS ratios $1 \ldots \ldots \ldots$. . . . . . . . . . 80

5.10 Simulated MEGA-PRESS ratios $1 \ldots \ldots \ldots$. . . . . . . . . 80

5.11 Measured GABA to NAA ratio vs known ground truth for the series GABA1. 81

5.12 Measured GABA to NAA ratio vs known ground truth - GABA2. . . . . . . 82

5.13 Measured GABA to NAA ratio vs known ground truth - GLX - body temp . 83

5.14 Measured GABA to NAA ratio vs known ground truth - GLX - room temp. 83

6.2 Optimised pulse: "GABA 106" . . . . . . . . . . . . . . . 99

6.3 Optimised pulse: "GABA 147". . . . . . . . . . . . . . . . . . . 99

6.4 Spectra of optimised pulses for creatine . . . . . . . . . . . . 100

6.5 Spectra of optimised pulses for Govindaraju GABA model . . . . . . . . . . 100

6.6 Spectra of optimised pulses for Kaiser GABA model . . . . . . . . . . . . . . 101

6.7 Spectra of optimised pulses for Near GABA model . . . . . . . . . . . . . . 101

6.8 Spectra of optimised pulses for other metabolites . . . . . . . . . . . 102

6.9 Reference voltage calibration for standard and optimised pulses . . . . . . . 103

$6.11 B_{1}$ field maps for oil and GABA phantoms . . . . . . . . . . . . . . 106

6.12 Rectangular pulse . . . . . . . . . . . . . . . . 106

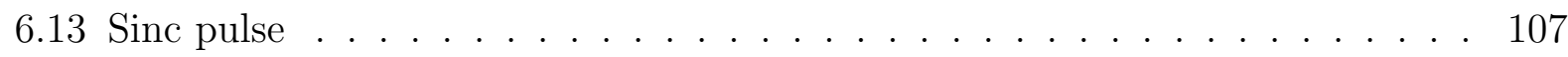

B.1 VFA images . . . . . . . . . . . . . . . . . . . 120

B.2 Inversion recovery and saturation recovery images for $\mathrm{MnCl}_{2}$ solutions. . . . 121

B.3 Relaxation fits if $\mathrm{MnCl}_{2} \ldots \ldots \ldots \ldots \ldots \ldots$. . . . . . . . . . . . . . . . . . .

B.4 Inversion recovery and saturation recovery images for $\mathrm{MnCl}_{2}$ gel phantoms. . 122

B.5 Mean signal vs $T_{I}\left(T_{R}\right)$ graphs for $\mathrm{MnCl}_{2}$ contrast gel phantoms. . . . . . . . 122

B.6 Relaxivity of $\mathrm{MnCl}_{2}$ gel phantoms for different VFA methods . . . . . . . . . 123

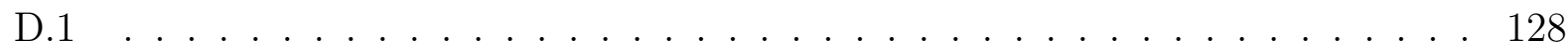

D.1 FID-A GABA: Near . . . . . . . . . . . . . . . . . . . . . . 129

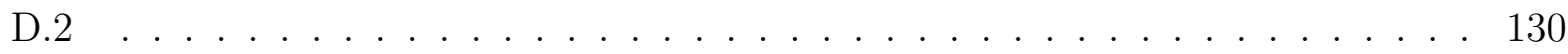


D.2 FID-A GABA: Govindaraju . . . . . . . . . . . . . . . . . 131

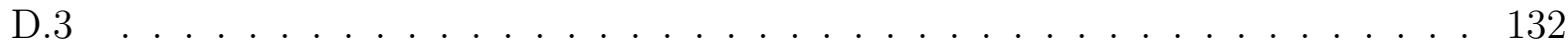

D.3 FID-A Glx: Near . . . . . . . . . . . . . . . . . . . . . . . 133

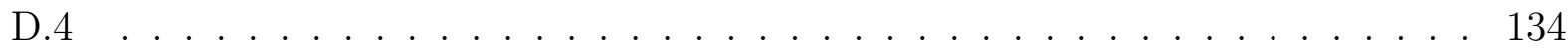

D.4 FID-A Glx: Govindaraju . . . . . . . . . . . . . . . . . 135

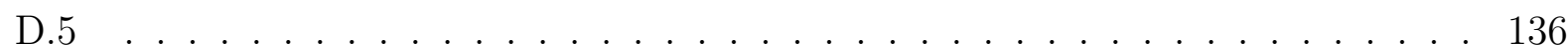

D.5 FID-A Glx: Govindaraju . . . . . . . . . . . . . . 137 


\section{List of Tables}

3.1 Relaxivities of gels and contrast agents . . . . . . . . . . . . . . . . . 42

$4.1 R_{1}$ comparrison of IR and SR . . . . . . . . . . . . . . . 53

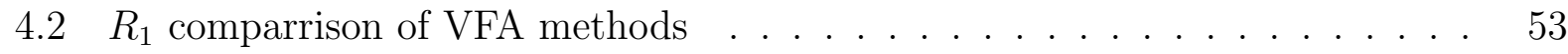

4.3 t-test confidence intervals for IR/SR/VFA . . . . . . . . . . . . . 54

4.4 Relaxivity of $\mathrm{MnCl}_{2}$ for different protocols. . . . . . . . . . . . . . . . . 55

4.5 Mean values of single voxel $R_{1}$ fit parameters for volunteer cohort . . . . . 57

5.1 GABA1 metabolite concentrations . . . . . . . . . . . . . 71

5.2 GABA2 metabolite concentrations . . . . . . . . . . . . . . 71

5.3 GLX metabolite concentrations . . . . . . . . . . . . . . . . . 71

5.4 Linear fit parameters for MRS fitting methods $(1$ of 2$) \ldots \ldots$. . . . . . . 84

5.5 Linear fit parameters for MRS fitting methods $(2$ of 2$) \quad \ldots . . . .85$

A.1 $R_{1} \& R_{2}$ gelling agent . . . . . . . . . . . . . . . . . 114

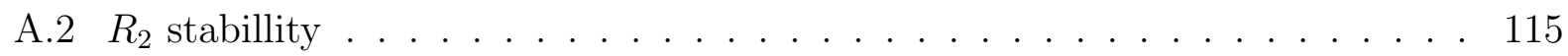

A.3 $R_{1}$ fits for $\mathrm{Gd}_{2} \mathrm{O}_{3}$ scans, with $95 \%$ convidence intervals, and $R^{2}$ values . . . 116

A.4 $R_{1}$ fits for $\mathrm{MnCl}_{2}$ scans, with $95 \%$ convidence intervals, and $R^{2}$ values . . . 117

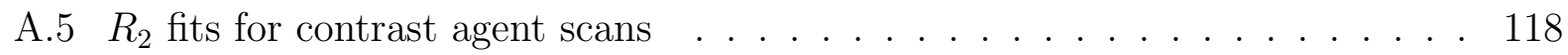

C.1 Chemical shifts and scalar couplings of Creatine . . . . . . . . . . . . 124

C.2 Chemical shifts and scalar couplings of Govindarau GABA model [1] . . . 124

C.3 Chemical shifts and scalar couplings of Kaiser GABA model [2] . . . . . . . 125

C.4 Chemical shifts and scalar couplings of Near GABA model [3] . . . . . . . . 125

C.5 Chemical shifts and scalar couplings of Glutamate [1] . . . . . . . . . . . 125

C.6 Chemical shifts and scalar couplings of Glutamine [1] . . . . . . . . . . . . 126

C.7 Chemical shifts and scalar couplings of Glycine [1] . . . . . . . . . . . 126

C.8 Chemical shifts and scalar couplings of Lactate $[1] \ldots \ldots$. . . . . . . 126

C.9 Chemical shifts and scalar couplings of myo-inositol [1] . . . . . . . . . . . 126

C.10 Chemical shifts and scalar couplings of NAA [1] . . . . . . . . . . . 127 


\section{Abbreviations and definitions}

\begin{tabular}{|c|c|}
\hline$\gamma$ & Gyromagentic ratio \\
\hline$\rho$ & Density matrix \\
\hline$\sigma_{x}, \sigma_{y}, \sigma_{z}$ & Pauli matrices \\
\hline AQSES & Automated Quantitation of Short Echo time MRS spectra \\
\hline$B_{0}$ & Static magnetic field of MRI scanner \\
\hline$B_{1}$ & Time-varying magnetic field used for RF pulses \\
\hline CHESS & chemical shift selective \\
\hline CI & Confidence interval \\
\hline $\mathrm{Cr}$ & Creatine \\
\hline CSI & Chemical shift imaging \\
\hline $\mathrm{CT}$ & Computed tomography \\
\hline DCE & Dynamic contrast enhanced \\
\hline DSS & 2-dimethyl-2-silapentate-5-sulfonate \\
\hline DU & Diazolidinyl urea \\
\hline$f_{0}$ & Larmor precessional frequency \\
\hline FID & Free-induction decay \\
\hline FOV & Field of view \\
\hline GABA & gamma-Aminobutyric acid \\
\hline GANNET & GABA-MRS Analysis Tool \\
\hline $\mathrm{Gd}_{2} \mathrm{O}_{3}$ & Gadolinium oxide \\
\hline Glu & Glutamate \\
\hline Gln & Glutamine \\
\hline Glx & Glutamine + Glutamate \\
\hline
\end{tabular}


$H_{0} \quad$ System/drift Hamiltonian

$H_{n>0} \quad$ Control Hamiltonians

$\hbar \quad$ Planck's reduced constant

IR Inversion recovery

JMRUI Java magnetic resonance user interface

$K_{b} \quad$ Boltzmann's constant

M Net magnetisation

$M_{0} \quad$ Equilibrium magnetisation

MEGA MEscher-GArwood spectral editing scheme

$\mathrm{MnCl}_{2} \quad$ Manganese chloride

MR Magnetic resonance

MRI Magnetic resonance imaging

MRS Magnetic resonance spectroscopy

NAA N-Acetylaspartic acid

NAAG N-acetylaspartylglutamate

NMR Nuclear magnetic resonance

PCr Phosphocreatine

PET Positron emission tomography

PPM parts per million. A field independent frequency scale

PRESS Point RESolved Spectroscopy

PVA polyvinyl alcohol

QA Quality assurance

QUEST Quantitation based on semi-parametric quantum estimation $R^{2} \quad$ coefficient of determination 
$R_{1}$ relaxivity

$r_{2}$

$R_{2}$ relaxivity

$R_{1}$

Longitudinal relaxation rate $\left(R_{1}=1 / T_{1}\right)$

$R_{2}$

Transverse relaxation time $\left(R_{2}=1 / T_{2}\right)$

$\mathrm{RF}$

Radio-frequency

ROI

Region of interest

SAR

Specific absorption rate

SNR

Signal to noise ratio

SR

Saturation recovery

STEAM

STimulated Echo Acquisition Mode

SVD

singular-value decomposition

$T_{1}$

Longitudinal relaxation time

$T_{2}$

Transverse relaxation time

$T_{2}^{*}$

Observed $T_{2}$, compounded by field inhomogeneity

$T_{I}$

Inversion time

$T_{R}$

Repetition time

$T_{E}$

Echo time

$T_{M}$

Mixing time

TARQUIN Totally Automatic Robust Quantitation in NMR

$U$

Unitary propagator

VAPOR variable power RF pulses with optimized relaxation delays

VFA

variable flip angle 


\section{Introduction}

In 1942, Wolfgang Pauli made the suggestion that particles posses an intrinsic angular momentum, a property he called spin. This was followed by the work of Isaac Rabi, Edward Purcell and Felix Bloch in the late 30s and 40s, uncovering nuclear magnetic resonance (NMR) properties resulting from strong magnetic field. It was not until 1970s that MRI truly began to emerge. Two-dimensional imaging methods introduced by Paul Lauterbur [4] were followed by the work of Sir Peter Mansfield [5,6]. By 1980, three dimensional human imaging was possible, and the first commercial MRI scanners began to enter the market. The non-invasive nature of the modality, coupled with excellent soft tissue contrast, has seen MRI continue to grow $[7,8]$, despite competition from imaging modalities such as x-ray, computed tomography $(\mathrm{CT})$, or positron emission tomography (PET), and its relatively high cost. With an estimated three million MRI scans a year in England alone [9], the demand for experienced radiologists to interpret the images has never been greater. The most commonly used MRI techniques generate qualitative data, which can only be effectively interpreted by trained medical practitioners. More quantitative methods could potentially reduce the burden placed on these highly trained staff.

Magnetic resonance offers more than simply structural information. Diffusion weighted imaging provides contrast based on the diffusion of molecules. Knowledge of the directions of diffusion elucidates microscopic information about cell barriers within the body. Perfusion imaging allows mapping of the perfusion of fluids through the body, most commonly used in conjunction with a contrast agent to enhance the sensitivity of the technique. Relaxometry is a technique which aims to estimate fundamental magnetic properties of a particular tissue, known as relaxation rates. While conventional MRI makes use of these properties to generate contrast, relaxometry aims to explicitly quantify them. Magnetic resonance spectroscopy (MRS) provides information about the chemical composition of the tissue in the form of a spectrum. The latter two techniques are the main focus of this thesis.

Quantitative MR relies on the detection of biomarkers; essentially any molecule, structure, or process which is characteristic of a potential medical condition. In the case of relaxometry this means detecting changes in the estimated relaxation rates. Nuclear relaxation 
rates are sensitive to the surrounding chemical environment, and have established ranges for different tissue types. Mapping relaxation rates can offer enhanced contrast over conventional imaging, and unlike conventional imaging, is independent of hardware configuration. In the case of magnetic resonance spectroscopy, detection of biomarkers relies on accurate quantification of the spectrum. MR spectra can provide information regarding the presence of a particular chemical, and also its concentration relative to the others in the same region. In principle MRS can provide metabolic maps, two, or three dimensional spatial distributions of chemical composition (demonstrating it is a very powerful tool). However, analysis of MRS data is difficult. With multiple signals overlapping to varying degrees, decomposing the full signal into its composite parts is a technical challenge.

In order to develop these imaging modalities as quantitative techniques, it is important to select consistent scanning protocols. An MR scan protocol is defined by a set of instructions for the scanner, known as pulse sequences. The "pulses" are separated by timings that can be varied by the operator, producing distinct results. The sequences themselves can also vary, spectroscopy and relaxometry have multiple means of data acquisition. While these methods claim to be measuring the same parameters, there are fundamental differences that inevitably lead to discrepancy in the results. In conventional imaging, these differences are less important, the radiologists simply need to observe contrast in the image. For large scale quantitative imaging to be feasible, the choice of protocol and timings must be optimised and standardised.

In order to explore quantitative imaging, it is important to have a stable reference to compare to, especially for the development of new techniques. While data from human subjects is important, the complexity of the imaging environment, and potential for biological variation make it unfavourable as a reference. Test objects must have stable imaging properties, have a reproducible manufacture procedure, and ideally mimic the conditions of tissue. In MRI, this role can be filled by calibrated solutions or gels. It is possible to adjust the relaxometric properties of a solution through the introduction of contrast and gelling agents, while an MR spectrum may be modulated by introducing the desired chemicals into the solution.

This thesis is focused around development and validation of techniques within these 
quantitative modalities. In the first chapter, an introduction to the fundamental theory of magnetic resonance is presented, including the quantum mechanical basis of MR, and the common techniques employed. The second chapter focuses on the creation, and relaxometric calibration of test objects for MRI, intended for use in quality assurance (QA) of scan data, and development of new techniques. The third chapter continues with relaxometry, using a set of test objects and volunteer scans to evaluate the performance of relaxation rate quantification methods. Chapters four and five focus on techniques in spectroscopy. Chapter four examines the robustness of metabolite quantification, and methods in the analysis of MRS data. Chapter five introduces quantum simulation and control, and how these techniques can be used to develop improved metabolite detection in magnetic resonance spectroscopy. The final chapter collates the results of the previous sections, discussing results in broader terms, and the outlook for future work. 


\section{Theoretical background}

In this chapter, an introduction to the theoretical descriptions of magnetic resonance physics is provided, with further application-specific details dealt with in the respective chapters. The classical analogous description is presented first, followed by the basic quantum mechanical principles. Finally, some specific techniques and terminology are introduced.

\subsection{Basics of magnetic resonance}

An arbitrary rotating body, as described by classical rotational mechanics, has an angular momentum, $\boldsymbol{L}$, due to a rotational velocity, $\boldsymbol{v}$, at a distance $\boldsymbol{r}$ from the centre of rotation. If the rotating body is also electrically charged, the rotational motion creates a current loop, which in turn generates a magnetic field. The magnetic moment, $\boldsymbol{\mu}$, of a body of mass, $m$, is given by:

$$
\boldsymbol{\mu}=\frac{e \boldsymbol{v}}{2 \pi r} \pi r^{2}
$$

This can be rewritten in terms of angular momentum:

$$
\boldsymbol{\mu}=\left(\frac{e}{2 m}\right) \boldsymbol{L}=\gamma \boldsymbol{L}
$$

where $\gamma$ is defined as the gyromagnetic ratio of the object, the ratio of $\mu / L$. If this object is then placed within some externally generated magnetic field, $\boldsymbol{B}_{0}$, its magnetic moment can be written:

$$
\frac{d \boldsymbol{\mu}}{d t}=\gamma \boldsymbol{\mu} \times \boldsymbol{B}_{0}
$$

The amplitude of $\boldsymbol{\mu}$ is constant, so Eq. (2.3) describes the fluctuation of $\boldsymbol{\mu}$ relative to $\boldsymbol{B}_{0}$, or the precession of the magnetic moment. The precession of $\boldsymbol{\mu}$ can also be written in terms of angular velocity, and takes the following form:

$$
\frac{d \boldsymbol{\mu}}{d t}=\boldsymbol{\mu} \times \boldsymbol{\omega}_{0}
$$

Combining Eq. (2.3), and Eq. (2.4) we find:

$$
\begin{aligned}
& \boldsymbol{\omega}_{0}=\gamma \boldsymbol{B}_{0}, \\
& f_{0}=\frac{\gamma}{2 \pi} B_{0} .
\end{aligned}
$$


This is known as the Larmor equation, it describes the relationship between field strength and precessional frequency, $f_{0}$, known as the Larmor frequency. This relationship is a fundamental concept of magnetic resonance physics.

\subsection{Quantum mechanical description}

Classical mechanics is not adequate to fully describe the physics of magnetic resonance. To fully understand this phenomenon, we introduce the concept of spin, an inherently quantum mechanical concept. Unlike its classical equivalent, the magnitude of the angular momentum, $L$, of a body in the quantum regime is limited to a restricted set of distinct values:

$$
L=\hbar \sqrt{I(I+1)},
$$

where $I$ is the angular quantum number, and $\hbar$ is Planck's reduced constant: $\hbar=h / 2 \pi$. Spin can be considered a kind of internal angular momentum of the particle, an intrinsic property like mass or charge. I can only take integer or half-integer values, for bosons and fermions respectively. Most MR applications deal with hydrogen nuclei, i.e., spin 1/2 protons. With the magnitude of $\boldsymbol{L}$ established, the direction must still be defined. This is achieved through the use of a second discrete quantum number, $m$. Consider a particular component of the angular momentum, for example in the $z$ direction:

$$
L_{z}=\hbar m,
$$

where $m$ can take any integer value between $I$ and $-I$, resulting in $2 I+1$ potential values. In the absence of a field, the $2 J+1$ values of $M_{J}$ are degenerate. However, applying a magnetic field breaks this degeneracy, a phenomenon known as the Zeeman effect, or Zeeman splitting. Quantisation of angular momentum will inevitably lead to the quantisation of the magnetic moment. From Eq. (2.2), and Eq. (2.8), we find that the z-component of the magnetic moment is given by:

$$
\mu_{z}=\gamma \hbar m
$$

If we again introduce an external field, $\boldsymbol{B}_{0}$, the classical description of magnetic energy yields the following expression

$$
E=-\mu_{z} B_{0}=-\gamma \hbar m B_{0}
$$


The appearance of the $m$ quantum number here suggests that the magnetic energy of this system is also quantised. For a spin $1 / 2$ particle, such as a hydrogen atom, there are therefore only two energy levels $(m=+1 / 2, m=-1 / 2)$ which have a separation of $\Delta E$ :

$$
\Delta E=\gamma \hbar B_{0}
$$

Transitions between these energy levels can be driven through absorption of a resonant photon, the energy of which can be described by:

$$
\Delta E=h f_{0} .
$$

Equating Eq. (2.12), and Eq. (2.11) we arrive, once again, at the Larmor equation (Eq. (2.6)):

$$
f_{0}=\frac{\gamma}{2 \pi} B_{0}
$$

Hence both classical and quantum formulations are able to arrive at the Larmor equation. The recreation of classical results is a common theme throughout quantum mechanics, and often forms a good sanity check, as the classical description should describe a macroscopic approximation of a quantum phenomena. However, only a quantum description can adequately capture the full dynamics of an MR system.

\subsection{Macroscopic magnetisation}

So far, only individual spins have been considered in the quantum description. However, in a real system we have many spin $1 / 2$ particles, randomly distributed. A spin- $1 / 2$ nucleus has two distinct spin states; $m=+1 / 2$ with $\boldsymbol{\mu}$ parallel with $\boldsymbol{B}_{0}$, known as the $|\uparrow\rangle$ spin state, and $m=-1 / 2$ with $\boldsymbol{\mu}$ anti-parallel with $\boldsymbol{B}_{0}$, known as the $|\downarrow\rangle$ spin state. The difference in population between the high and low energy states is in fact the source of the MR signal. The population ratio can be written using the Boltzmann equation:

$$
\begin{aligned}
\frac{n_{\uparrow}}{n_{\downarrow}} & =e^{\Delta E / k_{B} T}=e^{h f / k_{B} T}, \\
& \approx 1+\frac{h f}{k_{B} T},
\end{aligned}
$$

where $n_{\uparrow}$ and $n_{\downarrow}$ are the populations of the $|\uparrow\rangle$ and $|\downarrow\rangle$ states respectively, $k_{B}$ is the Boltzmann constant, $T$ is the temperature in Kelvin, and Eq. (2.15) is a result of a Taylor series. So, for 


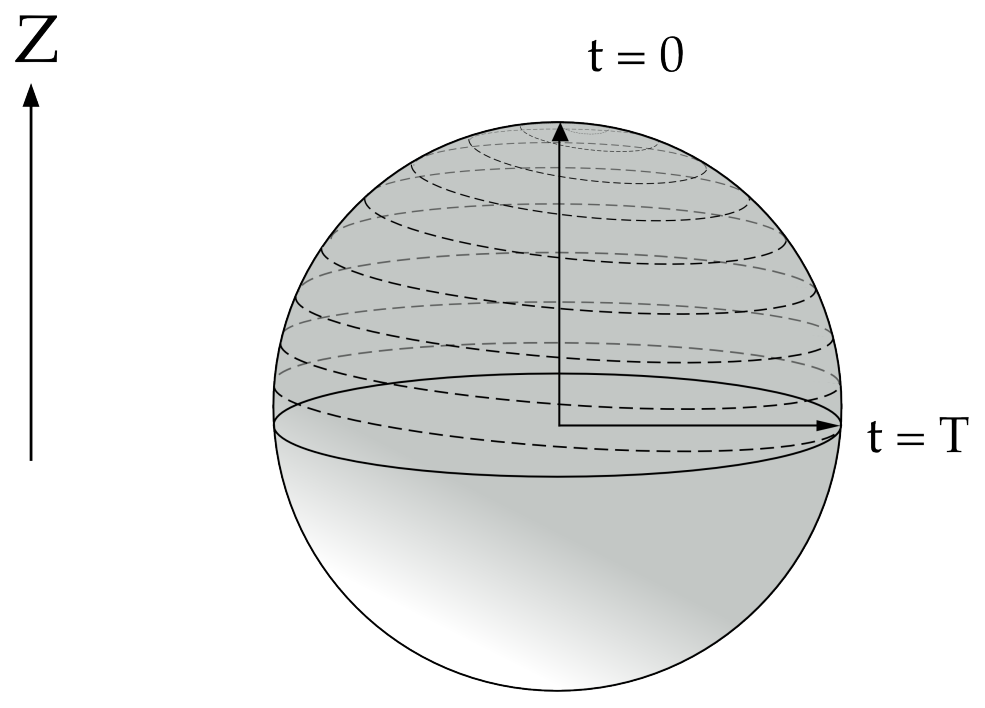

Figure 2.1: The magnetisation is initially in the longitudinal direction at time $t=0$. As the resonant $B_{1}$ field is applied, the magnetisation is rotated into the transverse plane, in a spiral motion, by time $\mathrm{t}=\mathrm{T}$.

a $3 \mathrm{~T}$ scanner, at body temperature, we are attempting to measure a population difference of $0.02 \%$. At thermal equilibrium, the net magnetisation will be parallel to $\boldsymbol{B}_{0}$ in the longitudinal, z-direction, with magnitude $M_{0}$ :

$$
M_{0}=\sum_{k=1}^{N} \mu_{k}=n_{\uparrow} \mu_{z}+n_{\downarrow} \mu_{z}=\frac{\gamma \hbar}{2 \pi}\left(n_{\uparrow}-n_{\downarrow}\right) .
$$

This small additional component to $B_{0}$ would be very difficult to observe experimentally, so any MR experiment involves the rotation of the bulk magnetisation away from the longitudinal direction, towards the transverse plane. This can be achieved by applying an additional time-dependent magnetic field, referred to as $B_{1}$. This second field is oscillatory in nature, and applied perpendicular to $B_{0}$, in the transverse plane. $B_{1}$ can be described according to $B_{1}^{\max } \cos (\omega t)$, where $B_{1}^{\max }$ is the amplitude of the field, and $\omega$ frequency of oscillation of the field. When $\omega$ coincides with the Larmor frequency of the spin system, the net magnetisation, experiences a torque rotating it in a spiral motion towards the transverse plane. This dynamic is demonstrated in Fig. 2.1. As $\omega$ is found to be in the radio-frequency range for most MR applications, the $B_{1}$ are termed RF-pulses. The angle of rotation of the RF-pulse depends on duration and amplitude which is applied. If a pulse is applied for long enough, 
$B_{1}$ can be rotated into the transverse plane, or even beyond, towards the $-z$ direction. This gives rise to $90^{\circ}$ and $180^{\circ} \mathrm{RF}$-pulses, the basic building blocks of many MR pulse sequences. If we switch off $B_{1}$ as the net magnetisation reaches the transverse plane, it will now only experience the original field, $B_{0}$, and will precess as before. However, now the net magnetisation of the sample is perpendicular to the main field, and we are able to measure the electromagnetic force it induces using the resonant receive coils of the scanner. This is the basis of all MR science, and the origin of the signal we observe.

\subsection{Relaxation}

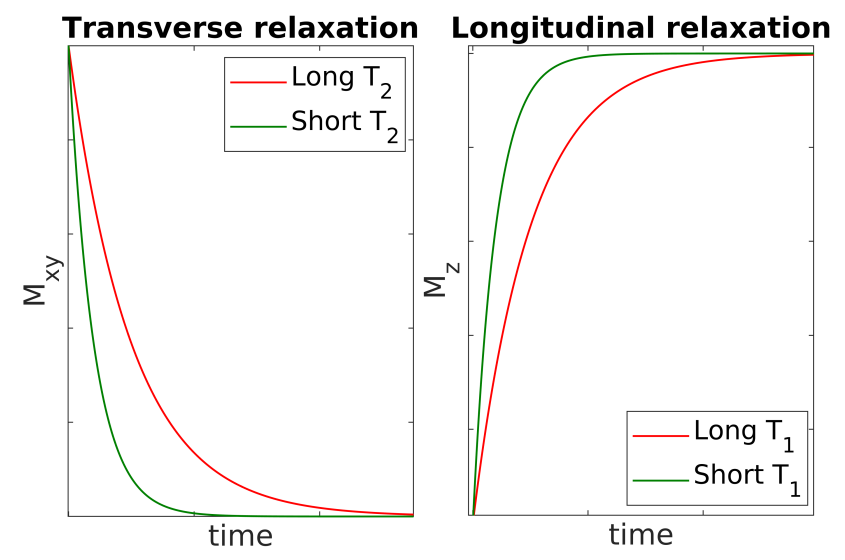

Figure 2.2: Transverse and longitudinal relaxation curves for short (green), and long (red) relaxation times.

Directly after application of a $90^{\circ} \mathrm{RF}$ pulse, the magnetisation, $\boldsymbol{M}$, will begin precessing in the transverse plane. In reality, this motion is a damped precession, and as soon as the pulse has concluded, the magnitude of the transverse magnetisation, $M^{x y}$, will begin to decay. This decay in the observed signal is known as relaxation, and is formed by two distinct processes; longitudinal and transverse relaxation.

\subsubsection{Longitudinal relaxation $-T_{1}$}

Longitudinal relaxation is a decay process which affects the magnetisation in the $z$ direction. If we consider an excited system with $M_{z}(t=0)=0$,i.e., $\boldsymbol{M}$ is precessing in the transverse plane. Over time, this system will begin to lose energy to the surrounding environment 
through heat, and electron interactions, for instance. As this energy dissipates, the spins will increasingly tend to lower energy states, and the system will return towards its thermal equilibrium magnetisation, $M_{0}$. This process is referred to as spin-lattice relaxation, as energy is transferred from the spins to the lattice. This is a general process, not just applicable to lattices, but the name is a throw-back to early NMR experiments. As $\boldsymbol{M}$ relaxes, magnetisation in the $z$ direction is recovered, and takes the following form:

$$
M_{z}=M_{0}\left(1-e^{-t / T_{1}}\right) \text {, }
$$

where, $T_{1}$ is the exponential time constant describing the recovery, and is known as the longitudinal relaxation time. $T_{1}$ can vary greatly from system to system, depending on the molecular structure surrounding the nucleus in question, as well as the strength of $B_{0}$, and temperature of the medium. The differences in $T_{1}$ (and $T_{2}$ ) are of fundamental importance MRI.

\subsubsection{Transverse relaxation $-T_{2}$}

As the name suggests, transverse relaxation is a process that occurs perpendicular to the main static field. At its essence, it is a loss of magnetisation phase coherence of the spin system, resulting in an attenuation of the magnitude of $M_{x y}$. The spins of an excited system will initially have a phase coherence in some transverse direction, say $y$, resulting in a precessing magnetisation $M_{x y}$. Each individual spin in this system is embedded within a complex molecular environment which, due to interactions and shielding, will produce a small perturbation to the main field, $B_{\delta}$. As per Eq. (2.6), the field perturbation also perturbs precessional frequency of the spin:

$$
f_{0}^{\delta}=\frac{\gamma}{2 \pi}\left|\boldsymbol{B}_{0}+\boldsymbol{B}_{\delta}\right|
$$

If the spin system encounters a distribution of fields, $B_{\delta}$, it will in turn exhibit a distribution of precessional frequencies. So, over time, the phase coherence of the magnetisation will be disrupted, as the more quickly precessing spins begin to separate themselves from the slowest. This picture is perhaps a little simplified, in reality the entire ensemble of spins will not be aligned in the $y$ direction, but rather they are thermally distributed about $y$ with a 
net phase coherence transferred from their $M_{0}$ configuration. The decoherence of the spins is an entropic process resulting from thermodynamic interactions between the spins. As such, it can also be referred to as spin-spin relaxation. After a system is excited, spin-spin relaxation will exponentially damp $M_{x y}$ in the following form:

$$
M_{x y}(t)=M_{x y}^{0} e^{-t / T_{2}},
$$

where here, $T_{2}$ is the time constant that governs transverse relaxation. Similarly to longitudinal relaxation, the time constant $T_{2}$ will vary depending on the target substance. $T_{2}$ times for any material will be shorter than the corresponding $T_{1}$. For example, $T_{1}$ of grey matter is on the order of $1400 \mathrm{~ms}$, whilst $T_{2}$ would be around $100 \mathrm{~ms}$ [10]. Experimental quantification, and associated nuances of these time constants will be discussed in greater detail in Sections 3 , and 4 . However, $T_{2}$ as predicted molecular mechanisms, is always found to be longer than the relaxation time observed in practice. The full time, known as $T_{2}^{*}$, is compounded by the effects of field inhomogeneity. Whether a result of some defect in the $B_{0}$ magnet itself, or field distortions resulting from magnetic susceptibility effects, the magnetic field will always have some spatially varying component which increases the transverse relaxation rates.

\subsection{Image formation}

\subsubsection{Localisation}

Applying a $90^{\circ}$ excitation pulse and measuring the decay of the induced transverse magnetization will result in acquiring the signal from the entirety of the sample. This is fine for NMR of homogeneous samples, however, to capture the structure of a more complex environment, for example a person, we must introduce a means to localise the signal. The most common approach to localisation utilises magnetic field gradients. This can be achieved through the introduction if a new field, one that varies linearly with position in $z$ i.e. $B=B_{0}+z G$. As per Eq. (2.6), we therefore also introduce a $z$ dependence of the precessional frequencies of the following form:

$$
f(z)=\frac{\gamma}{2 \pi}\left(B_{0}+z G_{s}\right),
$$

where $G_{s}$ is the gradient of the field. This is the basis for virtually all localised MR pulse sequences. The position dependence of precessional frequencies allows several spatial encod- 


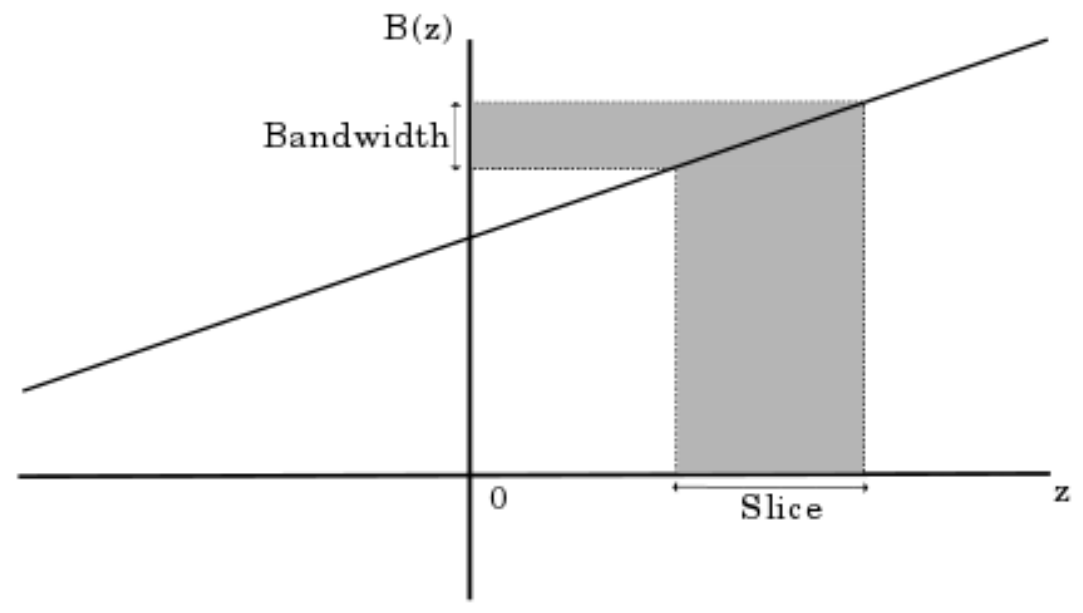

Figure 2.3: Varying the intensity of the field gradient, or the bandwidth of the RF pulse allow a slice of a particular thickness to be selected.

ing methods to be applied. Perhaps the simplest is a practice known as slice selection; a method which involves the simultaneous application of an RF pulse and field gradient to selectively excite a two dimensional "slice" of spins. The bandwidth of the RF pulse can be selected such that it only affects a particular range of precessional frequencies. The range of frequencies, in turn, will correspond to a range of positions in our sample, or the extent of our slice, as shown in fig. 2.3. The slice thickness is defined by the strength of the applied gradient, and the bandwidth of the corresponding pulse, where stronger gradients, and smaller bandwidths create narrower slices. It is common practice, however, for the bandwidth to be held constant, and only the slice-select gradient strength varied. So, we have successfully localised the signal to a two-dimensional slice, however, we need to localise the signal in the other two dimensions in order to acquire a single local volume, or voxel. This requires the introduction of additional gradient localisation methods, frequency and phase encoding.

\subsubsection{Frequency encode gradients}

Another method of localising the MR signal is to apply a gradient during readout, known as frequency encoding. If a linear field gradient is applied, this time in the $x$ direction, we 
introduce an $x$ dependence to our precessional frequencies:

$$
f(x)=\frac{\gamma}{2 \pi}\left(B_{0}+x G_{f}\right)
$$

If the signal is sampled while this field is applied, we are able to relate the signal, as a function of frequency, to the position in $x$ from which it originated.

\subsubsection{Phase encoding gradients}

Suppose we apply a linear gradient in $y$, for time, $t$, at some point between excitation and readout. While the gradient is on, we again introduce a positional dependent precessional frequency:

$$
f(y)=\frac{\gamma}{2 \pi}\left(B_{0}+y G_{y}\right)
$$

When the gradient is then turned off, the entire system precesses at its original frequency, $f_{0}$. While the gradient was active, a position-dependent phase offset was introduced to the system. The magnitude of the phase shift is given by:

$$
\phi(y)=\gamma G(y) t
$$

Combining these localisation techniques for the gradient directions, allows us to localise the signal in all three dimensions. The result is a signal containing both frequency and phase information, localised to a single slice. This frequency and phase information can be related to the spatial positions of the sample via the Fourier transform. However, in order to generate the resolution to form a useful image, multiple acquisitions of this localisation scheme are required. A single signal acquisition will populate one line of k-space, a constructed frequency space, related to the image by Fourier transform. A second acquisition of the signal, with the strength of the phase encoding gradient changed, will add another line to k-space, as represented in Fig. 2.4. Repeated acquisition of different phase encoding gradients will iteratively add resolution to the image. There are alternative methods of sampling k-space, for example, spiral, or radial trajectories. These can be advantageous in situations where fewer phase acquisitions are necessary, to reduce scan time for instance, but will not be detailed here. 


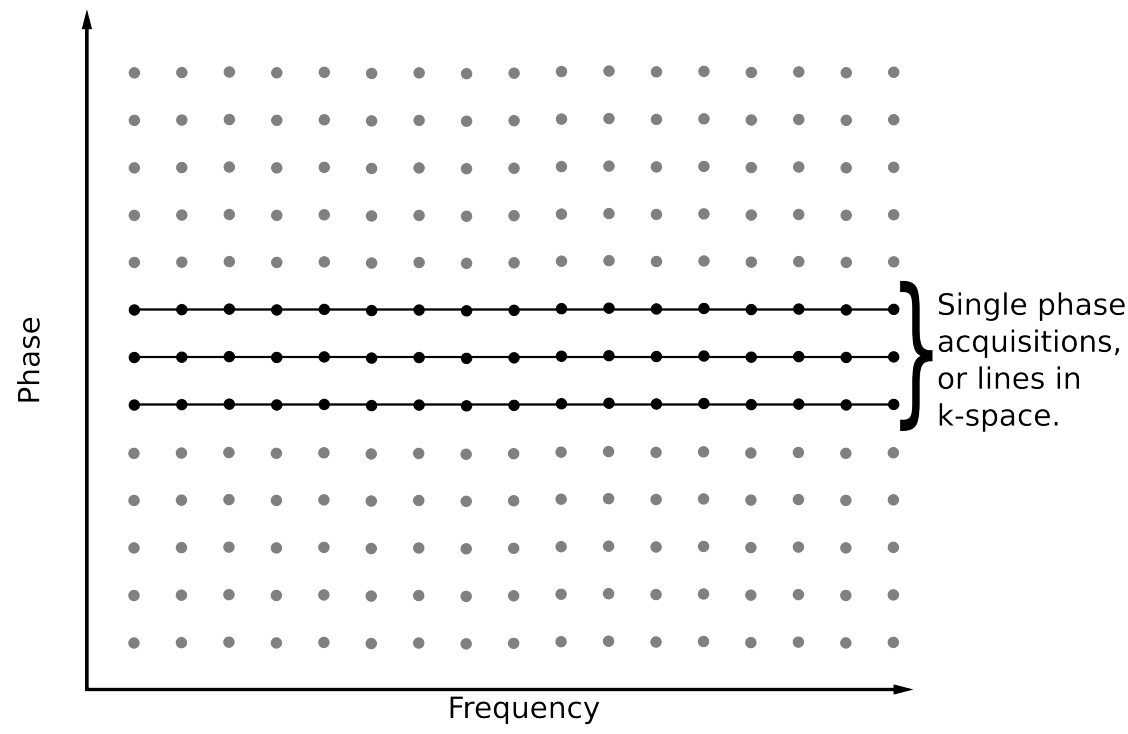

Figure 2.4: Representation of k-space after three acquisitions of a phase encoding gradient. The array of signals (Black dots) are 2D fourier transformed to form an image, the resolution of which will increase with more acquisitions.

\subsubsection{Pulse sequences}

Sequences of gradients and RF pulses can quickly become cumbersome to describe, so are often represented as pulse sequence diagrams. The sequence diagrams usually consist of four parallel axes, each representing a different component of the sequence. An example can be seen in Fig. 2.5. The top line represents the RF-pulses applied, the number and spacing of these pulses will dictate the type of echo we receive. The following three axes correspond to the field gradients in the three orthogonal directions. From top to bottom they are; $G_{z}$ or $G_{\text {Slice }}, G_{y}$ or $G_{\text {phase }}$, and $G_{x}$ or $G_{F r e q}$. The fifth, and final line corresponds to the readout timing of the sequence. There are two common timing parameters associated with sequence diagrams, the repetition time, and the echo time, $T_{R}$ and $T_{E}$ respectively. $T_{R}$ parametrises the length of time between repetitions of the sequence, and is defined as the time between the first excitation pulse in each scan. It is often on the order of seconds to accommodate the recovery of magnetisation between scans. As such, the choice or $T_{R}$ is closely related to the $T_{1}$ of the imaged materials. $T_{E}$ parametrises the length of an individual sequence, and is defined as the time between the first excitation pulse, and readout. $T_{E}$ will always be 
shorter than $T_{R}$, by definition, and due to its relation with echo formation, is closely tied to the $T_{2}$ of the sample.

We have seen that if a single excitation pulse is applied, the spins throughout the entire sample will produce a oscillatory signal known as a free induction decay (FID). However, an FID has no localisation scheme, so signal must be acquired a different way for volumetric sequences. Two common pulse sequences are shown in Fig. 2.5; the spin echo and the gradient echo. A gradient echo can be formed using a single excitation pulse, essentially manipulating the FID to reoccur. The FID signal is first dephased by a field gradient, much like in phase encoding, then later the opposite polarity gradient is applied. The gradients are calibrated such that the spins regain the phase coherence that was lost during the first gradient, and a gradient echo is formed at time $T_{E}$.

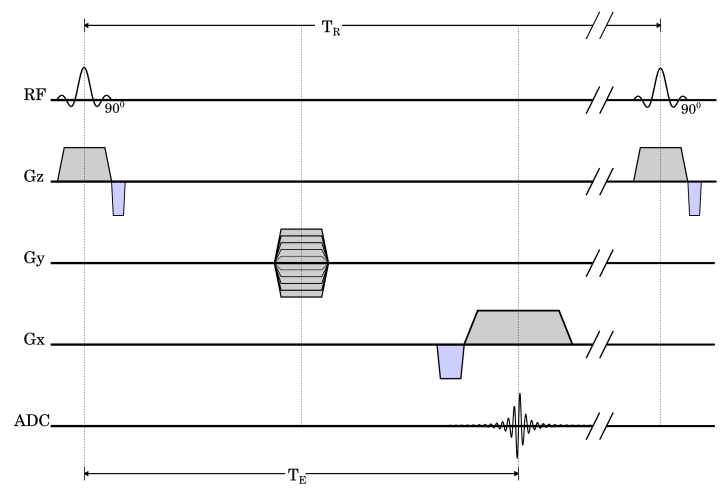

(a) Gradient echo pulse sequence diagram.

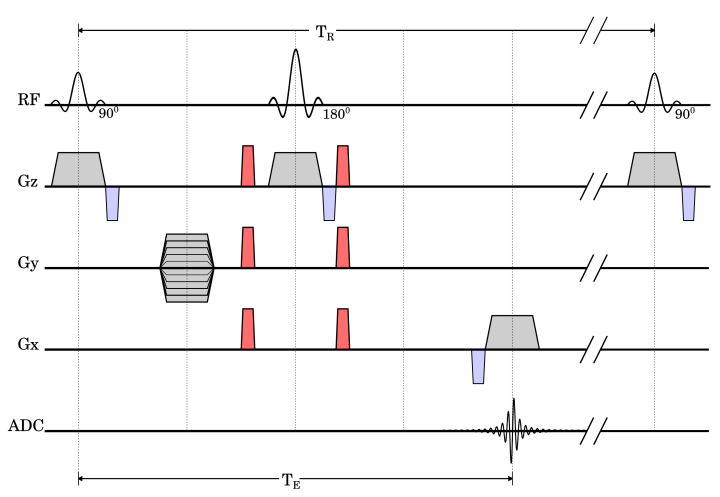

(b) Spin echo pulse sequence diagram.

Figure 2.5: Spin echo, and gradient echo pulse sequence diagrams. Main gradients are shown in grey, re-phase gradients in blue, and crushers in red. Re-phase gradients are calibrated to undo any phase effects resulting from non-phase encoding gradients. The opposite polarity is applied, so the net phase effect is zero. Crusher, or spoiler gradients are used to eliminate unwanted signals. Their intended purpose in (b) is to destroy phase coherence of transverse magnetisation resulting from imperfect $180^{\circ}$ pulses.

The spin echo takes advantage of the symmetry of $T_{2}^{*}$ decay, refocussing the signal into an echo after localisation. The $180^{\circ}$ pulses at time $T_{E} / 2$ reverse the effects of decoherence by flipping them. The slower precessing signals, that were lagging behind the others, now 
find themselves at the front, and vice versa. The result is a spin echo at time $T_{E}$. The signal profile produced by different sequences can quickly become very complex. It is advantageous to model the evolution mathematically, using a set of coupled differential equations, the Bloch equations.

\subsection{The Bloch equations}

In 1946, Felix Bloch described the phenomenon of magnetic induction [11]; the origin of the MR signal. He derived a set of equations that model the evolution of the magnetisation during an MR experiment. Earlier, it was shown that the equilibrium magnetisation, $M_{0}$, can be described as the sum of all individual magnetic moments of the system (2.16). This equation can actually be generalised for total magnetisation [12]:

$$
\frac{d \boldsymbol{M}(t)}{d t}=\boldsymbol{M}(t) \times \gamma \boldsymbol{B}(t)
$$

where $\boldsymbol{B}(t)$ is the generalised field that includes both the static field component, $B_{0}$, and an additional arbitrary time-dependent vector. So, we can influence the magnetisation by introducing a second magnetic field. This has already been discussed briefly, in the form of RF pulses. The magnetic component of an RF field in the $x$ direction can be described as a linearly polarised magnetic field, $\boldsymbol{B}_{1}$ :

$$
\boldsymbol{B}_{1}(t)=2 B_{1}^{\max } \cos (\omega t) \hat{\boldsymbol{x}}
$$

where, $B_{1}^{\max }$ is the amplitude of the field, $\omega$ the transmission frequency, and $\hat{\boldsymbol{x}}$ is a unit vector in the $x$ direction. This field can then be decomposed into two counter-rotating circularly polarised fields:

$$
\boldsymbol{B}_{1}(t)=B_{1}^{\max }[\cos (\omega t) \hat{\boldsymbol{x}}+\sin (\omega t) \hat{\boldsymbol{y}}]+B_{1}^{\max }[\cos (\omega t) \hat{\boldsymbol{x}}-\sin (\omega t) \hat{\boldsymbol{y}}] .
$$

These two components will have different interactions with the spin system. The component co-rotating with the system's magnetisation is considered to have a far more significant effect. While the counter-rotating component does have a small effect [13], it is considered 
negligible in this discussion. In this case, we can write $\boldsymbol{B}_{1}^{x}$ as:

$$
\begin{aligned}
\boldsymbol{B}_{1}^{x}(t) & =B_{1}^{\max }[\cos (\omega t) \hat{\boldsymbol{x}}-\sin (\omega t) \hat{\boldsymbol{y}}] \\
& =B_{1}^{x} \cos (\omega t)+B_{1}^{y} \sin (\omega t)
\end{aligned}
$$

In the absence of relaxation, Eq. (2.24) can decomposed and written in terms of $B_{0}$ and $B_{1}$ :

$$
\begin{aligned}
& \frac{d M_{x}(t)}{d t}=\gamma\left(M_{y}(t) B_{0}-M_{z}(t) B_{1}^{y}\right), \\
& \frac{d M_{y}(t)}{d t}=\gamma\left(M_{z}(t) B_{1}^{x}-M_{y}(t) B_{0}\right), \\
& \frac{d M_{z}(t)}{d t}=\gamma\left(M_{x}(t) B_{1}^{y}-M_{y}(t) B_{1}^{x}\right) .
\end{aligned}
$$

These equations describe the ideal, non-dissipative precession of the field. To include the relaxation mechanisms, we can model them in terms of $T_{1}$ and $T_{2}$ to yield the full Bloch equations:

$$
\begin{aligned}
& \frac{d M_{x}(t)}{d t}=\gamma\left(M_{y}(t) B_{0}-M_{Z}(t) B_{1}^{y}\right)-\frac{M_{x}(t)}{T_{2}}, \\
& \frac{d M_{y}(t)}{d t}=\gamma\left(M_{z}(t) B_{1}^{x}-M_{y}(t) B_{0}\right)-\frac{M_{y}(t)}{T_{2}} \\
& \frac{d M_{z}(t)}{d t}=\gamma\left(M_{x}(t) B_{1}^{y}-M_{y}(t) B_{1}^{x}\right)-\frac{M_{z}(t)-M_{0}}{T_{1}} .
\end{aligned}
$$

The discussion so far dealt with the dynamics of MR in the laboratory reference frame, with a fixed Cartesian coordinate system. However, with precessing magnetisation, rotating fields, and dissipative processes, Cartesian coordinates are not the best choice of frame of reference. A far less cumbersome way to describe these mechanisms is in a co-rotating frame; a Cartesian frame rotating about the static field, with frequency $\omega$. We can then define the components of the magnetisation in this new frame:

$$
\begin{aligned}
& M_{x}^{\prime}=M_{x} \cos (\omega t)+M_{y} \sin (\omega t), \\
& M_{y}^{\prime}=M_{y} \cos (\omega t)-M_{x} \sin (\omega t), \\
& M_{z}^{\prime}=M_{z} .
\end{aligned}
$$


In this new frame, the Bloch equations can be written:

$$
\begin{aligned}
& \frac{d M_{x}^{\prime}(t)}{d t}=-\left(\omega_{0}-\omega\right) M_{y}^{\prime}(t)-\gamma B_{1}^{y^{\prime}} M_{z}^{\prime}(t)-\frac{M_{x}^{\prime}(t)}{T_{2}} \\
& \frac{d M_{y}^{\prime}(t)}{d t}=\left(\omega_{0}-\omega\right) M_{x}^{\prime}(t)+\gamma B_{1}^{x^{\prime}} M_{z}^{\prime}(t)-\frac{M_{y}^{\prime}(t)}{T_{2}} \\
& \frac{d M_{z}^{\prime}(t)}{d t}=\gamma\left[B_{1}^{y^{\prime}} M_{x}^{\prime}(t)-B_{1}^{x^{\prime}} M_{t}^{\prime}(t)\right]-\frac{M_{z}^{\prime}(t)-M_{0}}{T_{1}} .
\end{aligned}
$$

The Bloch equations have many applications, and are routinely used to model MR signal. This approach is utilised in the subsequent two chapters in order to experimentally determine the relaxation times, $T_{1}$, and $T_{2}$, a process known as relaxometry. In order to do this, the Bloch equations must be solved for the sequence used. In the presence of a time-dependent field, the $z$ magnetisation can be defined [10]:

$$
\frac{\delta M_{z}(t)}{d \delta}=\frac{M_{0}+M_{z}(t)}{T_{1}}
$$

where $M_{z}$ is the $z$-component of the magnetisation as it approaches $M_{0}$, the equilibrium value. After application of an RF pulse of flip angle $\theta$ at time $t=0$, the solution is

$$
M_{z}(t)=M_{0}+\left(M_{z}\left(0^{-}\right) \cos \theta-M_{0}\right) e^{-t / T_{1}},
$$

where $M_{z}\left(0^{-}\right)$is the $z$-magnetisation just before the application of the pulse, defined by proton density, hardware callibration, and other factors. If we repeatedly apply the pulse with time period $T_{p}$ then we can enforce the following condition:

$$
M_{z}\left(T_{p}\right)=M_{z}\left(0^{-}\right)
$$

The steady state solution for the transverse magnetisation is then:

$$
M_{x y}=M_{0} \frac{1-e^{-T_{R} / T_{1}}}{1-e^{-T_{R} / T_{1}} \cos \theta} \sin \theta
$$

where $M_{x y}$ is the transverse magnetisation. Eq. (2.44) is the MR signal equation. This equation can be used as a basis for the derivation of all sequences used in the relaxometry studies in subsequent chapters. For example, the transverse magnetisation resulting from a spin echo sequence (Fig. 2.5), can be modelled by setting $\theta$ to $90^{\circ}$. Eq. (2.44) then simplifies to:

$$
M_{x y}=M_{0}\left(1-e^{-T_{R} / T_{1}}\right) e^{-T_{E} / T_{2}} .
$$


A substance with a fixed $T_{1}, T_{2}$, and $M_{0}$ can be modelled as a function of $T_{R}$ and $T_{E}$ across multiple acquisitions, allowing the estimation of $T_{1}$ and $T_{2}$ based on the acquired signal intensities. Conversely, if $T_{1}$ and $T_{2}$ are known, the parameters of the sequence may be optimised to maximise signal intensity, or the contrast between two tissues, for example fat and muscle.

\subsection{Magnetic resonance spectroscopy}

\subsubsection{Chemical shift}

Thus far, the discussion has been restricted to include only a single type of spin, a homogeneous sample of Hydrogen atoms, for instance. While this is a good approximation for clinical application — or indeed water based phantoms - the varying electromagnetic properties of molecules actually provides an interesting avenue of study. The precessional frequency of a spin, given by Eq. (2.6), a product of the gyromagnetic ratio and the magnitude of the magnetic field applied to the spin. However, the electronic environment surrounding the spin in question has an effect. Electrons placed in the external field, $B_{0}$, will begin to precess according to their gyromagnetic ratio, much like the nuclei. This precession will occur in the opposite sense to that of the nuclear spins, and will generate a magnetic moment that opposes $B_{0}$. This results in a reduction in the local magnetic field observed by the nuclei, a phenomenon known as electronic shielding. The Larmor equation for a nucleus can be amended to account for this reduction:

$$
f_{0}=\left(\frac{\gamma}{2 \pi}\right) B_{0}(1-\sigma)
$$

where $\sigma$ is the shielding constant of the atom in question. We find that identical Hydrogen nuclei precess at different frequencies, depending on their electronic environment. This shift is known as the chemical shift of the nuclei. The value of the chemical shift can be trivially expressed in units of $\mathrm{Hz}$, however, as we can see from Eq. (2.46), the shift is dependent on the magnitude of the field, $B_{0}$. This is inconvenient, and would make comparing chemical shifts across different scanners inconvenient. This can be overcome by expressing the shift 
relative to reference compound, $f_{0}^{\text {ref }}$, in units of PPM:

$$
\delta=\frac{f_{0}-f_{0}^{\text {ref }}}{f_{0}^{\text {ref }}} \times 10^{6},
$$

where $\delta$ is the chemical shift in PPM, and $f_{0}$ is the frequency of the sample. The choice of reference compound is a little less straightforward. The obvious choice might be to reference everything relative to water, due to its natural abundance in-vivo. However, the water signal is often suppressed in spectroscopy to improve the detection of other signals, and the frequency of the water peak can shift [14] by $-0.01 \mathrm{PPM} /{ }^{\circ} \mathrm{C}$ due to temperature. This has lead to several external references being used; tetramethylsilane (TMS), the most commonly accepted reference in spectroscopy. Others have been used in acqueous solutions; 3(trimethylsilyl) propionate (TSP), and more notably, 2,2-dimethyl-2-silapentate-5-sulfonate (DSS), a temperature and pH stable compound ideal for the task [14]. In-vivo it is common to use NAA as a calibration peak for chemical shift. Its 2 PPM peak is one of the largest resonances in the brain, and is stable with respect to both $\mathrm{pH}$ [15] and temperature [16]. If we perform a Fourier transform on the complex time profile of the decaying magnetisation, we acquire a frequency distribution of the signal components. To summarise, $N$ nuclei with distinct electronic environments will produce $N$ distinct peaks, with separation dependent on Eq. (2.46), shifted relative to the reference frequency.

\subsubsection{Spin-spin coupling}

Nuclei also interact with one and other. This further changes the spectrum, producing splitting of the spectral peaks that must be considered. There are two means by which spins can interact with one and other. Dipolar coupling is an interaction resulting from the magnetic field produced by one nucleus affecting another. However, in mobile, isotropic fluids, no net effect is observed due to molecular tumbling within the substance. For all applications in this thesis, dipolar interactions can be seen as negligible, and dominated by the effects of J-couplings. J-coupling, or scalar coupling, is an interaction transmitted through the bonds of a molecule, via the intermediate electrons, a process sustained even in mobile, isotropic fluids. Consider a single spin- $1 / 2$ nucleus. It will have two possible energy levels $E_{\uparrow}$ and $E_{\downarrow}$, corresponding to the two spin states, $|\uparrow\rangle$ and $|\downarrow\rangle$, respectively. Therefore 
there is only one possible energy transition; the difference between these levels, $\Delta E$, with a corresponding transition frequency, $f_{T}$, given by Bohr's frequency relation:

$$
\Delta E=h f_{T}
$$

where $h$ is Planck's constant. The frequency of this transition defines the location of its peak within the spectrum. To include the effects of J-coupling we must again look at the electrons of the atom. Electrons, like the nuclei considered here, also have two possible spin states, leading to a total of four for the combined system; $|\uparrow \uparrow\rangle,|\uparrow \downarrow\rangle,|\downarrow \uparrow\rangle$, and $|\downarrow \downarrow\rangle$. These states are not equally likely due to the interaction between the nucleus and its electrons, a process known as Fermi contact. In quantum mechanics, the electron cannot be thought of as ball of charge, as in classical mechanics, but rather a probability distribution. The electrons of our atom have a non-zero probability to be detected within the nucleus, giving rise to the so called hyperfine interaction. Fermi contact leads the electron spins to prefer an anti-parallel alignment to the nuclear spins; $|\uparrow \downarrow\rangle,|\downarrow \uparrow\rangle$. This phenomenon is not entirely relevant for an isolated nuclear spin, but now consider a second nucleus, $B$, chemically bonded to the first, $A$. Uncoupled they produce single spectral resonances at $f_{A}$ and $f_{B}$, respectively. However, when they are bonded, this changes. The electronic interactions within the chemical bond are governed by the Pauli exclusion principle, enforcing that the electron spin states be antialigned. This will in turn affect how energetically favourable the states are, creating four different energy transitions Fig. 2.6. Four energy transitions implies four peaks. The two resonances $f_{A}$ and $f_{B}$ are each split into a pair of smaller peaks, known as a doublet. The locations of these peaks is defined by the coupling strength;

$$
\begin{aligned}
& f_{A^{\prime}}=f_{A}+J, \\
& f_{B^{\prime}}=f_{B}+J,
\end{aligned}
$$

where $J$ is the size of the splitting in units of $\mathrm{Hz}$, and a measure of the coupling strength between the two nuclei. $J$ is a measure of the strength of the coupling, and can be affected by the number of bonds between spins, and their orientation. Unlike chemical shifts, $J$ is independent of field strength, however at higher field strengths, the larger chemical shift spacing may allow better resolution of the J-coupling peaks. 


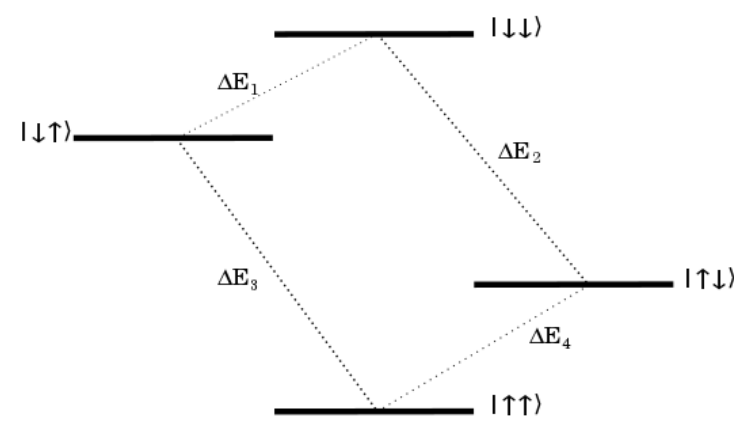

(a) Combined nuclear spin states of a pair of coupled spin-1/2 nuclei.

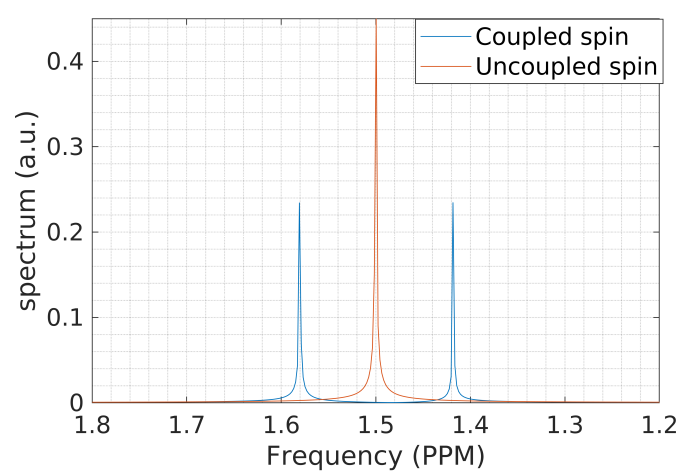

(b) A simulated spectrum of a single resonance, with and without coupling.

Figure 2.6: Uncoupled, these nuclei have one potential energy transition, therefore one resonance per nuclei. In the coupled spin system we see the "splitting" of the energy levels opening 4 possible transitions; $\Delta E_{1}, \Delta E_{2}, \Delta E_{3}$, and $\Delta E_{4}$. This means that each of the resonances splits into a doublet of two peaks.

More generally, a given spin may be bonded to many other nuclei. If a spin is coupled to $n$ equivalent spins, its peak is split into an $(n+1)$-fold multiplet. The nomenclature of the split peak structure is as follows:

- Singlet - A single isolated peak

- Doublet - Two peaks of equal intensity

- Triplet - Three peaks with a 1:2:1 ratio of intensities

- Quadruplet - Four peaks with a 1:3:3:1 ratio of intensities

- Quintet - Five peaks with a 1:4:6:4:1 ratio of intensities

where the ratios of the peaks can be calculated via binomial theorem, or using Pascal's triangle for reference. However, the discussion so far has assumed the separation of the chemical shifts of the peaks is much larger than the coupling. If this is not the case, this simple binomial model does not hold, and second order coupling effects come into play. In order to discuss these relative chemical shift separations, it is conventional to use an 
alphabetic representation. So for example, two well separated spins can be denoted as an $A X$ spin system, and two spins that resonate close to one and other could be denoted an $A B$ spin system. Nuclei with the same chemical environment, said to be chemically equivalent, can also be represented in this fashion using a sub index; an $A X_{2}$ system consists of two chemically equivalent spins well separated from a third spin. The spectrum produced by an $A X_{2}$ system will depend on the coupling structure between them, with unique couplings, the $A$ resonance will split into triplet, as per the binomial description. However, if the two $X$ spins couple with the $A$ spin with the same magnitude, then the $X$ spins are not just chemically equivalent, they are magnetically equivalent, and the $A$ resonance will only split once. Magnetically equivalent nuclei cause no further splitting to the spectrum. The relative height of coupled multiplet peaks can also vary with time due to a process know as J-evolution. After excitation, the $X$ resonance of an uncoupled $A X$ system will precess at its Larmor frequency, $f_{X}$. If a coupling, $J_{A X}$, is introduced, the observed resonance will split into a doublet; two peaks with frequencies $f_{X} \pm J_{A X}$. Over time, the difference between the two resonance frequencies will open a time-dependent phase difference between the resonances, a feature observable by varying the echo time. This phenomenon becomes increasingly complex for nuclei with more couplings, and is only predicted by full quantum mechqanical simulation (Sec.6).

\subsubsection{Common sequences}

Free induction decay The free induction decay (FID) is the simplest possible spectroscopy sequence. The FID consists of a single $90^{\circ}$ excitation pulse followed, instantaneously, by read-out Fig.2.7. The most notable feature of the sequence is the lack of any localisation mechanism. The FID will excite the entire bulk of the sample, producing a non-localised spectrum. This may well be sufficient for phantoms and other homogeneous samples, but for the more complex structures found in-vivo, the resulting spectrum will likely not yield any beneficial data. The term FID is often used synonymously for the signal generated by any spectroscopy sequence. 


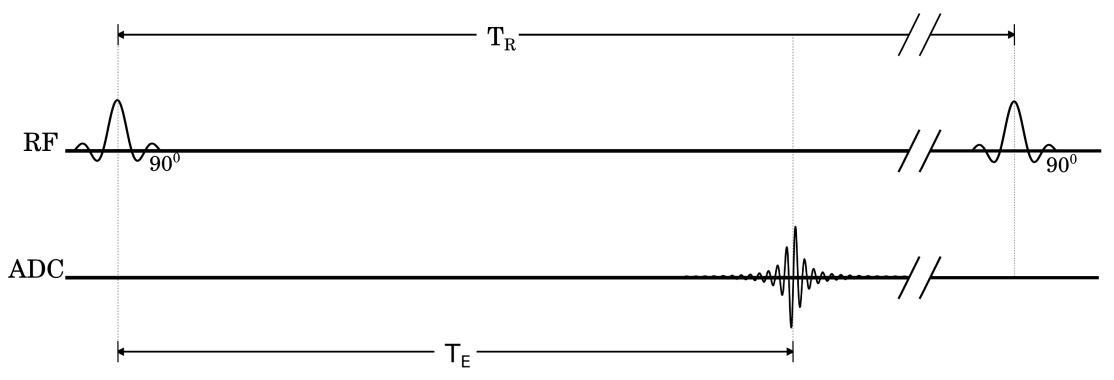

Figure 2.7: FID pulse sequence diagram.

Stimulated echo acquisition mode Unlike the FID, STimulated Echo Acquisition Mode (STEAM) is a single voxel spectroscopy method, utilising a single-shot localisation mechanism. The STEAM sequence consists of three slice selective $90^{\circ}$ Fig. 2.8, localising the spectroscopic signal to a single voxel. The STEAM sequence generates a stimulated echo after a time period $T_{E}+T_{M}$ after the initial excitation pulse, where $T_{M}$ is known as the mixing time. $T_{M}$ is not included in the echo time as the magnetisation is effectively stored in the longitudinal direction, and as such does not undergo $T_{2}$ decay.

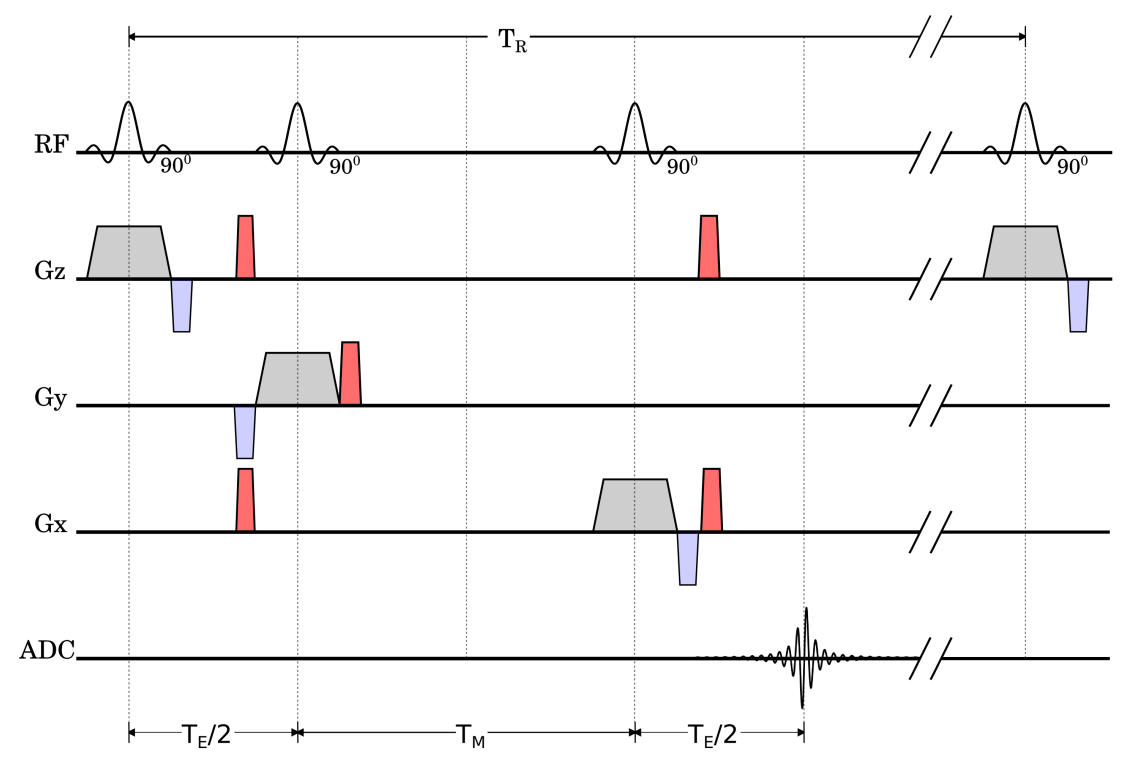

Figure 2.8: STEAM pulse sequence diagram. With main gradients (grey), re-phase gradients (blue), crushers (red). Re-phase and crusher gradients may vary, but this arrangement is typical. 
Point-resolved spectroscopy Point RESolved Spectroscopy (PRESS) is a single voxel spectroscopy technique which has been widely used in 1.5, and 3T MRS. The sequence consists of three slice selective pulses, a $90^{\circ}$, followed by two $180^{\circ} \mathrm{RF}$ pulses, as shown in Fig.2.9 . PRESS produces a spin echo at time $T_{E}$ after the initial excitation pulse, with signal only being emitted by the spins at the intersection of the three slices.

PRESS and STEAM are commonly used techniques, each with their own pros and cons. For example, STEAM is able to achieve a much shorter echo times than PRESS, even as low as $1 \mathrm{~ms}$ [17]. This makes it a preferred technique when attempting to detect metabolites with relatively short $T_{2}$ values. STEAM also has fewer restrictions with respect SAR. Its use of $90^{\circ}$ pulses, rather than $180^{\circ}$, means it deposits less RF-energy in the target medium. However, a major benefit to PRESS is the nature of its echo formation. PRESS produces a spin echo, rather than stimulated echo, meaning it will have an SNR approximately twice that of STEAM for a given $T_{R}$ and $T_{E}$ [18]. This is a huge advantage at 1.5 and $3 \mathrm{~T}$, making it the sequence of choice for the majority of clinical applications, despite the various advantages of STEAM. That being said, at higher field strengths $T_{2}$ values increase, so the shorter echo times of STEAM become more beneficial as we enter the ultra-high field regime.

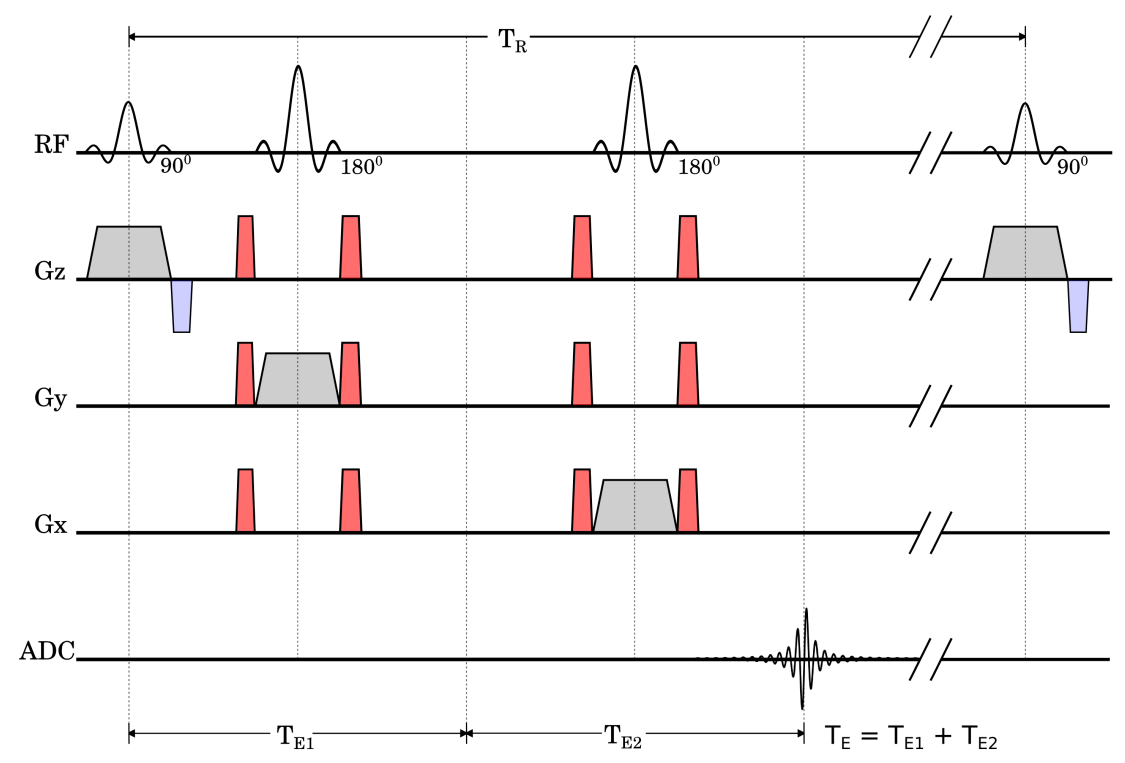

Figure 2.9: PRESS pulse sequence diagram. With main gradients (grey), re-phase gradients (blue), crushers (red). Crusher gradient configuration can vary, but this is a typical arrangement. 
Water suppression Water protons are present at concentrations of approximately 10,000 times that of major brain metabolites, and are therefore the pre-eminent source of the ${ }^{1} \mathrm{H} \mathrm{MR}$ signal. The sequences, as described above, would yield spectra dominated by a water peak, with other metabolic signals ambiguous to the noise floor. In order to detect the peaks of the metabolites, the water signal must be suppressed. The most commonly used way to suppress the water signal is chemical shift selective (CHESS) imaging [19]. CHESS involves applying a frequency selective RF pulse within the range of the water signal, with a specified centre frequency and bandwidth. The pulse rotates the magnetisation of the spins, specifically in this range, towards the transverse plane, where a strong crusher gradient is applied, destroying the phase coherence of the water signal. The frequency selectivity of a pulse is inversely proportional to its length, so the water selective pulses tend to be longer than a standard broadband excitation pulse, typically 10-30ms. CHESS is easily implemented into most spectroscopy sequences, with several CHESS pulses applied before the first excitation pulse of the sequence. Other water suppresion schemes are used, including band selective inversion with gradient dephasing (BASING) [20], variable power RF pulses with optimized relaxation delays (VAPOR) [21], water suppression enhanced through $T_{1}$ effects [22], and water suppression using selective echo dephasing [23], a precursor to the spectral editing technique, MEGA-PRESS, which is the subject of the following section.

Spectral editing In general, editing is the term given to any process which changes the spectral profile resulting from a given experiment. Spectral editing has already been discussed in relation to the suppression of the water signal, but it can be applied in other ways, too. The most commonly used editing sequence is MEGA-PRESS, a scheme first proposed by Mescher and et al. in 1998 [24]. This is a difference editing technique which involves acquiring two data sets - the "edit on" and "edit off", respectively — and subtracting them to acquire the difference spectrum. The edit on scheme uses a PRESS sequence, but adds a pair of frequency selective editing pulses symmetrically placed around the second refocussing pulse Fig. 2.10. The frequency of the pulses is chosen to take advantage of the J-evolution of the target metabolites. The edit off sequence supplies an non-edited spectrum, but for the purpose of symmetry applies the editing at distant frequency, so as not to affect the target 
resonances. Acquisitions are alternated between on and off modes to reduce the influence of hardware limitations, then subtraction is carried out at the end. Although MEGA-PRESS is a relatively general technique, the purpose of the editing pulse is most clearly explained with a specific example. MEGA-PRESS is the most commonly used technique to detect GABA in-vivo [25] [26] [27], and for this purpose, the editing pulse is applied at 1.9 PPM. The pulses will only affect spins which are resonant in this frequency region. In the brain this pertains to the GABA resonances at 1.9 PPM, the NAA peak at 2.0 PPM, the Glx resonances at approximately 2.1 PPM, and other less relevant signals, including the macromolecular peaks in the same region. All of these signals will be removed from the on spectra as a result of the editing, while all other signals are unaffected, and therefore removed during subtraction. Thus far it may appear that the edit on sequence simply removes signal from a specific frequency range, however this neglects the process of J-evolution. While it is indeed only resonances within the edit pulse bandwidth that are affected, some of these spins will be J-coupled to other resonances outside of the edited region, and as the system evolves, these are affected in turn. For GABA, the pair of spins at 1.9 PPM are coupled to a second pair at 3 PPM. Likewise, the Glx peaks at 2.1 PPM have couplings to peaks around 3.75 PPM.

Other notable spectroscopy Sequences Chemical shift imaging (CSI) is a technique that acquires spectra from multiple voxels in a grid. This is achieved by including spatial phase-encoding gradients within another single voxel spectroscopy sequence. This can, in principle, generate metabolic maps allowing spatial the distribution of metabolites to be investigated.

Localisation by Adiabatic SElective Refocussing (LASER) [28] replaces the standard RF pulses with adiabatic ones. While these pulses are longer than their counterparts, they have the advantage of being insensitive to $B_{1}$ inhomogeneity. This is especially useful at ultra-high field strengths, where RF homogeneity becomes more difficult to achieve.

$2 \mathrm{D}$ spectroscopy $[29,30]$ is a technique that acquires multiple spectra across some parameter variation, for example multiple echo times. The length of $T_{E}$ changes the observed spectrum, with short $T_{E}$ favouring short $T_{2}$ spectra, and vice versa. 


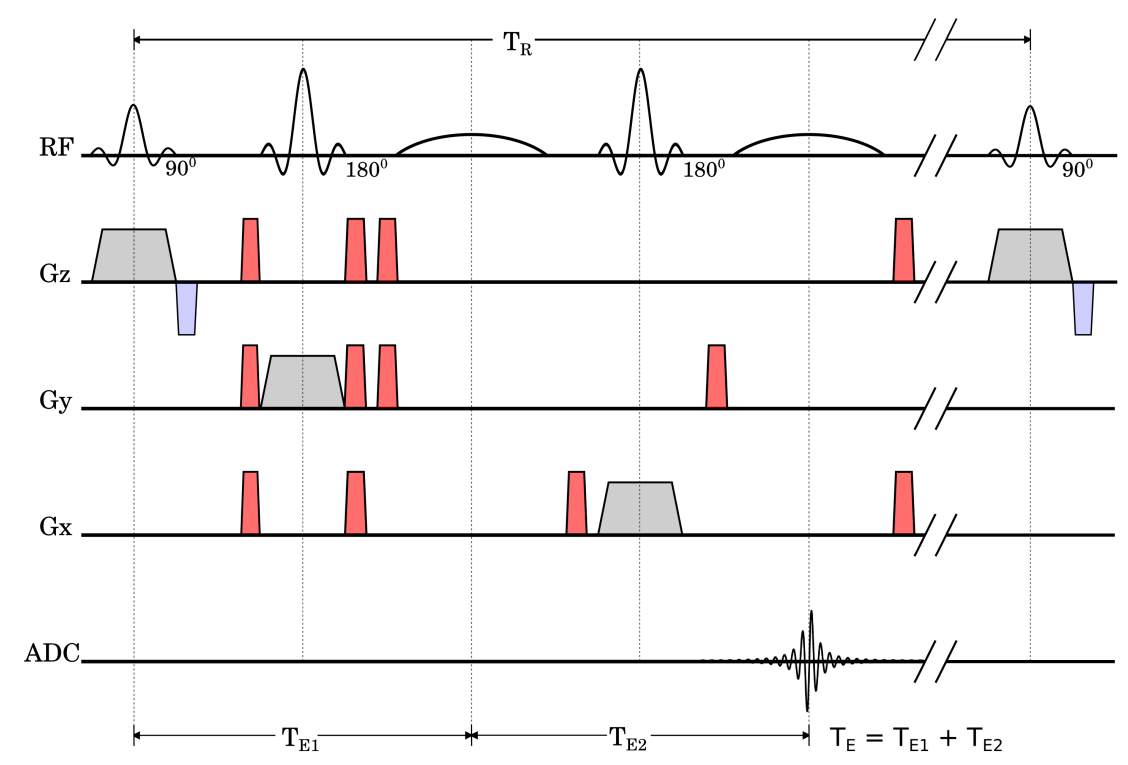

Figure 2.10: MEGA-PRESS pulse sequence diagram, with main gradients (grey), re-phase gradients (blue), crushers (red). The long shallow editing pulses that flank the second $180^{\circ}$ refocussing pulse, are intermittently turned off to acquire the on and off spectra. There is more than one way to implement a MEGA-PRESS sequence; different vendors have their own implementation. [25] 


\section{$3 \quad$ Test objects for quantitative imaging}

\subsection{Introduction}

Test objects with known properties, generally referred to as phantoms in MRI, are an essential prerequisite for quality assurance $[31,32]$ and the development, testing and optimization of new techniques [33-35]. Phantoms can be prepared to mimic in-vivo environments [36], to develop new sequences [37], or enhance existing ones [38,39] as well as protocol development and QA [40-42]. Test objects can also be used to asses field inhomogeneity [31], and even be employed across several imaging modalities, for example, MR-CT [43] and MR-CTultrasound [44].

The type of phantom will differ depending on the requirements of the study, but some aspects are common for all applications. There are some generally desirable criteria for a successful test object: it should have relaxation times comparable to those of human tissue, be robust with respect to manufacturing process and degradation, non-hazardous, and ideally made from inexpensive, readily available materials that anyone can handle [45].

Most MR phantoms fall in one of two categories: aqueous solutions and gel based phantoms. Solutions are typically easier to prepare but generally not "tissue-mimicking " as their native $T_{1}$ and $T_{2}$ times are approximately equal, unlike human tissue, where $T_{2}$ is typically much shorter than $T_{1}$. Gel phantoms are generally prepared using an aqueous solution mixed with a particular gelling agent, the most common being agar and agarose [46-50]. Agarose is a linear polymer, commonly extracted from seaweed, while agar is a composite of agarose and a mixture of other smaller molecules. Alternatives include polyvinyl alcohol (PVA) [51,52], another water-soluble linear polymer, and carrageenan; extracted from a variety of red seaweeds $[53,54]$. The choice and concentration of gelling agent inherently changes the molecular structure, and therefore MR properties, of the phantom. $T_{1}$ and $T_{2}$ can also be affected by paramagnetic contrast agents [55], which can be used to further tune relaxation rates to mimic that of a particular tissue of interest, for example, white matter [50]. The concentration of a given contrast agent has a fixed relationship with the relaxation rate, this is known as its relaxivity, knowledge of which is key in developing phantoms with specific relaxation properties 
In this thesis we will be mainly concerned with phantoms for relaxometry and spectroscopy. Relaxometry is the process of determining the relaxation times $T_{1}, T_{2}$ (and $T_{2}^{*}$ ) of some sample, while spectroscopy exploits differences in chemical shifts and coupling structures of molecules that manifest in a spectroscopic fingerprint, allowing identification and quantification of chemical compounds present in a sample. To facilitate the development of suitable phantoms for these applications, we investigate the relaxivity of common gelling agents (agar, agarose and PVA) and contrast agents (manganese and gadolinium) by characterising a large series of phantoms prepared in-house.

\subsection{Materials and Methods}

\subsubsection{Phantom preparation}

For the investigation of the MRI properties of various gelling agents a set of phantoms made by Gizem Portakal at the Cancer Research Wales Laboratories in Velindre Cancer Centre [42] were used. Three different gelling agents, agar (\#A7002, Sigma-Aldrich, Dorset, UK), agarose (\#A0169, Sigma-Aldrich), and PVA (99+\% hydrolysis degree, \#363146, SigmaAldrich) were tested. For all phantoms Diazolidinyl urea (DU) (\#D5146, Sigma-Aldrich) was added as a preserving agent at a concentration of $6 \mathrm{mg} \mathrm{ml}^{-1}$. The amount of gelling agent was varied, but all were dissolved in $18.2 \mathrm{M} \Omega \mathrm{cm}$ distilled water to the desired level. The solutions were then maintained at a temperature of between $80^{\circ} \mathrm{C}$ and $90{ }^{\circ} \mathrm{C}$ for 30 to $45 \mathrm{~min}$ whilst continuously stirred. The heated solutions were stored in $100 \mathrm{ml}$ high density polyethylene pots for the solidification process, Fig.3.1 . The agar and agarose gels may be left overnight at room temperature to allow solidification to occur, or cooled in a refrigerator or water bath to accelerate this process. The PVA gels required a more complex process of freeze-thaw cycling. The solutions are stored in a freezer at $-20^{\circ} \mathrm{C}$ for $10 \mathrm{~h}$, then removed, and stored at $20^{\circ} \mathrm{C}$ for $14 \mathrm{~h}$. Four freeze thaw cycles were used for these gels. The initial study of gelling agents, contained a total of fifteen phantoms. They consisted of six agar gels at concentrations of $1.0 \%, 1.5 \%, 2.0 \%, 2.5 \%, 3.0 \%$, and $3.5 \%$, six agarose gels at concentrations of $0.5 \%, 1.0 \%, 1.5 \%, 2.0 \%, 2.5 \%$, and $3.0 \%$, and three PVA phantoms at concentrations of $10 \%, 15 \%$, and $20 \%$. Here $2 \%$ refers to $2 \mathrm{~g} / 100 \mathrm{ml}$ of gelling agent. 


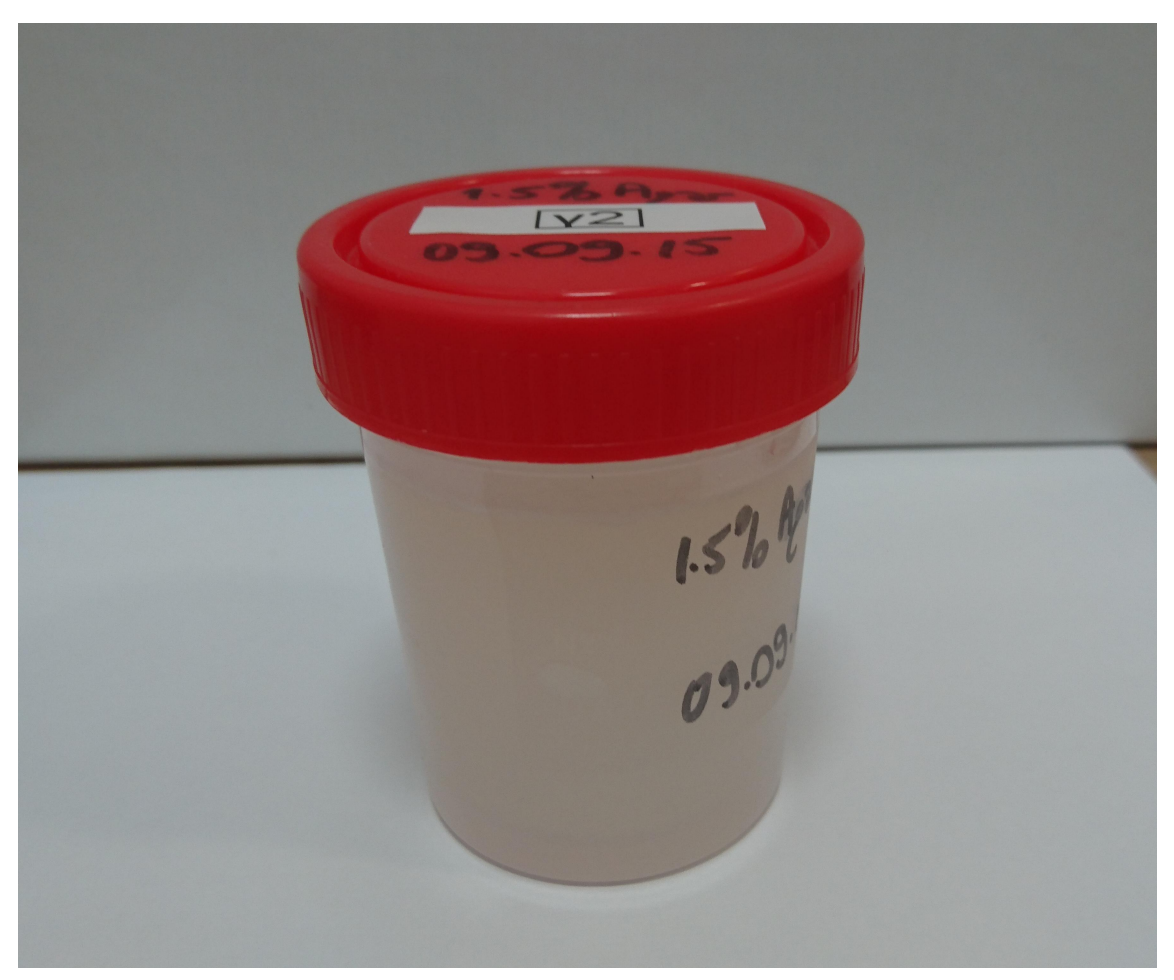

Figure 3.1: Image of one of the Agar gel phantoms

To investigate the relaxation properties for various contrast agents at $3 \mathrm{~T}$, two sets of phantoms were made by myself, at the Centre for NanoHealth, Swansea University. The contrast agents chosen were Manganese chloride $\left(\mathrm{MnCl}_{2}\right)$ and Gadolinium oxide $\left(\mathrm{Gd}_{2} \mathrm{O}_{3}\right)$. For $\mathrm{MnCl}_{2}$, a $0.1 \mathrm{~g} \mathrm{~L}^{-1}$ concentrate solution was made by dissolving manganese (II) chloride tetrahydrate $(98 \%)$ in deionised water. The concentrate was then further diluted to create a series of seven $100 \mathrm{ml}$ solutions with manganese chloride concentrations of $0,10,20,30,40$, 50 , and $60 \mathrm{mg} \mathrm{L}^{-1}$, where $10 \mathrm{mg} \mathrm{l}^{-1}$ corresponds to a $0.0505 \mathrm{mmol}$ concentration of $\mathrm{MnCl}_{2}$. As gadolinium oxide is insoluble in water at neutral $\mathrm{pH}, 4 \mathrm{gL}^{-1}$ citric acid solution was prepared to dissolve the $\mathrm{Gd}_{2} \mathrm{O}_{3}$. $1 \mathrm{~g}$ of di-gadolinium trioxide (anhydrous) was added to form a concentrated solution. The concentrate was then further diluted to create a series of eight $100 \mathrm{ml}$ solutions with with $\mathrm{Gd}_{2} \mathrm{O}_{3}$ concentrations of 1, 2, 5, 10, 20, 30, 40 and 50 $\mathrm{mg} / 100 \mathrm{~mL}$, where $1 \mathrm{mg} / 100 \mathrm{~mL}$ corresponds to a $0.1379 \mathrm{mmol}$ concentration of $\mathrm{Gd}_{2} \mathrm{O}_{3}$. All fifteen solutions were converted to $1 \%$ agar gels using the same process as above, with $0.1 \mathrm{~g}$ of diazolidinyl urea again added as a preserving agent. 


\subsubsection{Scan protocols}

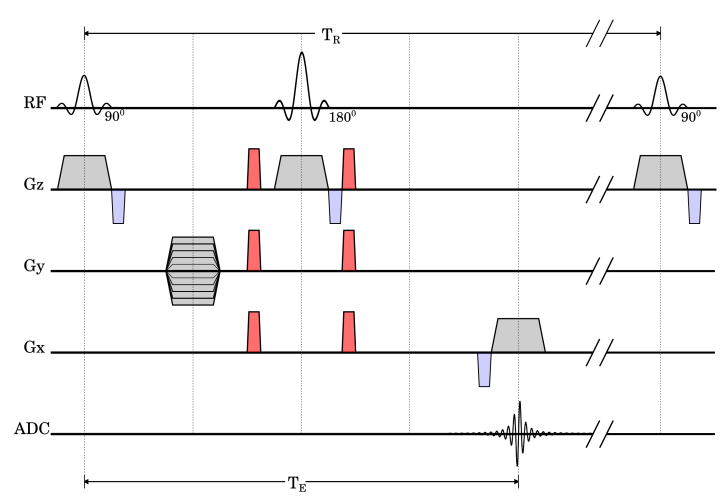

(a) Spin echo sequence diagram used for saturation $T_{1}$ determination.

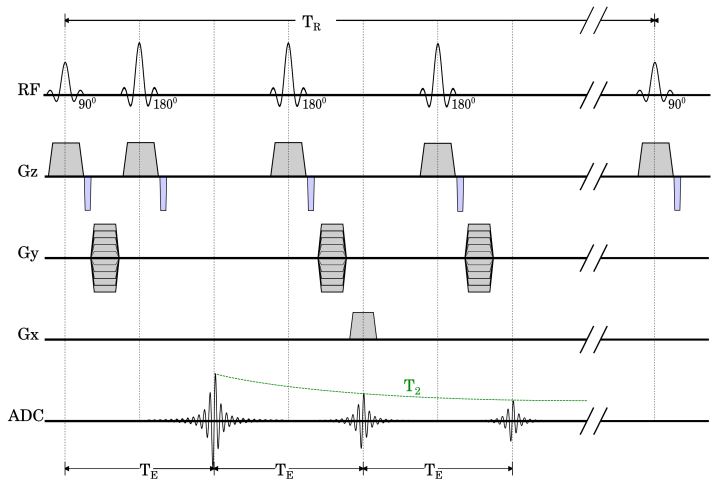

(b) Multiecho sequence diagram. The effect of $T_{2}$ on the measured signal is shown by the green dashed line.

Figure 3.2: Sequence diagrams for $T_{1}$ and $T_{2}$ quantification, respectively. Main gradients (grey), re-phase gradients (blue), and crusher gradients (red).

All scans were conducted at Swansea university's Clinical Imaging Facility using a Siemens 3T Magnetom Skyra. The scanner room is a temperature controlled environment, maintained at $20^{\circ} \mathrm{C} \pm 0.6^{\circ} \mathrm{C}$. The phantoms were placed on the patient table, and the built-in spine coils used for signal acquisition. Specifically, the four channel spine coil element "SP2" was used, as it was assessed to have the highest SNR of all of the arrays. This combination provided the most reproducible set up in terms of phantom arrangement, and coil loading, while minimising the effect of individual channel phase offsets. Longitudinal relaxation was quantified using a saturation recovery spin echo sequence, with a fixed $T_{E}$ of $12 \mathrm{~ms}$, while $T_{R}$ was varied across the following range: 125, 250, 500, 1000, 2000, 3000, 4000, 6000, and 7000 ms. Transverse relaxation estimation can be optimised by using a multi-spin echo sequence. The multiecho sequence allows several signal acquisitions from a single excitation pulse. The magnetisation is repeatedly refocussed creating multiple spin echoes within a single repetition time. The multi spin echo sequence fixed $T_{R}$ at $3000 \mathrm{~ms}$ while $T_{E}$ was varied across 32 unique values: at $15 \mathrm{~ms}$ intervals from 15 to $480 \mathrm{~ms}$. $R_{2}$ protocols were repeated after a year of storage at room temperature to assess long term stability. Both sequence diagrams can 
be found in Fig.3.2.

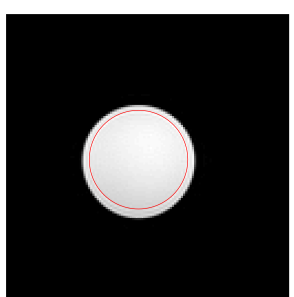

(a) ROI for single phantom

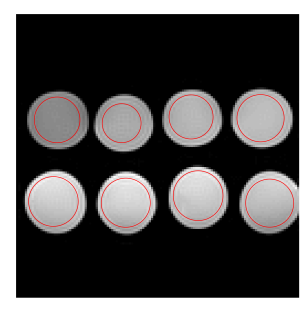

(b) 8 gel arrangement and ROIs

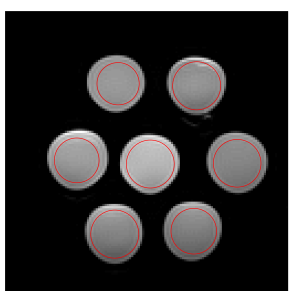

(c) 7 gel arrangement and ROIs

Figure 3.3: Scan arrangements and ROIs (red) for the three set ups used in this study. Arrangement (c) was deemed the optimal, balancing time efficiency and susceptibility effects, and was used for all subsequent studies with multiple phantoms.

For the pure gel measurements, the gels were scanned individually, with the phantom directly on the patient table, directly below the isocentre. The field of view for these scans was $100 \times 100 \mathrm{~mm}$, the matrix size $128 \times 128$, and the readout bandwidth $130 \mathrm{~Hz} /$ pixel. For the contrast agent series, the process was optimised, with the seven/eight gels of the series placed in a symmetric arrangement about isocentre. The field of view was increased to $256 \times 256 \mathrm{~mm}$ to accommodate the arrangement, the matrix size and readout bandwidth were maintained at $128 \times 128 \mathrm{~mm}$, and $130 \mathrm{~Hz} /$ pixel, respectively. A $10 \mathrm{~mm}$ coronal slice was acquired, to provide a cross sectional profile of the phantom(s).

\subsubsection{Analysis}

For each series, a circular region of interest (ROI) was manually defined using one image, such that approximately $10 \%$ of the phantoms extent was excluded (Fig. 3.3), to reduce boundary effects. For the solution series, where each image contained multiple phantoms, a ROI was delineated for each one. The signal was defined as the mean across the circular ROI, and the standard deviation used as a measure of uncertainty. Quantification of the relaxation was achieved by fitting a set of custom fit functions using a trust-region-reflective least-squares minimisation algorithm, using the Matlab curve fitting toolbox. Relaxation rates were estimated, to improve characterisation, with $R_{1}=1 / T_{1}$, and $R_{2}=1 / T_{2}$. 
The starting point for both $R_{1}$ and $R_{2}$ estimation is the MR signal equation, defined in the previous chapter (Eq. (2.45)). For $R_{1}$ quantification, $T_{E}$ was fixed, so the $e^{-T_{E} / T_{2}}$ term is constant, and can be absorbed into a scale factor, $S_{0}$, along with $M_{0}$, and Eq. (2.45) simplifies to:

$$
M_{x y}\left(T_{R}\right)=S_{0}\left(1-e^{-T_{R} R_{1}}\right)
$$

A custom fit function was defined in Matlab, with $M_{x y}$ as the mean signal over the phantom ROI, $T_{R}$ is read in from the protocol information, and $S_{0}$ and $R_{1}$ are parameters to be estimated. A similar reasoning can be applied to the $R_{2}$ determination. If we now fix $T_{R}$, Eq. (2.45) can be simplified by absorbing $\left(1-e^{-T_{R} / T_{1}}\right)$ into a fit coefficient, $S_{0}$ :

$$
M_{x y}\left(T_{E}\right)=S_{0} e^{-T_{E} R_{2}}
$$

or:

$$
\ln \left(M_{x y}\right)=-S_{0}^{\prime} \frac{T_{E}}{R_{2}} .
$$

For $R_{2}$ quantification, the mean signal, $M_{x y}$, was plotted on a logarithmic scale against $T_{E}$, according to Eq. (3.3). A linear fit was applied, with the gradient of the fit providing a measure of $R_{2}$. If $R_{2}$ is high enough, the signal may completely decay over the course of a

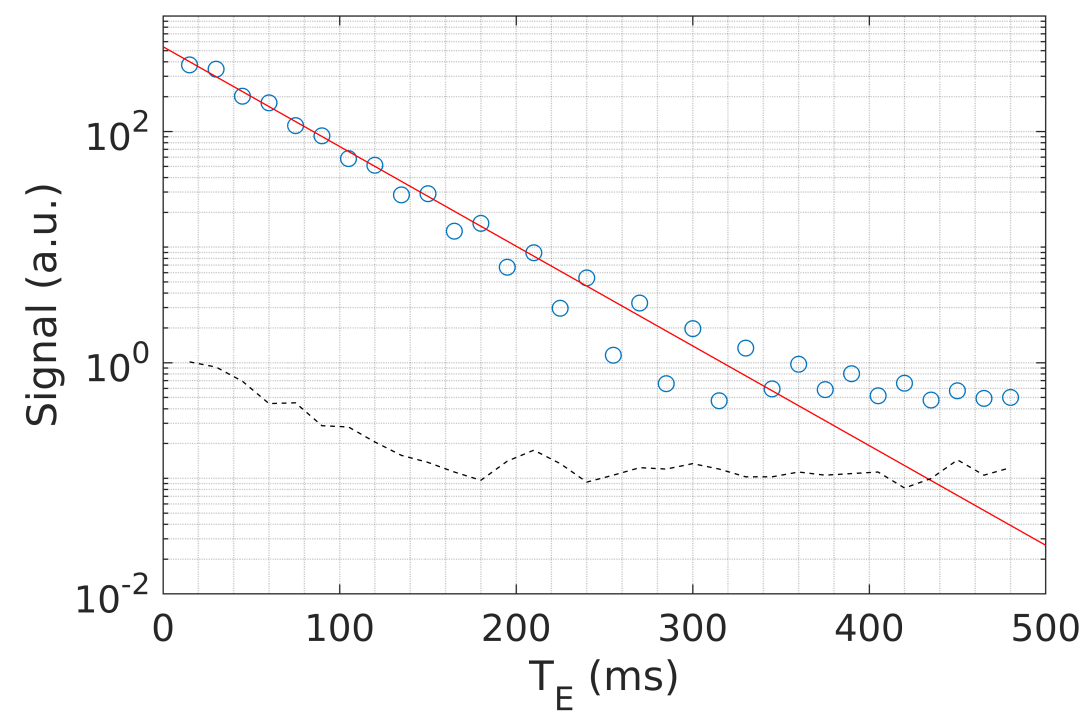

Figure 3.4: Mean signal plot for $T_{E}$ series (blue) and its linear fit (red), with the noise floor estimated using a ROI outside the extent of the phantom (black). 
$T_{E}$ series. This results in the $R_{2}$ trend becoming non-linear for higher echo times, as signal becomes indistinguishable from the noise. To ensure the accuracy of $R_{2}$ estimations, the linear fit must be restricted to avoid fitting the noise floor, so high $T_{E}$ points are excluded from the fits. While this process can be automated, the exclusion threshold, itself, is relatively arbitrary. For this study it was deemed sufficient to manually exclude points in each experiment. Fig. 3.4 shows an example of this process. The quality of each fit is assessed using the coefficient of determination, $R^{2}$, where an $R^{2}$ of 1 indicates a perfect fit, and $95 \%$ confidence intervals (CI) are reported where possible. Relaxivity was estimated by correlating the relaxation rates with the respective concentration of gelling/contrast agent, and applying a linear fit. Again, the trust-region-reflective least-squares minimisation algorithm was used, and the $R^{2}$ and $95 \%$ CI calculated.

\subsection{Results}

\subsubsection{Gelling agents}

$T_{R}$ and $T_{E}$ fits were generally found to be of high quality, with $R^{2}$ not dropping below $96 \%$ across all gels, and all bar 3 fits had $R^{2}$ above 99\%. Fig. 3.5 shows the individual plots for the $T_{R}$ and $T_{E}$ series, as well as the resulting relaxivities for the pure agar gels. With concentrations between $1 \%$ and $3.5 \%, R_{1}$ was found to range between $0.362 \mathrm{~s}^{-1}$ with $95 \%$ CI of $(0.342,0.383)$ and $0.469 \mathrm{~s}^{-1}$ with $95 \%$ of $(0.456,0.482)$, and $R_{2}$ between $5.008 \mathrm{~s}^{-1}(5.098$, $4.918)$, and $12.675 \mathrm{~s}^{-1}(13.059,12.290)$. The $R_{1}$ relaxivity of agar was found to be 0.0354 $(0.0166,0.0543) \mathrm{s}^{-1} \mathrm{mmol}$, and the $R_{2} 2.522(-0.0686,5.113) \mathrm{s}^{-1} \mathrm{mmol}$. For agarose, $R_{1}$ was found to vary between $0.348 \mathrm{~s}^{-1}$ with CI $(0.330,0.367)$ for $0.5 \%$, and $0.399 \mathrm{~s}^{-1}$ with CI $(0.375,0.422)$ for $3.0 \%$ agarose. $R_{2}$ varied between $4.911 \mathrm{~s}^{-1}$ with CI $(4.950,4.872)$, and $21.261 \mathrm{~s}^{-1}$ with CI $(24.158,18.363)$ for the same interval. Agarose was found to have an $r_{1}$ of $0.0222 \mathrm{~s}^{-1} \mathrm{mmol}$ with CI $(0.0159,0.0286)$, and $r_{2}$ of $6.583 \mathrm{~s}^{-1} \mathrm{mmol}$ with CI (6.07, 7.096). Individual plots may be found in Fig. 3.6. Fig. 3.7 shows the results for PVA, which exhibited an $R_{1}$ of $0.690 \mathrm{~s}^{-1}$ with CI of $(0.686,0.694)$ for $10 \%$, and $1.069 \mathrm{~s}^{-1}$ with CI (1.058, $1.081)$ for $20 \%$. Its $R_{2}$ varied between $6.141 \mathrm{~s}^{-1}$ with CI $(6.18,6.10)$ and $13.42 \mathrm{~s}^{-1}(13.83$, 13.01), for $10 \%$ and $20 \%$ respectively. Full $R_{1}$ and $R_{2}$ results for agar, agarose, and PVA 


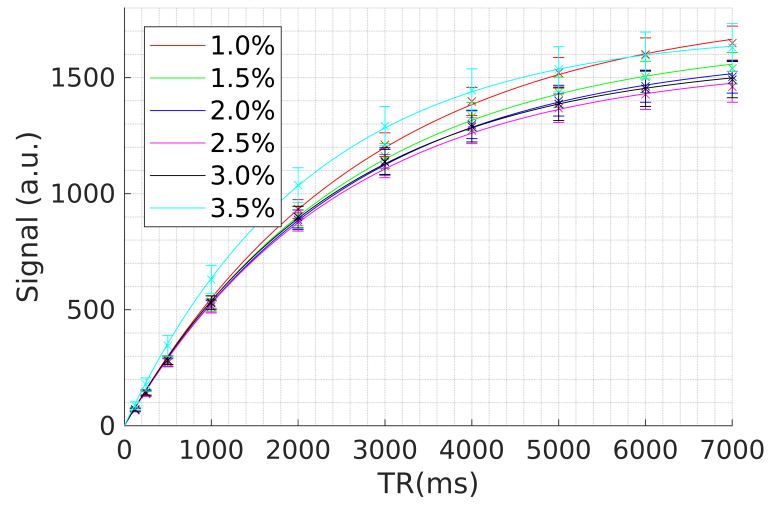

(a) Agar $T_{R}$ fits

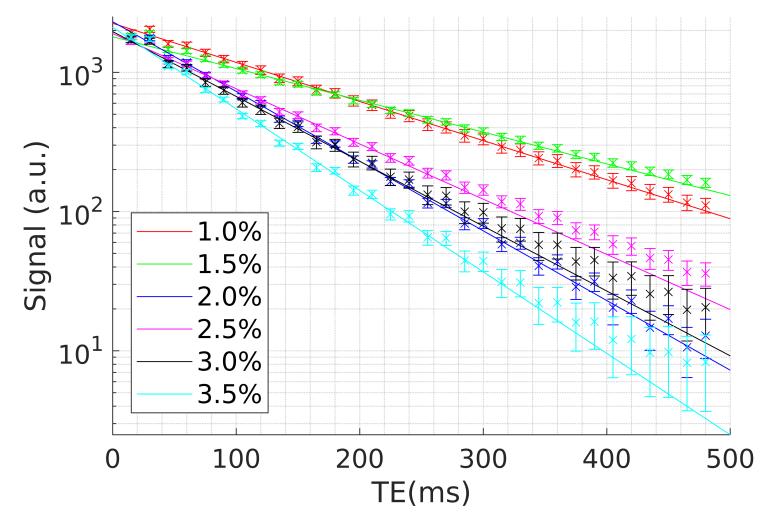

(c) Agar $T_{E}$ fits

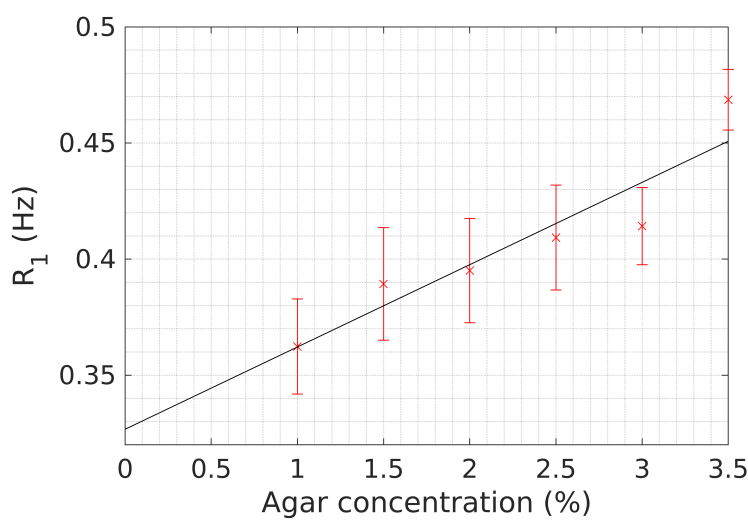

(b) Agar $R_{1}$ relaxivity

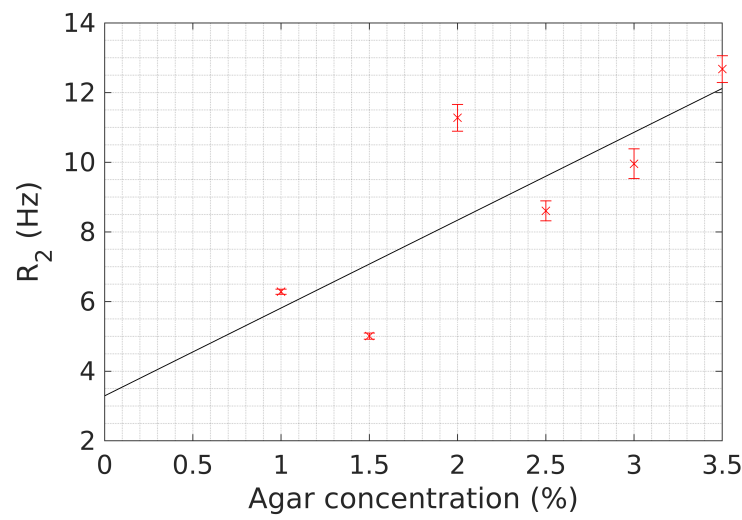

(d) Agar $R_{2}$ relaxivity

Figure 3.5: Agar $T_{R}$ and $T_{E}$ plots, with error bars representing standard deviation across ROI, and resulting relaxivity plot, with error bars representing the $R^{2}$ of the $R_{1}$ and $R_{2}$ fits, respectively.

may be found in Table. A.1, along with $95 \%$ CIs, and $R^{2}$ values.

\subsubsection{Contrast agents}

For the $\mathrm{Gd}_{2} \mathrm{O}_{3}$ the $R_{1}$ protocol was applied on three separate occasions, with $R_{1}$ found to vary between $0.763 \mathrm{~s}^{-1}$ with CI $(0.723,0.802)$ for the $0 \mathrm{mmol}$ phantom, to $30.825 \mathrm{~s}^{-1}$ with CI (29.194, 32.456) for the $0.11 \mathrm{mmol}$ phantom. The $R_{1}$ relaxivity of $\mathrm{Gd}_{2} \mathrm{O}_{3}$ for the three scan sessions was found to be $197.1 \mathrm{~s}^{-1} \mathrm{mmol}(152.7,241.4), 195.1 \mathrm{~s}^{-1} \mathrm{mmol}(149.6,240.7)$, and $177.8 \mathrm{~s}^{-1} \mathrm{mmol}(138,217.6)$, with all values agreeing within $95 \%$ confidence intervals. $\mathrm{Gd}_{2} \mathrm{O}_{3}$ 


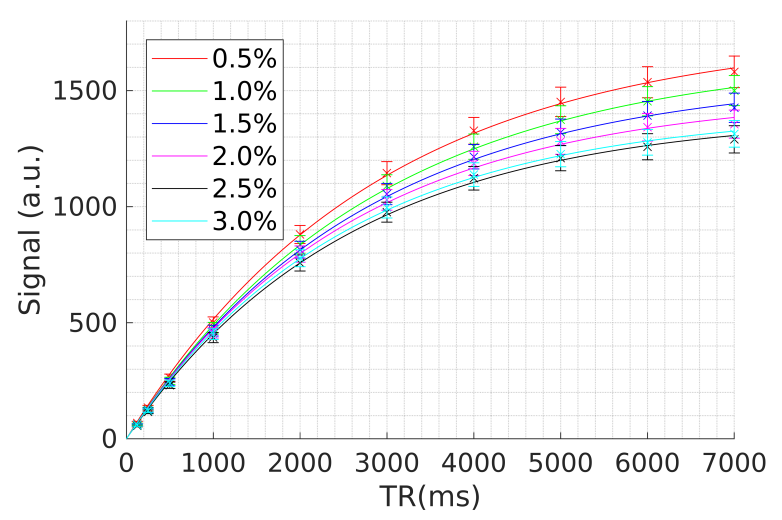

(a) Agarose $T_{R}$ fits

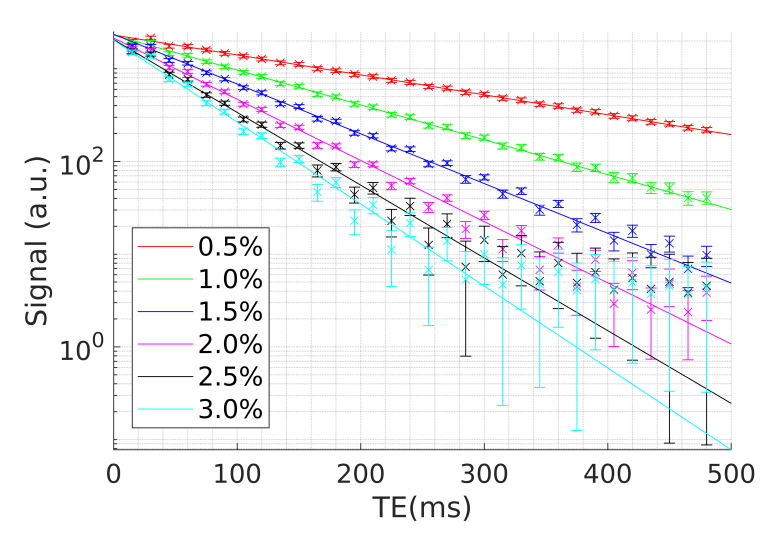

(c) Agarose $T_{E}$ fits

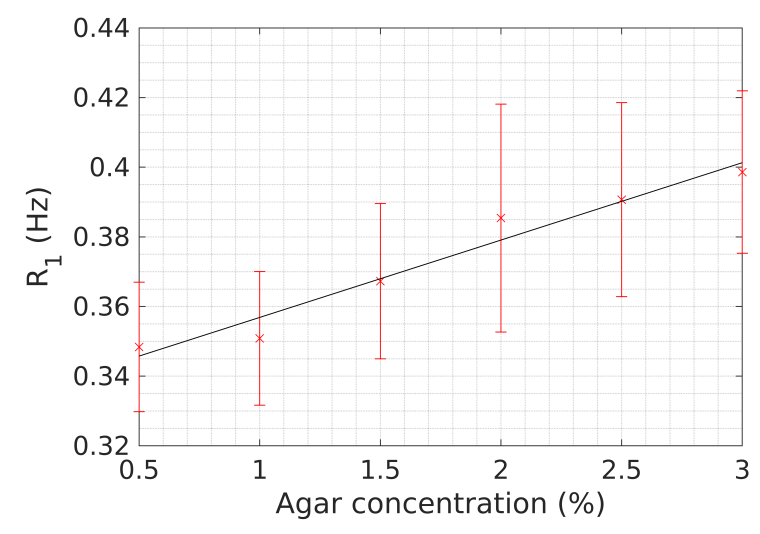

(b) Agarose $R_{1}$ relaxivity

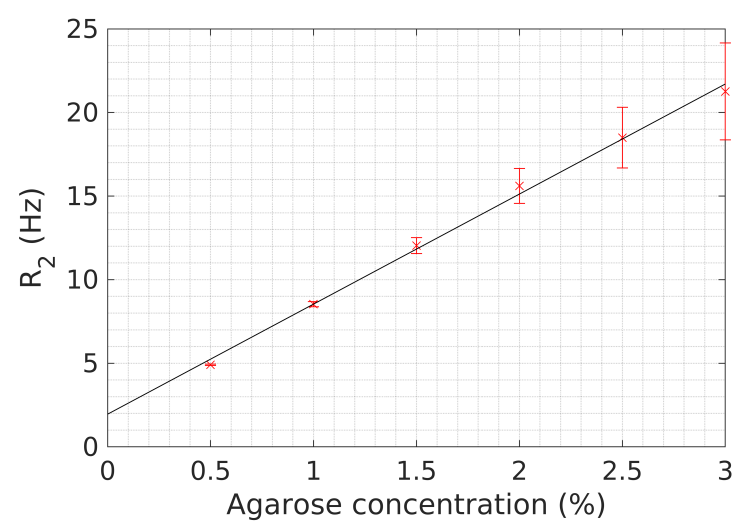

(d) Agarose $R_{2}$ relaxivity

Figure 3.6: Agarose $T_{R}$ and $T_{E}$ plots, with error bars representing standard deviation across ROI, and resulting relaxivity plot, with error bars representing the $R^{2}$ of the individual fit

maintains a consistent $R_{1}$, even after 16 months of shelf storage. Fig. 3.8 shows the $T_{R}$ plots, and resulting relaxivity plots. The $R_{1}$ protocol was also applied multiple times to the $\mathrm{MnCl}_{2}$, Fig. 3.9 shows the $T_{R}$ and relaxivity plots. $R_{1}$ was found to vary between $0.331 \mathrm{~s}^{-1}$ with CI $(0.304,0.358)$, and $4.221 \mathrm{~s}^{-1}$ with CI $(4.154,4.288)$, for the $0 \mathrm{mmol}$ and $0.30 \mathrm{mmol}$ respectively. The relaxivities for the three sessions were $0.631 \mathrm{~s}^{-1} \mathrm{mmol}(0.524,0.737), 0.588$ $\mathrm{s}^{-1} \mathrm{mmol}(0.458,0.719)$, and $0.596 \mathrm{~s}^{-1} \mathrm{mmol}(0.464,0.727)$. Again, the relaxivities are found to be consistent within $95 \%$ CIs, and are stable over a period of 16 months. Tab. A.4 contains the full $R_{1}$ data for the $\mathrm{MnCl}_{2}$ series. For $\mathrm{Gd}_{2} \mathrm{O}_{3}$, the $R_{2}$ protocol was applied only once, with 


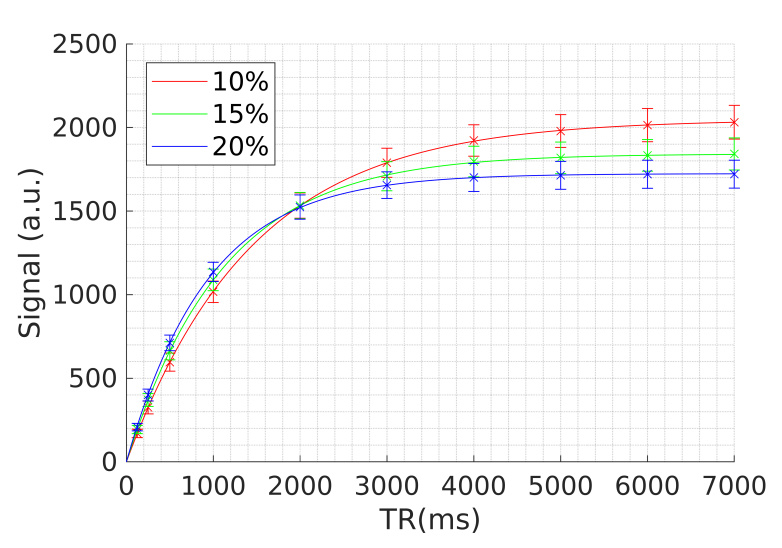

(a) PVA $T_{R}$ fits

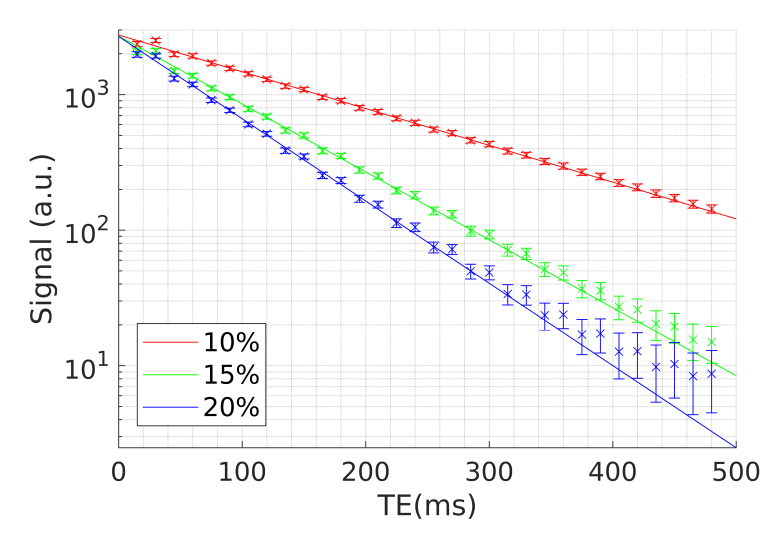

(c) PVA $T_{E}$ fits

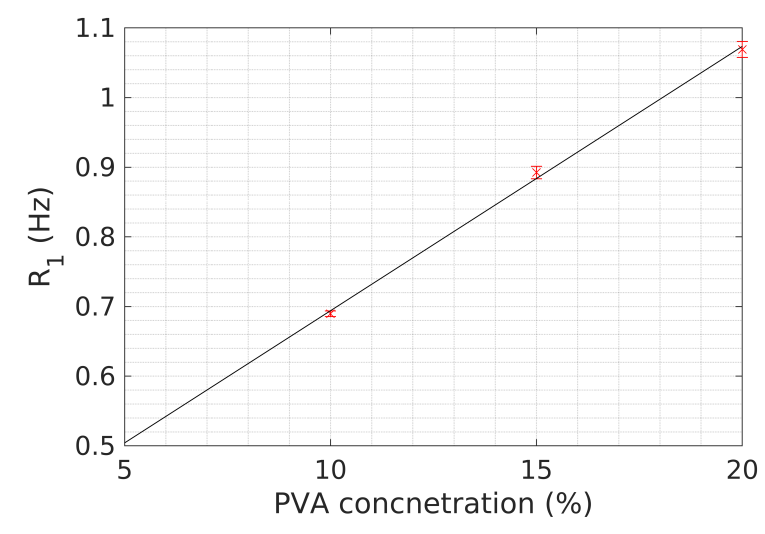

(b) PVA $R_{1}$ relaxivity

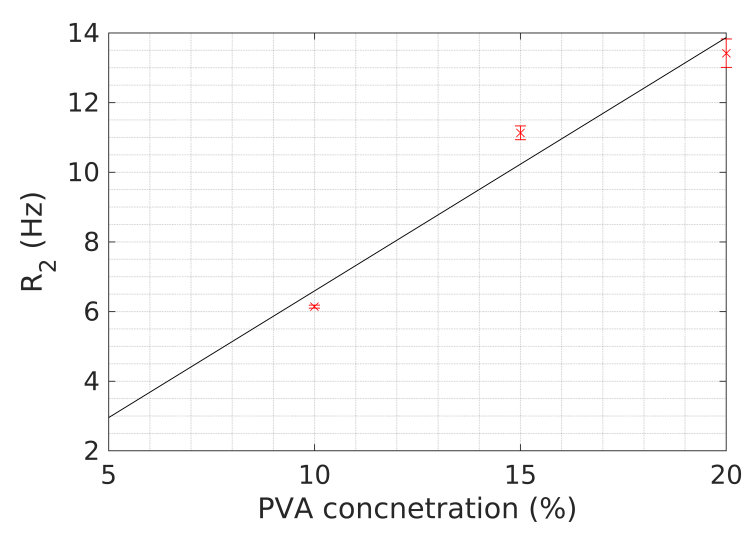

(d) PVA $R_{2}$ relaxivity

Figure 3.7: PVA $T_{R}$ and $T_{E}$ plots, with error bars representing standard deviation across ROI, and resulting relaxivity plot, with error bars representing the $R^{2}$ of the individual fit

$R_{2}$ found to vary between $10.136 \mathrm{~s}^{-1}$ with CI $(10.245,10.027)$ for the 0 mmol phantom, and $47.156 \mathrm{~s}^{-1}$ with CI $(48.430,45.881)$ for the $0.11 \mathrm{mmol}$ phantom. The $R_{2}$ relaxivity of $\mathrm{Gd}_{2} \mathrm{O}_{3}$ was found to be $0.6973 \mathrm{~s}^{-1} \mathrm{mmol}$ with CI $(0.5965,0.798)$. Fig. 3.10 shows the $T_{E}$ fit and $R_{2}$ relaxivity, consult Table. A.5 for full details. The $\mathrm{MnCl}_{2} R_{2}$ protocol was applied on three separate occasions, the quantified $R_{2}$ values were found to vary between $7.061 \mathrm{~s}^{-1}(7.139$, $6.982)$, and $57.390 \mathrm{~s}^{-1}(100.832,13.947)$, for the $0 \mathrm{mmol}$ and $0.30 \mathrm{mmol}$ respectively. The corresponding relaxivities were found to be $102.6 \mathrm{~s}^{-1} \mathrm{mmol}(87.23,117.9), 143.4 \mathrm{~s}^{-1} \mathrm{mmol}$ (116, 170.9), and $152.3 \mathrm{~s}^{-1} \mathrm{mmol}(114.3,190.3)$. The July $2017 \mathrm{MnCl}_{2}$ results display an increase of around $50 \%$ for $r_{2}$ relaxivity, compared to those of the previous year. This 


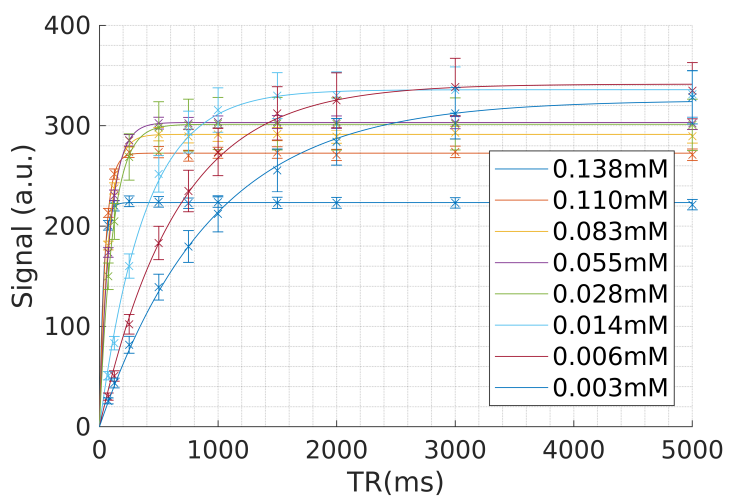

(a) $\mathrm{Gd}_{2} \mathrm{O}_{3}$ Sept 16

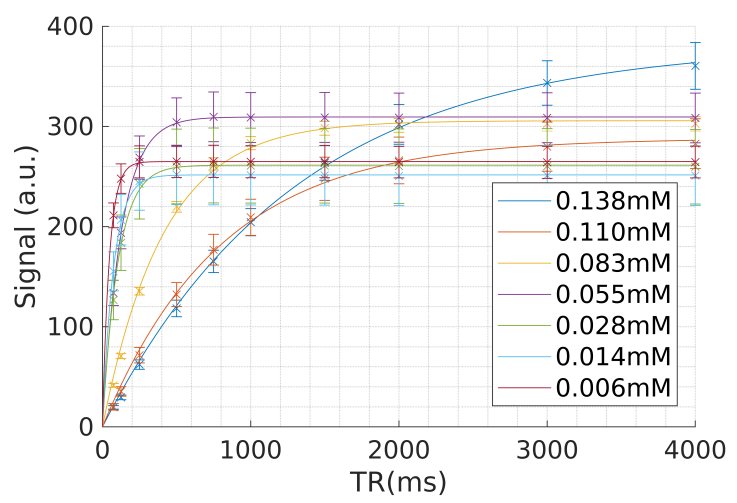

(c) $\mathrm{Gd}_{2} \mathrm{O}_{3}$ Jan 18

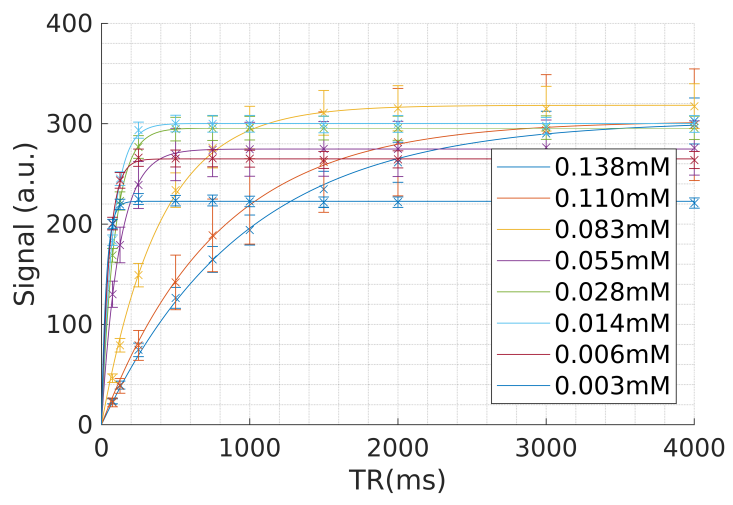

(b) $\mathrm{Gd}_{2} \mathrm{O}_{3}$ Jan 18

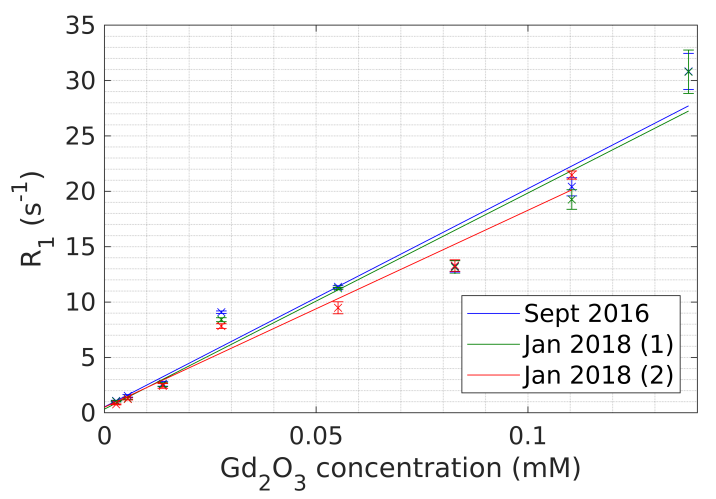

(d) $\mathrm{Gd}_{2} \mathrm{O}_{3} R_{1}$ relaxivity

Figure 3.8: $T_{R}$ plots and resulting relaxivity estimation for $\mathrm{Gd}_{2} \mathrm{O}_{3}$. The multiple $T_{R}$ plots relate to multiple acquistion instances.

feature is not explained by the confidence intervals, and is not reflected in the $r_{1}$ relaxivities. Fig. 3.11 contains the $T_{E}$ fits, and resulting relaxivity, with full details of $T_{E}$ fits included in Table. A.5.

\subsection{Discussion}

Table. 3.1 shows the complete relaxivity results for both the gels and contrast agents. Agar is found to have the lowest $R^{2}$ out of the gelling agents in both $r_{1}$ and $r_{2}$, however, excluding the $3.5 \%$ agar gel increases the $R^{2}$ of the $r_{1}$ fit from 0.87 to 0.92 , suggesting a potential issue with the final concentration. Agarose and PVA are found to have good quality relaxivity fits, 


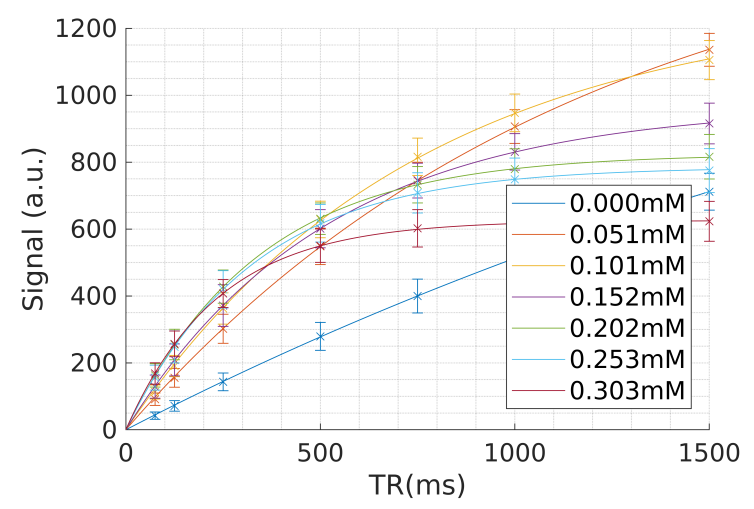

(a) $\mathrm{MnCl}_{2}$ July 16

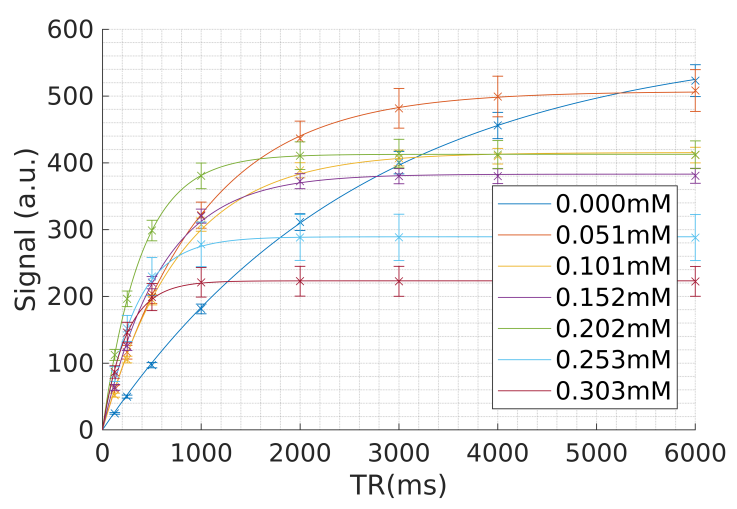

(c) $\mathrm{MnCl}_{2}$ Nov 17

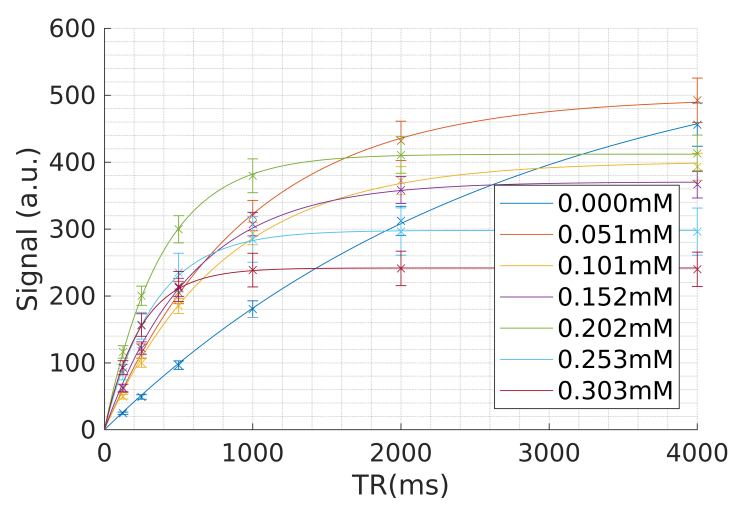

(b) $\mathrm{MnCl}_{2} \mathrm{Nov} 17$

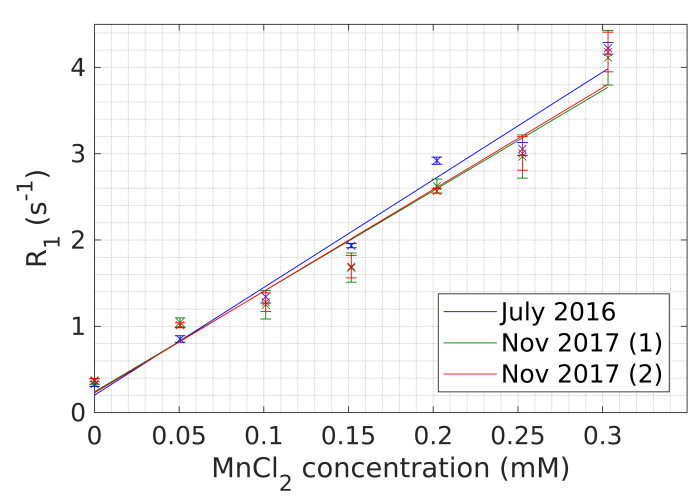

(d) $\mathrm{MnCl}_{2} R_{1}$ relaxivity

Figure 3.9: $T_{R}$ plots and resulting relaxivity estimation for $\mathrm{MnCl}_{2}$. The multiple $T_{R}$ plots relate to multiple acquistion instances.

with $R^{2}$ greater than 0.95 in all cases. Agarose is the strongest $R_{2}$ modifier of the gels, with an almost tenfold increase in relaxivity compared to PVA, whilst maintaining a comparable $r_{1}$. The differences in agar and agarose relaxivities appear to reflect the agar impurities as a primarily $R_{1}$ modifier, with the agarose as the source of agar's $R_{2}$ relaxivity. For the contrast agents, $\mathrm{Gd}_{2} \mathrm{O}_{3}$ produces the strongest relaxivity in both $r_{1}$ and $r_{2}$, and appears to affect both $R_{1}$ and $R_{2}$ in equal measure. Conversely, $\mathrm{MnCl}_{2}$ produces a $r_{2}$ approximately 10 times that of its $r_{1}$. The contrast agent phantoms generally exhibit good long term stability, within $95 \%$ confidence intervals. However, the $2016 \mathrm{MnCl}_{2} r_{2}$ session is a notable exception, exhibiting a $50 \%$ shift in $r_{2}$ compared to the subsequent acquisitions. Surprisingly, a similar effect is not observed in the $r_{1}$ data. So, while the $r_{2}$ increase may be indicative of gel degradation 


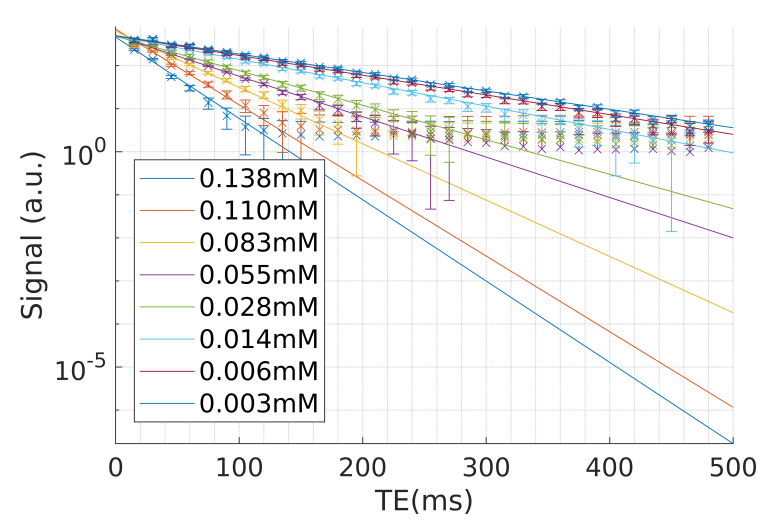

(a) $\mathrm{Gd}_{2} \mathrm{O}_{3} T_{E}$ fit

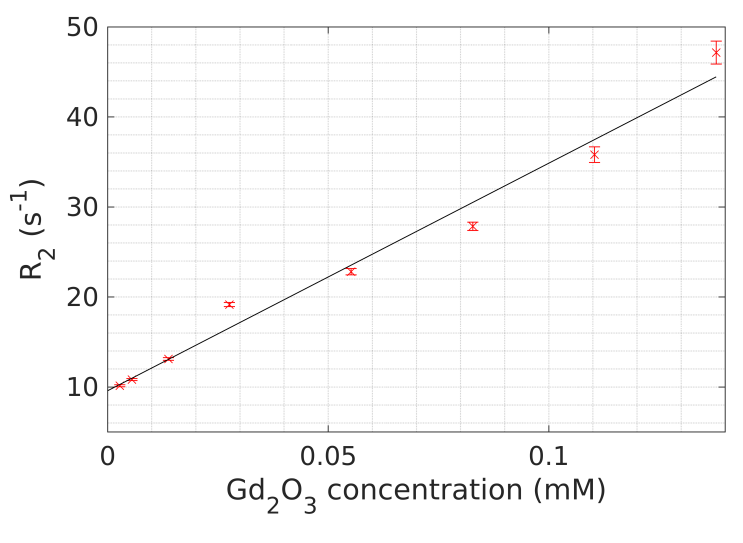

(b) $\mathrm{Gd}_{2} \mathrm{O}_{3} R_{2}$ relaxivity

Figure 3.10: $T_{E}$ plots and resulting relaxivity estimation for $\mathrm{Gd}_{2} \mathrm{O}_{3}$.

following the first session, further study is necessary to elucidate the underlying process. Repeat $r_{2}$ scans of the $\mathrm{Gd}_{2} \mathrm{O}_{3}$ phantoms could aid this endeavour, and will be considered for future work.

Table. A.2 contains the results of the two $R_{2}$ scans, separated by a year. PVA results are omitted from this table due to their visible deterioration. The PVA gels were observed to disintegrate within days of the original scans, and provided a low MR signal when rescanned. The agar and agarose appear visibly unchanged after a year, retaining their original consistency. These results suggest good long term stability of agar and agarose, even when stored at room temperature.

\subsection{Conclusion}

Agarose and PVA produce the best quality relaxivity fits in terms of $R^{2}$, with agarose as a primarily $R_{2}$ modifier. The $R_{1}$ are comparable, but the relatively complex procedure involved in the preparation of PVA will inevitably limit its use. Agar and agarose gels require a simpler — and therefore more reproducible - preparation procedure, making them the better choice for a stable phantom recipe. Variation in the composition of the agar make it a less precise and reliable $R_{2}$ modifier. While agarose is preferable for control of $R_{2}$, agar's reduced price, increased availability, and good long term stability make it a viable alternative. Determining the best gel is a subjective endeavour, and will vary by application. For example, although 


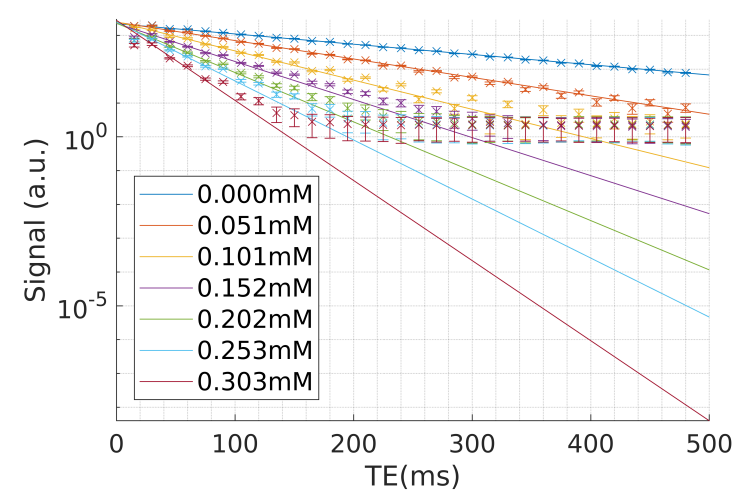

(a) $\mathrm{MnCl}_{2}$ July 16

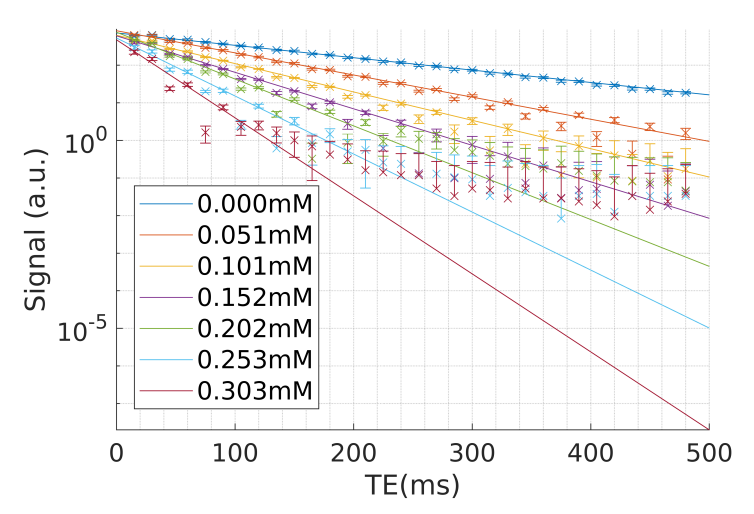

(c) $\mathrm{MnCl}_{2}$ Nov 17

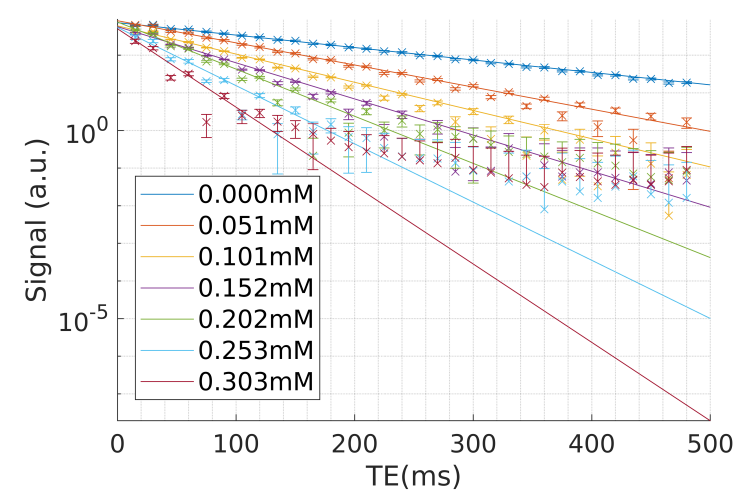

(b) $\mathrm{MnCl}_{2} \mathrm{Nov} 17$

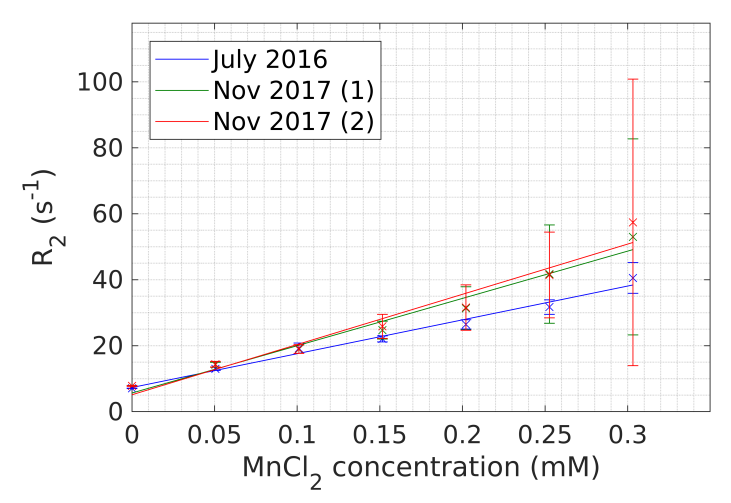

(d) $\mathrm{MnCl}_{2} R_{2}$ relaxivity

Figure 3.11: $T_{E}$ plots and resulting relaxivity estimation for $\mathrm{MnCl}_{2}$. The multiple $T_{E}$ plots relate to multiple acquistion instances.

agarose was able to produce relaxivity fits with high $R^{2}$, it will inevitably vary more with respect to $R_{2}$ due to it's higher $r_{2}$ relaxivity, making it unsuitable for some applications.

Both $\mathrm{MnCl}_{2}$, and $\mathrm{Gd}_{2} \mathrm{O}_{3}$ display consistent $r_{1}$ relativities over extended periods of storage, with $R^{2}$ exceeding 0.94 in all cases. However, $\mathrm{MnCl}_{2} r_{2}$ relaxivity does reveal some temporal instability, with gradient estimations increasing from $102.6 \mathrm{~s}^{-1} \mathrm{mmol}^{-1}$ in July 2016, to around $150 \mathrm{~s}^{-1} \mathrm{mmol}^{-1}$ in November 2017. Degradation of the gels prevents a similar analysis from being performed with the $\mathrm{Gd}_{2} \mathrm{O}_{3}$, so further work is needed to explore this contrast agent.

Further work can be done in this area. The reproducibility of the gel creation process, while apparent in the relaxivity, is not studied in great depth here. To expand on this 
(a) $R_{1}$ relaxivity, $r_{1}$.

\begin{tabular}{|c|c|c|}
\hline Agent & $r_{1} \mathrm{~s}^{-1} \mathrm{mmol}^{-1}$ & $R^{2}$ \\
\hline Agar & $0.0354(0.0166,0.0543)$ & 0.8721 \\
Agarose & $0.0222(0.0159,0.0286)$ & 0.9594 \\
PVA & $0.0380(0.0188,0.0571)$ & 0.9984 \\
$\mathrm{Gd}_{2} \mathrm{O}_{3}$ Sept 16 & $197.1(152.7,241.4)$ & 0.9516 \\
$\mathrm{Gd}_{2} \mathrm{O}_{3}$ Jan 18 & $195.1(149.6,240.7)$ & 0.9483 \\
$\mathrm{Gd}_{2} \mathrm{O}_{3}$ Jan 18 & $177.8(138,217.6)$ & 0.9635 \\
$\mathrm{MnCl}_{2}$ July 16 & $12.48(10.37,14.59)$ & 0.9788 \\
$\mathrm{MnCl}_{2}$ Nov 17 & $11.64(9.059,14.22)$ & 0.9641 \\
$\mathrm{MnCl}_{2}$ Nov 17 & $11.79(9.185,14.39)$ & 0.9644 \\
\hline \multicolumn{2}{|l}{}
\end{tabular}

(b) $R_{2}$ relaxivity, $r_{2}$.

\begin{tabular}{|c|c|c|}
\hline Agent & $r_{2} \mathrm{~s}^{-1} \mathrm{mmol}^{-1}$ & $R^{2}$ \\
\hline Agar & $2.522(-0.0686,5.113)$ & 0.6462 \\
Agarose & $6.583(6.07,7.096)$ & 0.9969 \\
PVA & $0.7276(-1.254,2.71)$ & 0.9561 \\
$\mathrm{Gd}_{2} \mathrm{O}_{3}$ & $252.8(216.2,289.3)$ & 0.9795 \\
$\mathrm{MnCl}_{2}$ July 16 & $102.6(87.23,117.9)$ & 0.9834 \\
$\mathrm{MnCl}_{2}$ Nov 17 & $143.4(116,170.9)$ & 0.9731 \\
$\mathrm{MnCl}_{2}$ Nov 17 & $152.3(114.3,190.3)$ & 0.9551 \\
\hline
\end{tabular}

Table 3.1: $R_{1}$ and $R_{2}$ relaxivities for the pure gels and contrast agent series. relaxivities are reported in $\mathrm{s}^{-1} \mathrm{mmol}^{-1}$, with $95 \%$ confidence intervals, and $R^{2}$ fit errors.

work, multiple independent copies of a particular gel concentrations should be made and characterised. This phantom set could then be used to quantify the temporal stability of the gels, with repeated rescans at regular time intervals. This would also facilitate the determination of effective shelf life of the gel phantoms. There are also other contrast agents that have been used in literature, for example, sodium chloride, and copper sulphate, and carrageen as an alternative gelling agent. 


\section{$4 \quad T_{1}$ mapping: what are we measuring?}

\subsection{Introduction}

Tissue relaxation rates are of fundamental importance in magnetic resonance, as the primary source of image contrast in conventional MRI. In addition to $T_{1}$ and $T_{2}$ weighted imaging [56] $(\mathrm{T} 1 \mathrm{w}, \mathrm{T} 2 \mathrm{w})$, differences in longitudinal $\left(R_{1}=1 / T_{1}\right)$ relaxation rates are also exploited to suppress unwanted signals, e.g. to null the signal from water or fat proton using inversion recovery sequences [57]. Although these techniques use relaxation weighting, optimal choice of sequence parameters inherently requires knowledge of tissue relaxation rates [58]. Absolute quantification of relaxation rates can also be advantageous when compared to relaxation weighted techniques to minimize or eliminate potentially confounding effects of other variables such as variations in proton density or hardware factors such as field inhomogeneity or coil sensitivity $[34,59,60]$.

Relaxation times are also biomarkers in their own right. Quantification of $R_{1}$, which is the main focus of this chapter, has many applications including cardiac $R_{1}$ mapping $[61,62]$ for myocardial pathology [63], liver fibrosis [64] and cirrhosis [65]. Other important applications for $R_{1}$ mapping include dynamic contrast enhanced (DCE) MRI, a technique which aims to model the uptake of an injected contrast agent over time by observing the change in $R_{1}$. The Tofts model [66], used in the prostate [67] for example, requires $R_{1}$ mapping in order to generate uptake information. A related application is the assessment of the safety of Gadolinium-based contrast agents, which has recently been called into question due to evidence of $\mathrm{Gd}$ deposition in tissue such as skin, bone and brain $[68,69]$. Accurate $R_{1}$ mapping could be a useful tool for non-invasively investigating Gd deposition over time, periodically mapping tissue $R_{1}$ values to quantify changes in relaxation rate.

However, a major issue is the large discrepancy in $R_{1}$ values reported in different studies. A recent review by Bojorquez et al. shows that reported $R_{1}$ values for particular tissue types vary greatly. While grey and white matter remain contrasted, the reported $R_{1}$ can still vary by as much as a factor of two [10]. Some differences in measured $R_{1}$ rates may be due to physiological differences in the volunteer populations studied, a problem compounded by the small populations sizes in many studies. Furthermore, while $R_{1}$ rates should in theory 
be intrinsic tissue parameters, there are many potential external factors that can affect the results from hardware considerations such as the field strength of the scanner [70,71], to the choice of mapping sequences and analysis procedures [72]. With regard to the former, multi-site studies by Deoni et al. and Bane et al. found that for a given cohort of volunteers and a fixed acquisition and analysis protocol, the intra-site variation of $R_{1}$ and $R_{2}$ values is on the same order as inter-site variation $[32,73]$.

The focus of this study is to examine the latter source of variation, the effect of the $R_{1}$ mapping protocols. While a wide range of $R_{1}$ mapping protocols have been used in published studies, the majority of them fall in one of three categories, which will be compared in this chapter: inversion recovery (IR), saturation recovery (SR) and fast acquisition variable flip angle methods (VFA). $R_{1}$ quantification using IR mapping is often considered the most accurate method for $R_{1}$ quantification, followed by saturation recovery methods [10]. However, both of these methods require repeated acquisitions while varying $T_{I}$ or $T_{R}$ respectively, rendering them time consuming and impractical for most clinical applications. This has lead to the development of fast acquisition methods based on varying the flip angle. One of the first, the Look-Locker inversion recovery sequence [74], decreases the acquisition time of a standard inversion recovery sequence by applying several low flip angle pulses to enable multiple signal acquisitions within a single repetition time. More recent VFA methods [75, 76] reduce acquisition times even with ultra-fast spoiled gradient echo measurements.

The goal of this study is to compare three major types of $R_{1}$ mapping sequences and assess to which extent variations in the measured $R_{1}$ values can be attributed only to different acquisition protocols. To achieve this, different acquisition protocols are tested for $R_{1}$ contrast phantoms and a small cohort of three volunteers under controlled conditions designed to minimize effect of other factors such as hardware issues or physiological differences.

\subsection{Theory}

Saturation recovery was covered in detail in the previous chapter, so will be omitted from this section. The basis for inversion recovery and variable flip angle methods will be introduced here. The IR pulse sequence (Fig. 4.1) begins with an $180^{\circ}$ inversion pulse, then after time $T_{I}$, a $90^{\circ}$ pulse is applied. Phase and frequency encoding is applied, along with a refocussing 
pulse, with the spin echo formed at time $T_{E}$ after the $90^{\circ}$ pulse. During $T_{I}$, the system will undergo $T_{1}$ relaxation, returning back from inversion to equilibrium. At some point during this process, $M_{z}$ will be approximately zero. If the $90^{\circ}$ pulse is applied at this time, the system will not produce a signal during acquisition. This effectively allows certain tissues to be suppressed based on their $T_{1}$. Inversion recovery is often considered the "gold standard" for $T_{1}$ quantification, superior to saturation recovery, due to its larger dynamic range.

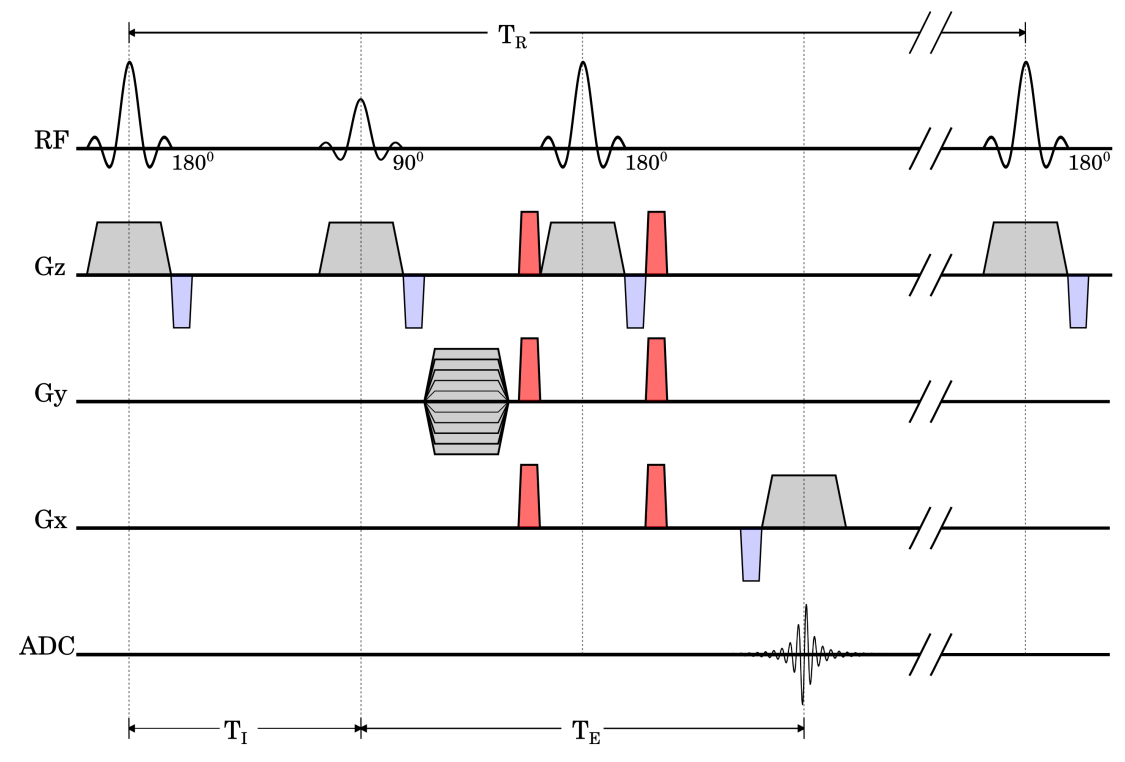

Figure 4.1: Inversion recovery pulse sequence diagram.

$R_{1}$ quantification using IR, again requires repeated acquisition of the sequence, this time with $T_{I}$ varied. Consider a pair of unique pulses with flip angle $\theta_{1}$, and $\theta_{2}$, separated by an inversion time, $T_{I}$. The $z$-magnetisation before $\theta_{2}$ determines the observed signal at readout:

$$
M_{z}\left(\theta_{2}\right)=M_{0} \frac{1-\left(1-\cos \theta_{1}\right) e^{-T_{I} / T_{1}}-\cos \theta_{1} e^{-T_{R} / T_{1}}}{1-\cos \theta_{1} \cos \theta_{2} e^{T_{R} / T_{1}}} .
$$

If $\theta_{1}$ is assumed to be $90^{\circ}$, and $\theta_{2}$ is set to $180^{\circ}$, Eq. (4.1) becomes:

$$
M_{x y}=S_{0}\left(1-r e^{T_{I} / T_{1}}+e^{T_{R} / T_{1}}\right),
$$

where $r$ is some error factor resulting from imperfect refocussing pulses, and $M_{x y}$ is extracted from the dicom signal intensity, $S_{0}, r$, and $T_{1}$ may be determined as fit coefficients. The $e^{T_{R} / T_{1}}$ term is a constant, as $T_{R}$ is fixed. Repeated acquisitions with a variety of $T_{I}$ 's will build the characteristic "bounce curve" of IR relaxometry, shown in Fig. 4.4. 
The VFA methods consist of two or more acquisitions of a spoiled gradient echo sequence (Fig. 2.5), with fixed $T_{R}$ and $T_{E}$. In this analysis method, it is the applied flip angle, $\alpha$, that is varied. For a fixed $T_{R}$ and $T_{E}$ we can write the transverse magnetisation as:

$$
M_{x y}(\alpha)=S_{0} \frac{\sin \alpha}{1-E \cos \alpha},
$$

with $E=\exp \left(-T_{R} / T_{1}\right)$ we can define a system of linear equations in $S_{0}$ and $E$, defining an error vector, e.

$$
\mathbf{e}=\left(\begin{array}{cc}
\sin \alpha_{1} & M_{x y}\left(\alpha_{1}\right) \cos \alpha_{1} \\
\vdots & \vdots \\
\sin \alpha_{n} & M_{x y}\left(\alpha_{n}\right) \cos \alpha_{n}
\end{array}\right)\left(\begin{array}{c}
S_{0} \\
E
\end{array}\right)-\left(\begin{array}{c}
M_{x y}\left(\alpha_{1}\right) \\
\vdots \\
M_{x y}\left(\alpha_{n}\right)
\end{array}\right)
$$

If there are exactly $m=2$ different flip angles then there is generally a unique solution with $\mathbf{e}=\mathbf{0}$. For $m>2$ the system is overdetermined and we minimize the least-squares error or $L_{2}$ norm of the error vector e to determine $S_{0}$ and $E$ from which we can infer $R_{1}=-\ln (E) / T_{R}$. VFA methods are much faster than SR and IR, requiring as few as two acquisitions. However, the $T_{1}$ estimation requires a uniform flip angle across the entire region of interest, so as to attribute any signal variation solely to $T_{1}$. This is difficult to achieve, as $B_{1}$ inhomogeneity will always be present to some degree. For this study, all VFA scans were conducted on uniform agar phantoms to reduce the effect of $B_{1}$ inhomogeneity

\subsection{Materials and Methods}

\subsubsection{Test objects and subjects}

A concentrated solution of manganese chloride was made by dissolving $0.1 \mathrm{~g}$ manganese chloride tetrahydrate $(98 \%)$ in 11 of deionized water. The concentrate was then further diluted, to varying degrees, to create a series of seven $100 \mathrm{ml}$ solutions with manganese chloride concentrations of $0,10,20,30,40,50$, and $60 \mathrm{mgl}^{-1}$, where $10 \mathrm{mg} \mathrm{l}^{-1}$ corresponds to a $0.0505 \mathrm{mmol}$ concentration of $\mathrm{MnCl}_{2}$. This range was selected - following a preliminary investigation - to maximise the range of concentrations, while maintaining acceptable signal.

After scanning the solutions using the protocols described in Sec. 4.3.2, $1 \mathrm{~g}$ of agar was added to each solution, to create $1 \%$ agar gels. $0.1 \mathrm{~g}$ of diazolidinyl urea was also added as 
a preservative at this stage. The solutions were heated to approximately $90^{\circ} \mathrm{C}$ and stirred until the agar was fully dissolved and then allowed to cool and set overnight before being rescanned using identical protocols.

To obtain in vivo data, three healthy volunteers, two males and one female aged 24-32, were recruited to undergo brain scans with different $R_{1}$ mapping protocols. One volunteer returned for a second session the following day, where the protocol was repeated. This provided an opportunity to compare intra, and inter-participant variation.

\subsubsection{MR Protocols}

All scans were conducted on a Siemens 3T magnetom Skyra scanner at Swansea university. The scanner is situated in an air-conditioned environment kept at a temperature of $20^{\circ} \mathrm{C}$. Manual shimming and transmitter calibration were performed prior to all data acquisition scans to minimize effects of $B_{0}$ and $B_{1}$ inhomogeneity.

Phantom scans The seven phantoms were placed in a hexagonal arrangement around the isocentre of the magnet above the four channel spine coil element SP2. Again, SP2 was selected to maximise the reproducibility of the phantom setup. For the IR and SR protocols, a single $2 \mathrm{~mm}$ thick coronal slice through the centre of the phantoms was selected and all images were acquired with a $256 \times 256 \mathrm{~mm}$ field of view (FOV), matrix size $128 \times 128$, pixel bandwidth $130 \mathrm{~Hz} / \mathrm{px}, 100 \%$ phase resolution, and one average. The SR protocol consisted of acquiring a series of images with a vendor-supplied 2D spin echo (SE) sequence, comprised of a $90^{\circ}$ excitation pulse followed by a $180^{\circ}$ refocusing pulse with fixed $T_{E}$ of $12 \mathrm{~ms}$, and $T_{R}$ $=75 \mathrm{~ms}, 125 \mathrm{~ms}, 250 \mathrm{~ms}, 500 \mathrm{~ms}, 750 \mathrm{~ms}, 1000 \mathrm{~ms}, 1500 \mathrm{~ms}, 2000 \mathrm{~ms}, 3000 \mathrm{~ms}, 4000 \mathrm{~ms}$ and $5000 \mathrm{~ms}$. For the IR protocol a vendor-supplied 2D SE sequence with an additional inversion pulse prior to the excitation pulse was used, with a fixed $T_{R}$ of $2500 \mathrm{~ms}, T_{E}$ of $15 \mathrm{~ms}$, and $T_{I}$ values of $30 \mathrm{~ms}, 70 \mathrm{~ms}, 100 \mathrm{~ms}, 150 \mathrm{~ms}, 300 \mathrm{~ms}, 700 \mathrm{~ms}, 1000 \mathrm{~ms}, 1500 \mathrm{~ms}$ and $2000 \mathrm{~ms}$. The VFA protocol consisted of applying a vendor-supplied 3D spoiled gradient echo (GE) sequence with $T_{R}=4.09 \mathrm{~ms}$ and $T_{E}=1.39 \mathrm{~ms}$ for a range of flip angles between $2^{\circ}$ and $90^{\circ}$. Twenty $2 \mathrm{~mm}$ thick coronal slices were acquired and a slice through the centre, matching the slice chosen for the SR/IR protocols, was selected for the analysis. The FOV was $256 \times$ 
$256 \mathrm{~mm}$, matching the IR/SR protocol, while for the matrix size and readout bandwidth, the default values from a clinical protocol of $192 \times 154$ and $390 \mathrm{~Hz} / \mathrm{px}$, respectively, were used. One series was also acquired with a longer $T_{R}$ of $14 \mathrm{~ms}$ for comparison. For each flip angle 8 averages were acquired.

In vivo scans All volunteers were scanned using identical protocols in a 32 channel phasedarray head coil, this choice provided the best SNR for whole-brain imaging. To ensure coverage of the entire head, 21 slices with of thickness and spacing of $5 \mathrm{~mm}$ were acquired. The imaging plane was offset from transverse to coronal by $25^{\circ}$. The FOV was $192 \times 256$ and the matrix size $128 \times 96$. 96 phase encoding steps and a per pixel bandwidth of $130 \mathrm{~Hz} / \mathrm{px}$. The SR protocol used a vendor-supplied SE sequence with fixed $T_{E}$ of $15 \mathrm{~ms}$ and $T_{R}$ of 286 , 350, 550, 885, 1000, 2000, $4000 \mathrm{~ms}$. The IR protocol used a vendor-supplied SE sequence with an additional inversion pulse with $T_{R}=2500 \mathrm{~ms}, T_{E}=15 \mathrm{~ms}$ and inversion times of 100, 400, 700, 1000, 1300, and $1600 \mathrm{~ms}$.

\subsection{3 $\quad R_{1}$ estimation}

In-house developed Matlab software was used to analyze the scan data. For the phantom data, delineation of the regions of interest (ROIs) was automated by thresholding the signal from one reference image, identifying the connected components, and fitting circular ROIs for each component. Approximately $10 \%$ of the phantom's extent was excluded so as to limit signal variation at the interface with the phantom's container, and both single voxel and mean-signal-over-ROI fits were performed. For the brains, square ROIs corresponding to the cerebellum and thalamus were selected manually, as illustrated in Fig. 4.2. Grey and white matter regions were identified for a representative slice in the centre of each volunteer's brain. For this slice, the thalamus was manually identified and removed from analysis, along with boundary regions with the skull. The remaining voxels were assumed to be some distribution of grey and white matter. A single voxel analysis was performed on each phantom, ROI, and representative slice, resulting in an $R_{1}$ value for each voxel in the region. Fig. 4.3 shows the $R_{1}$ distributions for an image, IR $R_{1}$ map, and an SR $R_{1}$ map for one of the representative slices. The IR maps exhibit a bi-modal distribution in $R_{1}$, with the modes corresponding 
to the $R_{1}$ distributions of grey and white matter respectively. Image processing - using a combination of thresholding and connected component analysis - was applied to the IR maps in order to separate grey and white matter regions for independent analysis.
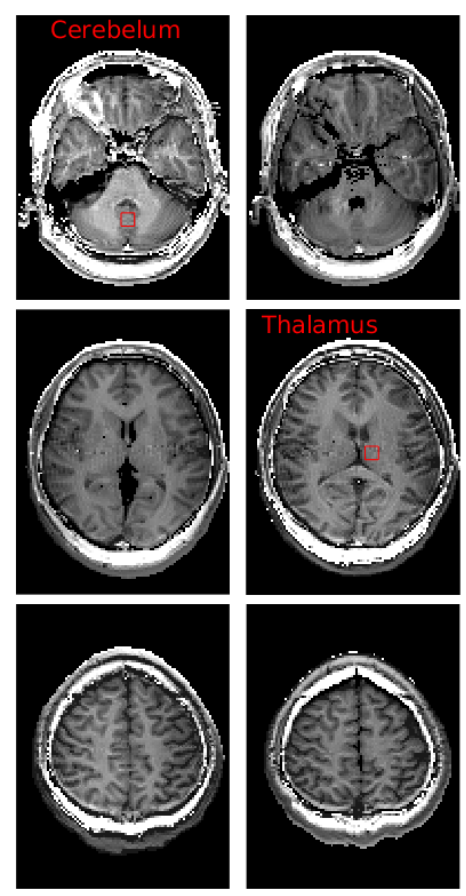
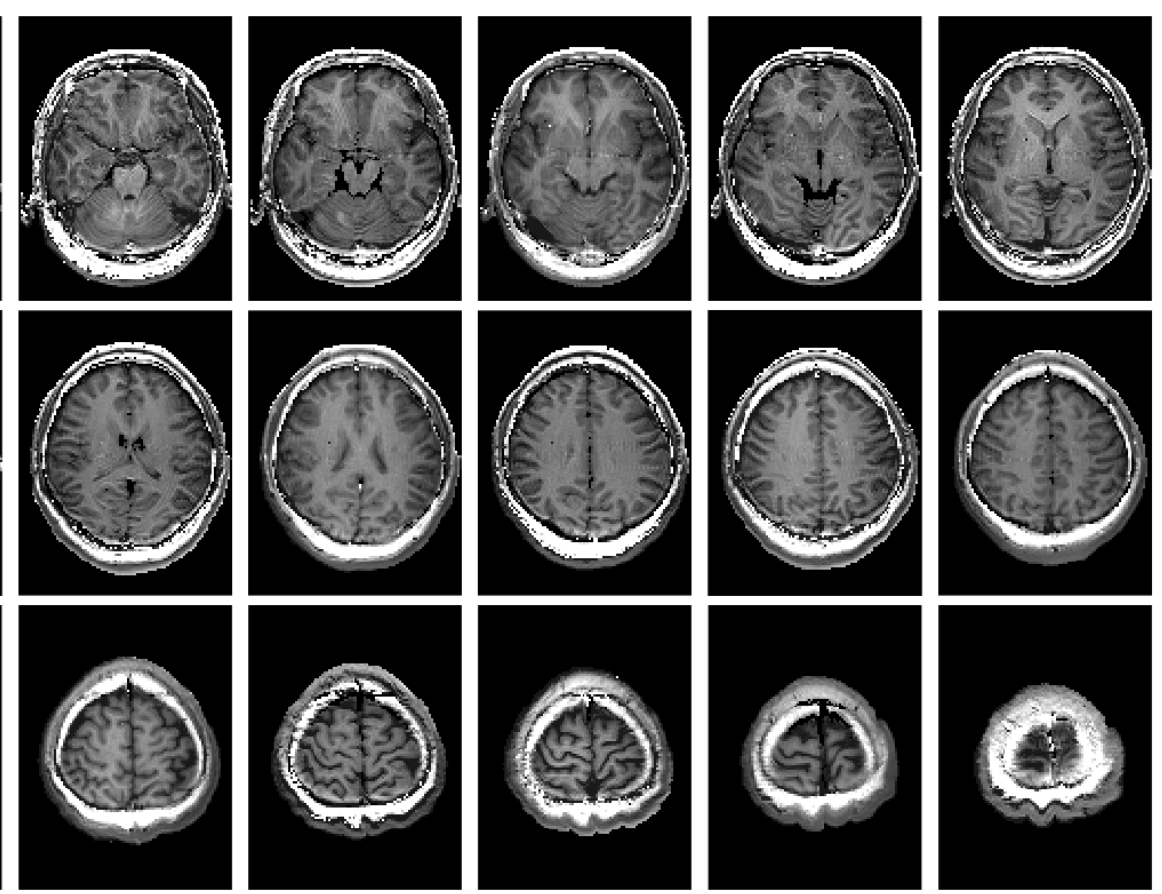
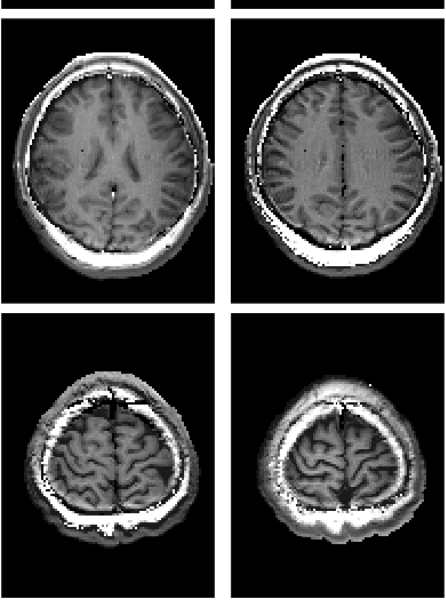
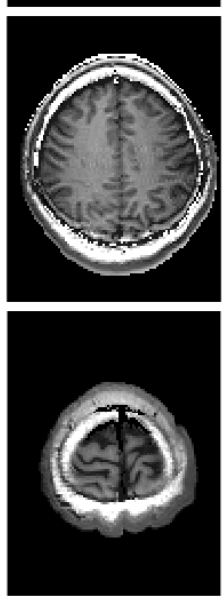
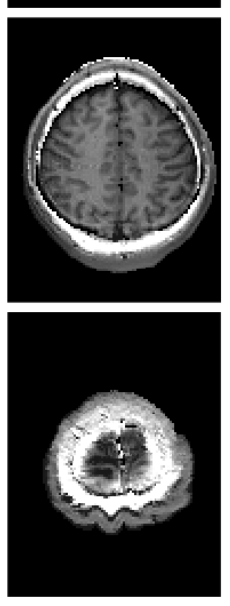

Figure 4.2: Delineated regions of interest for one of the patient cohort. One slice is selected to delineate the cerebellum, and a second for the thalamus.

SR and IR curve fitting was carried out with a trust-region-reflective least-squares minimisation algorithm, using the Matlab curve fitting toolbox with custom fit functions defined according to Eq. (3.1) and Eq. (4.2) respectively. The quality of each fit is assessed using the $R^{2}$ value. The VFA fit quality was assessed by determining the $L_{2}$ norm of $\mathbf{e}$ in Eq. (4.4).

\subsubsection{Statistical Analysis}

For statistical purposes, single voxel fits were performed for each voxel inside the selected ROIs for both phantom and brains. This results in a distribution of $R_{1}$ values for each ROI. Assuming the values are normally distributed, we can calculate a mean and standard deviation of $R_{1}$ for each ROI. We test the distribution for normality by calculating the Kolmogorov-Smirnov [77] score. The overlap between the histograms for different ROIs yields 


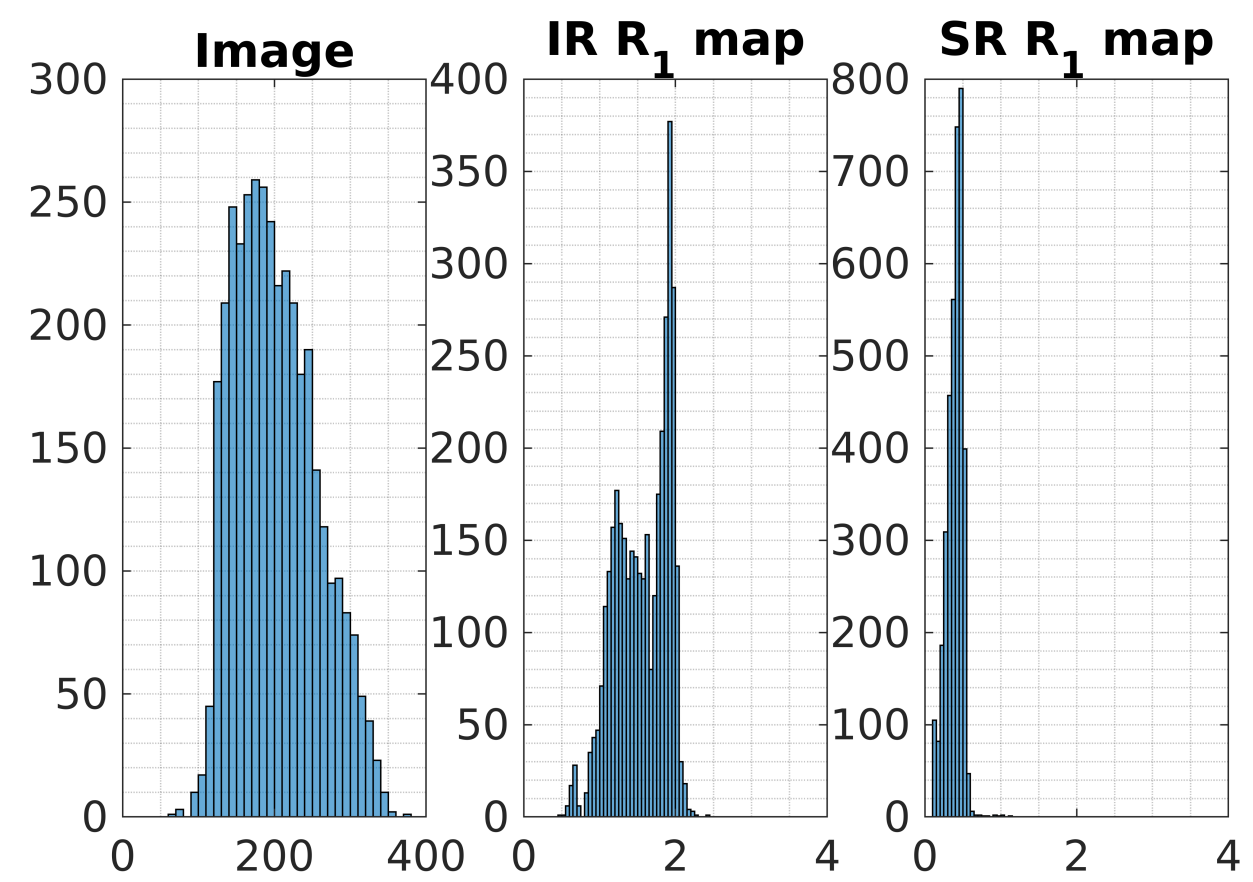

(a) Histogram of contrast distributions for image and $R_{1}$ maps for a single volunteer and slice.

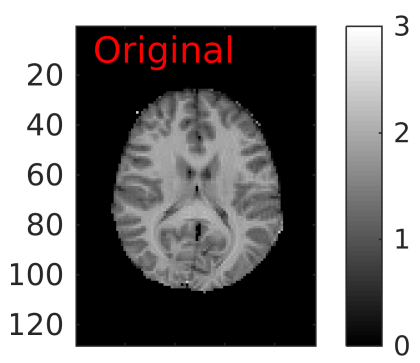

20406080

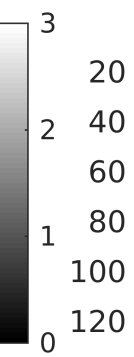

120

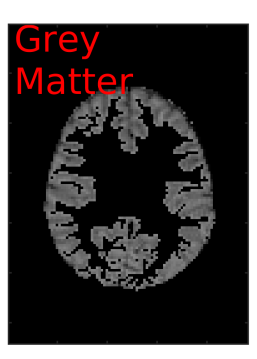

20406080

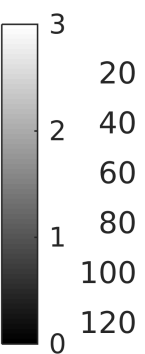

120

20406080

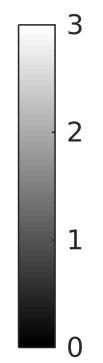
1

(b) Example of grey and white matter delineation for one volunteer.

Figure 4.3: The only histogram that displays a bi-modal distribution of $R_{1}$ values is IR.

Hence this was used for delineation. The thalamus region was manually excluded, and the grey and white matter separated using image processing.

information about how distinguishable the $R_{1}$ values for different ROIs are. To establish whether there is a statistically significant difference between the means in the $R_{1}$ distributions obtained for the same phantom or brain ROI with different $R_{1}$ mapping methods, a twosample t-test [78] is performed and $p$-value and confidence interval for the estimated difference 
in the means for the $R_{1}$ distributions is determined.

For the $R_{1}$ contrast phantoms, both liquids and gels, $R_{1}$ should increase linearly with the concentration of the contrast agent. Therefore, we also plot the mean and standard deviation of the $R_{1}$ values as a function of the concentration of manganese chloride and perform a linear fit to determine the slope of the line, which corresponds to the relaxivity of the contrast agent, enabling us to compare not only the $R_{1}$ value, but also the resulting relaxivity of contrast agent obtained for different $R_{1}$ mapping methods.

\subsection{Results}

The $R_{1}$ values for the liquid and gel phantoms obtained with different methods are tabulated in Tables 4.1 and 4.2. The mean and standard deviation were calculated from the distributions of the single voxel fits for each phantom. The tables clearly show significant variation in the $R_{1}$ values obtained for the same phantom using different methods. To further elucidate the magnitude and significance of the differences in the $R_{1}$ means, we performed pairwise t-tests on the $R_{1}$ distributions obtained with different methods for the same phantom. The results tabulated in Table 4.3 .

Comparing the SR and IR protocols, the differences in the means of the distributions were larger for phantoms with lower concentrations of $\mathrm{MnCl}_{2}$. For the VFA method, the differences in the $R_{1}$ means for different VFA protocols were smaller, but they differed significantly from the estimates obtained with the SR and IR protocol. For phantoms with low concentrations of $\mathrm{MnCl}_{2}$ the $R_{1}$ values obtained for the VFA methods, especially the common two-point method with flip angles $2^{\circ}$ and $15^{\circ}$, were much closer to SR values than IR values, while the situation was reversed for higher concentrations of the contrast agent.

Non-negligible differences in the $R_{1}$ values obtained for the same phantom are also evident in the relaxivity plots in Figure 4.5, which show that the $R_{1}$ values obtained for the IR protocol are consistently higher than the corresponding values obtained with the SR protocol, although the differences decrease slightly with increasing contrast agent concentration. This is the case for both the liquid and gel phantoms although the differences for the gel phantom are somewhat smaller. Similarly, the $R_{1}$ values for the two-point VFA method start close to the values for the $\mathrm{SR}$ protocol for low concentrations of $\mathrm{MnCl}_{2}$ but for high concentrations 


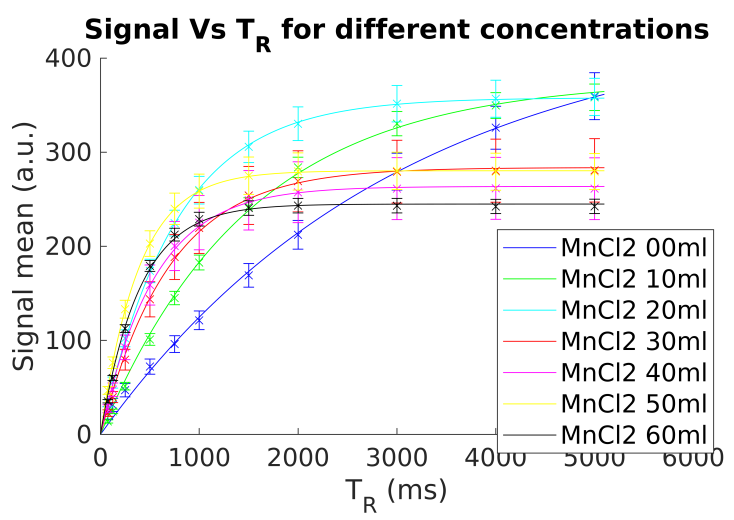

(a) SR - solution

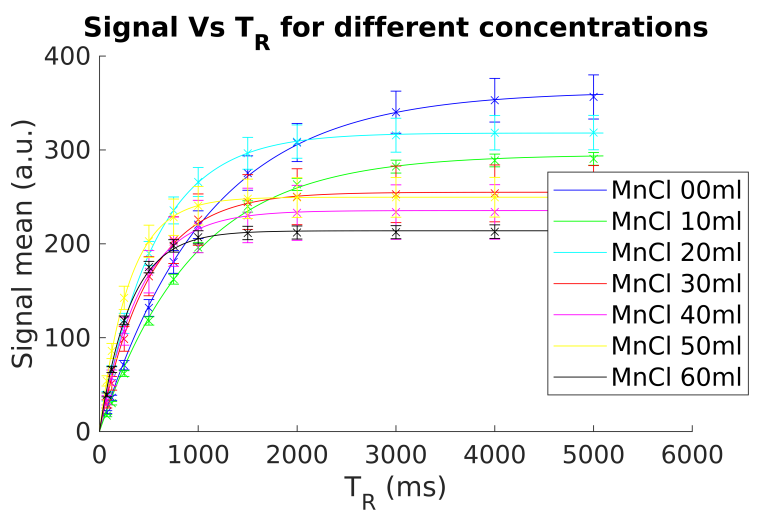

(c) SR - gel

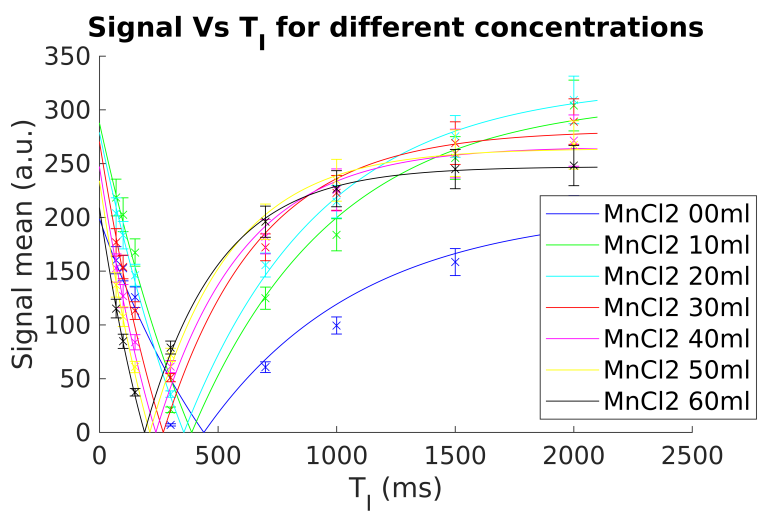

(b) IR - solution

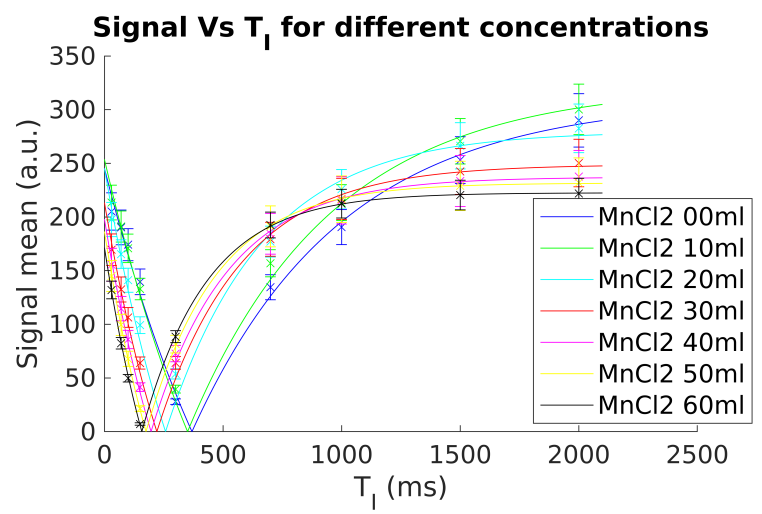

(d) IR - gel

Figure 4.4: Relaxivity curves for $\mathrm{MnCl}_{2}$ solutions and gels

exceed the $R_{1}$ values obtained for the IR method. These differences affect the slope of the linear regression line, which corresponds to the relaxivity of the contrast agent, resulting in different estimates for the relaxivity (see Table 4.4) although the overlap of the 95\% confidence intervals of the estimates suggests that data for more phantoms with possibly a wider range of concentrations would be required ascertain if the differences in relaxivity estimates are significant at the $95 \%$ confidence level.

Further details can be found in Appendix B. Figures B.2 and B.4 show the IR and SR image series with ROIs selected and the $R_{1}$ map obtained for the liquid and gel phantoms, respectively. Fig. B.1 shows the images for an ultrafast flip angle series with $T_{R}=4.09 \mathrm{~ms}$ and the corresponding $R_{1}$ maps obtained using two and nine flip angles, respectively. For the IR and SR protocols, the corresponding transverse magnetization signal (mean and 


\begin{tabular}{|c|c|c|c|c|c|c|c|c|}
\hline & \multicolumn{2}{|c|}{ IR - solution } & \multicolumn{2}{|c|}{ SR - solution } & \multicolumn{2}{|c|}{ IR - gel } & \multicolumn{2}{|c|}{ SR - gel } \\
\hline $\begin{array}{l}\text { Conc } \\
(\mathrm{mmol})\end{array}$ & $\begin{array}{c}\overline{R_{1}} \\
(\mathrm{~Hz})\end{array}$ & $\begin{array}{c}\sigma \\
(\mathrm{Hz})\end{array}$ & $\begin{array}{c}\overline{R_{1}} \\
(\mathrm{~Hz})\end{array}$ & $\begin{array}{c}\sigma \\
(\mathrm{Hz})\end{array}$ & $\begin{array}{c}\overline{R_{1}} \\
(\mathrm{~Hz})\end{array}$ & $\begin{array}{c}\sigma \\
(\mathrm{Hz})\end{array}$ & $\begin{array}{c}\overline{R_{1}} \\
(\mathrm{~Hz})\end{array}$ & $\begin{array}{c}\sigma \\
(\mathrm{Hz})\end{array}$ \\
\hline 0.0000 & 1.5332 & 0.033396 & 0.27012 & 0.017031 & 1.5798 & 0.019794 & 0.95521 & 0.027487 \\
\hline 0.0505 & 1.6774 & 0.027502 & 0.70731 & 0.029947 & 1.683 & 0.11854 & 1.1378 & 0.039074 \\
\hline 0.1011 & 1.7417 & 0.018924 & 1.2977 & 0.055165 & 2.4426 & 0.029179 & 1.8228 & 0.070709 \\
\hline 0.1516 & 2.5027 & 0.029774 & 1.5187 & 0.036961 & 2.8013 & 0.039291 & 2.2092 & 0.078524 \\
\hline 0.2021 & 2.7653 & 0.035631 & 1.9646 & 0.048536 & 3.0858 & 0.0418 & 2.6745 & 0.10494 \\
\hline 0.2526 & 2.9866 & 0.04034 & 2.6034 & 0.096198 & 3.3651 & 0.045296 & 3.3335 & 0.13628 \\
\hline 0.3032 & 3.219 & 0.045863 & 2.7624 & 0.07993 & 3.6218 & 0.066539 & 3.5571 & 0.43442 \\
\hline
\end{tabular}

Table 4.1: Mean values of single voxel $R_{1}$ fits, $\overline{R_{1}}$, and standard deviation, $\sigma$ obtained via Inversion recovery (IR) and saturation recovery (SR) for solution and gel $\mathrm{MnCl}_{2}$ phantoms.

\begin{tabular}{|c|c|c|c|c|c|c|c|c|}
\hline \multirow[b]{2}{*}{$\begin{array}{c}\text { Conc } \\
(\mathrm{mmol})\end{array}$} & \multicolumn{2}{|c|}{ 9-Point $T_{R}=4090$} & \multicolumn{2}{|c|}{ 12-Point $T_{R}=14000$} & \multicolumn{2}{|c|}{ 2-Point $T_{R}=4090$} & \multicolumn{2}{|c|}{ 2-Point $T_{R}=14000$} \\
\hline & $\begin{array}{l}\overline{R_{1}} \\
(\mathrm{~Hz})\end{array}$ & $\begin{array}{c}\sigma \\
(\mathrm{Hz})\end{array}$ & $\begin{array}{c}\overline{R_{1}} \\
(\mathrm{~Hz})\end{array}$ & $\begin{array}{c}\sigma \\
(\mathrm{Hz})\end{array}$ & $\begin{array}{c}\overline{R_{1}} \\
(\mathrm{~Hz})\end{array}$ & $\begin{array}{c}\sigma \\
(\mathrm{Hz})\end{array}$ & $\begin{array}{c}\overline{R_{1}} \\
(\mathrm{~Hz})\end{array}$ & $\begin{array}{c}\sigma \\
(\mathrm{Hz})\end{array}$ \\
\hline 0.0000 & 1.0041 & 0.043723 & 0.78626 & 0.097955 & 1.0269 & 0.044152 & 1.0784 & 0.044892 \\
\hline 0.0505 & 1.459 & 0.12399 & 1.2705 & 0.15092 & 1.4792 & 0.12086 & 1.5916 & 0.16318 \\
\hline 0.1011 & 1.6947 & 0.11322 & 1.5305 & 0.14603 & 1.7282 & 0.11675 & 1.816 & 0.11493 \\
\hline 0.1516 & 2.6534 & 0.19661 & 2.5533 & & 2.6694 & 0.2025 & 2.7525 & 0.21346 \\
\hline 0.2021 & 3.0815 & 0.18991 & 3.2119 & 0.22549 & 3.0869 & 0.19886 & 3.2377 & 0.26769 \\
\hline 0.2526 & 3.2353 & 0.17847 & 3.2976 & 0.18677 & 3.2784 & 0.19627 & 3.3791 & 0.22033 \\
\hline 0.3032 & 4.3828 & 0.36785 & 4.4967 & 0.47059 & 4.4158 & 0.3849 & 4.5358 & 0.41844 \\
\hline
\end{tabular}

Table 4.2: Mean values of single voxel $R_{1}$ fits, $\overline{R_{1}}$, and standard deviation, $\sigma$ obtained for VFA methods. For the two-point method flip angles of $2^{\circ}$ and $15^{\circ}$ were used. For the 9point method with $T_{R}=4.09 \mathrm{~ms}$ the flip angles were $2,3,4,6,8,10,12,13$ and 15 degrees. For the 12-point method with $T_{R}=14 \mathrm{~ms}$ the flip angles were 2, 5, 10, 15, 20, 30, 50, 60, 70, 80 and 90 degrees.

standard deviation) as a function of $T_{R}$ and $T_{I}$, respectively, with the best-fit curves, is shown in Figures B.3 and B.5 for different concentrations of the contrast agent, for both liquid and gel contrast phantoms. Although only the mean signal fits are shown, they are representative of the single voxel fits. The quality of the non-linear fits of the saturation and 


\begin{tabular}{|l||c|c|c|c|}
\hline $\mathrm{MnCl}_{2}$ & CI IR-SR Sol & CI IR-SR Gel & CI IR-VFA2 Gel & CI SR-VFA2 Gel \\
\hline $0.0000 \mathrm{mmol}$ & $(0.6215,0.6277)$ & $(1.2596,1.2665)$ & $(0.5568,0.5627)$ & $(-0.0683,-0.0615)$ \\
$0.0505 \mathrm{mmol}$ & $(0.5337,0.5567)$ & $(0.9664,0.9739)$ & $(0.2042,0.2300)$ & $(-0.3358,-0.3204)$ \\
$0.1011 \mathrm{mmol}$ & $(0.6127,0.6269)$ & $(0.4387,0.4494)$ & $(0.7515,0.7626)$ & $(0.1291,0.1454)$ \\
$0.1516 \mathrm{mmol}$ & $(0.5840,0.6002)$ & $(0.9797,0.9884)$ & $(0.1495,0.1735)$ & $(-0.4442,-0.4171)$ \\
$0.2021 \mathrm{mmol}$ & $(0.4008,0.4217)$ & $(0.7952,0.8063)$ & $(0.0101,0.0349)$ & $(-0.4040,-0.3735)$ \\
$0.2526 \mathrm{mmol}$ & $(0.0184,0.0449)$ & $(0.3736,0.3928)$ & $(0.1588,0.1781)$ & $(0.1215,0.1521)$ \\
$0.3032 \mathrm{mmol}$ & $(0.0242,0.1052)$ & $(0.4480,0.4650)$ & $(-0.7717,-0.7251)$ & $(-0.8590,-0.7672)$ \\
\hline
\end{tabular}

Table 4.3: Pairwise t-test results (allowing for differences in variance) for $R_{1}$ distributions obtained with IR, SR and VFA 2-15 protocols for different phantoms show significant difference. CI denotes the confidence intervals for the estimated difference in the means of the two $R_{1}$ distributions. The $p$-values for all tests were $<0.001$, strongly rejecting the null hypothesis that the $R_{1}$ values come from the same distribution.

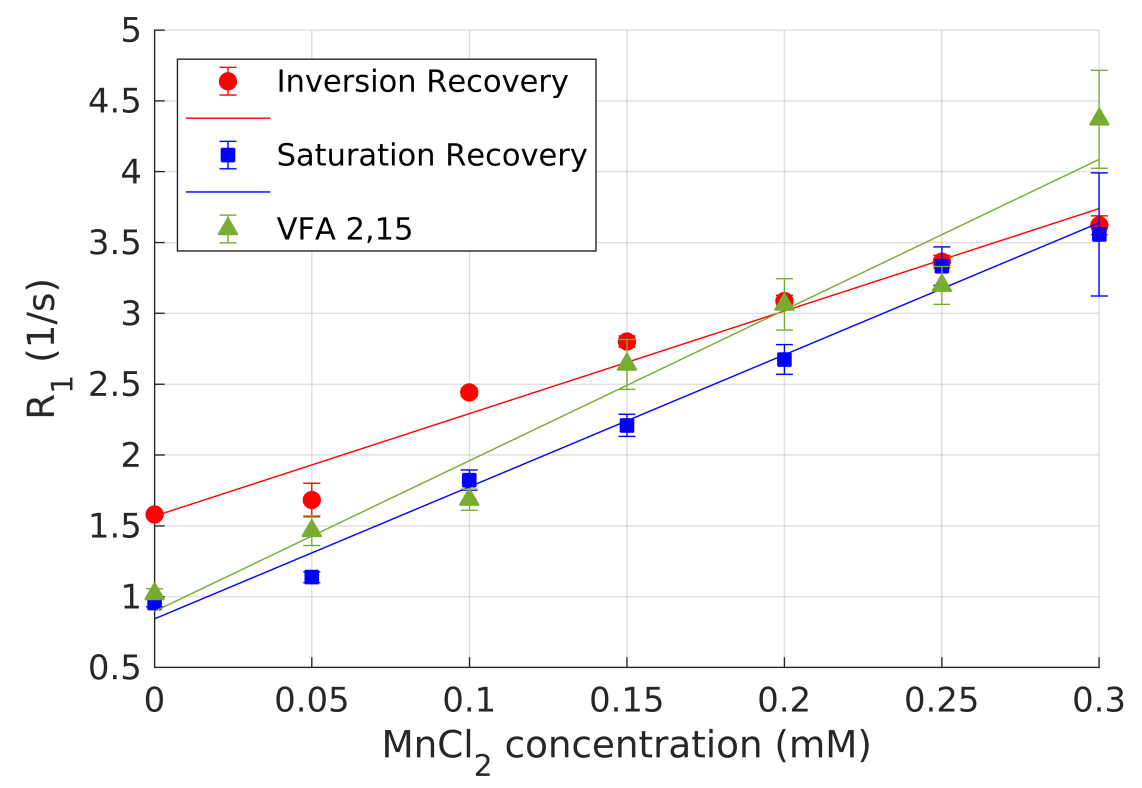

Figure 4.5: Relaxivity plot of $\mathrm{MnCl}_{2}$ gel phantoms comparing IR, SR and 2-15 VFA protocols.

inversion recovery curves obtained was high: the majority of the single voxel fits have $R^{2}$ values $\geq 0.99$, and the percentages of single voxel fits with $R^{2}<0.95$ are low at $1.2 \%, 2.5 \%$, $15.1 \%$ 0.02\% for IR gel fits, SR gel fits, IR solution fits and SR solution fits, respectively.

Although differences in signal intensity are visible in the images, it is difficult to reliably 


\begin{tabular}{|c|c|c|c|}
\hline Protocol & $\begin{array}{c}\text { relaxivity }(95 \% \mathrm{CI}) \\
\mathrm{Hz} \mathrm{mmol}^{-1}\end{array}$ & $\begin{array}{c}y \text {-intercept }(95 \% \mathrm{CI}) \\
\mathrm{Hz}\end{array}$ & $R^{2}$ of fit \\
\hline Solution-IR & $6.155001(4.532139,7.777863)$ & $1.4145(1.1184,1.7106)$ & 0.9498 \\
Solution-SR & $8.430966(7.342461,9.539262)$ & $0.3103(0.1098,0.5108)$ & 0.98729 \\
Gel-IR & $7.164342(5.640435,8.688249)$ & $1.5685(1.2920,1.8449)$ & 0.96715 \\
Gel-SR & $9.222606(8.015355,10.429857)$ & $0.8434(0.6236,1.0631)$ & 0.98722 \\
9-Point & $10.647558(8.292429,13.002687)$ & $0.8863(0.4570,1.3156)$ & 0.96432 \\
12-Point & $11.914182(9.400725,14.447430)$ & $0.6424(0.1837,1.1010)$ & 0.96736 \\
2-Point, $T_{R}=4090$ & $10.687140(8.411175,12.982896)$ & $0.9060(0.4891,1.3229)$ & 0.96649 \\
2-Point, $T_{R}=14000$ & $10.865259(8.549712,13.180806)$ & $0.9806(0.5588,1.4024)$ & 0.96677 \\
\hline
\end{tabular}

Table 4.4: Relaxivity fit coefficients for each phantom scan including $95 \%$ confidence intervals, and $R^{2}$ fit error

distinguish all contrast phantoms based on a single image. The $R_{1}$ maps differentiate the contrast phantoms much better. For all methods tested, all phantoms are mutually distinguishable in that the pairwise t-test for the $R_{1}$ distributions for any two phantoms rejects the null hypothesis that they come from the same distribution at the $p=0.01$ level even when allowing for unequal variances of the distributions.

Analysis of the distribution of $R_{1}$ values for different phantoms and methods in Fig. 4.6, however, shows that there are variations in the shape of the distributions for individual phantoms and the degree of overlap for different phantoms. Considering the overlap between the distributions, the SR protocol appears slightly worse in discriminating the phantoms with the highest concentration of $\mathrm{MnCl}_{2}$ while our IR protocol appears slightly worse for the lower contrast phantoms. Comparing the distributions for the IR and two-point VFA method also shows that the distributions for the latter are much broader with greater overlap of the distributions for different phantoms, which is also reflected in the larger standard deviation of the $R_{1}$ values obtained with VFA methods (see Tables 4.1, 4.2).

Table 4.5 shows a summary of the $R_{1}$ mapping results for different brain regions and four in vivo data sets, corresponding to three different volunteers with volunteer 2 being scanned twice on two consecutive days. IR sequences consistently report a higher $R_{1}$ (lower $T_{1}$ ), irrespective of region or volunteer. Table 4.5 also shows the t-test p-values, a measure 


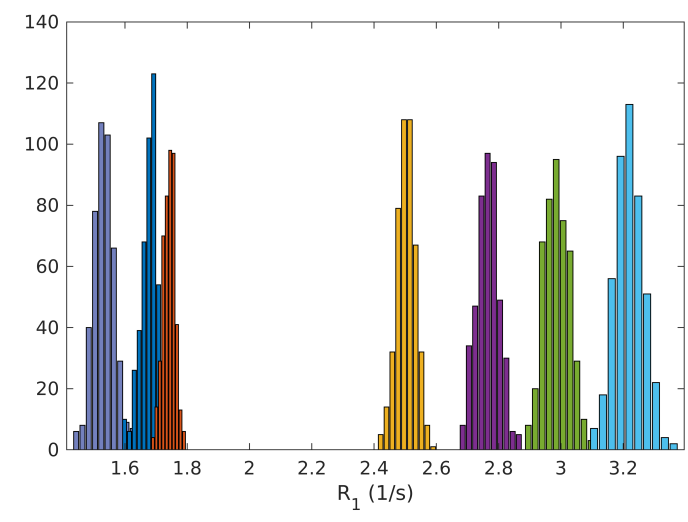

(a) IR Series ( $\mathrm{MnCl}_{2}$ Solutions)

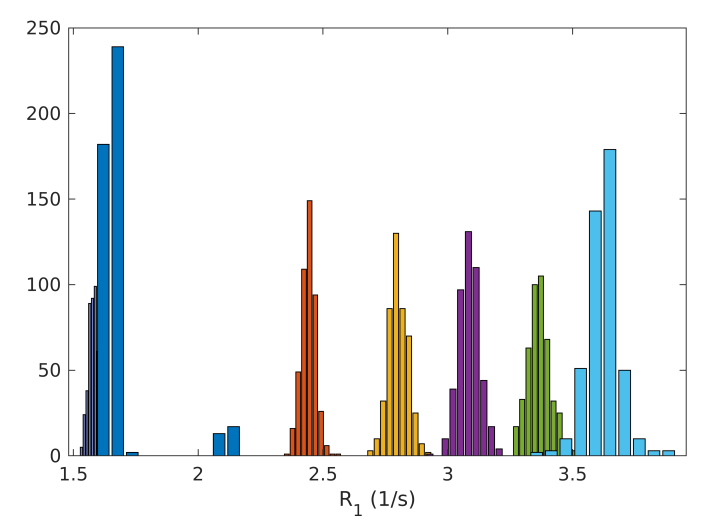

(c) IR Series $\left(\mathrm{MnCl}_{2}\right.$ Gels $)$

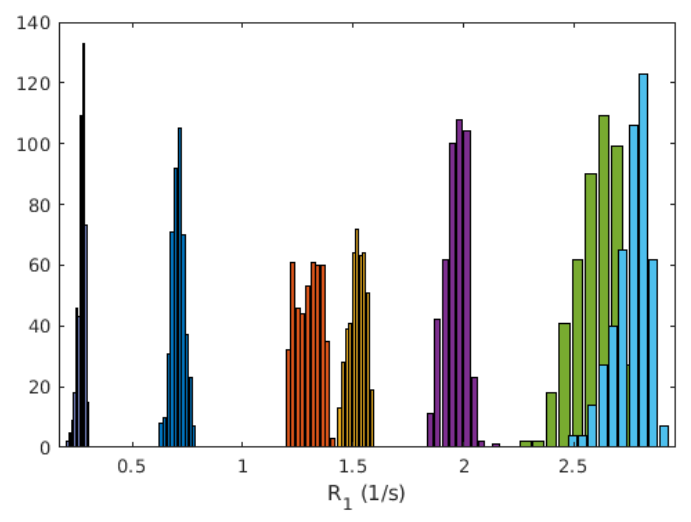

(b) SR Series $\left(\mathrm{MnCl}_{2}\right.$ Solutions)

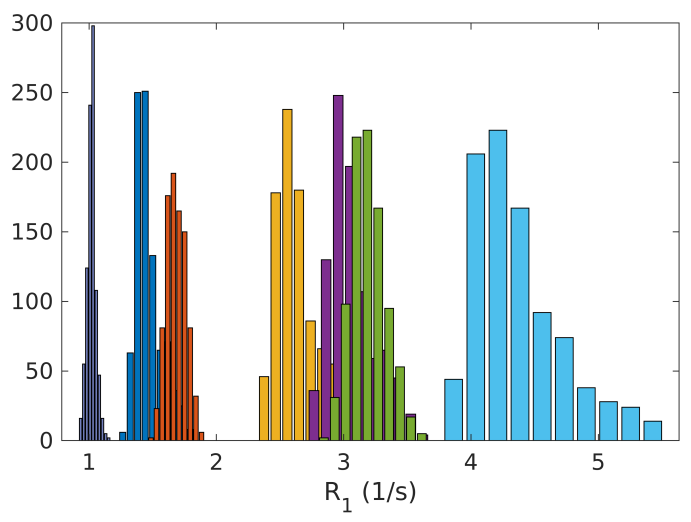

(d) VFA Series $\left(\mathrm{MnCl}_{2}\right.$ Gels)

Figure 4.6: Histogram of $R_{1}$ values for obtained for $\mathrm{MnCl}_{2}$ phantoms with various methods. Each phantom is plotted separately; $0.0000 \mathrm{mmol}$ (light-purple), $0.0505 \mathrm{mmol}$ (dark-blue), $0.1011 \mathrm{mmol}$ (orange), $0.1516 \mathrm{mmol}$ (yellow), $0.2021 \mathrm{mmol}$ (dark-purple), $0.2526 \mathrm{mmol}$ (green), $0.3032 \mathrm{mmol}$ (light-blue).

of the probability of the observed separation being a result of a single distribution. All reported p-values for the in-vivo SR and IR comparisons fell below $4.977 \times 10^{-10}$, indicating a strong separation of $R_{1}$ distributions. IR scans test-retest stability, with $\overline{R_{1}}$ for the repeat patient reproduced to at least one decimal place in all cases. However SR protocols do not exhibit the same stability, with the inter-participant variation on the same order as the intraparticipant variation. Contrast is maintained in both sequences, but absolute quantification of $R_{1}$ appears more stable for the so called "gold standard" IR sequence. 


\begin{tabular}{|c|cc|cc|cc|}
\hline & \multicolumn{2}{|l|}{ Saturation recovery } & \multicolumn{2}{|c|}{ Inversion recovery } & & \\
\hline \multirow{3}{*}{ ROI } & $\overline{R_{1}}$ & $\sigma$ & $\overline{R_{1}}$ & $\sigma$ & t-test p-value & t-test CI \\
\hline \multirow{5}{*}{ Cerebellum } & 0.54049 & 0.05005 & 0.65455 & 0.056634 & $2.3887 \mathrm{e}-13$ & $(0.088926,0.139186)$ \\
& 0.55055 & 0.068363 & 0.7487 & 0.078851 & $1.7832 \mathrm{e}-17$ & $(0.163442,0.232847)$ \\
& 0.57851 & 0.067102 & 0.72479 & 0.062519 & $4.114 \mathrm{e}-14$ & $(0.115542,0.177005)$ \\
& 0.53432 & 0.089759 & 0.68661 & 0.089246 & $4.977 \mathrm{e}-10$ & $(0.110214,0.194364)$ \\
\hline \multirow{3}{*}{ Thalamus } & 0.45608 & 0.061248 & 0.78954 & 0.10613 & $9.7379 \mathrm{e}-22$ & $(0.291310,0.375618)$ \\
& 0.58107 & 0.034651 & 0.91667 & 0.091879 & $2.1845 \mathrm{e}-24$ & $(0.302634,0.368569)$ \\
& 0.65352 & 0.073932 & 0.90481 & 0.059448 & $2.0914 \mathrm{e}-24$ & $(0.219731,0.282852)$ \\
& 0.64345 & 0.056831 & 0.98384 & 0.08896 & $2.9029 \mathrm{e}-27$ & $(0.304899,0.375884)$ \\
\hline \multirow{3}{*}{ Grey matter } & 0.31149 & 0.087768 & 1.2437 & 0.21282 & 0.0000 & $(0.943582,0.920757)$ \\
& 0.33376 & 0.10896 & 1.2126 & 0.26517 & 0.0000 & $(0.890516,0.867075)$ \\
& 0.41577 & 0.16546 & 1.2262 & 0.24268 & 0.0000 & $(0.822167,0.798673)$ \\
& 0.40791 & 0.26618 & 1.2125 & 0.28234 & 0.0000 & $(0.819749,0.789466)$ \\
\hline \multirow{5}{*}{ White matter } & 0.46905 & 0.048106 & 1.8965 & 0.090815 & 0.0000 & $(1.432445,1.422388)$ \\
& 0.48283 & 0.063785 & 1.8917 & 0.090622 & 0.0000 & $(1.414607,1.403110)$ \\
& 0.59634 & 0.06578 & 1.8224 & 0.06586 & 0.0000 & $(1.233771,1.218308)$ \\
\hline
\end{tabular}

Table 4.5: Mean values of single voxel $R_{1}$ fits, $\overline{R_{1}}$, and standard deviation, $\sigma$ obtained via Inversion recovery(IR) and saturation recovery(SR) for volunteer cohort. The results are separated by region, then ordered by volunteer; volunteer 1, volunteer 2 scan 1, volunteer 2 scan 2 , volunteer 3 .

\subsection{Discussion}

Comparing the $R_{1}$ relaxivity values obtained for our $\mathrm{MnCl}_{2}$ solutions with those reported in the literature, we find that the $R_{1}$ relaxivity of $6.397 \mathrm{mM}^{-1} \mathrm{~s}^{-1}$ for $\mathrm{MnCl}_{2}$ solutions reported in [55] fall within the $95 \%$ confidence interval of the $R_{1}$ estimate obtained with our IR protocol. Specifically, our value obtained with the IR protocol is about $4 \%$ lower than the value reported in [55], while SR relaxivity is almost $32 \%$ higher, and the discrepancies are even greater for the VFA method. Even accounting for the fact that most of the scans were done for gel phantoms — which have a higher relaxivity than the solutions — the IR results suggest that the relaxivity of the $1 \%$ agar gels is approximately $16 \%$ higher than the value for the solutions, but the relaxivity obtained for $\mathrm{MnCl}_{2}$ gels with the two-point VFA method 
$(2,15)$ is approximately $67 \%$ higher than the value reported in [55]. While this may suggest that the IR method is the most accurate, the study by Thangeval et al. was performed using a similar IR protocol and a $3 \mathrm{~T}$ Siemens scanner similar to ours, and we have found excellent consistency in our phantom data when the same protocol is repeated with the same phantoms on the same hardware.

Although each method of $R_{1}$ mapping was able to discriminate between the different contrast phantoms, the spread and overlap of the distributions obtained for a single phantom varied depending on the method used. More importantly, the large difference in the means and distributions of the $R_{1}$ values for same phantom or tissue ROI, obtained with different methods, suggests that $R_{1}$ maps acquired with different methods can not be reliably compared. Furthermore, it is observed that the variation of $R_{1}$ values - for a particular region of the brain - as a result of acquisition method is in fact larger than the variation from region to region. This suggests that differences in the $R_{1}$ quantification method may be a major factor in the large variability of the values reported in the literature.

It could also be argued that it does not matter if we measure the true $R_{1}$, or even if there is a true $R_{1}$, provided that we have a protocol that is effective at discriminating different tissue types, and gives results that are consistent and reproducible with a small margin of error. However, this is problematic for quantitative MRI and quantification of biomarkers, as the characteristics of a true biomarker should not be dependent on the MRI protocol or hardware characteristics, aside from physical variables such as field strength.

The discrepancies in the $R_{1}$ values obtained using VFA methods in particular have implications for many clinical applications. For instance in DCE, $R_{1}$ values obtained using a two-point (typically 2,15) VFA method are often combined with known relaxivities of the contrast agent to model contrast agent uptake curves, e.g., using the Tofts model. Given known relaxivities of free Gadolinium within a particular tissue, $R_{1}$ mapping has the potential to provide absolute Gadolinium quantification for these regions.

The findings of this study indicate that the choice of acquisition method affects not only $R_{1}$, but also the relaxivity. Relaxivity for the gel phantoms was found to vary between 7.16 $(5.64,8.69)$ and $11.91(9.40,14.45) \mathrm{s}^{-1} \mathrm{mmol}^{-1}$ for inversion recovery and 12-point VFA respectively. This suggests that ideally, the uptake model should be chosen to match the 
desired $R_{1}$ mapping method, so as to reduce misidentification of the contrast agent uptake curves and free Gadolinium deposits. In practice, accuracy and precision must be balanced with the efficiency of the acquisition protocol. While VFA methods are more susceptible to $B_{0}$ and $B_{1}$ inhomogeneities than the recovery methods, the drastic reduction in acquisition time makes them the far more practical choice for methods such as DCE.

The review by Bojorquez et al. found $R_{1}$ for grey matter was reported between 0.55 , and $1.03 \mathrm{~s}^{-1}$. The in-vivo $R_{1}$ values from IR were found to lie within this same range, however, the SR were found to be lower. For white matter a range of 0.70 to $1.33 \mathrm{~s}^{-1}$ was reported in the review, whereas the white matter $R_{1}$ for this volunteer group were found to lie above and below this range for IR and SR respectively. The large disparity between the IR and SR values is a consistent finding of this study. For all regions and volunteers, the SR and IR methods fell outside the bounds of standard deviation, with t-test p-values ranging from 0 to $10^{-10}$. IR $R_{1}$ values are found to be consistently higher than SR for the same regions, in agreement with the findings of the phantom study. SR methods show poor reproducibility in grey and white matter quantification, with inter-participant variation on the same order as intra-participant variation. Conversely, the IR methods display consistent intra-participant results, while maintaining inter-participant variation.

If different methods to determine $R_{1}$ produce such different results, this raises the question what we are really measuring. Is there a true $R_{1}$ value that depends only on the characteristics of the phantom or tissue examined, and if so what protocol gives the most accurate results for a wide range of tissues?

\subsection{Conclusion}

While the temporal consistency of phantom data, and in-vivo IR maps is promising, the marked separation of IR and SR maps is troubling, and emphasises the need for standardisation of protocols where possible. Comparisons of $R_{1}$ measurements across acquisition protocols should be done tentatively, if at all, to avoid invalid conclusions. This is of particular relevance to DCE scans, where two sources of contrast agent relaxivity are compared. Our results suggest that this is not best practice, and that it might be preferable to use a single sequence for both measurements of $R_{1}$. In this particular study, variable flip angle 
measurements were limited to phantom data, and the affect of the combinations of flip angle were not explored fully. Other sequences, such as Look-Locker etc., could also have been considered, given more time. To expand our findings, and further elucidate the protocol dependence of $R_{1}$, a multi site and field strength study is likely required. Expansion of the variable flip angle study, and the inclusion of more methods, will provide a more rigorous investigation of this phenomenon. 


\section{Quantification of edited magnetic resonance spec- troscopy: a comparative phantom based study of analysis methods.}

\section{$5.1 \quad$ Introduction}

Magnetic resonance spectroscopy (MRS) is an analytical branch of MR which produces spectra, rather than images. An in-vivo MR spectrum is composite signal containing the frequency components of all observable spin systems. Through processing and analysis it is possible to derive molecular concentration information from the spectra, allowing a snapshot of the metabolic processes that are occurring. Fig. 5.1 shows an MR spectrum of a human brain, acquired from a volunteer at Swansea University. Molecules within the brain may be identified by their characteristic peaks, and even quantified relative to the others in the spectrum. As such, MRS is a powerful diagnostic tool [79], providing complimentary data to the structure-based imaging of MRI. MRS provides a non-invasive means to assess the chemical composition of anatomy, invaluable for sensitive areas of the body, where biopsy would be problematic. While the brain is the most common application, there are other regions where it has benefit e.g. assessment of cancer of the prostate $[80,81]$ and breasts $[82,83]$, or quantification of fatty liver disease $[84,85]$. MRS can also be utilised in the diagnosis and treatment planning of cancers; it allows identification and staging of tumours [86, 87], delineation of tumour boundaries to a higher precision than MRI [88], and the differentiation of cancer type [89].

The focus of this chapter is the detection and quantification of some the major MRS signal generating molecules in the brain. Using basic spectroscopic sequences at $1.5 / 3$ T, several metabolites are identifiable. Depending on the choice of acquisition parameters, these include choline (Cho), creatine (Cr), and N-acetylaspartate (NAA), glutamate (Glu), glutamine (Gln), myo-inositol (Myi), lactate (Lac), alanine (Ala), as well as lipids and macromolecular resonances. Short $T_{E}$ sequences will better detect short $T_{2}$ molecules, meaning sequence parameters will change depending on intended target. NAA is one of the most abundant amino acids in the central nervous system, and produces the largest 


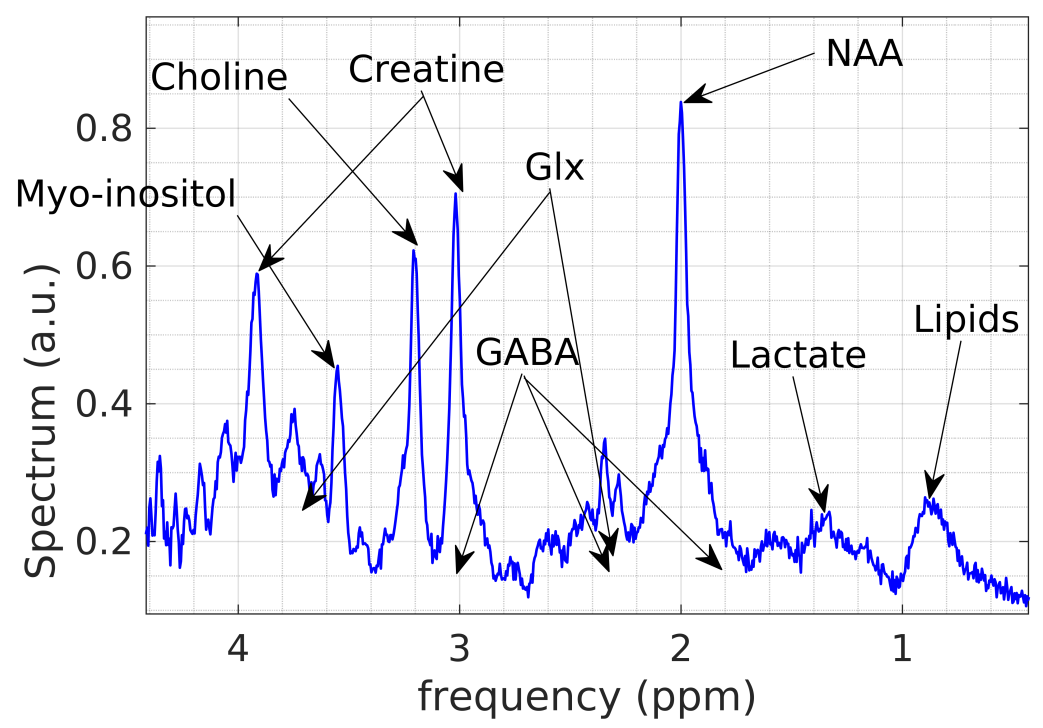

Figure 5.1: STEAM spectrum of a human brain. Labels indicate the locations of characteristic peaks. Overlap with larger peaks makes the detection of lower concentration signals problematic. Frequencies are plotted right to left, by convention.

signal in the brain, as can be seen in Fig. 5.1. It produces a single peak at 2PPM, with N-acetylaspartylglutamate (NAAG) at 2.04. Glu and Gln, are a pair of amino acids with very similar peaks, that are indiscernible with basic spectroscopy sequences, and are often identified as one combined peak shape, Glx. Cr, a nitrogenous organic acid, is another abundant signal generator, $\mathrm{Cr}$ produces a peak 3PPM, however this is again a composite peak of $\mathrm{Cr}$ and phosphocreatine $(\mathrm{PCr})$; a second resonance can be observed at 3.91PPM. A less abundant, but no less important molecule, is GABA. Gamma-Aminobutyric acid, or GABA, is the primary inhibitory neurotransmitter in the central nervous system, maintaining the excitation-inhibition balance [90]. Its prominent role in both neurotransmission and metabolism has led to extensive study, and a plethora of applications for its detection. GABA has been observed to exhibit reduced uptake in Schizophrenic patients [91], GABA receptor dysfunction has been observed in epilepsy sufferers, and GABA related processes have effects in the diabetic patients [92], and autism spectrum disorders [93]. GABA is also the subject of intense study by the psychological community, with GABA processes influencing impulsivity [94,95], drug addiction [96], anxiety disorders [30,97], and depression [98]. 
However, despite its wide range of applications, there are many challenges in its detection. GABA exists in the brain in mMolar concentrations, and relatively stronger signals, such as NAA and Cr, will shroud those of GABA (Fig. 5.1). It is only through the use of edited spectroscopy [25,99-101], or other advanced techniques such as multi-dimensional spectroscopy, making use of a larger parameter space $[29,30]$ that GABA becomes observable. The goal of this study is to assess the robustness of GABA quantification using a calibrated phantom study, and to compare some of the main analysis methods used for the quantification of GABA edited spectroscopy. This work is intended as a precursor study for the development of RF pulses for new techniques, Sec. 6, and new spectral decomposition methods, work being developed in conjunction with Max Chandler, and Frank Langbein of Cardiff University, Computer Science Department.

\subsection{Theory}

\subsubsection{Spectral processing techniques}

zero filling The FID of some sample is a signal of finite length, sampled at some discrete rate. The resulting number of points in the FID will determine the spectral resolution of any frequency domain spectrum. A relatively coarse sampling can be overcome using a process known as zero filling. Zero filling is a signal processing technique that can increase the digital resolution of a Fourier transformed spectrum. By appending the FID with additional zero-

valued components, the Fourier transform resolution can be increased accordingly. Zero filling is comparable to an interpolation process which can increase SNR by a factor of $\sqrt{2}[102]$. However, the process does not introduce any new information to the FID, so results are still limited by the information content of the FID. Over-filling can also add additional noise to the frequency domain spectra, further complicating the process of quantification.

Apodisation Another nuance of the Fourier transform becomes apparent when the start and end points of the time-domain signal do not align. The Fourier transform interprets this discrepancy as additional frequency components of the signal, introducing peaks to the frequency-domain that should not be present. This process is independent of experimental 
procedure, and is still present in perfect sinusoidal composite signals. If the end point is chosen to be a non-zero value, there will be additional peaks introduced in the frequencydomain. This can be overcome using a process known as apodization. If we "window" the time-domain signal, such that the end point is reduced to zero, we can eliminate these additional peaks. This process can also be used to reduce noise in a spectrum. A standard FID will usually require acquisitions on the order of seconds, by which point much, or all of the signal will have decayed. After some time, the FID will no longer be enhancing the signal peaks, but will only be adding to the noise of the spectrum. There are several options in windowing, the simplest being a cut-off window, that involves selecting a point in the FID - usually just after the major signal components have decayed — and then setting all subsequent points to zero. This reduces the noise components, but will still contain Fourier transform artefact peaks. Another, perhaps more commonly used example, is an exponential window:

$$
\omega(t)=e^{-t / T_{W}},
$$

where $T_{W}$ is a weighting time-constant. When the FID is multiplied by an exponentially decaying function, it reduces the weighting of the later points of the FID to zero. It also provides a larger weighting to earlier points in the FID, resulting in an enhanced SNR. However, when Fourier transformed, the resulting peaks will appear broader than in the original spectrum, an effect known as artificial line broadening. The Hamming window is another option, offering similar benefits. This function is given by:

$$
\omega(t)=0.54-0.46 \cos \left(2 \pi \frac{t}{L-1}\right), \quad 0 \leq t \leq L-1,
$$

where $L$ is the length of the window. There are also Hanning, Gaussian, and sinusoidal windows, all of which can reduce noise, improve low SNR data, and remove Fourier transform peaks, at the expense of peak broadening and arbitrary window length.

Singular-value decomposition The singular-value decomposition (SVD) is another method that can be employed to filter spectra. The SVD is a generalisation of the eigenvalue decomposition, a method of decomposing certain classes of matrices and representing them in terms of their eigenvalues and eigenvectors. We can write a matrix, $A$, in terms of a singular 
value, $\sigma$, and singular vectors, $u$ and $v$

$$
\begin{gathered}
A v=\sigma u, \\
A^{\dagger} u=\sigma v .
\end{gathered}
$$

Singular values are essentially a generalisation of eigenvalues. If our matrix, $A$ has dimensions $n \times m$, we can define an $n \times n$ matrix $S=A^{\dagger} A$. The singular values of $A$ can be found by taking the square root of the eigenvalues of $S$. If $A$ is a square symmetric matrix with positive, real elements, the singular values will in fact coincide with its eigenvalues. If the singular values of $A$ are compiled on the diagonal of an otherwise null matrix, $\Sigma$, we find:

$$
\begin{aligned}
& A V=U \Sigma, \\
& A^{\dagger} U=V \Sigma .
\end{aligned}
$$

Eq. (5.5) can then give us the singular-value decomposition equation:

$$
A=U \Sigma V^{\dagger}
$$

where $U$ and $V$ are square matrices, and $\Sigma$ has the same dimensions as our general matrix, A. Applying this to spectroscopy requires an additional step, the introduction of a Hankel matrix in place of $A$. The Hankel matrix, $H$, is a symmetric, square matrix which is constant across its skew-diagonals:

$$
H=\left[\begin{array}{ccccc}
H_{1} & H_{2} & H_{3} & H_{4} & \ldots \\
H_{2} & H_{3} & H_{4} & H_{5} & \ldots \\
H_{3} & H_{4} & H_{5} & H_{6} & \ldots \\
H_{4} & H_{5} & H_{6} & H_{7} & \ldots \\
\vdots & \vdots & \vdots & \vdots & \ddots
\end{array}\right]
$$

where, for spectroscopy, the $H_{N}$ are the $N$ elements of the free induction decay. The singular values, $S_{v}$, are acquired from the diagonal of the decomposed matrix, $\Sigma$. Fig. 5.2 shows the decomposition for an NAA, GABA, creatine phantom. Ordering the singular values by amplitude and plotting reveals the disparity between the components. The spectrum is composed of a few high amplitude components, which correspond to the major peaks in 


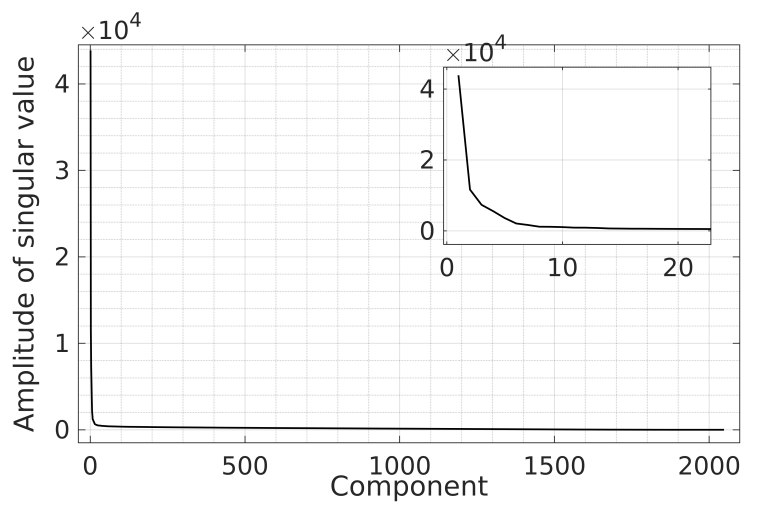

(a) Singular values ordered by magnitude.

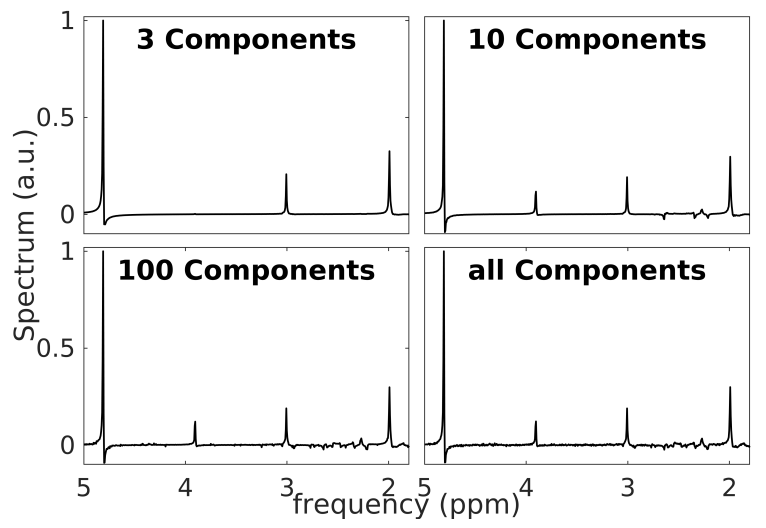

(b) Spectra reulting from specific filter length.

Figure 5.2: Singular value decomposition of an NAA/GABA/Cr phantom PRESS spectrum. In (a), singular values are ordered by amplitude and plotted, with singular values beyond 10 converging to zero. This reflected in the spectra in (b), with noise being added after the initial 10 components.

the spectrum. Including the first three components, for example, adds the water and NAA peaks, as well as one of the creatine peaks, with subsequent components making successively smaller contributions. Excluding singular values beyond a suitable threshold will remove noise from the data, improving SNR. Unlike previous filtering methods, the SVD will not broaden the peaks of the spectrum. However, its effectiveness at low SNR is dubious, as peaks that are too close to the noise floor will be erroneously excluded, skewing the resulting data.

Baseline subtraction Analysis of MR spectra may be further complicated by the presence of a signal baseline; a background signal which interferes with the peaks of interest. A uniform baseline, across the entire spectrum, can be easily removed by a simple subtraction, however, many baseline signals will have a frequency dependent component. MRS in water based media relies heavily on the suppression of water protons to be able to identify the other signals. However, water suppression may not remove the entirety of this signal, and a residual water baseline will be observed. This will introduce a frequency dependent modulation of the signal, with peaks close to the water peak being artificially augmented when quantified. 
Residual water subtraction is typically performed by a variation of the SVD method, the Hankel Lanczos singular-value decomposition [103]. Another potential source of baseline, in-vivo, are macromolecules and lipids, which can introduce a broad frequency dependent baseline $[104,105]$. This is often remedied by performing a linear, or polynomial fit on the signal floor of the data, which is then subtracted.

Frequency and phase correction Random noise will perturb any single FID, however, over multiple acquisitions these fluctuations will average out, and a single high quality spectrum can be obtained through the sum of individual FIDs. However, over the course of multiple acquisitions, it is possible to observe changes that are not relevant for a single scan. The temperature of gradient/RF coils, or that of the sample itself, can increase as a result of the scans, causing a change in the reference frequency of the acquired FID. A similar effect is observed in-vivo resulting from patient movement. The individual FIDs will be unchanged - barring some peak broadening, perhaps - but the shift of the centre frequency will manifest itself as a misalignment of the FIDs in the composite spectrum, resulting in broader peaks, and a lower SNR. A similar issue is observed in the phase of a spectrum, with the initial phase offset of a single acquisition unknown, a correction is required to avoid SNR reduction, and peak broadening. Zero-order phasing adjusts a single spectral line, with frequency dependent phase shift tackled by linear or first order phasing. The relationship between frequency and phase means the two issues are often linked, and corrected simultaneously for example, frequency and phase correction using residual water reference [106], frequency and phase correction using spectral registration [107], and residual water peak alignment, creatine fitting, and spectral registration [108].

\subsubsection{Quantification of spectra}

Spectral line shapes contain not only information of the molecule structure, but also relative concentration. Signals that are modulated by choice of $T_{E}$, frequency, phase, baseline, and noise can be corrected as discussed above, with remaining signal being indicative of concentration. The signal, $S_{M}$ produced by $N_{S}$ scans of a molecule of molar concentration, $M$, 
within a volume, $V$, is given by:

$$
S_{M}=N_{S} \times G \times \omega_{0} \times M \times V \times f_{\text {seq }} \times f_{\text {coil }},
$$

where $R$ is the receiver gain, $\omega_{0}$ is the Larmor frequency, and $f_{\text {seq }}$ and $f_{\text {coil }}$ are complex functions describing modulations due to sequence and coil respectively [12]. Some parameters in this equation are unknown, for example, the signal modulation due the coils; direct quantification of an MR signal is not possible. However, it is possible to calculate $M$ relative to some reference compound of known concentration, $R$;

$$
M=R \frac{S_{M}}{S_{R}} C_{M R}
$$

where, $S_{M}$ and $S_{R}$ are the detected signals of the compound of interest and reference, respectively, and $C_{M R}$ is a correction factor to account for differences in relaxation properties, $\gamma$, spatial variation etc. Calculation of $C_{M R}$ is often time consuming and impractical, so it is far more common to simply report a concentration ratio, $\frac{S_{M}}{S_{R}}$. Referencing can be performed in one of two ways, externally, or internally. External referencing involves placement of a phantom containing a known concentration of some reference compound, within the RF sensitive region [109]. The reference solution must produce a stable, well resolved resonance that does not interfere with the peaks being investigated. Care must be taken when using this approach; phantom placement has a direct impact on RF homogeneity, and coil loading, both of which can impact the observed signal. Internal referencing involves taking peak ratios relative to one peak of the given spectrum. The peak chosen should be a well resolved, strong signal that is stable with respect to time and environment. In-vivo, peak amplitudes are commonly reported as a ratio of creatine or NAA, due to their relatively stable concentrations. The water signal may also be used as a reference compound [110], using estimations of water densities of grey and white matter, for example, to estimate the concentration in the voxel of interest. The relative impracticality of the external approach makes internal referencing the far more common choice. Quantification of the signals themselves can be done in a number of ways.

Numerical area fitting The area of a particular resonance should scale linearly with the concentration of the molecule that produced it, with the height of its constituent peaks 
scaling with the number of protons at that resonance. The simplest way to quantify this area is numerical integration. An MRS spectrum is a discrete function, so is accurately quantified by integral approximations such as the trapezium rule. However, small variations, such as noise and baseline, will also be captured by this methods, so it can be preferable to fit peaks with a Lorentzian distribution function. Fitting a single Lorentzian is only feasible in the case of an individual, resolved peak, so has limited applicability. A more general Lorentzian fitting technique can be used in conjunction with a least squares optimisation.

Least-squares fitting It is possible to model an FID consisting of $n$ signal values, $y_{n}$, as a sum of exponentially damped, complex-valued sinusoids, sampled at uniformly distributed times $t_{n}=(n+\delta) \Delta t, n=0: N-1$ :

$$
y_{n}=\sum_{k=1}^{K} c_{k} z_{k}^{n+\delta}=\sum_{k=1}^{K} a_{k} e^{i\left(\phi_{0}+\phi\right)} e^{\left(-d_{k}+2 i \pi f_{k}\right) t_{n}},
$$

where $K$ is the model order, $f_{k}$ the frequencies, $a_{k}$ the amplitudes, $d_{K}$ the damping factors, $\phi_{0}$ the zero-order phase, and $\phi_{k}$ the individual phase adjustment [103]. A least-squares minimisation algorithm is performed, the specific implementation of which will vary, to find the optimal set of parameters that model the spectrum, a process which is usually independent of user interaction. Lorentzian components with a high amplitude, $a_{k}$, contribute more significant peaks to the spectrum, and may be correlated to the constituent molecule. Smaller amplitudes can similarly be regarded as noise, and discarded. There are limitations to this method, especially for low SNR data. Prior knowledge, and user interaction is not commonly implemented, so high levels of noise restrict the accuracy of the decomposition of the spectrum.

Basis set fitting Basis set fitting methods take a similar approach to least-sqaures fitting, but differ in the choice of model function. Instead of representing the spectrum as an arbitrary array of Lorentzian functions, a linear combination of a set of basis functions is used. The basis - a set of spectra, one for each anticipated constituent molecule can be generated using calibrated phantom spectra, or via quantum mechanical simulated data. This approach reduces the amount of misidentified noise, improves the resolution of 
interfering signals, and improves identification of molecules generating multiple resonances. Some metabolites have a signal which depends upon the molecular environment, for example NAA's PH dependence (Fig.5.6), or temperature based frequency shifts, Fig. 5.5, which can actually be used to quantify temperature $[16,111]$. The basis set fitting methods are unable to account for such environmental changes, which leads to misidentification of metabolite signals.

\subsection{Materials and Methods}

\subsubsection{Phantom preparation}

The phantom study consists of four experiment series - two solution, and two gel — where the GABA concentration is varied over multiple acquisitions of a fixed protocol. The two solution series were prepared by dissolving the required concentrations of metabolites in $290 \mathrm{ml}$ of de-ionised water. Table. 5.3 show the various metabolite concentrations. The scan protocols were applied for each row in the table, with the solution adjusted between scans. To reduce the uncertainty resulting from low weight metabolite changes, finer GABA adjustments were made using a concentrated GABA solution, administered via a syringe. In the second solution, $\mathrm{pH}$ monitoring was introduced, with adjustments made after each metabolite change. These adjustments were made using a $36 \%$ hydrochloric acid solution, and a $<4 \%$ sodium hydroxide solution to maintain a $\mathrm{pH}$ of $7 \pm 0.3$.

For the gel series, a set of solutions were made in advance of scanning, along with a $1 \%$ concentration of agar. The solution was then heated to between $80^{\circ}$ and $90^{\circ}$ while stirred until the agar had dissolved. The solutions were allowed to cool over night in two hemispherical moulds, which were combined once the mixture had almost set.

\subsubsection{Scan protocols}

All scan protocols were conducted at Swansea University's Clinical Imaging Facility using a Siemens 3T Magnetom Skyra. The scanner room is temperature controlled to $20^{\circ} \mathrm{C} \pm$ $0.6^{\circ} \mathrm{C}$. All scans were conducted at this temperature, however, the GLX series was also heated to body temperature for a set of rescans to examine the effect of temperature shift in 


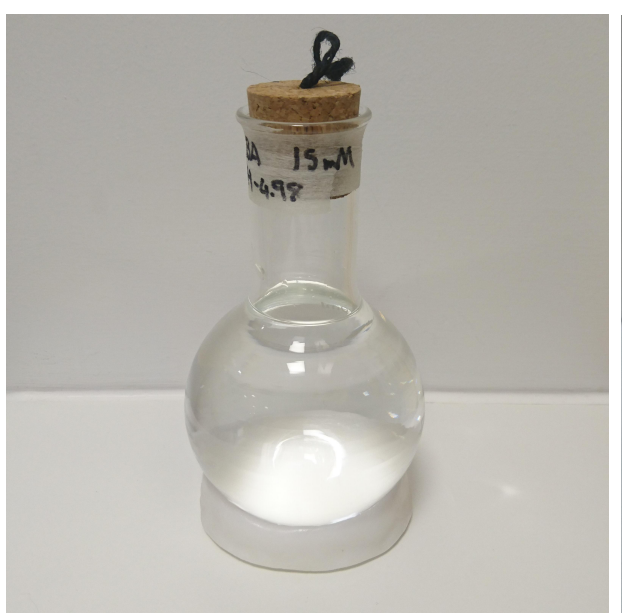

(a) Solution phantom

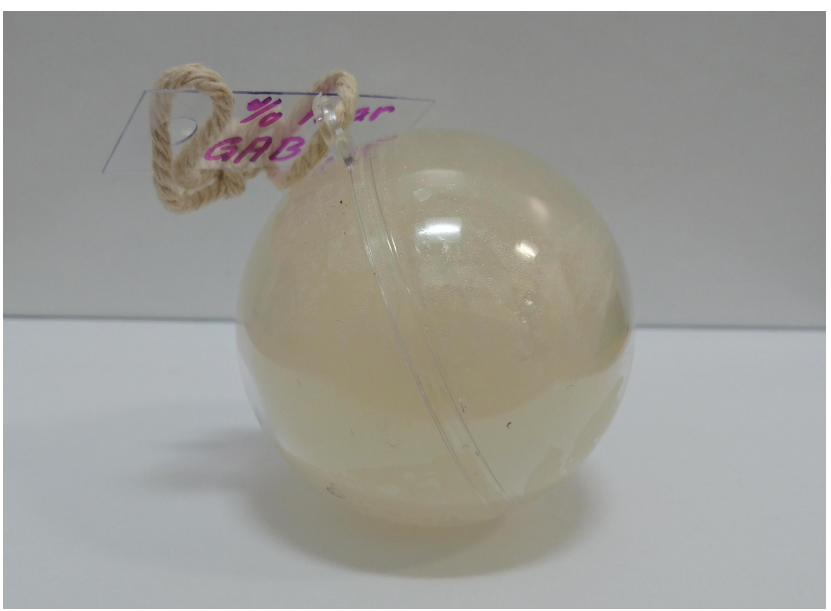

(b) Gel phantom

Figure 5.3: Images spherical solution and gel phantoms.

\begin{tabular}{c|ccccccccccccc} 
NAA & 15.0 & 15.0 & 15.0 & 15.0 & 15.0 & 15.0 & 15.0 & 15.0 & 15.0 & 15.0 & 15.0 & 15.0 & 15.0 \\
\hline $\mathrm{Cr}$ & 0.0 & 8.0 & 8.0 & 8.0 & 8.0 & 8.0 & 8.0 & 8.0 & 8.0 & 8.0 & 8.0 & 8.0 & 8.0 \\
\hline GABA & 0.0 & 0.0 & 0.5 & 1.0 & 1.5 & 2.0 & 2.5 & 3.0 & 4.0 & 6.0 & 8.0 & 10.0 & 11.6
\end{tabular}

Table 5.1: GABA1 - Solution series with fixed NAA and Cr concentration, and GABA varied. $\mathrm{pH}$ stabilisation is applied here, maintained to $7.0 \pm 0.2$.

\begin{tabular}{c|ccccccccccccccc} 
NAA & 15.0 & 15.0 & 15.0 & 15.0 & 15.0 & 15.0 & 15.0 & 15.0 & 15.0 & 15.0 & 15.0 & 15.0 & 15.0 & 15.0 & 15.0 \\
\hline $\mathrm{Cr}$ & 0.0 & 8.0 & 8.0 & 8.0 & 8.0 & 8.0 & 8.0 & 8.0 & 8.0 & 8.0 & 8.0 & 8.0 & 8.0 & 8.0 & 8.0 \\
\hline GABA & 0.0 & 0.0 & 0.5 & 1.0 & 1.5 & 2.0 & 2.5 & 3.0 & 4.0 & 5.0 & 6.0 & 7.0 & 8.0 & 9.0 & 12.0
\end{tabular}

Table 5.2: GABA2 - Solution series with fixed NAA and Cr concentration, and GABA varied. No $\mathrm{pH}$ stabilisation was applied, resulting in an acidic solution.

\begin{tabular}{c|cccccc} 
NAA & 15.0 & 15.0 & 15.0 & 15.0 & 15.0 & 15.0 \\
\hline $\mathrm{Cr}$ & 0.0 & 8.0 & 8.0 & 8.0 & 8.0 & 8.0 \\
\hline Glu & 12.0 & 12.0 & 12.0 & 12.0 & 12.0 & 12.0 \\
\hline Gln & 3.0 & 3.0 & 3.0 & 3.0 & 3.0 & 3.0 \\
\hline GABA & 0 & 1.5 & 3.0 & 5.0 & 7.0 & 10.0
\end{tabular}

Table 5.3: GLX - Gel series with fixed NAA, Cr, Glu, and Gln concentration, and GABA varied. $\mathrm{pH}$ stabilisation is applied here, maintained to be $7.0 \pm 0.2$. Here, agar is used as the gelling agent, at $1 \%$ concentration. 
the quantification procedure. Signal acquisition was done using the four channel spine coil element "SP2". The phantom was aligned with this element, then raised to isocentre using a custom phantom holder. This ensured the maximum field homogeneity for the scans. Double spin-echo interference field maps were acquired to assess the homogeneity of the phantom, then manual shimming was performed to optimise the spectral width. Double echo

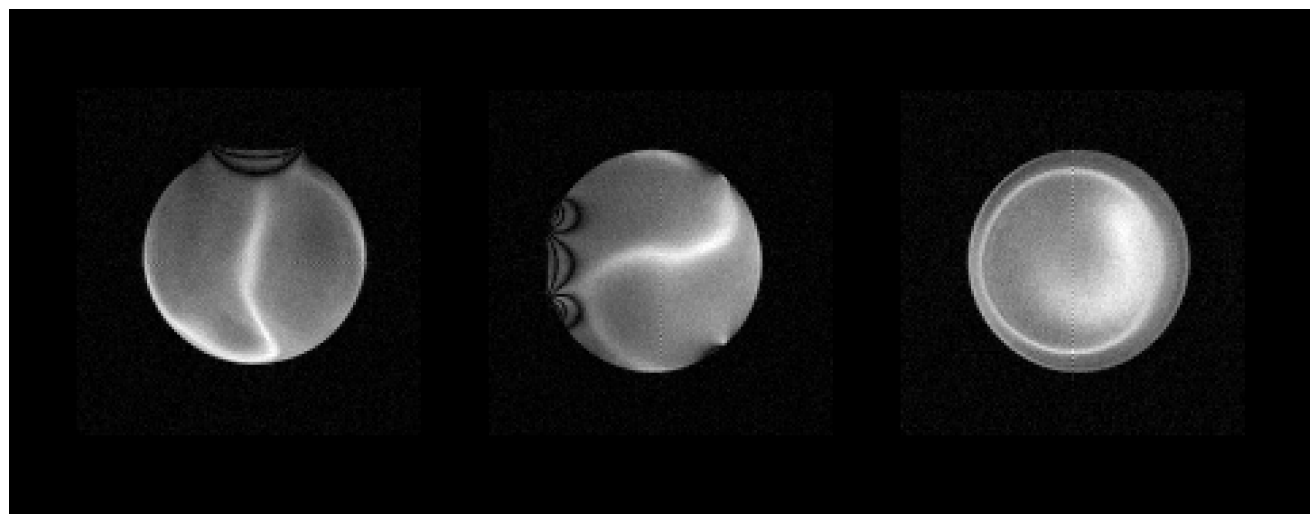

(a) $290 \mathrm{~mL}$ solution phantom.

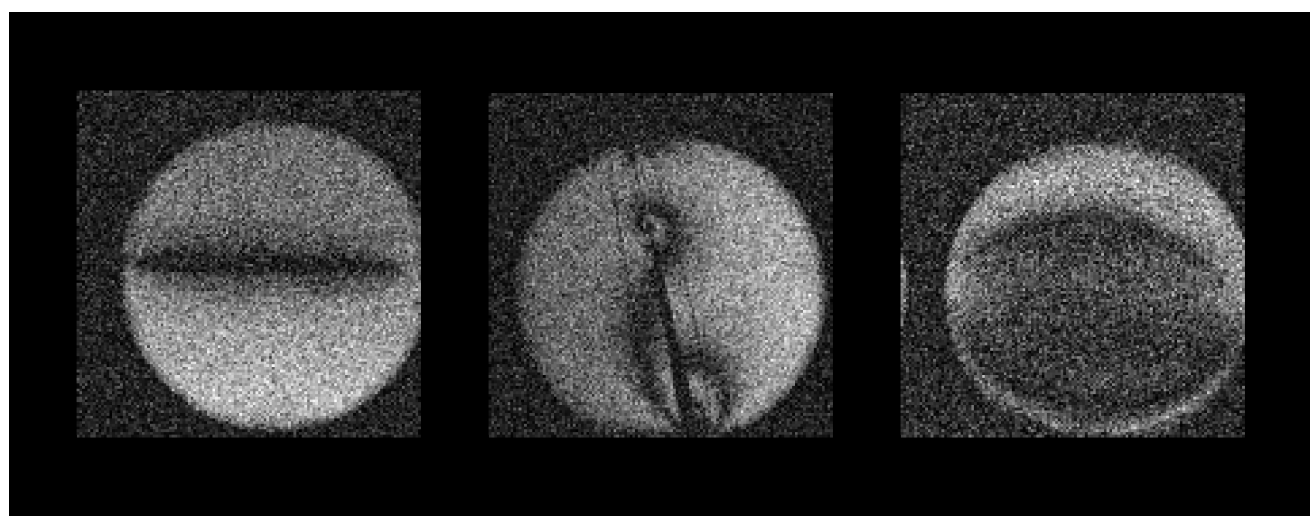

(b) $100 \mathrm{~mL}$ gel phantom.

Figure 5.4: Double spin echo $B_{0}$ maps for solution and gel phantoms. The concentration of fringes indicate homogeneity in the $B_{0}$ field, with large fringe spacing suggesting a homogeneous field.

interference field mapping is based on a double spin-echo sequence calibrated to maximize interference between the regular and stimulated echo pathways. The refocusing pulses are applied at times $\frac{1}{2} T_{E}$ and $\frac{3}{2} T_{E}$. At time $2 T_{E}$ both a stimulated, and regular echo are produced at readout. The phase difference between the two echoes is determined by the $T_{E}$, and is 
proportional to the precessional frequency difference, $\Delta f$, and the field inhomogeneity, $\Delta \phi$, is:

$$
\Delta \phi=2 \pi \Delta f T_{E}=\gamma \Delta B T_{E}
$$

Therefore the frequency difference between two fringes in the image is: $\Delta f=\frac{1}{T_{E}}$. Thus, if $T_{E}=20 \mathrm{~ms}$, then the frequency difference between two consecutive fringes is $50 \mathrm{~Hz}$, while for $T_{E}=100 \mathrm{~ms}$ the difference corresponds to $10 \mathrm{~Hz}$. From the frequency difference, we can easily obtain the $B_{0}$ field inhomogeneity in Tesla by multiplying with the Larmor constant, $\gamma$, of the nucleus. Double spin echo field mapping is useful to quickly visualize the pattern of the inhomogeneity. The field maps are shown in Fig. 5.4 were acquired using $T_{E}$ of $20 \mathrm{~ms}$. The homogeneity of the solution phantoms, and their larger volumes, allowed isocentre voxels to be selected in all cases, with inhomogeneous regions restricted to the neck of the flask. Gel phantoms were found to have higher levels of inhomogeneity in the centre, due to the gelling procedure used in this case. ROIs for the gels were selected outside of this region to preserve the quality of the data.

\subsubsection{Analysis methods}

There have been many analysis tools developed for magnetic resonance spectroscopy data, some of the major tools are presented here, along with their use within this study.

Tarquin Totally Automatic Robust Quantitation in NMR (TARQUIN) $[113,114]$ is a time domain basis set analysis tool. TARQUIN is a free to use software package complete with GUI. Quantification is performed using the Lawson-Hanson non-negative least-squares algorithm [115] to fit a basis set generated by its custom NMR simulator, or a user defined alternative. Residual water removal is performed using HSVD, and automatic phase and frequency correction is applied. TARQUIN does not perform a full MEGA-PRESS simulation, but rather models the expected signal, and adjusts its phase correction procedures to accommodate the negative NAA peak. Frequency calibration is made relative to NAA, and results are reported as fit amplitudes. 
JMRUI JMRUI $[116,117]$ is a free software package for the processing and analysis of MRS datasets. It was built upon its Matlab-based predecessor - magnetic resonance user interface (MRUI) - developing a JAVA based UI for its software. JMRUI is a comprehensive package which includes a variety of processing and quantification techniques. JMRUI utilises several variations of singular value decomposition for processing and quantification, including Hankel singular-value decomposition (HSVD) [118, 119], Hankel Lanczos singularvalue decomposition (HLSVD) [103], Hankel total least squares (HTLS) [120], linear prediction and singular-value decomposition method (LPSVD) [121]. JMRUI also includes "Advanced method for accurate, robust, and efficient spectral fitting" (AMARES) [122] which expands on a previous algorithm VARPRO [123]; a time-domain non-linear least-squares method. AMARES uses the dn2gb algorithm [124] which improves on the performance Levenberg-Marquardt algorithm of VARPRO under certain conditions [122]. AMARES also changes its approach to prior knowledge imposition; implementing a singlet fitting, rather than multiplet. JMRUI also has two basis set quantification algorithms that make use of its NMR simulation package, NMRSCOPE-B $[125,126]$. NMRSCOPE-B is a quantum mechanical simulation tool that can simulate metabolic, and arbitrary user-defined models under influence of a variety of common pulse sequences. The first, "quantitation based on semi-parametric quantum estimation" (QUEST) [127,128], is a time-domain quantification tool which is based on AMARES and VARPRO, and uses the Levenberg-Marquardt algorithm. QUEST includes a semi-parametric approach to handle spurious signal resulting from macro molecules and lipids. "Automated Quantitation of Short Echo time MRS spectra" (AQSES) [129] is the second, a time domain basis set quantification package. AQSES uses a modified VARPRO algorithm [130], with introduces the imposition of prior knowledge in the form of upper and lower bounds on the nonlinear parameters. Macromolecular baselines are fitted non-parametrically using penalized splines.

For this study, the QUEST and AQSES algorithms were used to quantify the spectra. A single MEGA-PRESS pulse sequence was defined in NMRSCOPE-B, with sequence parameters adapted to those of our experimental data. Two basis sets were generated, one for the GABA/Cr/NAA, and one for the GABA/Cr/NAA/GLX. Manual frequency calibration was performed, using the NAA peak as a reference. Results reported are the fit amplitudes 
resulting from this process.

LCModel LCModel [131,132] is a Linux based black box MRS analysis tool. LCModel can be used with its internal in-vitro basis, or with any arbitrary basis set specified by the user. The baseline signal, resulting from macromolecules, is established using spline fits. Fitting is attempted using the Gauss-Newton non-linear least-squares algorithm, with a Marquardt modification [133]. For this study, LCModel analysis was performed in Cardiff university by Max Chandler due to the licensing restrictions of LCModel. The analysis was performed with two user basis sets, one with the Govindaraju GABA model, and one with the Kaiser et al. model. The details of the models are found in Appendix. C . Both sets of results are reported in terms of their fit amplitudes.

VeSPA Versatile Simulation, Pulses and Analysis package (VeSPA) [134] is a python based amalgamation and extension of three software packages, MatPulse [135], GAVA [136], and SITools [137]. VeSPA utilises the Voigt method — based on the papers by Young, Soher, et al. $[138,139]$ - an automated spectral analysis procedure that combines a parametric model of metabolites with a non-parametric characterization of the unknown signal components. The basis set is generated using the Python implementation of the GAMMA simulator; PyGAMMA [140], and baseline estimates made using the residual signal. VeSPA is excluded from this study, as their current MEGA-PRESS simulator is still under refinement.

GANNET GABA-MRS Analysis Tool (GANNET) [141] is a black box Matlab based analysis tool, specifically designed to process GABA MEGA-PRESS data. GANNET consists of two separate modules, GannetLoad, and GannetFit. The first module receives time-domain data (and water un-suppressed spectra if available), performs channel combination, adds line broadening, frequency and phase corrections, outlier rejection, and time averaging. This structure can then be analysed independent of the fitting module. The fitting is performed using non-linear least-squares algorithms (lsqcurvefit and nlinfi). It uses a five parameter Gaussian model to estimate the 3PPM GABA signal in the difference spectrum, a six parameter Lorentzian model to estimate the 3PPM Creatine signal in the off spectrum, and if available, a six parameter Gaussian-Lorentzian model to fit the unsuppressed water sig- 
nal. Quantitative results are then reported as integral ratios of GABA to Creatine, and a concentration relative to water, NAA, or GLX.

In-house analysis As well as the popular tools, the data was analysed with in-house software, written in Matlab. Zero filling was applied to four times the vector length. Frequency corrections used the 2PPM NAA peak as a reference, and fitting was applied. Numerical integration, was applied within intervals, $\nu_{G A B A}=(2.88,3.14), \nu_{N A A}=(1.9,2.1), \nu_{G L X 1}$ $=(3.66,3.81)$, and $\nu_{G L X 2}=(2.27,2.44)$. This process was selected based on the analysis of smaller preliminary experiments. Results suggested good performance, even compared to the established tools, so this fit procedure is included as a baseline for the experiment. The results of this analysis are reported as peak area ratios.

\subsubsection{Calibration}

As stated previously, temperature has a direct effect on MR spectra, particularly the water peak. The analysis tools we investigated are calibrated for in-vivo data, at body temperature. Our study is a room temperature phantom study, so care must be taken in frequency calibration. To further elucidate the temperature dependence of the water peak, we performed a calibration experiment using a water/DSS phantom. DSS is stable with respect to temperature and $\mathrm{pH}$, so is often used as a reference peak in NMR studies. A round bottom flask was filled with $290 \mathrm{~mL}$ of deionized water, and $1 \mathrm{mmol}$ of DSS dissolved within, and the $\mathrm{pH}$ recorded at 6.97 . The flask was heated in a water bath to $40^{\circ} \mathrm{C}$, and placed in the scanner, mirroring the set-up of the phantom series. A series of PRESS spectra were acquired, with the temperature measured intermittently. The maxima of the water and DSS were quantified, and the separation of the peaks recorded. Fig. 5.5 shows this separation plotted against the mean temperature during the scan. The mean was calculated based on the temperature recorded before and after the scan. Error in the maximum was estimated based on the frequency resolution, while error in the temperature taken to be the separation of the readings. The DSS-water peak separation was found to vary between 4.80, and 4.64 $\mathrm{PPM}$, with a negative linear gradient of $-9.676 \times 10^{-3} \mathrm{PPM} /{ }^{\circ} \mathrm{C}$ with $95 \% \mathrm{CI}$ of $(-10.11$, $-9.246) \times 10^{-3} \mathrm{PPM} /{ }^{\circ} \mathrm{C}$, and $R^{2}$ of 0.9910 . With water used as the centre frequency for the 


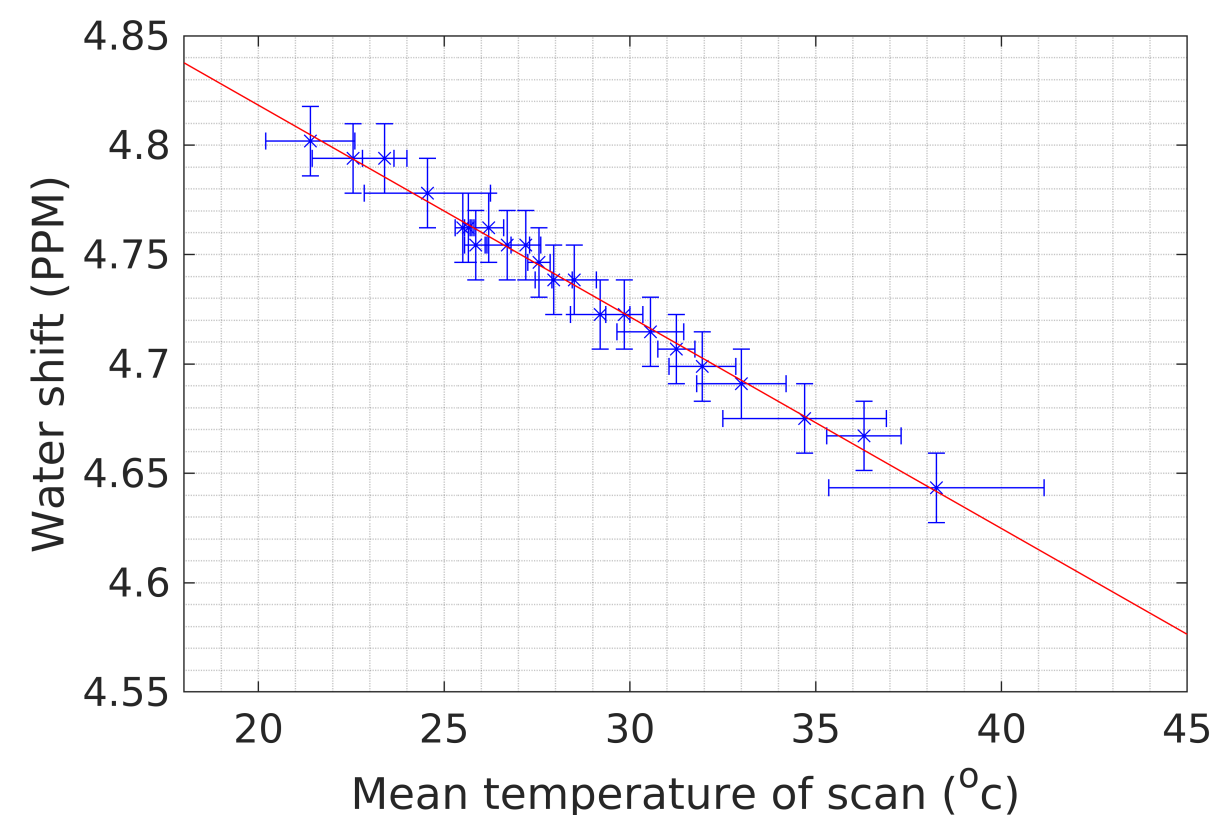

Figure 5.5: The DSS peak is stable with respect to both $\mathrm{pH}$ and temperature, so any change in the separation between the water and DSS peaks is a result of the water peak shifting.

spectrometer, this shift of approximately 0.164 PPM from body to room temperature, will be present in the peaks of all other metabolites. This is accounted for by referencing all scans to NAA, aligning the large NAA singlet to its correct position of 2.00 PPM.

The NAA/Cr/GABA series was performed twice, once with $\mathrm{pH}$ calibration, and once without. NAA is natively acidic, with a $\mathrm{pH}$ dependent spectrum. We postulated that basis set quantification would fail for phantoms outside the ph range expected in the healthy brain. Fig. 5.6 shows difference pH makes to the NAA spectrum. While the 2PPM NAA peak remains stable, the secondary peaks are unrecognisable, a challenge for basis set methods. MEGA-PRESS simulations were performed using the FID-appliance (FID-A) [142], a simulation and data processing package for MRS. MEGA-PRESS difference spectra were generated to mirror the sequence parameters of the phantom study, with finite-bandwidth editing pulse alternating at $1.9 \mathrm{ppm}$ and $7.4 \mathrm{ppm}$, at a field strength of $2.89 \mathrm{~T}$, bandwidth of $1250 \mathrm{~Hz}$, with two and four step phase cycling, and ideal refocusing pulses. Spectra were generated for creatine, NAA, Glutamine, Glutamate, shown in Fig. 5.7, three of the most commonly used GABA models, shown in Fig. 5.8. The difference spectra were then com- 


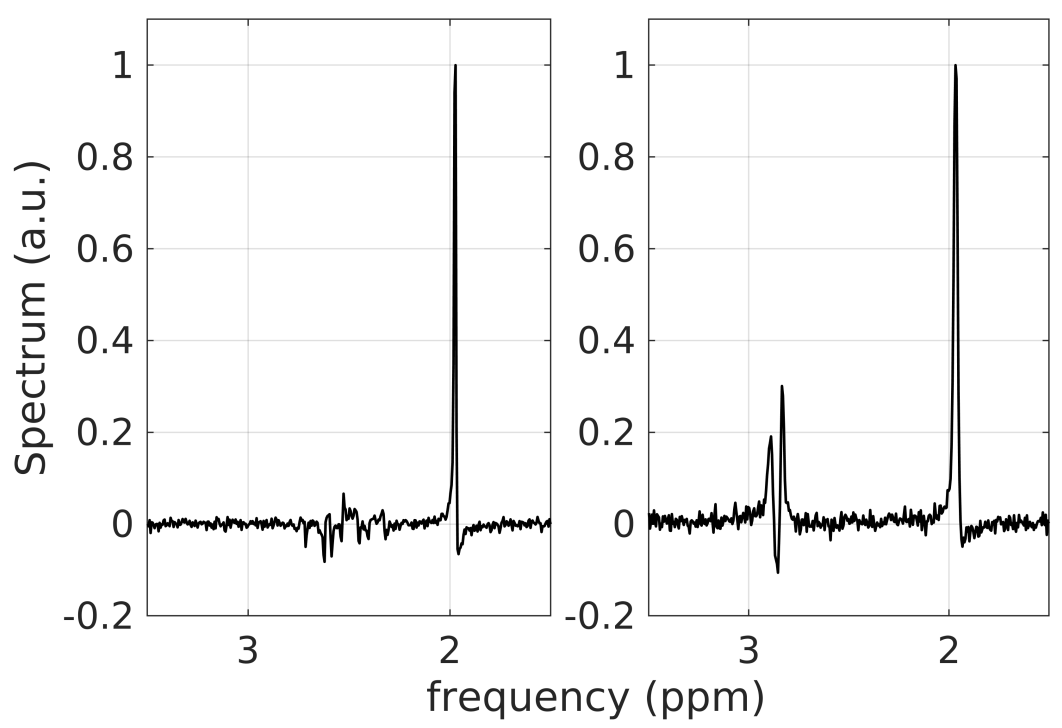

Figure 5.6: NAA phantom spectra for neutral, and acidic pH, respectively. The main 2PPM singlet remains stable, but the secondary peaks change.

bined to reflect the ratios of the phantom experiment, with the GABA concentration varied between $1 \mathrm{mmol}$ and $12 \mathrm{mmol}$ at $1 \mathrm{mmol}$ intervals, for both models. The combined spectra were analysed with a peak integration, with the resulting four series shown in Appendix. D. The GABA to NAA peak area ratio was plotted against the GABA concentration for the GABA/Cr/NAA series, and the GABA/Cr/NAA/GLX series, Fig. 5.9 and Fig. 5.10, respectively. The simulated NAA/Cr/GABA series produced a concentration gradient of 0.96, 1.00, and 1.00 for the Govindaraju, Kaiser and Near models, respectively. For the NAA/Cr/GABA/GLX series, the gradient was found to be $0.93,0.98$, and 0.98 for the Govindaraju, Kaiser, and Near models, respectively. The addition of GLX to the simulation appears to make little difference to the quantification of the gradient, however the intercept of the concentration plots increases from - 0.05 for NAA/Cr/GABA, to -0.03 for NAA/Cr/GABA/GLX. This can likely be explained by the 2.1 PPM GLX peaks. These peaks will act to negate the negative NAA peak, reducing the constant scale factor on the GABA concentration gradient. The difference between the models appears small in this analysis, especially comparing Kaiser and Near, however, numerical integration is relatively insensitive to the effects of coupling. The model choice is anticipated to be more impactful for 

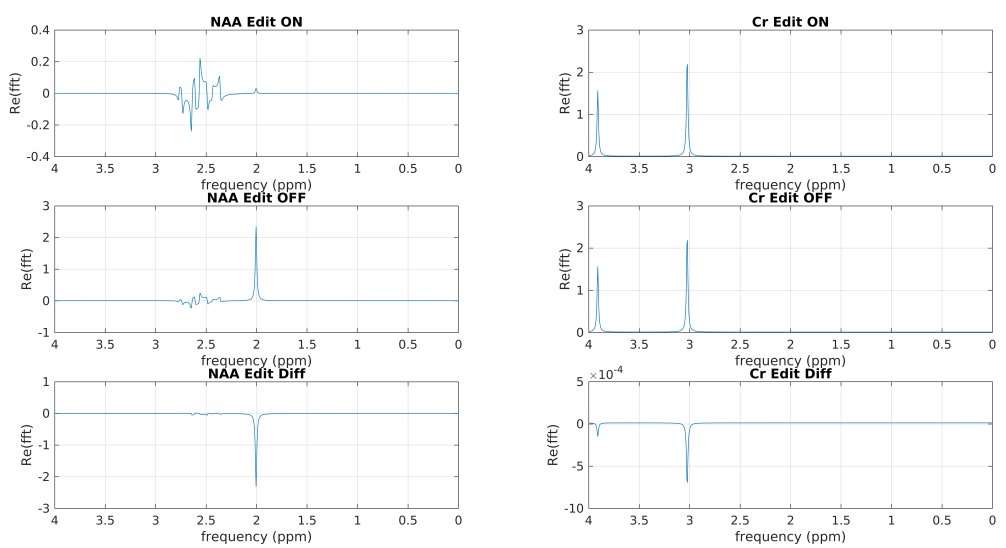

(a) NAA

(b) Creatine
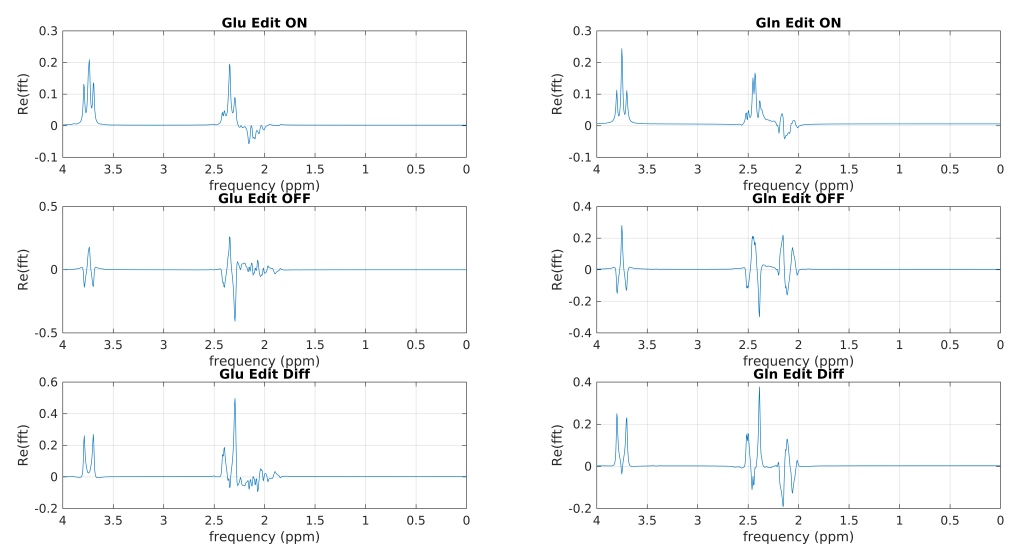

(c) Glutamate

(d) Glutamine

Figure 5.7: FID-A simulations of relevant metabolites for phantom series.

basis set fitting [143], where peak evolution is influenced by coupling structure. The choice of model will have further significance in the subsequent chapter, with molecular structure forming the basis of the methodology.

\subsection{Results}



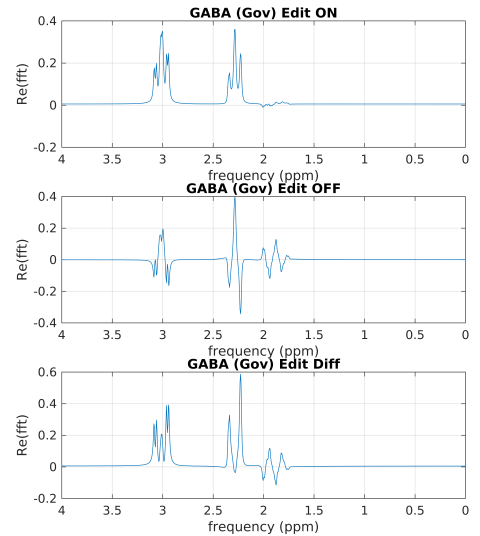

(a) GABA - Govindaraju
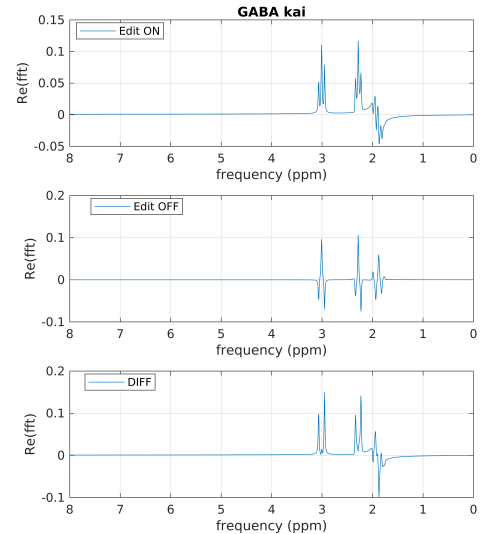

(b) GABA - Kaiser
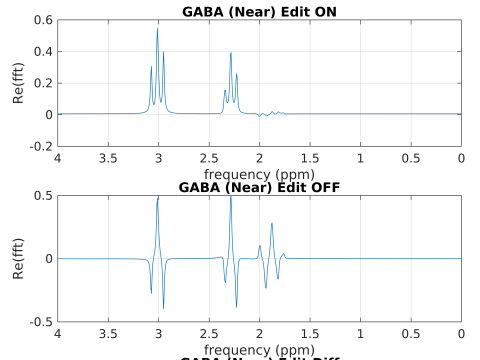

GABA (Near) Edit Diff

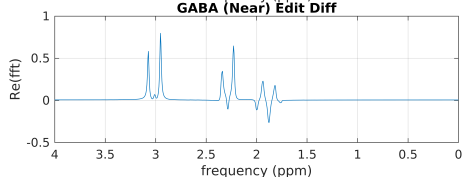

(c) GABA - Near

Figure 5.8: FID-A simulations for the three most commonly employed GABA models.

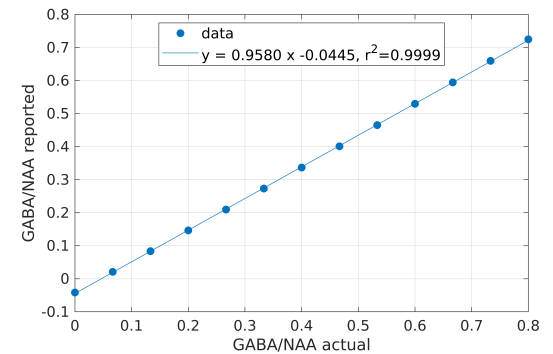

(a) Govindaraju et al.

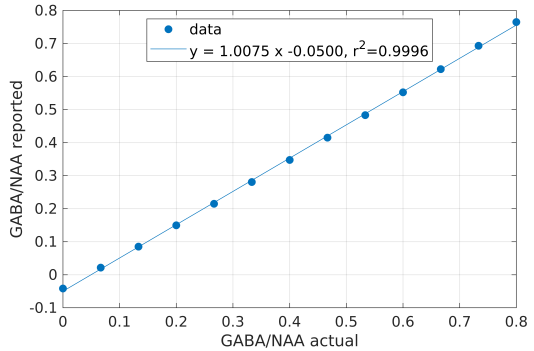

(b) Kaiser et al.

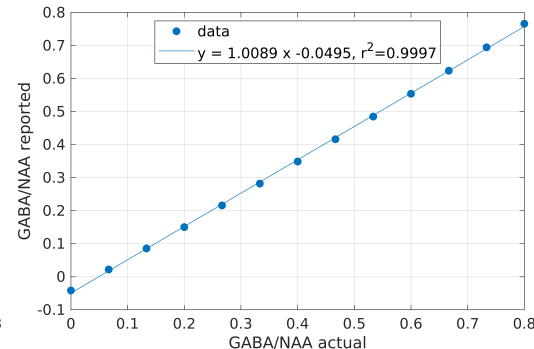

(c) Near et al.

Figure 5.9: Simulated GABA to NAA area ratio vs GABA concentration, for GABA/cr/NAA phantom, with linear fit for the three common GABA models.

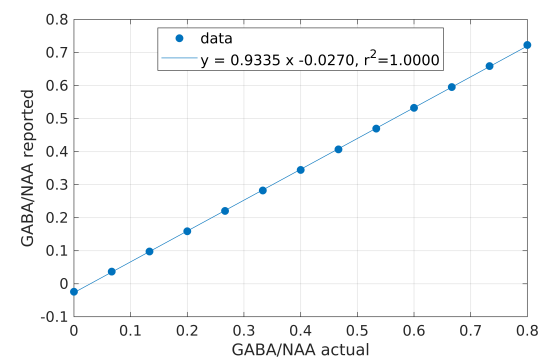

(a) Govindaraju et al.

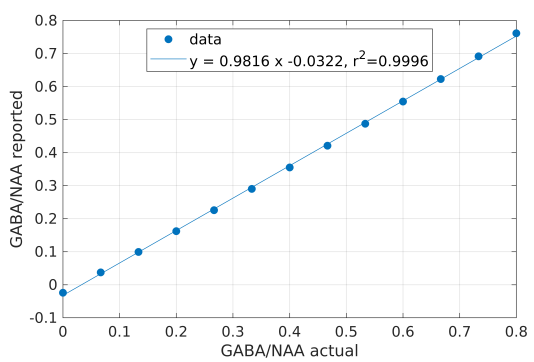

(b) Kaiser et al.

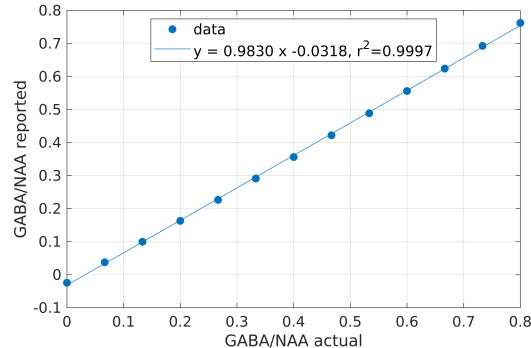

(c) Near et al.

Figure 5.10: Simulated GABA to NAA area ratio vs GABA concentration, for GABA/cr/NAA/GLX phantom with linear fit for the three common GABA models. 
For each analysis tool, the spectra are decomposed into a set of areas or amplitudes, as outlined in Sec. 5.3.3. The GABA amplitude alone will vary by acquisition, but taking the GABA signal as a ratio to the stable NAA peak will improve the quantification. This ratio is calculated for each tool, and plotted against the known concentration ratio of GABA to NAA. A linear fit is applied in each case, with the gradient and intercept extracted, along with the $R^{2}$ fit error. Plots of available data are included here, and full fit results are tabulated in Table. 5.4, and Table. 5.5.

The $R^{2}$ of the linear fits is found to vary by series, with well calibrated solutions exhibiting the highest, and the gel series the lowest.

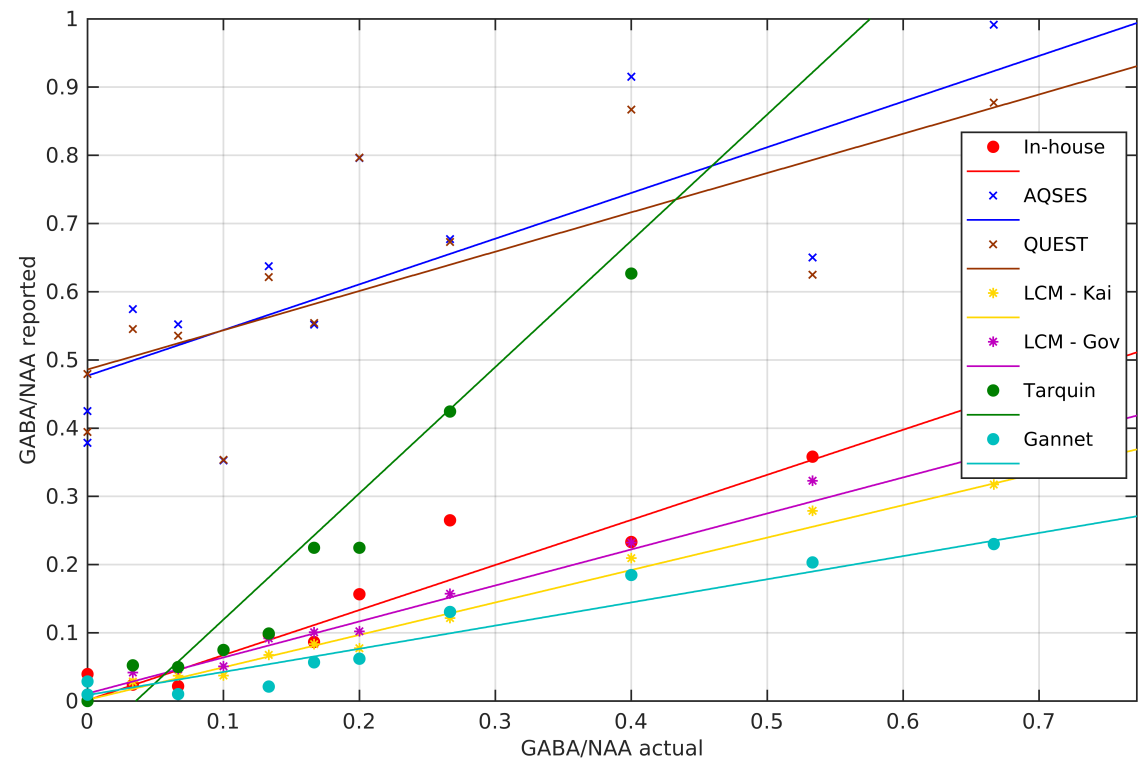

Figure 5.11: Measured GABA to NAA ratio vs known ground truth - GABA1.

Fig. 5.11 shows the linear fits for series GABA1. Perhaps the most notable feature of this plot is the Tarquin data, which appears to overestimate the GABA gradient by almost a factor of two. This is even more surprising when - with the exception of GANNET all other tools estimate the GABA gradient to be between 0.48 , and 0.66 . Tarquin does however display good linearity, with an $R^{2}$ of 0.98 . Another notable feature is the intercept of the JMRUI tools, QUEST and AQSES. While most tools are able to accurately ascertain the lower concentrations of GABA, the JMRUI tools report a GABA signal, where none is present. JMRUI also exhibits a lower $R^{2}$ than the other tools: 0.65 and 0.68 , versus a 
minimum of 0.93 for the others. While a lot of discrepancy between tools can be explained by varying approaches to preprocessing, the disparity between AQSES and QUEST can not. A common pre-processing was applied to each spectrum in JMRUI, so any differences between the two results are purely algorithmic in origin. For both solution series', LCModel's basis sets agree up to 1 decimal place, a surprisingly robust result. This suggests that preprocessing and algorithm have a larger impact than the basis set chosen. Fig. 5.12 shows

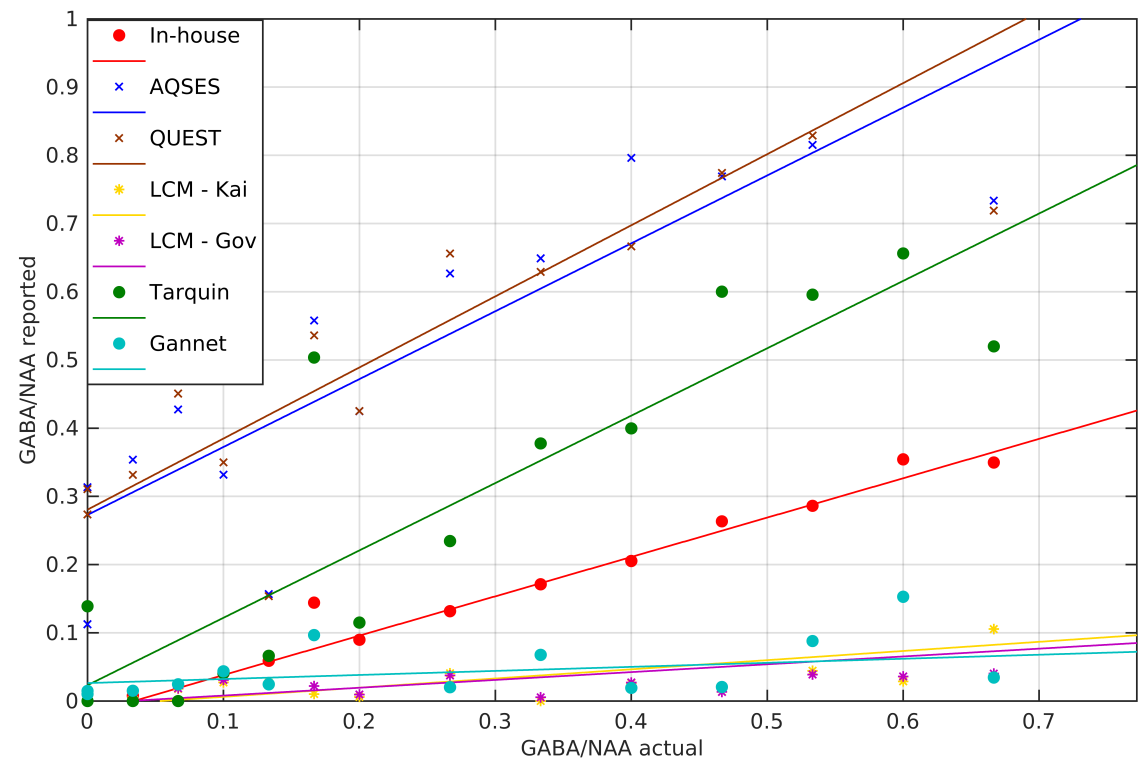

Figure 5.12: Measured GABA to NAA ratio vs known ground truth for the series GABA2.

the linear fits for the GABA2 series, where a low pH was maintained. Tarquin surprisingly performs better in this series, estimating a GABA gradient of 0.99 . While its $R^{2}$ is lower, the shift in the GABA gradient is appears to be a quirk of Tarquin's basis MEGA-PRESS basis set. LCModel and GANNET both severely underestimate the GABA gradient, with slopes of $0.11,0.13$, and 0.24 . The in-house Matlab based analysis maintains a high $R^{2}$ for both series', but underestimates the GABA gradient in both cases. For the in-house data, the analysis was repeated for 1, 2, 4, and 8 times zero-filled data, with the four-times data displayed in the figures, and full results in Table. 5.4, and Table. 5.5 Fig. 5.13, and Fig. 5.14 show the linear fits for body, and room temperature, respectively. For the gel series, GABA gradients and $R^{2}$ are generally lower, as a result of the more challenging environment. Despite this, Tarquin again over-estimates the GABA gradient, this time for room temperature series. 


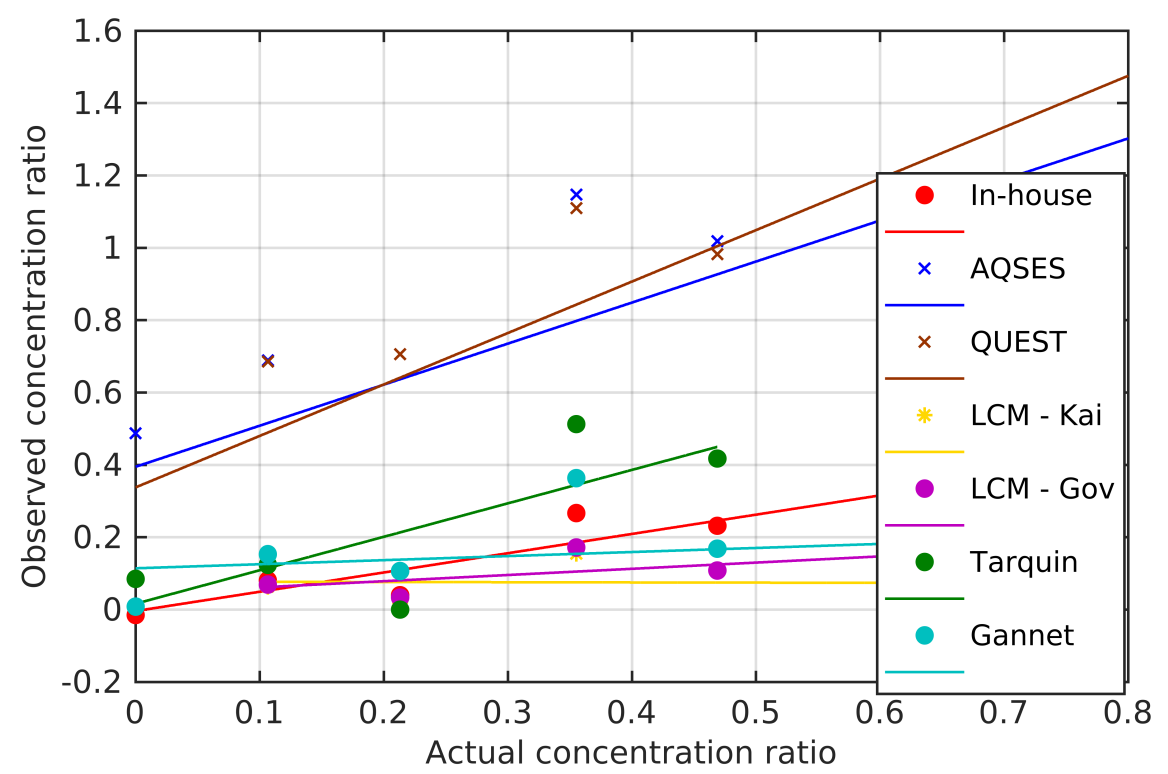

Figure 5.13: Measured GABA to NAA ratio vs known ground truth for the series GLX at body temperature.

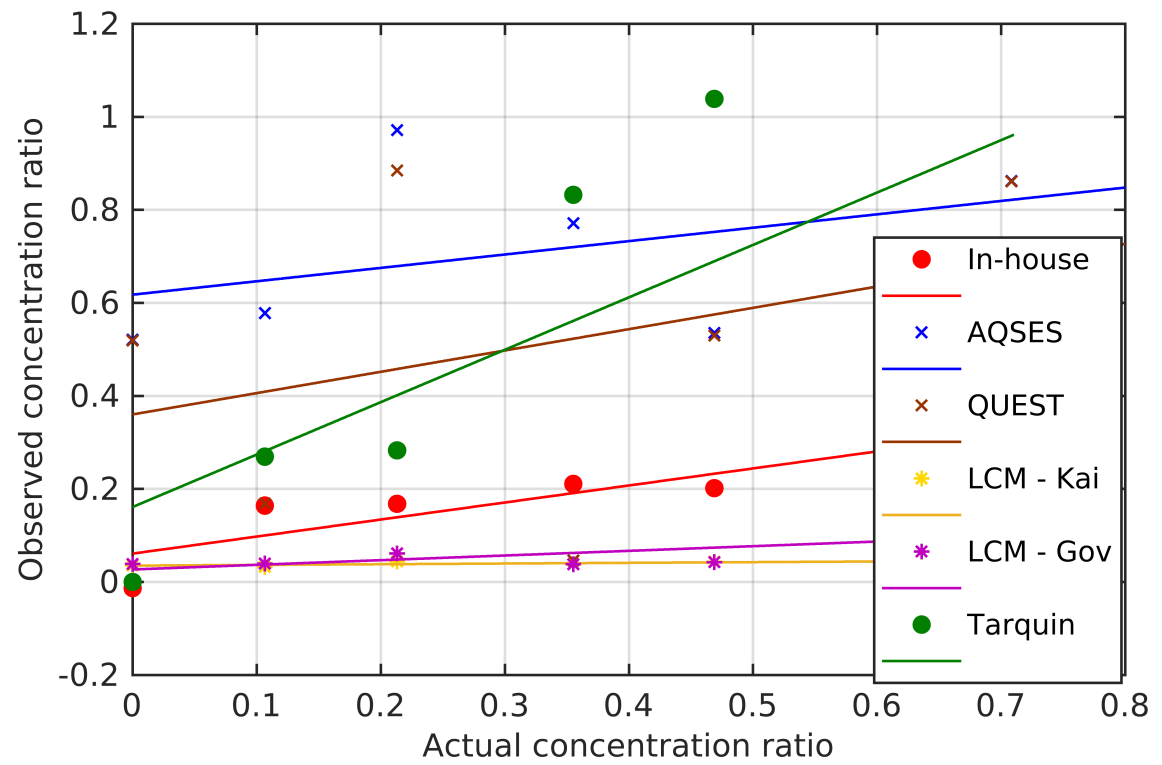

Figure 5.14: Measured GABA to NAA ratio vs known ground truth for the series GLX at room temperature.

LCModel performs poorly in the gel series', with $R^{2}$ lower than 0.55 in all cases. 


\begin{tabular}{|c|c|c|c|c|}
\hline Series & Basis & Gradient $(95 \% \mathrm{CI})$ & Intercept $(95 \%)$ & $R^{2}$ \\
\hline GABA1 & LCM - Gov & $0.5279(0.4873,0.5684)$ & $0.0111(-0.0034,0.0256)$ & 0.9868 \\
& LCM - Kai & $0.4756(0.4437,0.5075)$ & $0.0018(-0.0096,0.0132)$ & 0.9899 \\
& JMRUI AQSES & $0.6699(0.3662,0.9737)$ & $0.4769(0.3683,0.5854)$ & 0.6817 \\
& JMRUI QUEST & $0.5760(0.2976,0.8544)$ & $0.4860(0.3865,0.5854)$ & 0.6534 \\
& Tarqin & $1.8511(1.6879,2.0143)$ & $-0.0657(-0.1240,-0.0074)$ & 0.9826 \\
& Matlab x1 & $0.6440(0.5394,0.7487)$ & $0.0141(-0.0233,0.0515)$ & 0.9434 \\
& Matlab x2 & $0.6602(0.5610,0.7594)$ & $0.0012(-0.0343,0.0366)$ & 0.9512 \\
& Matlab x4 & $0.6607(0.5624,0.7591)$ & $0.0013(-0.0339,0.0364)$ & 0.9521 \\
& Matlab x8 & $0.6621(0.5646,0.7597)$ & $0.0008(-0.0340,0.0357)$ & 0.9530 \\
& Gannet3 & $-27.8761(-89.2030,33.4506) \times 10^{3}$ & $15.1674(-6.7416,37.0763) \times 10^{3}$ & 0.0834 \\
& Gannet3 excl 3 5 & $0.3396(0.2730,0.4062)$ & $0.0087(-0.0171,0.0345)$ & 0.9367 \\
\hline \multirow{6}{*}{ GABA2 } & LCM Gov red & $0.1146(0.0438,0.1854)$ & $-0.0035(-0.0308,0.0239)$ & 0.4626 \\
& LCM Kai red & $0.1344(0.0679,0.2009)$ & $-0.0073(-0.0330,0.0184)$ & 0.5731 \\
& JMRUI AQSES & $0.9952(0.7184,1.272)$ & $0.2729(0.1659,0.3798)$ & 0.8094 \\
& JMRUI QUEST & $1.042(0.712,1.372)$ & $0.2806(0.1529,0.4082)$ & 0.7660 \\
& Tarqin & $0.9878(0.7290,1.2465)$ & $0.0232(-0.0768,0.1232)$ & 0.8272 \\
& Matlab x1 & $0.5499(0.4593,0.6404)$ & $-0.0123(-0.0473,0.0227)$ & 0.9238 \\
& Matlab x2 & $0.5786(0.5229,0.6343)$ & $-0.0210(-0.0425,0.0005)$ & 0.9726 \\
& Matlab x4 & $0.5769(0.5211,0.6328)$ & $-0.0196(-0.0412,0.0020)$ & 0.9723 \\
& Gatlab x8 & $0.5761(0.5216,0.6306)$ & $-0.0183(-0.0393,0.0028)$ & 0.9735 \\
& Gannet3 & $0.2443(0.1949,0.2936)$ & $0.0082(-0.0108,0.0273)$ & 0.8894 \\
\hline
\end{tabular}

Table 5.4: Linear fit parameters for GABA1 and GABA2; the solution series. Gradients and intercept are reported with $95 \%$ confidence intervals, along with $R^{2}$ fit error.

\subsection{Discussion}

The results presented illustrate some of the shortcomings of some of the commonly used analysis methods. While both Tarquin and LCModel perform well for the PH calibrated solutions, when faced with the broader and noisier gel spectra, or the acidic solutions, they begin to produce more inconsistent results. LCModel in particular demonstrates this, for the GABA1 series, both basis sets have an $R^{2}$ of 0.99 . However, the gel series, LCModel is unable to determine the gradient, with $R^{2}$ less than 0.55 in all cases. This is particularly worrying, as the gel phantoms more accurately represent the in-vivo environment in which they are used. Tarquin does seem more robust with respect to these issues, but the large range of reported gradients is an issue, ranging from 0.55 for GLX, up to 0.98 for GABA1. 


\begin{tabular}{|c|c|c|c|c|}
\hline Series & Basis & Gradient (95\% CI) & Intercept (95\%) & $R^{2}$ \\
\hline GLX & LCM Gov red & $0.1714(-0.1589,0.5018)$ & $0.0442(-0.0965,0.1849)$ & 0.4763 \\
Body Temp & LCM Kai & $-0.0058(-0.4501,0.4385)$ & $0.0774(-0.1119,0.2667)$ & 0.0006 \\
& JMRUI AQSES & $1.135(-0.6976,2.967)$ & $0.3948(-0.3165,1.1060)$ & 0.4250 \\
& JMRUI QUEST & $1.422(0.2023,2.642)$ & $0.3378(-0.1358,0.8115)$ & 0.7237 \\
& Matlab x1 & $0.5218(0.2433,0.8002)$ & $-0.0024(-0.1106,0.1059)$ & 0.8712 \\
& Matlab x2 & $0.5274(0.2626,0.7923)$ & $-0.0044(-0.1074,0.0986)$ & 0.8843 \\
& Matlab x4 & $0.5328(0.2609,0.8047)$ & $-0.0040(-0.1097,0.1017)$ & 0.8810 \\
& Matlab x8 & $0.5348(0.2589,0.8107)$ & $-0.0038(-0.1111,0.1034)$ & 0.8787 \\
& Tarqin & $1.1061(-1.8532,4.0653)$ & $0.1850(-0.6548,1.0249)$ & 0.3205 \\
& Gannet3 & $0.1574(-0.0557,0.3705)$ & $0.0300(-0.0529,0.1129)$ & 0.5124 \\
\hline \multirow{2}{*}{ GLX } & LCM Gov red & $0.1002(-0.0246,0.2249)$ & $0.0267(-0.0218,0.0752)$ & 0.5541 \\
& LCM Kai & $0.0148(-0.0039,0.0336)$ & $0.0351(0.0278,0.0424)$ & 0.5474 \\
& JMRUI AQSES & $1.135(-0.3165,2.967)$ & $0.3948(-0.3165,1.106)$ & 0.4250 \\
& JMRUI QUEST & $1.422(0.2023,2.642)$ & $0.3378(-0.1358,0.8115)$ & 0.7237 \\
& Tarqin & $2.3385(-0.4145,5.0914)$ & $0.3122(-0.7583,1.3828)$ & 0.5817 \\
& Gatlab x1 & $0.3665(0.1063,0.6267)$ & $0.0592(-0.0420,0.1604)$ & 0.7927 \\
& Matlab x2 & $0.3682(0.1033,0.6332)$ & $0.0610(-0.0421,0.1640)$ & 0.7882 \\
& Matlab x4 & $0.3662(0.1051,0.6273)$ & $0.0609(-0.0406,0.1624)$ & 0.7913 \\
& Matlab x8 & $0.3630(0.1037,0.6222)$ & $0.0612(-0.0396,0.1620)$ & 0.7907 \\
& Gannet3 & $0.1123(-0.5081,0.7328)$ & $0.1142(-0.1271,0.3554)$ & 0.0594 \\
\hline
\end{tabular}

Table 5.5: Linear fit parameters for GLX gel series at room and body temperature. Gradients and intercept are reported with $95 \%$ confidence intervals, along with $R^{2}$ fit error.

The JMRUI results present a useful window into the algorithmic dependence of the quantification, independent of pre-processing. However, unlike the other tools presented, JMRUI has a large user dependence. Preprocessing, filtering, and frequency and phase calibrations are all handled by the user. Additionally, the basis set simulations are subject to user defined processing such as apodization, which can further affect fitting. While we acknowledge that an experienced user could enhance the performance of the tool, perhaps even beyond that of its competitors, the very fact that there is a user dependence is an issue in quantification, where results need to be reproducible across multiple scanners, patients, and acquisitions. The variability of results across all basis set tools illustrate the shortcomings of this method. Any deviation of the spectrum from its ideal line shape leads to misidentification of the concentrations. In-vivo, this effect is often masked by the sheer size of the basis sets. One of 
Tarquin's brain basis sets, for instance, contains thirty four individual models. Misidentification of one metabolite is often masked, as its signal is attributed to one of the other nearby model. The only non-basis set tool considered, GANNET, performs to a similar standard as the basis set methods. For GABA1, GANNET is found to estimate a large negative gradient, however, excluding two of the points brings the gradient from $-27.8761 \times 10^{3}$ with CI $(-89.2030,33.4506) \times 10^{3}$, to $0.3396(0.2730,0.4062)$. This value is in line with other tools, but the extremity of the outliers requires further investigation. When outliers are excluded, GANNET achieves a good $R^{2}$ for the solution series, however, it too underestimates the concentration. A surprising result is that simple in-house peak fitting method appears the most consistent across the series. $R^{2}$ is above 0.95 for all solution series, and 0.79 for the gel series. While still underestimating the true concentration ratios, the estimates are more consistent, and closer to the expected ratios than for any of the more sophisticated tools covered. This is a surprising result in itself. While perhaps not a viable option for standard spectroscopy sequences, simple fitting methods such as these can potentially be applied to specialist techniques such as MEGA-PRESS, and appear to provide a robust means of quantification. In this study, the manual analysis provides a good baseline for the experiment, and exhibits the presence of spectral information, even where more advanced methods are unable to detect it. This suggest the potential for over-processing of data, to the point where information is lost.

Closer inspection of the spectra suggest that spectra 2 and 3 in the room temperature GLX series suffer from poor $B_{0}$ homogeneity and poor water suppression, respectively. If these data points are excluded from the fit of the room temperature gel series, then the estimated concentration ratio increases to $30 \%$ of its actual value with $R^{2}$ of 0.95 . it also appears that the body temperature gels perform generally better, a surprising result, given the expected broadening of the peaks.

\subsection{Conclusion}

While basis set methods have proven more popular, the results of this study indicate a potential weakness of this analysis. Misidentification appears common, and in practice could be concealed by the presence of nearby models, leaving the user unaware of the issue. This 
effect is compounded for lower quality spectra, where the potential for misidentification increases. GANNET performs to a similar standard as the basis methods, across the solution series, but its performance drops considerably for the gel data. The relatively basic peak integration approach taken here, appears to be the most robust of the methods examined. This is surprising, and suggests over processing of the data, by other methods. While not feasible for standard in-vivo spectroscopy, these simple methods could have their place in analysis of specialist sequences such as MEGA-PRESS, and new techniques that can simplify the spectral landscape. This may be the analysis of choice for the techniques in the subsequent chapter.

This study could be easily extended to include more basis sets and quantification targets, and is considered a possible avenue of future work. Furthermore, a follow-up study using in-vivo data would allow more clinically relevant conclusions to be drawn. In general, our findings suggest a need for consistency. As with the relaxometry protocols, care must be taken when comparing more than one acquisition or analysis method, and standardisation is needed where possible. 


\section{Novel techniques for quantification of metabolites by MRS}

\subsection{Introduction}

Traditional RF pulses are tailored to produce a rotation, typically $90^{\circ}$, or $180^{\circ}$, to excite and refocus the magnetisation. MEGA-PRESS is one exception, using frequency selective pulses to edit the spectrum, however there are limitations to this technique. MEGA-PRESS uses the coupling of two resonances to generate signal in the difference spectrum, and is therefore limited in its potential application. It also requires twice as many acquisitions as a standard spectroscopy sequence, to produce a reduced peak amplitude in the difference spectrum.

Quantum optimal control is a general optimisation technique used to develop a set of control fields that can optimally manipulate a quantum system towards an established goal. The goal itself will vary by application, whether its achieving a state with minimised energy or time expenditure, or, more critically for us, moving a set of systems from an initial state, to some specified final state [144]. The general process involves quantum mechanical simulation of a system under the influence of a control field, then iterative refinement of that field until the system achieves its target state. The precise nature of the refinement procedure is defined by the quantum control algorithm, of which there are many variations. Control optimisation may be attempted in both the frequency domain [145] and time domain, for continuous or piece-wise constant controls. Controls formulated in the time domain, in a piecewise manner, are the focus here, where there are two main approaches; Krotov-type methods $[146,147]$ which update all controls within a single time slice before proceeding on to the next time slice, and GRAPE-type methods [148] which update all controls in all time slices concurrently [149]. Quantum optimal control is a relatively general technique and has been applied in many areas including the design of quantum gates $[150,151]$, trapped ions [152-154], and other spectroscopic applications [155-157].

Quantum control has also been applied to magnetic resonance, where the Bloch equations model the signal, and RF pulses are optimised as the control medium. Band selective pulses have been derived [158-160], as well as robust $90^{\circ}$ and $180^{\circ}$ pulses [161-163]. We propose a 
method of developing chemically selective RF pulses for magnetic resonance spectroscopy.

A magnetic resonance spectroscopy Hamiltonian, and full Liouville space, simulation package was implemented in Swansea, then incorporated into a quantum control framework by Max Chandler and Frank Langbein at Cardiff University. The structure of a given metabolite is modelled as a network of nuclear spins, characterised by their chemical shifts, and J-coupling to other spins within the network. The chemical shift and J-coupling may be quantified from spectra, usually derived from high resolution NMR. The models used in this study are cited, with sources, in Appendix. C. Simulations are restricted to systems with 7 spins or fewer, due to the exponential increase in computational cost as the system grows. There are methods capable of simulating large molecules, using approximations [164], but molecular structure is key to its controllability. The target state of a particular metabolite may be any (reachable) quantum state, but we want to define one that produces favourable spectroscopic features, i.e. the peaks of the target metabolite enhanced, while the peaks of interfering metabolites are simultaneously suppressed. We also need optimal pulses to account for a range of experimental instabilities. Variation in the local $B_{0}$ strength, will displace the chemical shifts, rendering unstable RF pulses ineffective. $B_{1}$ homogeneity will also vary, both spatially and temporally, deforming the effective pulse amplitude. Errors are estimated, and incorporated into target function, ensuring optimal pulses are robust with respect to these inhomogeneities. The theoretical basis for the simulation will be discussed first.

\subsection{Quantum mechanical simulation of MR pulse sequences}

\subsubsection{Hamiltonian models}

The simulations were formulated using a Hamiltonian model, where metabolites are represented as networks of nuclear spins, parametrised by their chemical shift and J-coupling. The Hamiltonian used was of the following form:

$$
\frac{1}{\hbar} H_{0}=\Sigma_{n} \epsilon_{n} \sigma_{z}^{(n)}+\Sigma_{m n} J_{m n}\left(\sigma_{x}^{(m)} \cdot \sigma_{x}^{(n)}+\sigma_{y}^{(m)} \cdot \sigma_{y}^{(n)}+\sigma_{z}^{(m)} \cdot \sigma_{z}^{(n)}\right),
$$

where $\epsilon$ is the chemical shift, $J_{m n}$ is the coupling between the $m$ and $n^{t h}$ spin, and $\sigma_{z}$ is the z Pauli matrix. The total Hamiltonian, $H$, is the sum of this system Hamiltonian, and the 
Hamiltonians defining external controls. The dynamics of the full system are described by the Schrödinger equation (6.2):

$$
i \hbar \frac{\partial}{\partial t}|\Psi\rangle=H|\Psi\rangle
$$

where $\hbar$ is Planck's reduced constant, $\Psi$ is the wavefunction. We may then write the wavefunction in terms of a unitary propagator, $U$ :

$$
\begin{gathered}
|\Psi(t)\rangle=U\left(t, t_{0}\right)\left|\Psi\left(t_{0}\right)\right\rangle, \\
U\left(t, t_{0}\right)=e^{\frac{-i}{\hbar} H\left(t-t_{0}\right)},
\end{gathered}
$$

where $t_{0}$ is the initial time, and $t$ is some later time. In MRS we do not deal with individual spin systems, we observe the cumulative effect of the contribution of many spin systems. In the following, we assume the $t_{0}=0$. This ensemble of spins is formulated in terms of a density matrix, $\rho$ :

$$
\rho=|\Psi(t)\rangle\left\langle\Psi(t)|=U(t)| \Psi_{0}\right\rangle\left\langle\Psi_{0}\right| U^{\dagger}(t),
$$

with the following equation of motion:

$$
\dot{\rho}(t)=-\frac{i}{\hbar}[H, \rho(t)] .
$$

Observations on this system are made by measuring the transverse magnetisation, perpendicular to the main field. In our framework this corresponds to the observables, $F_{x}$ and $F_{y}:$

$$
\begin{gathered}
F_{x}^{n}=I \otimes \sigma_{x} \otimes I, \\
F_{y}^{n}=I \otimes \sigma_{y} \otimes I,
\end{gathered}
$$

where the N-fold tensor product consists of N-1 identity matrices, with $\sigma$ as the nth factor. $\sigma_{x}$ and $\sigma_{y}$ are the Pauli $\mathrm{x}$, and y matrices respectively. In practice we measure the collective magnetisation of all spins, e.g. the sum over all $F_{x}^{n}$. Measurements performed upon these observables are achieved by taking the following traces:

$$
\begin{gathered}
\left\langle M_{x}\right\rangle=\operatorname{tr}\left(F_{x} \rho\right), \\
\left\langle M_{y}\right\rangle=\operatorname{tr}\left(F_{y} \rho\right),
\end{gathered}
$$

where $\rho$ is the density matrix describing the system prior to measurement. 


\subsubsection{RF fields}

Initially, the systems must be prepared in some state. This may be the ground state of the system, the lowest energy state, or some thermal state. A thermal state, $\rho_{0}$, for specific temperature, $T$, may be specified using Boltzmann statistics:

$$
\rho_{0}=e^{-\hbar H_{0} / K_{b} T}
$$

where $H_{0}$ is the system Hamiltonian, and $K_{b}$ is the Boltzmann constant. With an initial state specified, the next step is the simulation of the pulse sequence itself. The simulated sequences consist of pulses - either ideal rotations, or piece wise simulation of pulse shapes — and periods of free evolution, followed by observation. The simplest way to simulate an $\mathrm{RF}$ pulse is to reduce it to an ideal rotation in the $\mathrm{x}$ or $\mathrm{y}$ directions, respectively, represented by a unitary propagator:

$$
\begin{gathered}
U=e^{-i \theta F_{x}}, \\
U=e^{-i \theta F_{y}},
\end{gathered}
$$

where $\theta$ is the angle of rotation. This method instantaneously rotates the ensemble by the specified angle, however, it ignores the time-based evolution effects of the system. More faithful recreation of an $\mathrm{RF}$ pulse requires simulation of each time step from the piece-wise pulse. This achieved by generating a unitary operator for each time step in the pulse:

$$
U=e^{-2 \pi i H_{t o t} \Delta t}
$$

where $H_{t o t}$ is the total Hamiltonian; including the system Hamiltonian plus the control Hamiltonian. In between pulses, the system is allowed to evolve for some time, $\tau$, governed by the system Hamiltonian. This period of free evolution can be described by a single unitary operator:

$$
U=e^{-2 \pi i H_{0} \tau}
$$

After encoding all the pulses and evolution periods, we then need to make a measurement upon the system. In a real MRS experiment, measurement entails $N_{p t s}$ repeated signal acquisitions, over a specified dwell time, $T_{D}$. The acquired signal is therefore $N_{p t s} \times T_{D}$ in 
length. This is approximated by taking repeated observations of the operator, $F_{m}$ :

$$
F_{m}=F_{x}+i F_{y}
$$

i.e.

$$
\left\langle M_{m}\right\rangle=\operatorname{tr}\left(F_{m} \rho\right)
$$

and then evolving the system by the dwell time using Eq. (6.15). This process is then repeated $N_{p t s}$ times to construct the FID signal, which may then be Fourier transformed to acquire a spectrum for the system described by the Hamiltonian. FID, PRESS, STEAM, and LASER sequences were implemented in this fashion, as well as an array of common metabolic spin systems (Appendix. C).

\subsubsection{Dissipative simulation}

If the current Hilbert space simulation is allowed to propagate for some large time, the observed signal in $M_{m}$ will continue to oscillate, but never decay. In practice this is not what we see, a real system undergoes decoherence, and will relax back to its equilibrium magnetisation. This effect can be simulated by including dissipation. To properly simulate this dissipation, the dynamics must be reformulated in Liouville space. We define the Liouvillian:

$$
\mathcal{L}=-i\left[H_{0}\right]+\Sigma_{n}\left(R_{1} \mathcal{D}\left(\sigma_{-}^{(n)}\right)+R_{2} \mathcal{D}\left(\sigma_{z}^{(n)}\right)\right)
$$

where $R_{1}$ and $R_{2}$ are the inverse of $T_{1}$, and $T_{2}$ respectively. $\sigma_{-}$is the lowering operator.We define the super-operator, $\mathcal{D}$, acting on some operator, $v$ :

$$
\mathcal{D}(v)=v \rho v^{\dagger}-\frac{1}{2}\left(v^{\dagger} v \rho+\rho v^{\dagger} v\right) .
$$

resulting spectra are broader, and lower in amplitude do de-phasing, more in line with experimental data than the sharp resolved peaks. This method also allows the relaxation dynamics with a pulse to be captured. However, this extra accuracy comes at a cost. For Hamiltonian simulations, the size of the density matrix for an $N$ spin system scales with $2^{N}$.

For a Liouville system, the matrix size scales with $\left(2^{N}\right)^{2}$. For larger spin systems, such as NAA, a single sequence simulation can be on the order of several hours. For this reason, it 
was deemed infeasible to use dissipative simulations during the optimisation process. Dissipation is included during the robustness analysis of pulses, so as to reduce the number of calculations, while still examining the effects.

\subsection{Optimisation using optimal control}

\subsubsection{Theory of optimal control}

The evolution of the controlled system is governed by a total Hamiltonian, $H$, which is dependent upon the system Hamiltonian, $H_{0}$ (Eq. (6.1)), and some other RF dependent component. The RF pulse is modelled as a time-varying control field, $\mathbf{f}(t)$, where $\mathbf{f}$ is a classical field. $H$ may be represented as a linear perturbation of $H_{0}$ by a set of control Hamiltonians, $H_{m}$. The control Hamiltonians are formulated as an expansion of the $\sigma_{x}$ and $\sigma_{y}$ Pauli matrices, representing coils in the $x$ and $y$ dimension, respectively. The total Hamiltonian is the sum of these controls and the system Hamiltonian:

$$
H(t)=H_{0}+\sum_{m=1}^{M} f_{m}(t) H_{m} .
$$

For algorithmic purposes, time will be discretised, either implicitly, or explicitly. The $M$ time-independent control Hamiltonians, have a corresponding time-dependent control vector, $f_{m}$, where $f_{m}$ has a discrete amplitude for each time step. Here we have formulated the problem in terms of discrete time steps. There are many ways to approach this problem, we examined two such approaches. State transfer problems seek to find a field, $\mathbf{f}$, that transfers the system from its initial state, $\rho(0)$, to some specifically defined target state, $\rho_{\text {tar }}$, by some time, $T$. The success of the control is defined by a transfer fidelity, $\mathcal{F}$ :

$$
\mathcal{F}(\mathbf{f})=\operatorname{tr}\left(\rho_{\text {tar }} \rho(T)\right)
$$

Transfer fidelity is maximised when the Hilbert-Schmidt distance is minimised. An error functional, $E$, may be defined, accordingly:

$$
\begin{aligned}
E(\mathbf{f}) & =\frac{1}{2}\left\|\rho(T)-\rho_{t a r}\right\|_{S}^{2}, \\
& =E_{0}-\mathcal{F}(\mathbf{f}),
\end{aligned}
$$


where $E_{0}$ is a constant with value $\operatorname{tr}\left(\rho_{0}^{2}\right)+\operatorname{tr}\left(\rho_{\text {tar }}^{2}\right)$. This is perhaps less intuitive, as the target is defined as a specific quantum state, rather than a physical observable. However it is also possible to optimise in this way. Observable optimisation problems search for controls that deliver a certain expectation value from an observable operator, $Q$. The transfer fidelity in this case is given by:

$$
\mathcal{F}(\mathbf{f})=\operatorname{tr}(Q \rho(T))
$$

A similar form to Eq. (6.21), but distinct in the formulation of the target. Full details may be found in calculations by Shermer et al. [165]. In the simulation of pulse sequences, the quantum mechanical propagator can become very time consuming to compute. In situations such as these, local gradients are often calculated to reduce the number of computationally expensive function calls [166]. In our case optimisation is performed using a box-constrained, limited-memory variant of the BFGS algorithm, L-BFGS-B [167]. The controls are then updated concurrently to reduce the impact of propagator calculations on computational load. The gradients themselves may be calculated analytically. The exact analytic gradients for state transfer problems has been studied extensively in other arenas, and so the solutions are readily available. Machnes et al. [149] found the derivative for the gradient to be:

$$
\frac{\partial f\left(U\left(t_{k}\right)\right)}{\partial u_{j}}=\frac{1}{c} \operatorname{Re}\left(\operatorname{tr}\left\{\rho_{\text {tar }}^{\dagger}\left(t_{k}\right)\left(\frac{\partial U_{k}}{\partial u_{j}}\right) \rho_{0}\left(t_{k}-1\right) U_{k}^{\dagger}\right\}+\operatorname{tr}\left\{\rho_{t a r}^{\dagger}\left(t_{k}\right) U_{k} \rho_{0}\left(t_{k}-1\right)\left(\frac{\partial U_{k}^{\dagger}}{\partial u_{j}}\right)\right\}\right),
$$

with $\frac{\partial U_{k}}{\partial u_{j}}$ as follows :

$$
\left\langle\lambda_{l} \mid \frac{\partial U}{\partial u_{j}} \lambda_{m}\right\rangle= \begin{cases}-i \Delta t\left\langle\lambda_{l}\left|H_{j}\right| \lambda_{m}\right\rangle e^{-i \Delta t \lambda_{l}} & \text { if } \lambda_{l}=\lambda_{m} \\ -i \Delta t\left\langle\lambda_{l}\left|H_{j}\right| \lambda_{m}\right\rangle \frac{e^{-i \Delta t \lambda_{l}-e^{-i \Delta t \lambda_{m}}}}{-i \Delta t\left(\lambda_{l}-\lambda_{m}\right)} & \text { if } \lambda_{l} \neq \lambda_{m}\end{cases}
$$

$\lambda$ are the Eigenvectors of the Hamiltonian, $U$ are the unitary propagators, and $j$ and $k$ are indices. The specifications of the optimal pulses are also limited to the capabilities of our scanner. RF amplitudes, pulse duration and resolution are all constrained within specifications of our 3 Tesla Siemens Magnetrom, where the maximum pulse amplitude is capped at $800 \mathrm{rads}^{-1}$, and minimum scanner transmit time is set to $25 \mathrm{~ns}$. 


\subsubsection{Target state selection, objective functions, and constraints}

The optimal choice of target state for a chemically selective RF pulse is not immediately obvious. Initial attempts at identifying GABA selective pulses involved simply optimising for maximal observed GABA signal, while suppressing that of the other peaks. As per Eq. (6.24), the spectrum was acquired, and the integral of the total signal profile used as a figure of merit. While in principle this seems sensible, algorithmically it is an ill defined target, with the algorithm unable to converge to a high fidelity. As such, in the second instance, optimisation was reformulated as a state transfer problem. There are many potential choices of target state that can provide favourable results. We attempted to optimise an excitation of a subset of spins within a molecule. It is possible to construct states where individual constituent spins of a system lie in different quantum states. For example, for a three spin system we can generate a state where only the first spin is excited:

$$
|\Psi\rangle=\frac{1}{\sqrt{2}}(|\uparrow\rangle+|\downarrow\rangle) \otimes|\downarrow\rangle \otimes|\downarrow\rangle
$$

Fig. 6.1 shows several simulated GABA spectra (using the model by Govidaraju et al. ), whereby the spin system was initialised with one of its six spins in an excited state, while the other five remain in the ground state. The spectrum in each case is a modulated singlet, with the other resonances producing little to no signal over the readout duration. Initial optimisation targets were selected by manually selecting spins within a molecule to excite and suppress. In one case we attempted to generate a control to simultaneously excite a sub group of spins in the creatine and GABA models. For the GABA spin system, only the resonances at 2.28 PPM were to be excited, and only the 3.91 PPM resonance of creatine. While perhaps not an ideal in-vivo pulse, it would eliminate any overlap in the signal of the two metabolites. The current target states are automatically identified, chosen as a combination of excited protons that maximise the separation of resulting resonances. If multiple protons of a given system are within $\pm 0.05 \mathrm{PPM}$ of the chosen peak, they are also are added as an excitation target. 


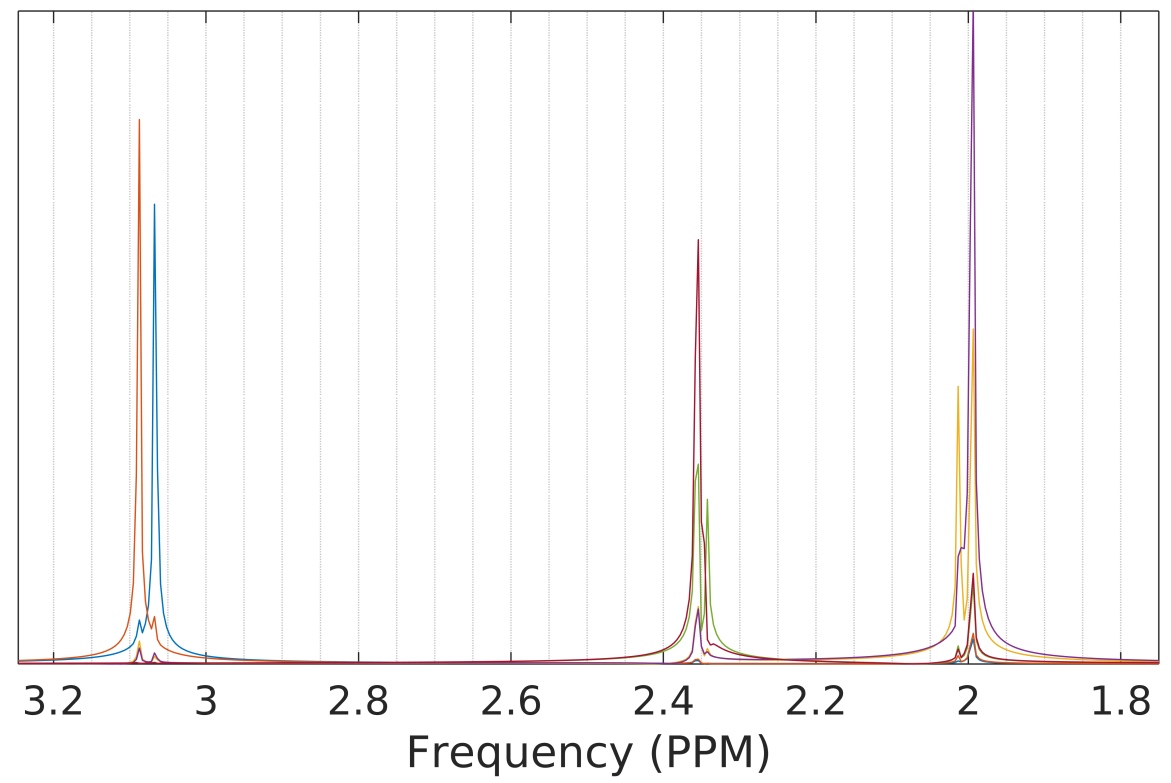

Figure 6.1: Simulated GABA spectrum. Single spins are excited and an FID immediately acquired.

\subsubsection{Robustness considerations}

Within the discussion so far, pulses may be identified that allow theoretical discrimination of previously indistinguishable spectra. While the simulated control may achieve a high fidelity state transfer, experimental implementation brings with it many additional influences that are not present in the basic Hamiltonian simulation. The most critical factors are the inhomogeneities of the $B_{0}$ and $B_{1}$ fields. The Hamiltonian simulation assumes no offresonance effects, and any deviation from this may destroy the selectivity of some pulses.

Inhomogeneity in the $B_{0}$ field will shift spins from a single resonance frequency, to a Gaussian distribution of Larmor frequencies. In the spectra this is tantamount to the chemical shifts becoming distributed about some mean, and manifests itself as peak broadening. This is integrated into the optimisation process by repeated simulation of the system with an additional Hamiltonian component, adding a $\sigma_{z}$ resonance offset. The range and resolution of $B_{0}$ to be investigated will inevitably increase the computational cost of simulation, so must be balanced with efficiency. Each set of metabolites is simulated up to $\pm 10 \mathrm{~Hz}$ off resonance, at $1 \mathrm{~Hz}$ intervals. $\mathrm{RF}$ pulses are tested across this full range, and are required to 
maintain state transfer fidelity at each point.

$B_{1}$ inhomogeneity is a more complex issue. Where $B_{0}$ instabilities affect the distribution of chemical shifts, $B_{1}$ effects manipulate the pulse itself in both spatial distribution, modulating the effective flip angle, and temporal profile, distorting the features of the pulse. $B_{1}$ variation is implemented with three facets, effective flip angle, noise, and RF amplification error. While the term flip angle is perhaps less applicable to a chemically selective pulse, the underlying principle of pulse energy is. The optimised pulse is assumed to possess an effective $90^{\circ}$ flip angle, and is simulated with $\pm 5^{\circ}$, in $1^{\circ}$ intervals, scaling the pulse energy. Noise is incorporated as a normally distributed, random perturbation of each time step. The degree of perturbation is scaled as a percentage of the amplitude, varied from $1 \%$ to $10 \%$, in $1 \%$ increments. The resulting spectra are averaged over ten repeat runs before analysis. Optimised RF pulses can have complex, rapid varying pulse profiles. While the scanner limitations are accounted for in optimisation, we may still observe distortion of the pulse during amplification. RF amplifier error is approximated by a Savitzky-Golay, polynomial filter applied to the pulse. The filter smooths the RF pulse profile, affecting high frequency variations more. The pulse is filtered with varying bucket size $(1,5,7,9,11,13)$, and the fidelity examined in each case.

Finally, the performance of the pulse is assessed under full dissipative simulation. While it is too computationally expensive to model dissipation during the optimisation process, it will still have an effect, in particular $T_{2}$ dissipation. $T_{1}$ and $T_{2}$ times of the models are estimated from literature values [168-170], then varied between $80 \%$ and $120 \%$ of this value, in $5 \%$ increments. in each case, the state transfer fidelity is assessed.

\subsubsection{Localisation}

The current optimisations are performed for a simple FID sequence, where the system is prepared in the ground state, the control pulse applied, followed immediately by readout. While this is deemed sufficient for initial experimental implementations, the neglect of any localisation scheme renders such pulses less effective in-vivo. Furthermore, including the current optimised pulses into a standard localisation scheme like PRESS or STEAM will destroy selectivity during refocussing and long evolution times. It may be possible to include 
some form of conventional localisation scheme into the optimisation process, whereby the simulation is expanded to an entire sequence, and refocussing pulses designed to maintain selectivity. However, this will come at a significant computational cost, and will further reduce the search space of the algorithm, perhaps making it unrealistic, at least in the first instance.

One potential solution we propose is outer volume suppression, to be used in conjunction with the FID sequence we have optimised for. Outer volume suppression utilises slice selective RF pulses to excite all spins outside the selected voxel. This is closely followed by a set of strong crusher gradients to de-phase the signal, in a similar vein to CHESS water suppression. Outer volume suppression is most commonly used to augment other localisation schemes [171], to reduce the effects of spurious signals originating outside the target voxel. However, more recent work has vindicated the use of outer volume suppression as a localisation scheme in its own right [172-174].

\subsection{Optimisation results}

\subsubsection{Theoretical evaluation of optimised pulses}

The optimisation framework has generated hundreds of pulses for various targets, and with varying success. The full analysis of these results is beyond the scope of my own thesis, so only selected examples are discussed here. Fig. 6.2, and Fig. 6.3 show the pulse profiles of two successful optimisation results. "GABA 106", and "GABA 147" were optimised to discriminate between GABA and creatine. GABA 106 aims to suppress the 3.0 PPM peak creatine, and concurrently maximise the signal of the 2.3 PPM resonance of GABA, while suppressing the others. The GABA 147 pulse also suppresses creatine, but this time exciting the 1.8 PPM resonance of GABA. While these choices may well not be the optimal for invivo data acquisitions, they are adequate for illustrative purposes, and proof of principle. Both pulses appear surprisingly simple in their real and imaginary profiles, exhibiting high frequency modulations embedded into lower frequency line shapes. However more complex features are observed in the phase of both pulses, with several large switches from positive to negative phase. To visualise the effects of these pulses, their action upon GABA and creatine 


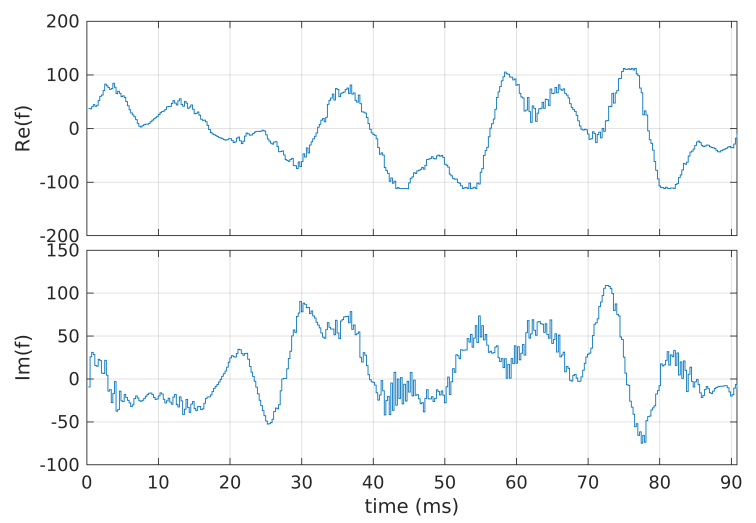

(a) Real and imaginary

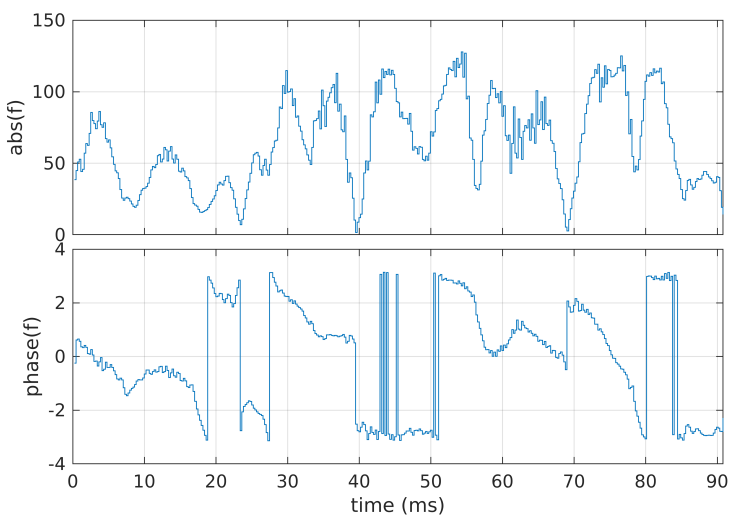

(b) Magnitude and phase

Figure 6.2: Optimised pulse: "GABA 106".

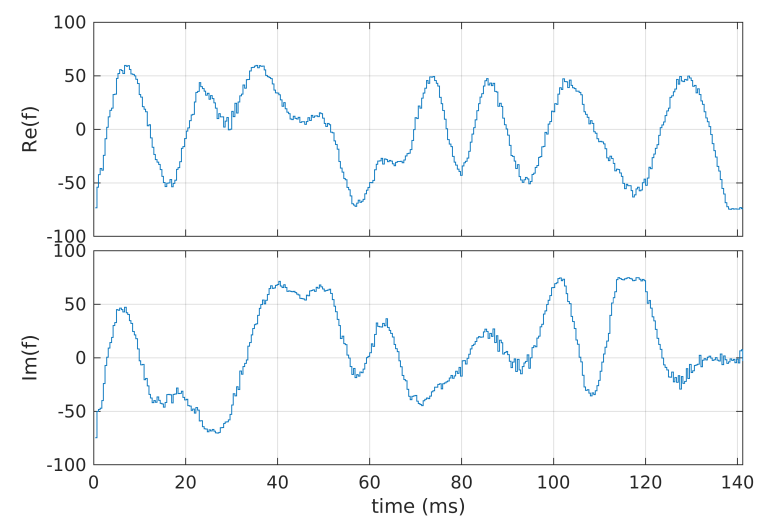

(a) Real and imaginary

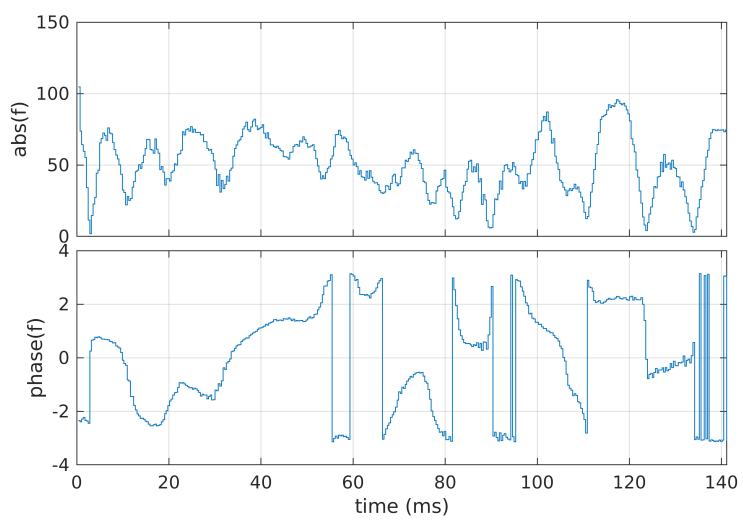

(b) Maginitude and phase

Figure 6.3: Optimised pulse: "GABA 147".

is simulated. Fig. 6.4 shows the simulation results for creatine under influence of each pulses, as well as the ideal FID, for comparison. GABA 106 produces a $99 \%$ reduction in the 3 PPM creatine resonance, and a 90\% reduction in the 3.9 PPM resonance, compared to that of the FID. Similarly, the GABA 147 pulses produces a 96\% reduction of creatine at 3 PPM, and 95\% at 3.9 PPM. Both pulses are able to successfully suppress the creatine signal at 3 PPM to $5 \%$ it's original value. These results were generated using the Govindaraju model for GABA. To investigate the effects of model choice, the optimal pulses were simulated for all three GABA models considered here; Govindaraju, Kaiser, and Near. Fig. 6.5 shows the results for the Govindaraju model. Optimised pulse GABA 106 reduces the magnitude of 


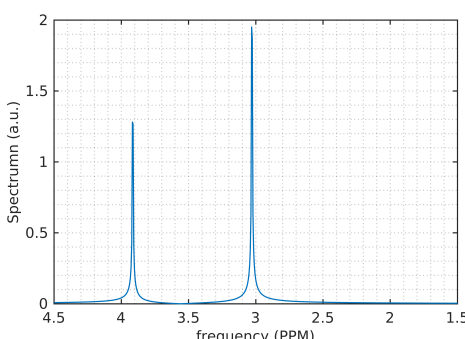

(a) Ideal

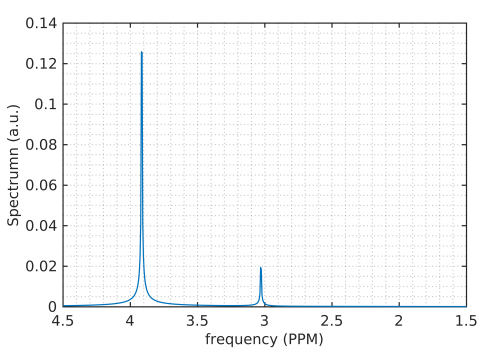

(b) GABA 106

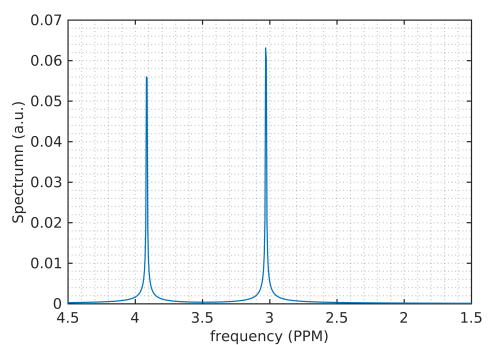

(c) GABA 147

Figure 6.4: Spectra for ideal sequence, and two optimised pulses for creatine.

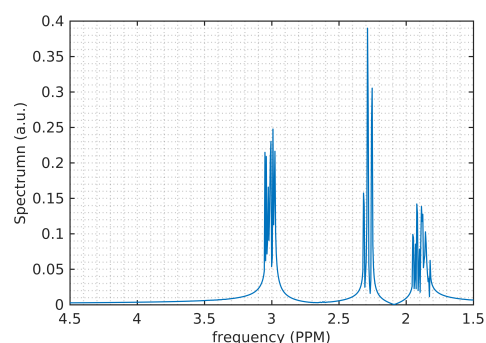

(a) Ideal

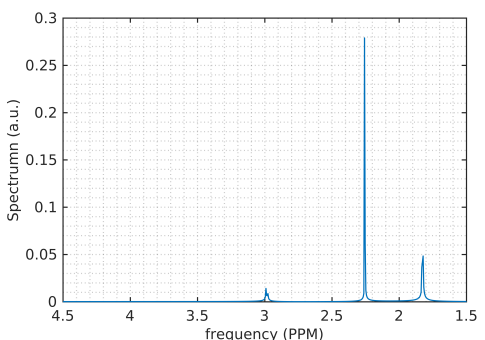

(b) GABA 106

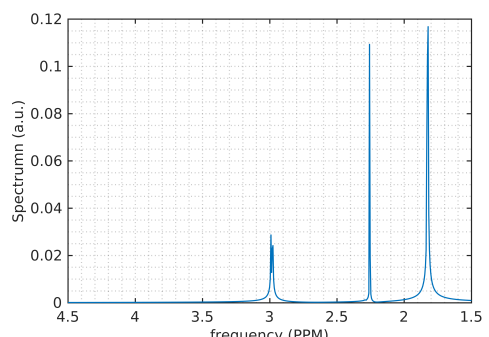

(c) GABA 147

Figure 6.5: Spectra for ideal sequence, and two optimised pulses for Govindaraju GABA model.

the 3 PPM GABA resonance by $94 \%$, a similar fidelity to that of the creatine. The target 2.3 PPM resonance of GABA is also reduced by the pulse, but only by around $28 \%$. The resulting spectrum is effectively a single resonance at 2.3 PPM, and this single component is reflected in the relatively simple FID. Imperfect excitation, and J-coupling effects during readout, mean some degree of signal loss is almost inevitable. For pulse GABA 147, the 2.3 PPM peak is reduced by $72 \%$, the 3PPM peak by $91 \%$, and the 1.8 PPM resonance by only $16 \%$. In the case of the Kaiser model, GABA 106 reduces the 2.3 PPM peak by $62 \%$, while the 3 PPM peak is suppressed by $98 \%$. For GABA 147, the 2.3 and 3 PPM peaks are reduced by $86 \%$, and $96 \%$, respectively. GABA 147 reduces the 1.8 PPM resonance by $78 \%$ for the Kaiser model. Finally, for the Near model, GABA 106 reduces the 2.3 and 3 PPM peaks by $61 \%$ and $97 \%$, respectively. While the GABA 147 reduces them by $85 \%$, and $93 \%$, respectively. The $1.8 \mathrm{PPM}$ resonance is reduced by $65 \%$. As expected, the choice of 


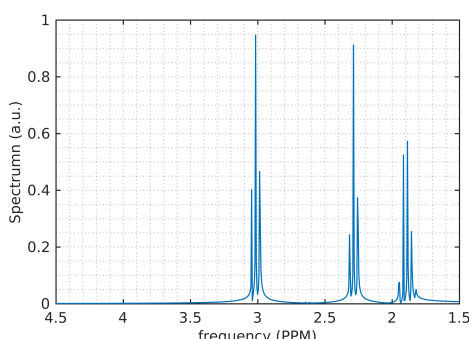

(a) Ideal

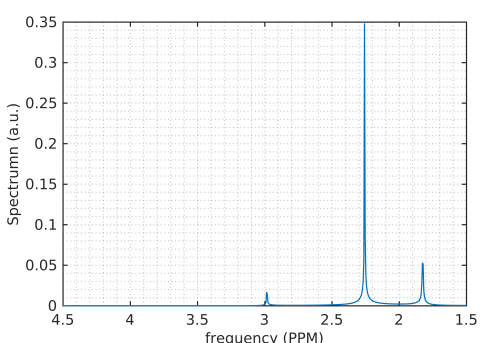

(b) GABA 106

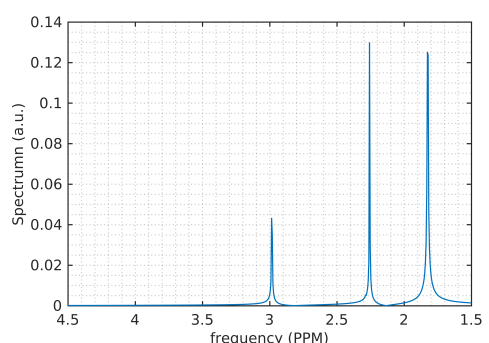

(c) GABA 147

Figure 6.6: Spectra for ideal sequence, and two optimised pulses for Kaiser GABA model.

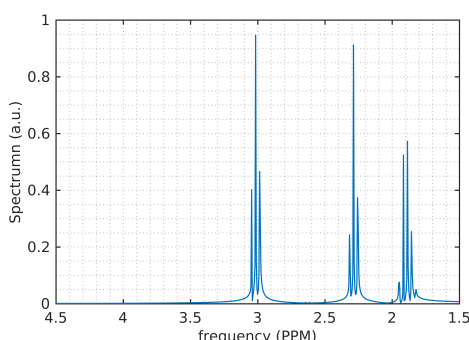

(a) Ideal

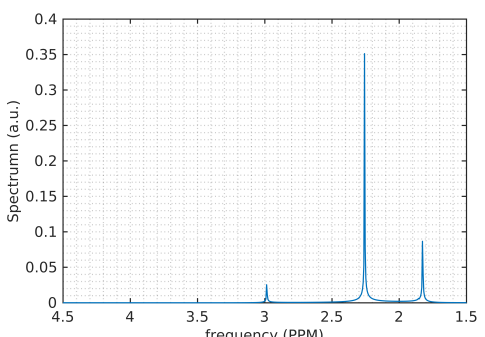

(b) GABA 106

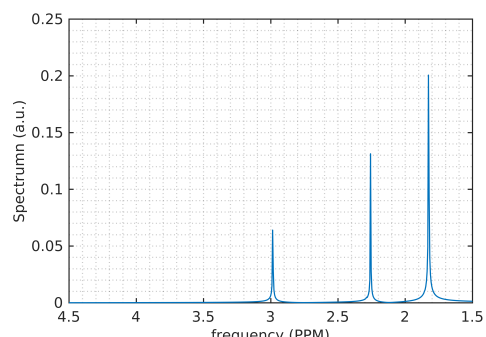

(c) GABA 147

Figure 6.7: Spectra for ideal sequence, and two optimised pulses for Near GABA model.

GABA model does indeed affect the fidelity of the RF pulse. While selectivity may still be seen to varying extent, the pulses make use of the minor differences in coupling structure to drive selectivity. This selectivity is inevitably lost when said structure changes. This again highlights the need for accurate metabolite models.

While a frequency selective pulse from a MEGA sequence will only affect resonances within its edit bandwidth, these optimised pulses will affect all spins, and the resulting spectrum is non-trivial. The results presented here were aimed at discriminating $\mathrm{Cr}$ and GABA, but in order to quantify these results, other overlapping resonances must be considered. Fig. 6.8 shows the affect of these pulses on glutamate, glutamine, and NAA. Generally the optimised pulses appear to reduce the magnitude of all resonances to varying degrees. The complex structure of the optimised pulse does generate excitations outside of the intended target, but the excitation is not optimal, so the resonances are drastically reduced in magnitude. In principle this is positive, however the same process will occur in the intended 


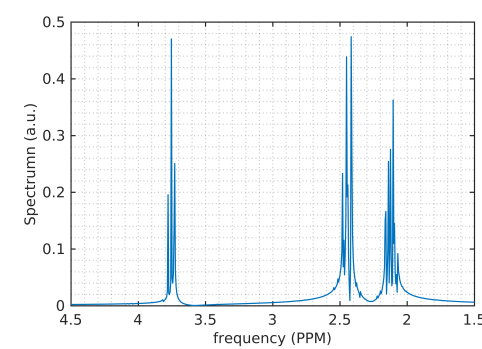

(a) Glu - Ideal

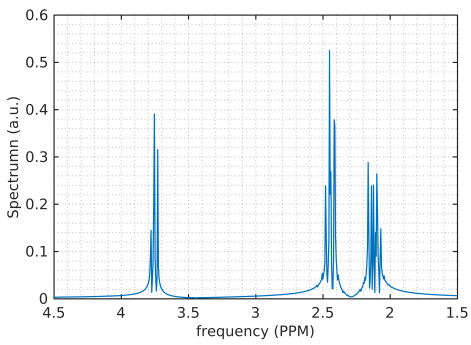

(d) Gln - Ideal

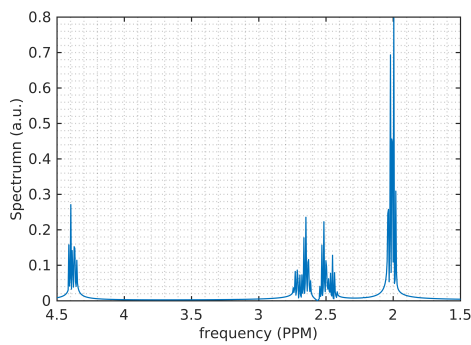

(g) NAA - Ideal

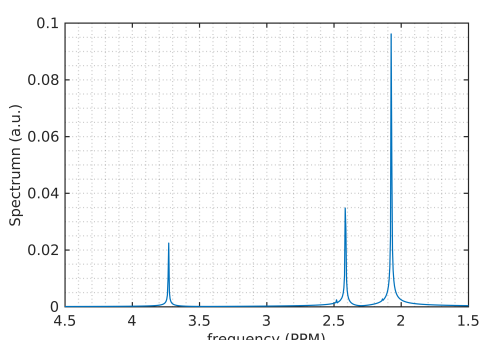

(b) Glu - GABA 106

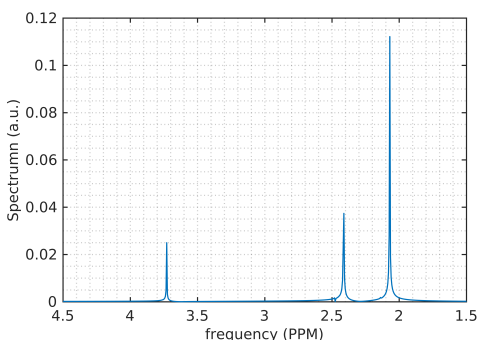

(e) Gln - GABA 106

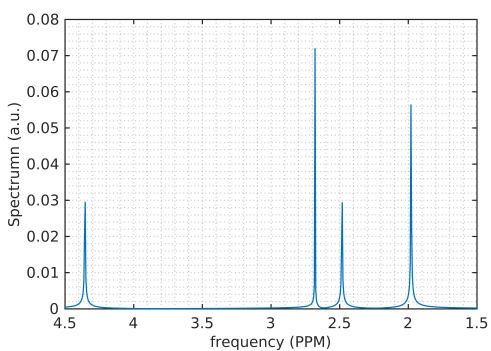

(h) NAA 106

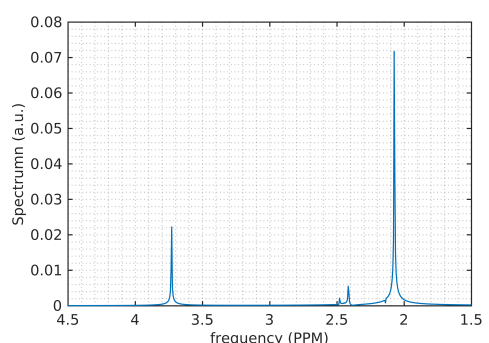

(c) Glu - GABA 147

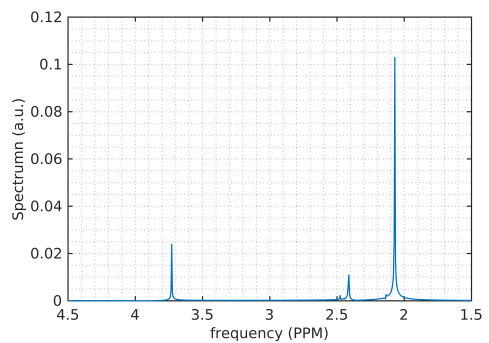

(f) Gln - GABA 147

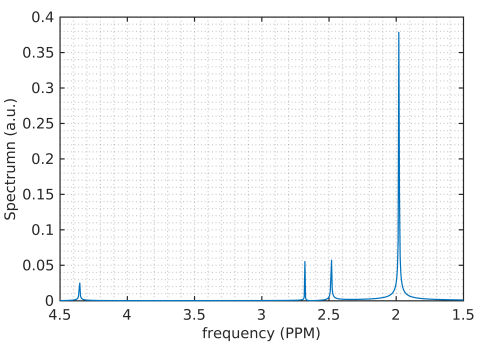

(i) NAA 147

Figure 6.8: Spectra of optimised pulses for glutamate, glutamine, and NAA.

targets if the models used in optimisation are not accurate.

\subsubsection{Experimental evaluation of optimised pulses}

Optimised pulses were implemented into basic FID spectroscopy sequence using the Siemens IDEA pulse programming environment by Sophie Shermer. The optimised RF pulses were uploaded to the scanner's pulse library, and the standard FID sequence modified to utilise them. The water suppression scheme was unchanged, and localisation can, in principle, be achieved using outer volume suppression. Optimised pulses have amplitudes specified in units of Rabi frequency or $\operatorname{rad~s}^{-1}$. These units must be converted to transmit voltages, requiring 
calibration of the transmitter to determine the correct settings. This is complicated by the fact that these voltage settings depend on the dielectric and RF absorption properties of the phantom, its placement relative to the transmitter, and the characteristics of the hardware itself. Transmitter calibration is a common technique, however, conventional transmitter calibration — based on stimulated to spin echo ratios - is not possible here. A simplistic calibration we can perform is to fix the pulse shape, duration, and all other parameters, and systematically vary transmitter voltage.

For a non-selective excitation pulse, we expect some variation in peak amplitudes, especially for very low voltages, but the relative heights of a peak should remain stable for a given metabolite. This is exactly what we observe in Fig. 6.9(a), for a standard FID sequence, with a $400 \mu$ s broadband RF excitation pulse. The broadband pulse excites all transitions and the three characteristic clusters of GABA peaks are visible in all spectra, except at extremely low voltages, where the peaks are obscured due to very low SNR. The stability of the spectrum with respect to transmitter voltage is desirable, it demonstrates the robustness of the pulse with respect to $B_{1}$ inhomogeneity.

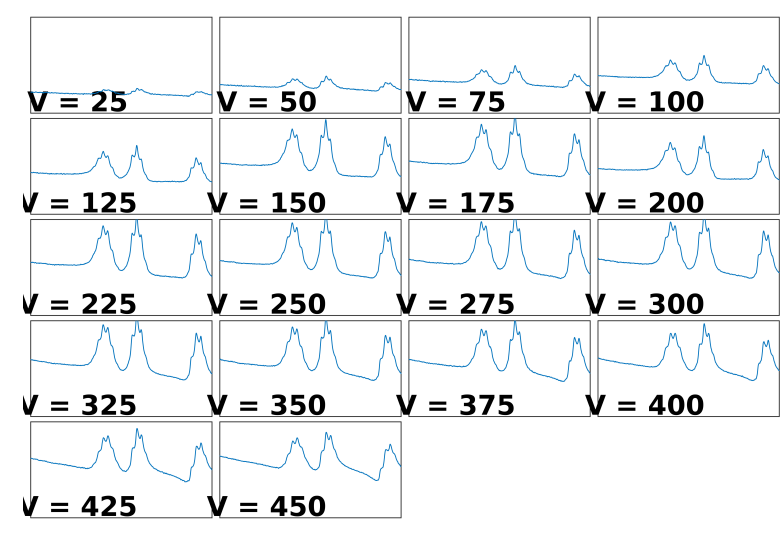

(a) Standard FID

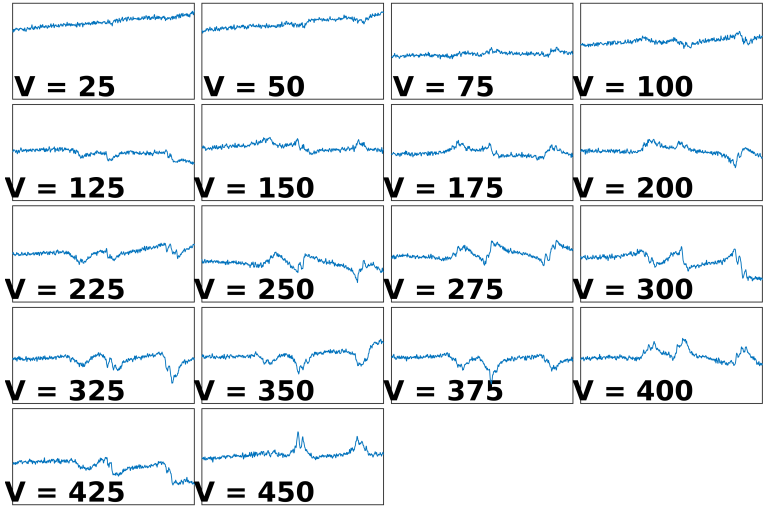

(b) Chemically selective pulse

Figure 6.9: Comparison of reference voltage calibration spectra for a standard FID, and an FID with a chemically selective pulse

Repeating this experiment, with the non-selective pulses replaced with the new optimised pulses produces very different results. For low transmitter voltages, the spectra produce no peaks, aside from some baseline. As the voltage is increased, some peaks begin to emerge, 
and the spectral profile of GABA is changed as the voltage is increased further. In particular, around $450 \mathrm{~V}$, a distinct two-peak pattern emerges, with the 3 PPM GABA resonance beginning to be suppressed. Unfortunately, at this point, the transmitter prevented further increases in the voltage due to the hardware constraints of our scanner.

The maximum transmitter voltage is limited to 1.2 times the reference voltage; the voltage required to achieve a $\pi$ rotation of the water signal, using a $1 \mathrm{~ms}$ non-selective, rectangular pulse. This is determined automatically by the scanner after phantom placement, using the previously mentioned calibration sequence. While it is possible to overwrite the reference voltage, doing so results in failure of all other pulses whose amplitudes are linked to this reference voltage, and therefore loss of water and outer volume suppression. This, too, could be circumvented by a sequence that performs a full manual calibration of the transmitter for all pulses, including water suppression. Implementation of this will be explored in future work. The results highlight the importance of hardware limitations, and the need to characterize these experimentally.

\subsubsection{Experimental $B_{1}$ mapping and calibration}

Even after the voltage has been calibrated, there will inevitably still be local $B_{1}$ field inhomogeneity. This results on different effective RF amplitudes across the extent of the sample. As we have seen, the conventional FID sequence is extremely robust with regard to such inhomogeneities, but optimal pulses will still be affected. To assess the degree of spatial inhomogeneity, $B_{1}$ field mapping can be performed. $B_{1}$ mapping was applied to a GABA phantom, and an oil phantom with two imaging planes. The sequence consists of a square excitation pulse of varying flip angle, $\alpha$, with frequency and phase encoding about a slice select refocussing pulse, as shown in Fig. 6.10. Fig. 6.11 shows a series of $B_{1}$ maps acquired for a variety of transmitter voltages. The contrast of the image lies in the distribution of effective flip angles in the sample, a feature which varies with transmitter voltage. Careful shimming and transmitter calibration can improve $B_{1}$ homogeneity, but it will always be a limiting factor in data quality, especially at high field strengths, where the effect is compounded. In the case of optimal pulses, selectivity will be destroyed by broad $B_{1}$ distributions, and previously suppressed signals will begin to emerge. 


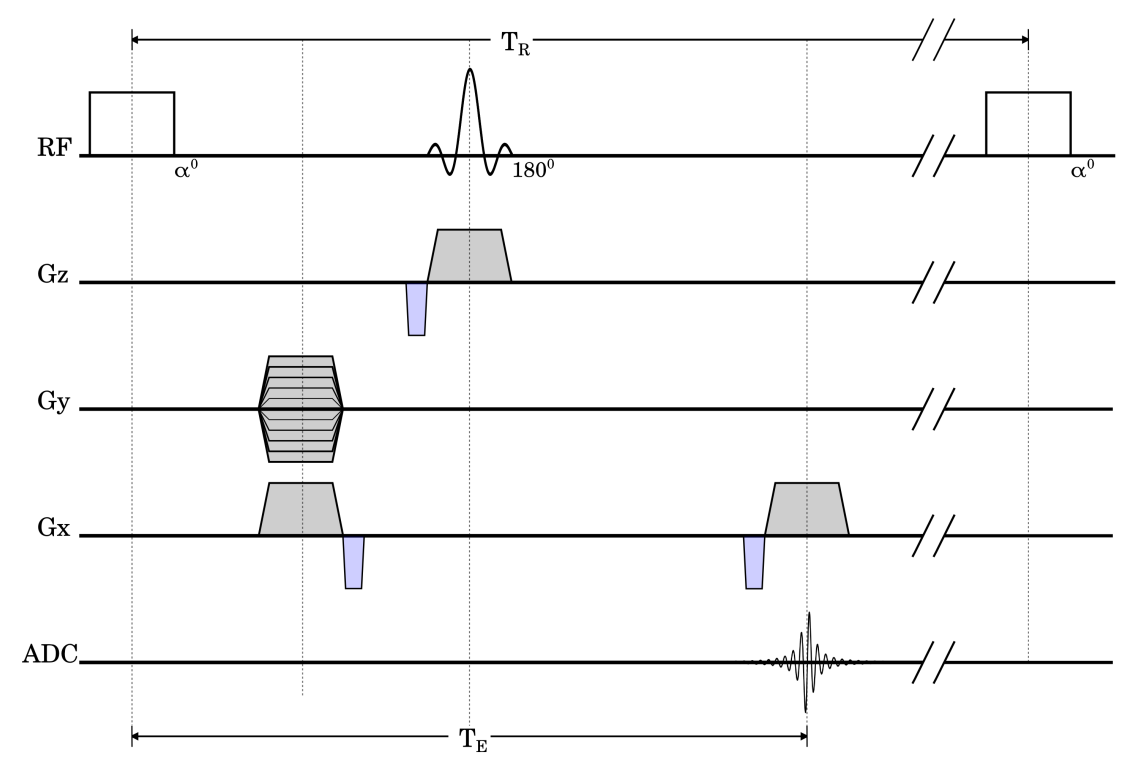

Figure 6.10: $B_{1}$ mapping sequence diagram.

Through localisation, it is possible restrict the excited volume, and therefore reduce the effects of $B_{1}$ spatial inhomogeneity in the final signal. However, the optimal RF pulses rely on complex amplitude and phase modulations, and the ability of the transmitter to accurately reproduce them. For conventional pulses, this process is not normally required, where the performance of the pulse can essentially be defined by the voltage calibration. There is, however a Siemens service sequence able to directly measure the waveforms produced by the transmitter, using the built-in RF receive coils. Fig. 6.13, and Fig. 6.12 show the temporal pulse profiles for a set of sinc, and rectangular pulses, respectively. The profiles allow visualisation of the transmitted pulses, over their duration, and multiple acquisitions. This was done for a variety of pulse lengths, and some interesting effects are observed. Short pulses appear unable to return to the zero baseline signal, creating a residual tail in the profiles. This is observed for both sinc and rectangular pulses. There is also a significant discrepancy between the two receive channels. While channel two is able to produce reasonable results for the longer pulses, the first channel consistently produces a dip in the centre. This kind of imperfect $B_{1}$ profile is an issue that needs to be addressed for the optimised RF pulses to work. 


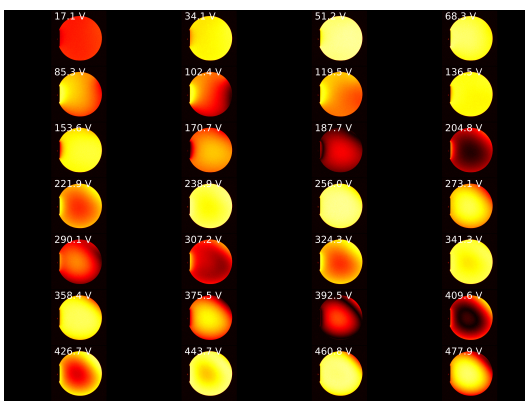

(a) Oil phantom - Transverse

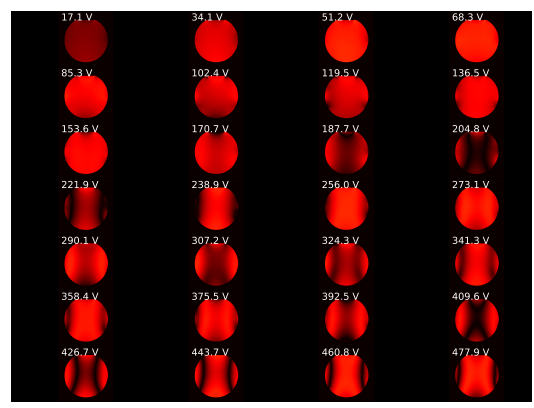

(b) Oil phantom - Sagital

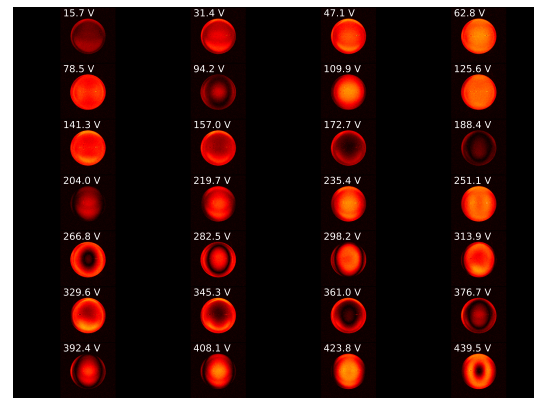

(c) GABA phantom

Figure 6.11: $B_{1}$ field maps for an oil, and GABA phantom. Contrast reflects differences in flip angles resulting from $B_{1}$ inhomogeneity.

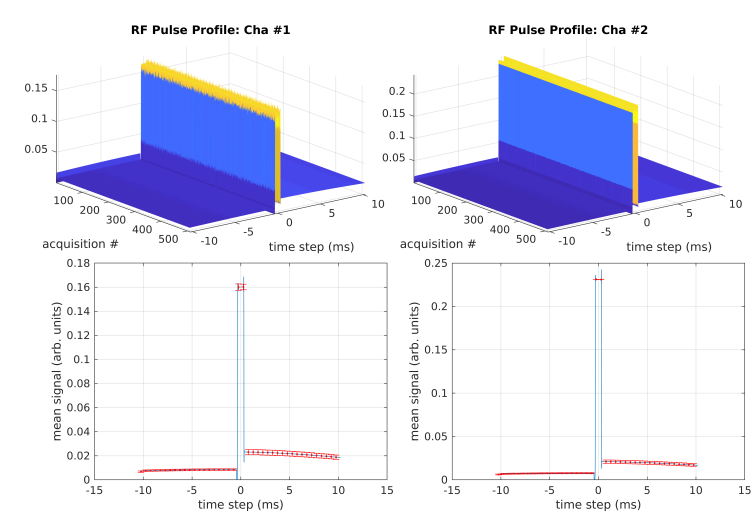

(a)

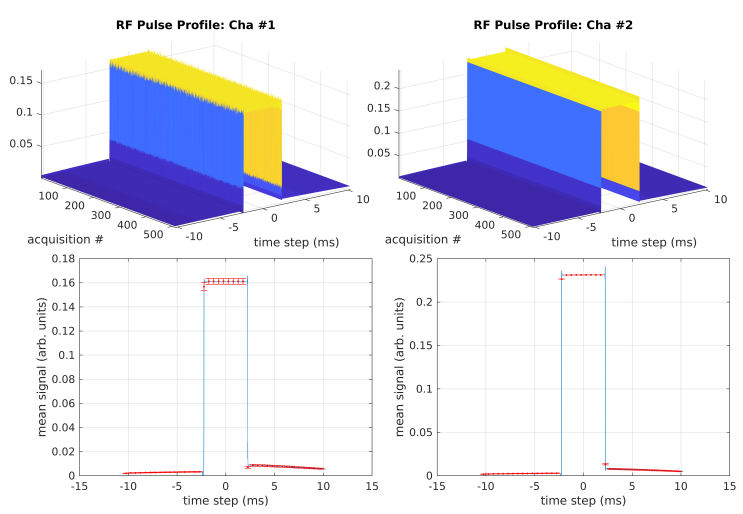

(c)

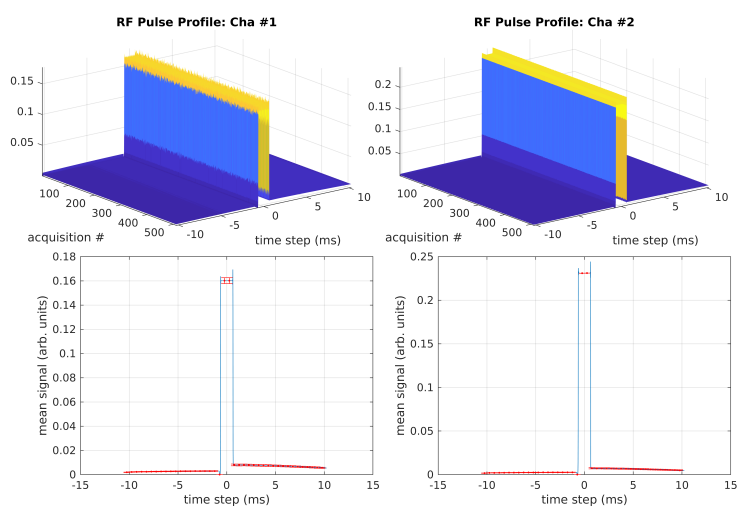

(b)

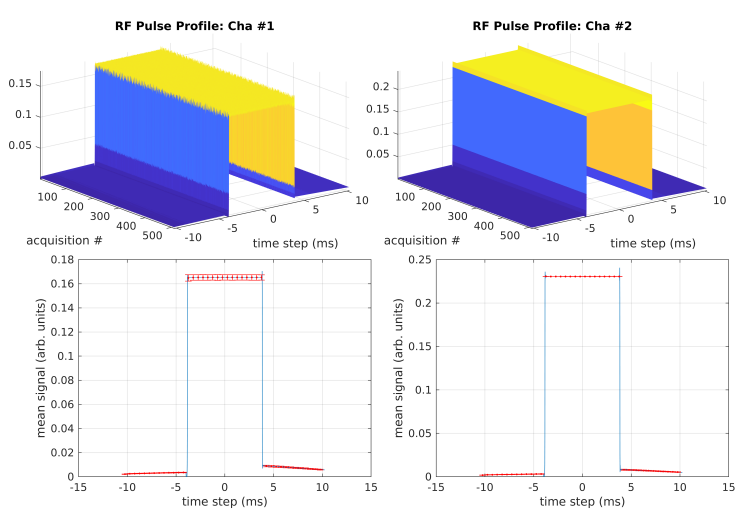

(d)

Figure 6.12: Rectangular pulse 


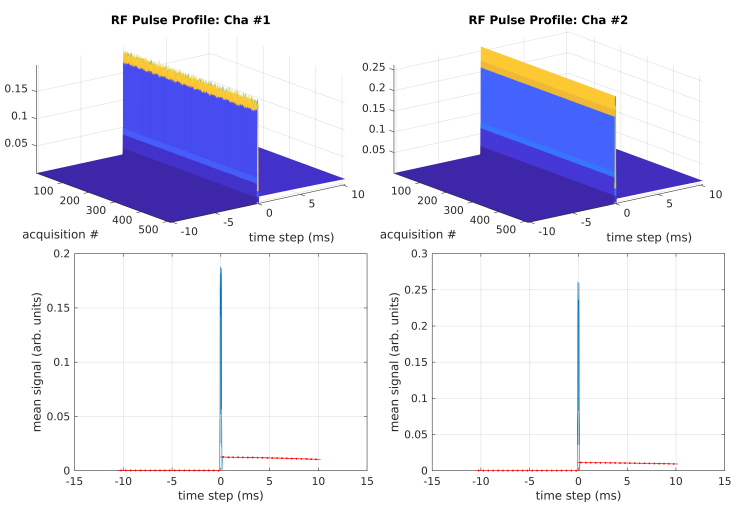

(a)

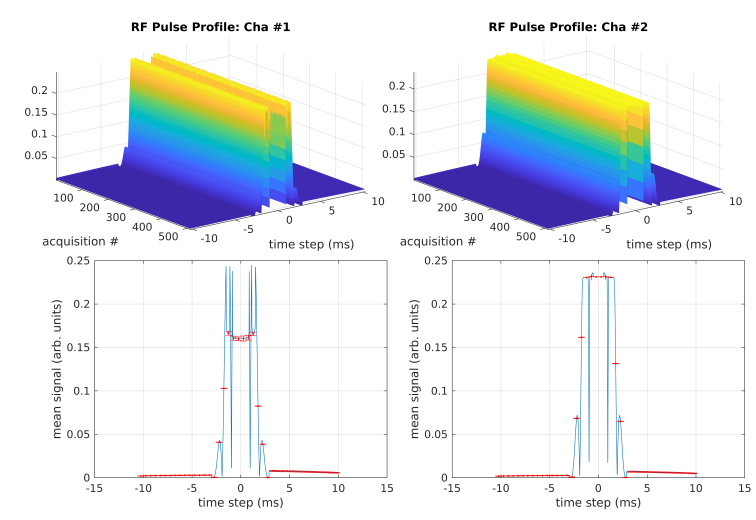

(c)

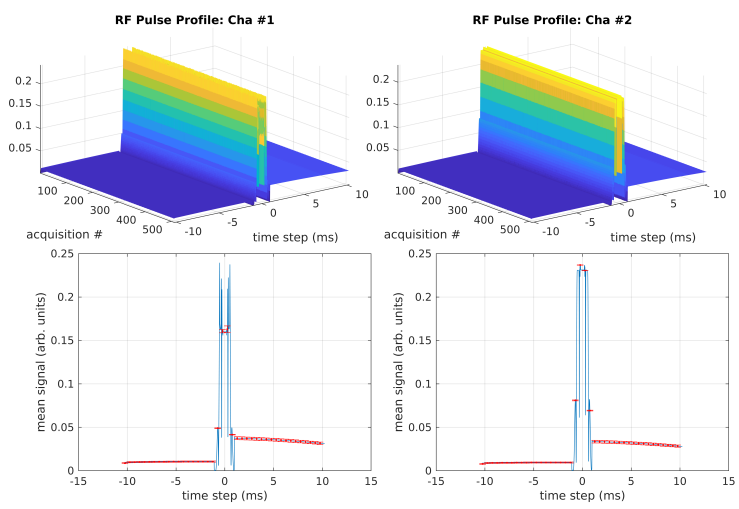

(b)

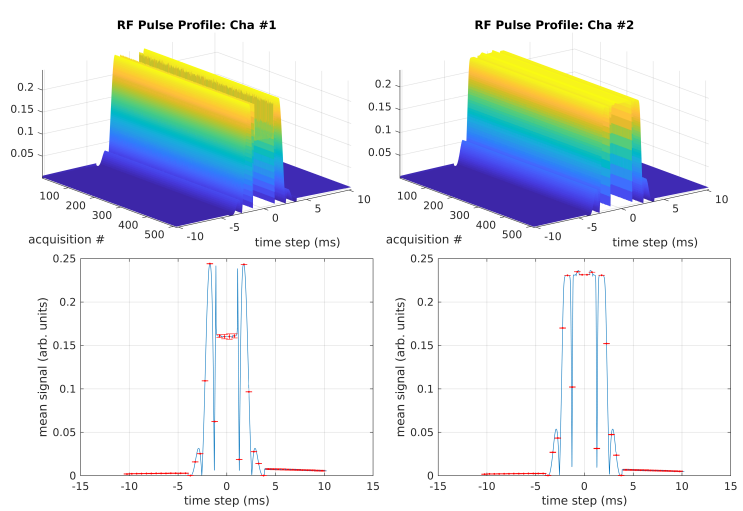

(d)

Figure 6.13: Sinc pulse

\subsection{Conclusion}

Pulses have been identified by the optimisation framework that allow discrimination of previously indiscernible spectroscopic signals. Simulations demonstrate a robustness of the pulses with respect to $B_{0}$ and $B_{1}$ inhomogeneities, and relaxation. The success of these pulses is dependent upon the accuracy of the underlying models. As seen in the previous chapter, there is variation in the reported chemical shifts and J-couplings. While these differences appear superficial in quantification, they are of greater importance in the optimisation, as the small model differences are the basis of manipulation. The validity of the models needs to be assessed, and the errors quantified. Model uncertainty could then be included into the optimisation process, enhancing the performance of pulses. Localisation remains problematic. 
Standard schemes destroying chemical selectivity, so signal localisation must be incorporated into the simulation, at significant computational cost, or outer volume suppression must be used.

Investigation into the calibration of the RF pulses has presented several pertinent questions. Can the scanner software produce the correct pulse shape? Is this shape damaged during amplification? Is the transmission fidelity high enough? Assessing each stage of this pipeline is important to ascertain the origin of any experimental errors. As future work, we plan to investigate this using an oscilloscope to measure the pre-amplified RF output of the scanner, and a Gauss probe to measure the actual field being created in the bore. Characterisation of any experimental issues here will need to be addressed before any work can continue. Conversely, if both of these stages produce reasonable results, then the model and simulation can be considered as the primary source of error, and the focus of further study. Successful experimental implication of chemically selective pulses would be a significant result. It could provide an alternative to spectral editing and two-dimensional techniques for in-vivo spectroscopy. The generality of the technique will allow application to many other target states and metabolites, and refinement of the process towards in-vivo use. 


\section{Conclusion}

The results presented in this thesis clearly show that MRI/S has considerable potential as a quantitative imaging technique, and as a tool for quantification of biomarkers. However, the pulse sequences, protocols, and analysis methods investigated in this thesis appear inadequate to access the full extent of available information. The initial study conducted into the calibration of MRI test objects served as a solid foundation for much of the subsequent experimental work. The well characterised, stable phantoms were a reliable baseline, and allowed the establishment of a ground truth with which to compare results. Calibrated relaxometry and spectroscopy studies are not possible in-vivo, the work conducted here would not have been nearly as informative without the ground truth phantom results. Our results suggest agarose as the most stable gelling agent, with $R^{2}$ of 0.96 , and 0.99 for $r_{1}$ and $r_{2}$ fits, respectively. While the agar results aren't as convincing, it is suggested as a cheaper alternative to agarose.

The comparative analysis of $R_{1}$ mapping techniques using both in-vivo data, and well characterized phantoms shows that there is considerable variability in the estimated $R_{1}$. In-vivo $R_{1}$ measurements show a considerable methodological dependence, with $\mathrm{SR}$ and IR t-test p-values ranging from $0-10^{-10}$. While the relaxivity fit quality, and temporal stability of $r_{1}$ estimates for a given sequence further vindicate the phantom preparation procedures, the variation of such a fundamental MR parameter by acquisition mode is a troubling finding for quantitative imaging. If the purported gold standard inversion recovery sequence is taken as a ground truth, other methods consistently over estimate longitudinal relaxivity. In the case of the variable flip angle methods, $r_{1}$ appears to be overestimated by around $60 \%$. This is an important finding for contrast enhanced MRI, where these fast $R_{1}$ mapping methods are used to quantify contrast agent uptake. Our results suggest a methodological dependence of relaxivity quantification methods, so comparing these fast mapping methods to inversion recovery maps, for instance, may not be best practice. DCE $R_{1}$ maps should ideally be compared to one acquired using the same sequence, to avoid introducing a systematic error. While the inconsistency of the various acquisition methods in the $R_{1}$ study is a concerning result, it does provide an interesting avenue of further study. The results appear to confirm 
inversion recovery as the gold standard technique, but the erroneous component of the other methods could still be present, even in these results. This, indeed, begs the question, what are we measuring? Further work is needed to fully investigate these issues, and until then, comparison of the measured $T_{1}$ should be restricted to specific acquisition methods to avoid any methodological dependencies.

Further complications are noted for spectroscopic techniques. Although in principle, the detection of biomarkers from unique spectroscopic signature should be easy, in practice it is far from straightforward, and there are many issues. Quantification is hampered by the temperature dependent water shift, potential $\mathrm{pH}$ effects to spectra, peak broadening due to many underlying causes, phase errors, and also varying contributions from lipids and macromolecules in-vivo. Moreover, as seen in this thesis, the concentration ratios reported by different analysis tools can vary greatly. With the exception of Tarquin, all tools appear to underestimate the actual GABA to NAA ratio. Solution series gradient linearity is generally good, with $R^{2}$ rarely dropping below 0.85 across all analysis tools. However, this is not the case for the gel series, where $R^{2}$ is consistently lower, and with a broader distribution of values. This exhibits the drop in performance as a result of imperfect data. The broader peaks of the gel data are far more challenging to fit, especially for a fixed basis set. Conversely, a consistent finding is the relatively good performance of the basic peak integration methods in both gradient estimation, and linearity. The robustness of these simple methods is promising, and could be seen as an alternative approach for specialist techniques, for example the optimised RF spectra. These results illustrate the need for studies on phantoms with known composition to establish a ground truth. In-vivo data is often analysed in multiple tools, with the discrepancies explained in relation to fit error. As stated previously, these errors can often obscure the real picture, with misidentified signals being attributed to other basis sets. The phantom work conducted here was able to establish a ground truth with which to compare to, allowing further appraisal of the quantification tools. Furthermore, this data set could be used to benchmark new analysis methods against the existing tools, allowing a quantitative measure of the success of quantification.

The chemically selective RF pulses presented here may address some of these problems, but will also present new challenges of their own. Model accuracy, field homogeneity, es- 
pecially over large volumes and/or high field strengths, and perhaps most fundamentally, the limitations of the RF transmitter. Initial optimisation results have identified pulses able to enforce single resonance excitation for multiple models simultaneously. Despite stringent requirements with regard to the pulse's characteristics, and experimental robustness, RFpulses have been identified that are able to discriminate between molecules as similar as Glutamate and glutamine. This is not commonly possible at 3T, so is an exciting prospective result. Attempts experimentally verify the pulses have highlighted the need for careful calibration, especially with regard to transmitter voltage.

The models of metabolites are fundamental in the final two chapters of this thesis, forming the basis of MRS analysis software, and the dynamics of the simulator. While the effect of basis appears to be small in the analysis, the relative simplicity of the GABA and NAA MEGA-PRESS spectra is perhaps not the sternest test. Complete analysis of conventional PRESS or STEAM spectra will require more accurate quantification of the molecular structures underlying the basis sets. For the RF optimisation work, these parameters are far more fundamental. When trying to find pulses that can discriminate between similar molecules, for example glutamine and glutamate, the minor differences become the sources of selectivity, so the model accuracy is paramount. However, our simulated results suggest that current pulses exhibit a relatively high robustness with respect to model variation, which bodes well for future work. Quantification of the models could even be an avenue of future work. High concentration metabolite phantoms could be made, and scanned over a range of sequence timings to elucidate the couplings and chemical shifts. These results could be utilised in both the analysis and optimisation work. One encouraging finding of the MRS quantification work is the relatively high quality of the peak integration methods. The commonly used MRS software would not be applicable to optimised spectra, without the generation of a specific basis set. The better option may be the simple analysis, at least in the context of phantom experiments.

Overall, there is certainly potential for quantitative imaging methods to thrive within MRI. Current techniques in relaxometry and spectroscopy, while promising, lack the numerical robustness required to become purely quantitative techniques. Generally, there is a need for standardisation. Both MRS and relaxometry have a method-dependent component 
to their analyses, but show reasonable consistency for intra-method comparisons. Further work is required to address these inconsistencies, but in the mean time, standardisation of protocols and analysis is recommended. Only then could these methods become truly quantitative. Additionally, while the experimental implementation of chemically selective pulses may be some way off, the potential applications for them are both numerous, and exciting. 
Appendices 


\section{A Supplementary material for test object analysis}

\begin{tabular}{|c|c||c|c||c|c|}
\hline Gelling agent & Concentration & $R_{1}\left(\mathrm{~s}^{-1}\right)$ & $R^{2}$ & $R_{2}\left(\mathrm{~s}^{-1}\right)$ & $R^{2}$ \\
\hline \multirow{7}{*}{ Agar } & $1.0 \%$ & $0.362(0.342,0.383)$ & 0.9996 & $6.282(6.357,6.208)$ & 0.9991 \\
& $1.5 \%$ & $0.389(0.365,0.414)$ & 0.9994 & $5.008(5.098,4.918)$ & 0.9980 \\
& $2.0 \%$ & $0.395(0.373,0.417)$ & 0.9995 & $11.275(11.657,10.892)$ & 0.9956 \\
& $2.5 \%$ & $0.409(0.387,0.432)$ & 0.9995 & $8.604(8.892,8.315)$ & 0.9960 \\
& $3.0 \%$ & $0.414(0.398,0.431)$ & 0.9997 & $9.956(10.383,9.529)$ & 0.9940 \\
& $3.5 \%$ & $0.469(0.456,0.482)$ & 0.9999 & $12.675(13.059,12.290)$ & 0.9953 \\
\hline \multirow{5}{*}{ Agarose } & $0.5 \%$ & $0.348(0.330,0.367)$ & 0.9996 & $4.911(4.950,4.872)$ & 0.9996 \\
& $1.0 \%$ & $0.351(0.332,0.370)$ & 0.9996 & $8.534(8.689,8.379)$ & 0.9982 \\
& $1.5 \%$ & $0.367(0.345,0.390)$ & 0.9995 & $12.037(12.512,11.562)$ & 0.9933 \\
& $2.0 \%$ & $0.385(0.353,0.418)$ & 0.9989 & $15.609(16.655,14.564)$ & 0.9820 \\
& $2.5 \%$ & $0.391(0.363,0.419)$ & 0.9992 & $18.495(20.310,16.680)$ & 0.9715 \\
& $3.0 \%$ & $0.399(0.375,0.422)$ & 0.9995 & $21.261(24.158,18.363)$ & 0.9639 \\
\hline \multirow{5}{*}{ PVA } & $10 \%$ & $0.690(0.686,0.694)$ & 1.0000 & $6.141(6.183,6.099)$ & 0.9997 \\
& $15 \%$ & $0.892(0.883,0.901)$ & 1.0000 & $11.130(11.329,10.932)$ & 0.9983 \\
& $20 \%$ & $1.069(1.058,1.081)$ & 1.0000 & $13.417(13.826,13.009)$ & 0.9965 \\
\hline
\end{tabular}

Table A.1: $R_{1}$ and $R_{2}$ values obtained from fitting, with $95 \%$ confidence interval, and $R^{2}$ fit error. 


\begin{tabular}{|c|c|c|c|}
\hline & Gelling agent & $R_{2}-2016$ & $R_{2}-2017$ \\
\hline \multirow{7}{*}{ Agar } & $1.0 \%$ & $6.282(6.357,6.208)$ & $6.25(6.15,6.35)$ \\
& $1.5 \%$ & $5.008(5.098,4.918)$ & $7.46(7.35,7.58)$ \\
& $2.0 \%$ & $11.275(11.657,10.892)$ & $11.54(11.24,11.85)$ \\
& $2.5 \%$ & $8.604(8.892,8.315)$ & $8.34(8.158 .53)$ \\
& $3.0 \%$ & $9.956(10.383,9.529)$ & $10.36(10.09,10.64)$ \\
& $3.5 \%$ & $12.675(13.059,12.290)$ & $12.89(12.50,13.29)$ \\
\hline \multirow{7}{*}{ Agarose } & $0.5 \%$ & $4.911(4.950,4.872)$ & $4.98(4.89,5.16)$ \\
& $1.0 \%$ & $8.534(8.689,8.379)$ & $8.76(8.61,8.92)$ \\
& $1.5 \%$ & $12.037(12.512,11.562)$ & $12.33(11.97,12.68)$ \\
& $2.0 \%$ & $15.609(16.655,14.564)$ & $15.82(15.01,16.62)$ \\
& $2.5 \%$ & $18.495(20.310,16.680)$ & $19.81(18.14,21.48)$ \\
& $3.0 \%$ & $21.261(24.158,18.363)$ & $22.14(19.90,24.39)$ \\
\hline
\end{tabular}

Table A.2: $R_{2}$ quantification resulting from two scan session over six months apart. 


\begin{tabular}{|c|c|c|c|}
\hline Contrast agent & Concentration & $R_{1}\left(\mathrm{~s}^{-1}\right)$ & $R^{2}$ \\
\hline \multirow{8}{*}{$\mathrm{Gd}_{2} \mathrm{O}_{3}$ Sept 16} & $0.002759 \mathrm{mM}$ & $1.070(1.012,1.127)$ & 0.9990 \\
\hline & $0.005517 \mathrm{mM}$ & $1.533(1.414,1.651)$ & 0.9978 \\
\hline & $0.013793 \mathrm{mM}$ & $2.622(2.406,2.838)$ & 0.9971 \\
\hline & $0.027586 \mathrm{mM}$ & $9.084(8.973,9.195)$ & 0.9998 \\
\hline & $0.055173 \mathrm{mM}$ & $11.382(11.284,11.481)$ & 0.9999 \\
\hline & $0.082759 \mathrm{mM}$ & $13.249(12.702,13.796)$ & 0.9963 \\
\hline & $0.110346 \mathrm{mM}$ & $20.410(19.589,21.231)$ & 0.9931 \\
\hline & $0.137932 \mathrm{mM}$ & $30.825(29.194,32.456)$ & 0.9769 \\
\hline \multirow{8}{*}{$\mathrm{Gd}_{2} \mathrm{O}_{3} \operatorname{Jan} 18$} & $0.002759 \mathrm{mM}$ & $1.038(0.976,1.100)$ & 0.9989 \\
\hline & $0.005517 \mathrm{mM}$ & $1.285(1.205,1.365)$ & 0.9987 \\
\hline & $0.013793 \mathrm{mM}$ & $2.545(2.369,2.720)$ & 0.9980 \\
\hline & $0.027586 \mathrm{mM}$ & $8.411(8.232,8.591)$ & 0.9994 \\
\hline & $0.055173 \mathrm{mM}$ & $11.240(11.156,11.324)$ & 0.9999 \\
\hline & $0.082759 \mathrm{mM}$ & $13.139(12.551,13.728)$ & 0.9957 \\
\hline & $0.110346 \mathrm{mM}$ & $19.259(18.381,20.137)$ & 0.9921 \\
\hline & $0.137932 \mathrm{mM}$ & $30.791(28.835,32.747)$ & 0.9672 \\
\hline \multirow{7}{*}{$\mathrm{Gd}_{2} \mathrm{O}_{3} \operatorname{Jan} 18$} & $0.002759 \mathrm{mM}$ & $0.763(0.723,0.802)$ & 0.9994 \\
\hline & $0.005517 \mathrm{mM}$ & $1.249(1.159,1.339)$ & 0.9983 \\
\hline & $0.013793 \mathrm{mM}$ & $2.425(2.211,2.638)$ & 0.9968 \\
\hline & $0.027586 \mathrm{mM}$ & $7.825(7.612,8.038)$ & 0.9992 \\
\hline & $0.055173 \mathrm{mM}$ & $9.483(8.940,10.026)$ & 0.9955 \\
\hline & $0.082759 \mathrm{mM}$ & $13.294(12.767,13.820)$ & 0.9966 \\
\hline & $0.110346 \mathrm{mM}$ & $21.466(21.093,21.839)$ & 0.9986 \\
\hline
\end{tabular}

Table A.3: $R_{1}$ fits for $\mathrm{Gd}_{2} \mathrm{O}_{3}$ scans, with $95 \%$ convidence intervals, and $R^{2}$ values 


\begin{tabular}{|c|c||c|c|}
\hline Contrast agent & Concentration & $R_{1}\left(\mathrm{~s}^{-1}\right)$ & $R^{2}$ \\
\hline & $0.000000 \mathrm{mM}$ & $0.331(0.304,0.358)$ & 1.0000 \\
& $0.050528 \mathrm{mM}$ & $0.853(0.815,0.891)$ & 0.9999 \\
$\mathrm{MnCl}_{2}$ July 16 & $0.101056 \mathrm{mM}$ & $1.341(1.270,1.413)$ & 0.9998 \\
& $0.151584 \mathrm{mM}$ & $1.935(1.910,1.960)$ & 1.0000 \\
& $0.202112 \mathrm{mM}$ & $2.920(2.878,2.962)$ & 1.0000 \\
& $0.252640 \mathrm{mM}$ & $3.055(2.981,3.130)$ & 0.9999 \\
& $0.303168 \mathrm{mM}$ & $4.221(4.154,4.288)$ & 0.9999 \\
\hline \multirow{7}{*}{$\mathrm{MnCl}_{2}$ Nov 17 } & $0.000000 \mathrm{mM}$ & $0.363(0.329,0.396)$ & 0.9997 \\
& $0.050528 \mathrm{mM}$ & $1.045(0.992,1.098)$ & 0.9997 \\
& $0.101056 \mathrm{mM}$ & $1.249(1.085,1.413)$ & 0.9977 \\
& $0.202112 \mathrm{mM}$ & $2.626(2.546,2.705)$ & 0.9998 \\
& $0.252640 \mathrm{mM}$ & $2.967(2.717,3.217)$ & 0.9982 \\
& $0.303168 \mathrm{mM}$ & $4.112(3.795,4.429)$ & 0.9980 \\
\hline & $0.000000 \mathrm{mM}$ & $0.375(0.358,0.393)$ & 0.9998 \\
& $0.050528 \mathrm{mM}$ & $1.012(0.983,1.041)$ & 0.9998 \\
& $0.101056 \mathrm{mM}$ & $1.280(1.172,1.387)$ & 0.9982 \\
& $0.151584 \mathrm{mM}$ & $1.691(1.561,1.821)$ & 0.9982 \\
& $0.202112 \mathrm{mM}$ & $2.567(2.541,2.593)$ & 1.0000 \\
& $0.252640 \mathrm{mM}$ & $3.003(2.809,3.198)$ & 0.9980 \\
& $0.303168 \mathrm{mM}$ & $4.178(3.948,4.408)$ & 0.9980 \\
\hline
\end{tabular}

Table A.4: $R_{1}$ fits for $\mathrm{MnCl}_{2}$ scans, with $95 \%$ convidence intervals, and $R^{2}$ values 


\begin{tabular}{|c|c|c|c|}
\hline Contrast agent & Concentration & $R_{2}\left(\mathrm{~s}^{-1}\right)$ & $R^{2}$ \\
\hline \multirow{8}{*}{$\mathrm{Gd}_{2} \mathrm{O}_{3}$} & $0.002759 \mathrm{mM}$ & $10.136(10.245,10.027)$ & 0.9973 \\
\hline & $0.005517 \mathrm{mM}$ & $10.830(10.941,10.719)$ & 0.9977 \\
\hline & $0.013793 \mathrm{mM}$ & $13.115(13.255,12.975)$ & 0.9978 \\
\hline & $0.027586 \mathrm{mM}$ & $19.161(19.389,18.933)$ & 0.9985 \\
\hline & $0.055173 \mathrm{mM}$ & $22.810(23.157,22.462)$ & 0.9983 \\
\hline & $0.082759 \mathrm{mM}$ & $27.850(28.317,27.382)$ & 0.9981 \\
\hline & $0.110346 \mathrm{mM}$ & $35.802(36.669,34.935)$ & 0.9970 \\
\hline & $0.137932 \mathrm{mM}$ & $47.156(48.430,45.881)$ & 0.9968 \\
\hline \multirow{7}{*}{$\mathrm{MnCl}_{2}$ July 16} & $0.000000 \mathrm{mM}$ & $7.061(7.139,6.982)$ & 0.9992 \\
\hline & $0.050528 \mathrm{mM}$ & $12.986(13.464,12.508)$ & 0.9928 \\
\hline & $0.101056 \mathrm{mM}$ & $19.230(20.789,17.672)$ & 0.9772 \\
\hline & $0.151584 \mathrm{mM}$ & $22.070(22.988,21.151)$ & 0.9952 \\
\hline & $0.202112 \mathrm{mM}$ & $26.439(27.844,25.034)$ & 0.9922 \\
\hline & $0.252640 \mathrm{mM}$ & $31.720(33.915,29.524)$ & 0.9904 \\
\hline & $0.303168 \mathrm{mM}$ & $40.536(45.184,35.888)$ & 0.9838 \\
\hline \multirow{7}{*}{$\mathrm{MnCl}_{2}$ Nov 17} & $0.000000 \mathrm{mM}$ & $7.817(7.937,7.696)$ & 0.9983 \\
\hline & $0.050528 \mathrm{mM}$ & $14.213(14.946,13.480)$ & 0.9852 \\
\hline & $0.101056 \mathrm{mM}$ & $18.931(20.211,17.651)$ & 0.9817 \\
\hline & $0.151584 \mathrm{mM}$ & $24.727(27.440,22.014)$ & 0.9705 \\
\hline & $0.202112 \mathrm{mM}$ & $31.349(37.881,24.818)$ & 0.9387 \\
\hline & $0.252640 \mathrm{mM}$ & $41.710(56.628,26.792)$ & 0.8864 \\
\hline & $0.303168 \mathrm{mM}$ & $52.987(82.688,23.286)$ & 0.8079 \\
\hline \multirow{7}{*}{$\mathrm{MnCl}_{2}$ Nov 17} & $0.000000 \mathrm{mM}$ & $7.791(7.909,7.672)$ & 0.9983 \\
\hline & $0.050528 \mathrm{mM}$ & $14.395(15.258,13.533)$ & 0.9784 \\
\hline & $0.101056 \mathrm{mM}$ & $18.993(20.313,17.674)$ & 0.9807 \\
\hline & $0.151584 \mathrm{mM}$ & $25.862(29.514,22.209)$ & 0.9428 \\
\hline & $0.202112 \mathrm{mM}$ & $31.562(38.451,24.674)$ & 0.9331 \\
\hline & $0.252640 \mathrm{mM}$ & $41.453(54.457,28.450)$ & 0.8711 \\
\hline & $0.303168 \mathrm{mM}$ & $57.390(100.832,13.947)$ & 0.7708 \\
\hline
\end{tabular}

Table A.5: $R_{2}$ fits for both $\mathrm{Gd}_{2} \mathrm{O}_{3}$ and $\mathrm{MnCl}_{2}$ scans, with $95 \%$ convidence intervals, and $R^{2}$ values. 
B Supplementary material for relaxometry 

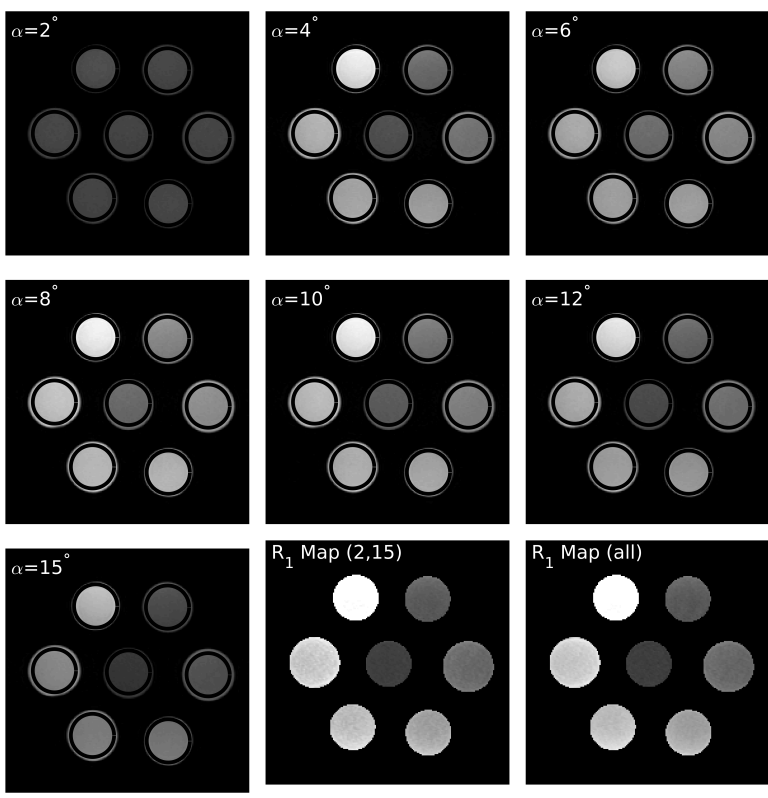

Figure B.1: VFA series images with $R_{1}$ maps obtained using only two flip angles (2 and 15 degrees) and 9 flip angles $\left(2,3,4,6,8,10,12,13,15\right.$ degrees) for $\mathrm{MnCl}_{2}$ gels. 

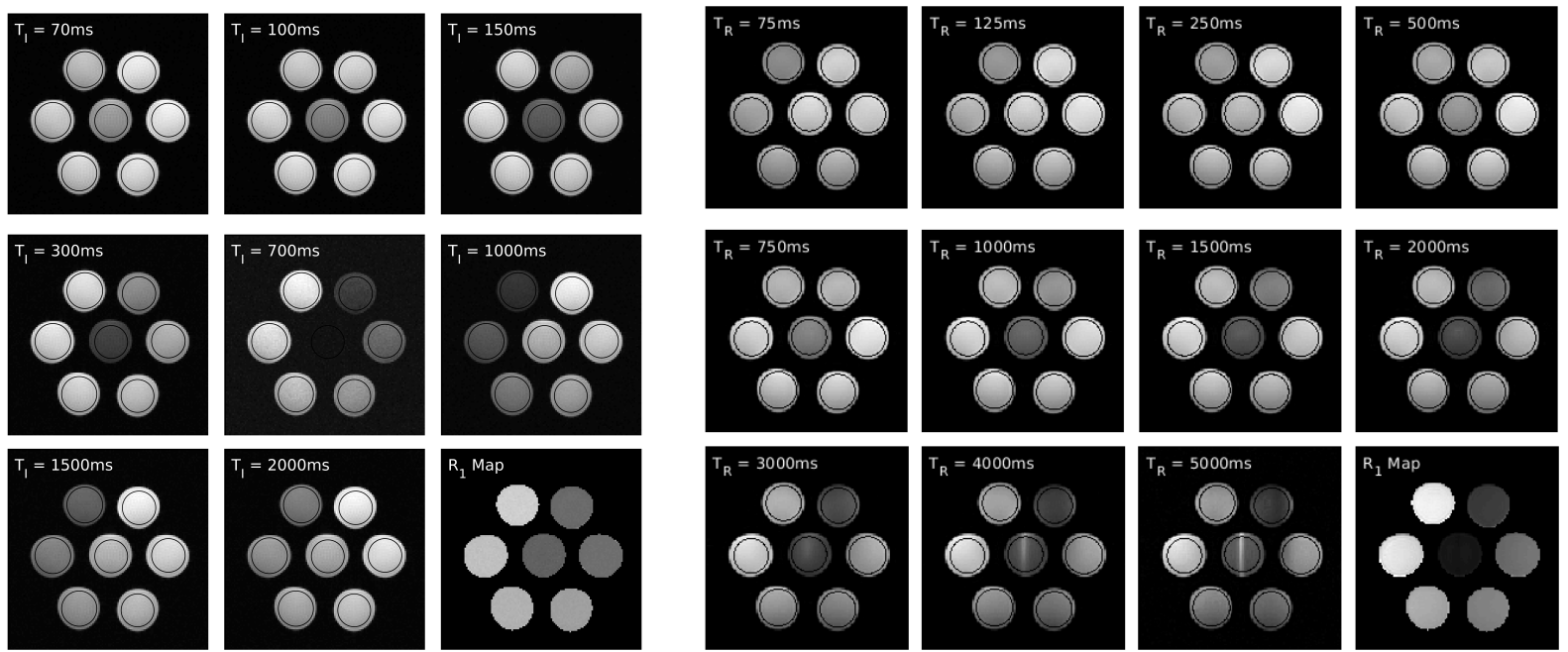

(a) IR Series

(b) SR Series

Figure B.2: Inversion recovery and saturation recovery images for $\mathrm{MnCl}_{2}$ solutions.

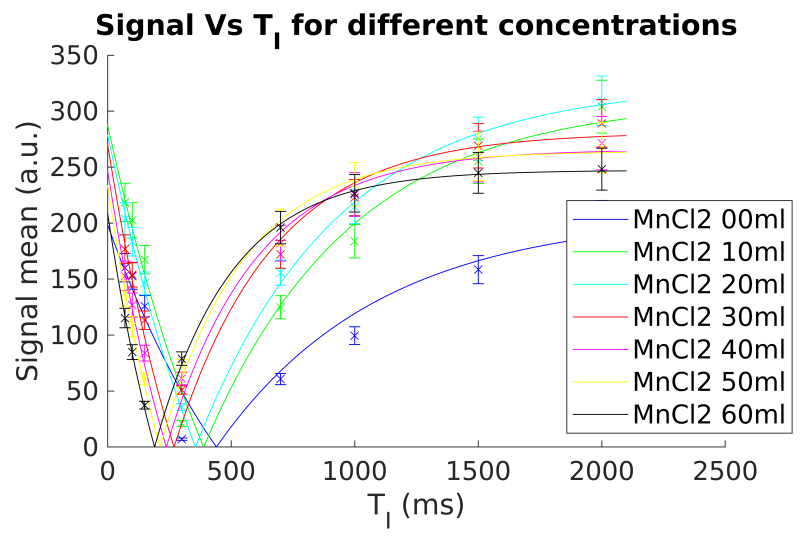

(a) IR Graphs

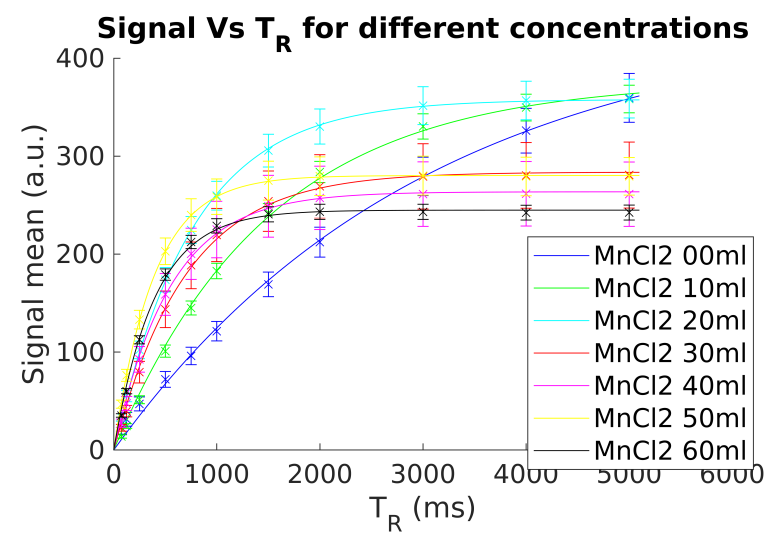

(b) SR Graphs

Figure B.3: Mean signal vs $T_{I}\left(T_{R}\right)$ graphs for $\mathrm{MnCl}_{2}$ contrast solutions. The error bars indicate the variation of the signal over the ROI. The solid lines are the curves of best fit. 

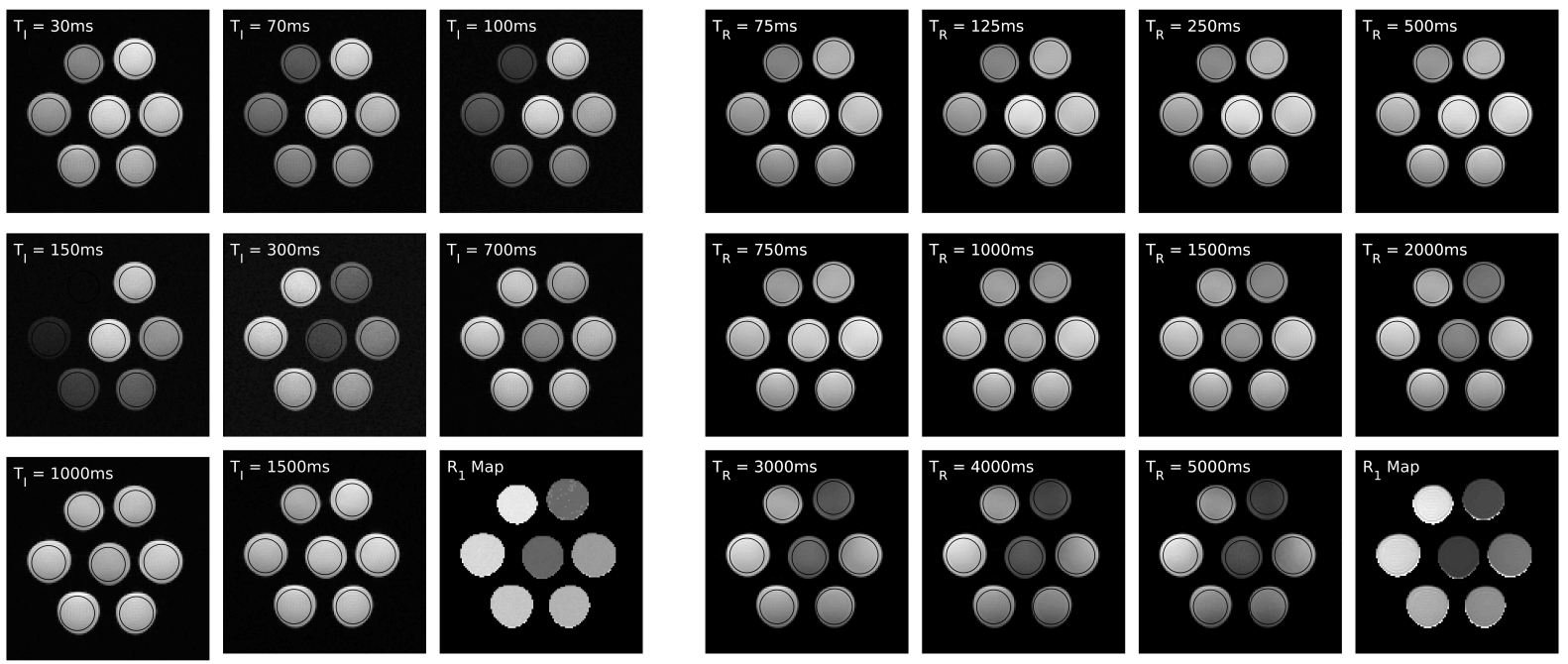

(a) IR Series

(b) SR Series

Figure B.4: Inversion recovery and saturation recovery images for $\mathrm{MnCl}_{2}$ gel phantoms.

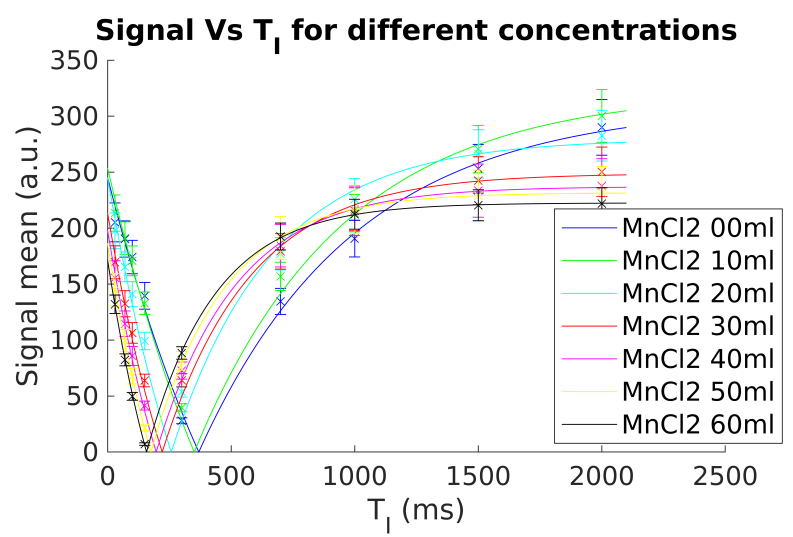

(a) IR Graphs

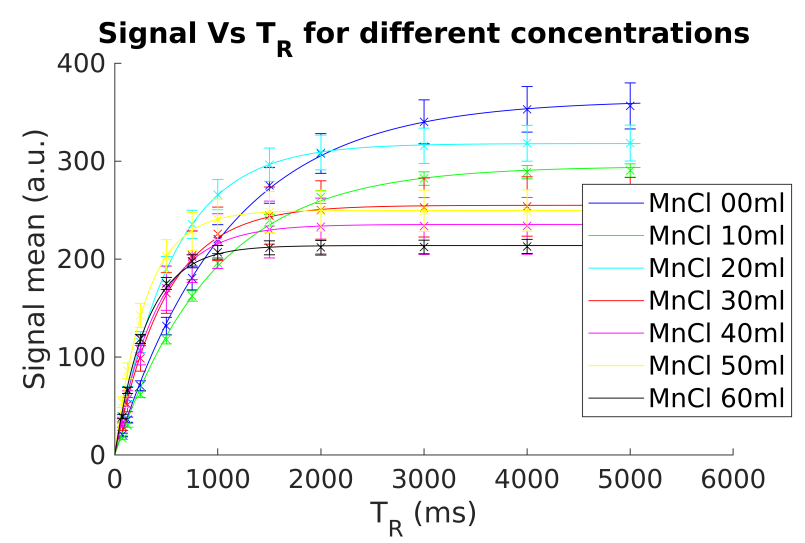

(b) SR Graphs

Figure B.5: Mean signal vs $T_{I}\left(T_{R}\right)$ graphs for $\mathrm{MnCl}_{2}$ contrast gel phantoms. The error bars indicate the variation of the signal over the ROI. The solid lines are the curves of best fit. 


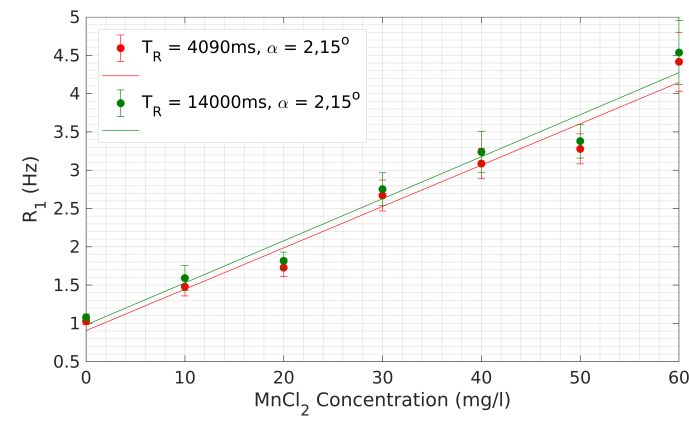

(a) 2-point methods with different $T_{R}$

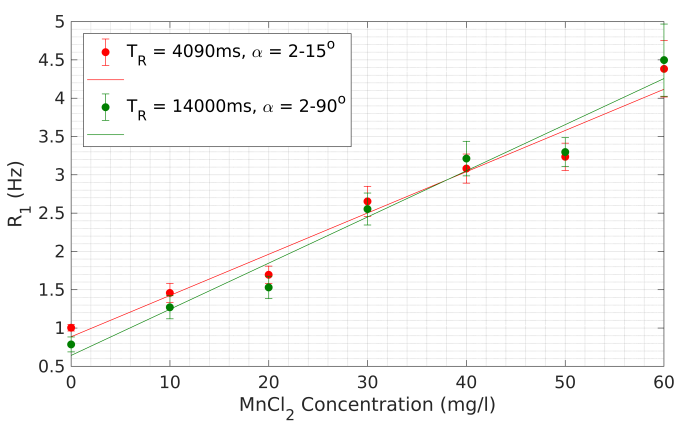

(b) 9-point(red) and 12-point(green) relaxivity curves for $\mathrm{MnCl}_{2}$ gels

Figure B.6: Relaxivity of $\mathrm{MnCl}_{2}$ gel phantoms for different VFA methods 


\section{Tables of chemical shifts and coupling constants for various metabolites.}

\begin{tabular}{|l||c|c|c|c|c|c|}
\hline $\mathbf{V}(\mathbf{P P M})$ & $J_{1}(\mathrm{~Hz})$ & $J_{2}(\mathrm{~Hz})$ & $J_{3}(\mathrm{~Hz})$ & $J_{4}(\mathrm{~Hz})$ & $J_{5}(\mathrm{~Hz})$ & $J_{6}(\mathrm{~Hz})$ \\
\hline 6.649 & 0 & 0 & 0 & 0 & 0 & 0 \\
3.027 & 0 & 0 & 0 & 0 & 0 & 0 \\
3.027 & 0 & 0 & 0 & 0 & 0 & 0 \\
3.027 & 0 & 0 & 0 & 0 & 0 & 0 \\
3.913 & 0 & 0 & 0 & 0 & 0 & 0 \\
3.913 & 0 & 0 & 0 & 0 & 0 & 0 \\
\hline
\end{tabular}

Table C.1: Chemical shifts and scalar couplings of Creatine [1]. Notice the zero-valued coupling matrix. Creatine is a set of 3 uncoupled resonances.

\begin{tabular}{|c||c|c|c|c|c|c|}
\hline $\mathbf{V}(\mathbf{P P M})$ & $J_{1}(\mathrm{~Hz})$ & $J_{2}(\mathrm{~Hz})$ & $J_{3}(\mathrm{~Hz})$ & $J_{4}(\mathrm{~Hz})$ & $J_{5}(\mathrm{~Hz})$ & $J_{6}(\mathrm{~Hz})$ \\
\hline 3.0128 & 0 & 0 & 5.372 & 7.127 & 0 & 0 \\
3.0128 & 0 & 0 & 10.578 & 6.982 & 0 & 0 \\
1.889 & 5.372 & 10.578 & 0 & 0 & 7.755 & 7.432 \\
1.889 & 7.127 & 6.982 & 0 & 0 & 6.173 & 7.933 \\
2.284 & 0 & 0 & 7.755 & 6.173 & 0 & 0 \\
2.284 & 0 & 0 & 7.432 & 7.933 & 0 & 0 \\
\hline
\end{tabular}

Table C.2: Chemical shifts and scalar couplings of Govindarau GABA model [1] 


\begin{tabular}{|c||c|c|c|c|c|c|}
\hline $\mathbf{V}(\mathbf{P P M})$ & $J_{1}(\mathrm{~Hz})$ & $J_{2}(\mathrm{~Hz})$ & $J_{3}(\mathrm{~Hz})$ & $J_{4}(\mathrm{~Hz})$ & $J_{5}(\mathrm{~Hz})$ & $J_{6}(\mathrm{~Hz})$ \\
\hline 2.284 & 0 & 0 & 7.352 & 7.352 & 0 & 0 \\
2.284 & 0 & 0 & 7.352 & 7.352 & 0 & 0 \\
1.888 & 7.352 & 7.352 & 0 & 0 & 6.377 & 7.96 \\
1.888 & 7.352 & 7.352 & 0 & 0 & 8.138 & 7.495 \\
3.012 & 0 & 0 & 6.377 & 8.138 & 0 & 0 \\
3.012 & 0 & 0 & 7.96 & 7.495 & 0 & 0 \\
\hline
\end{tabular}

Table C.3: Chemical shifts and scalar couplings of Kaiser GABA model [2]

\begin{tabular}{|c||c|c|c|c|c|c|}
\hline $\mathbf{V}(\mathbf{P P M})$ & $J_{1}(\mathrm{~Hz})$ & $J_{2}(\mathrm{~Hz})$ & $J_{3}(\mathrm{~Hz})$ & $J_{4}(\mathrm{~Hz})$ & $J_{5}(\mathrm{~Hz})$ & $J_{6}(\mathrm{~Hz})$ \\
\hline 2.284 & 0 & -15.938 & 7.678 & 6.98 & 0 & 0 \\
2.284 & -15.938 & 0 & 6.98 & 7.678 & 0 & 0 \\
1.888 & 7.678 & 6.98 & 0 & -15 & 8.51 & 6.503 \\
1.888 & 6.98 & 7.678 & -15 & 0 & 6.503 & 8.51 \\
3.013 & 0 & 0 & 8.51 & 6.503 & 0 & -14.062 \\
3.013 & 0 & 0 & 6.503 & 8.51 & -14.062 & 0 \\
\hline
\end{tabular}

Table C.4: Chemical shifts and scalar couplings of Near GABA model [3]

\begin{tabular}{|c||c|c|c|c|c|}
\hline $\mathbf{V}(\mathbf{P P M})$ & $J_{1}(\mathrm{~Hz})$ & $J_{2}(\mathrm{~Hz})$ & $J_{3}(\mathrm{~Hz})$ & $J_{4}(\mathrm{~Hz})$ & $J_{5}(\mathrm{~Hz})$ \\
\hline 3.7433 & 0 & 7.331 & 4.651 & 0 & 0 \\
2.0375 & 7.331 & 0 & -14.848 & 6.413 & 8.406 \\
2.12 & 4.651 & -14.848 & 0 & 8.478 & 6.875 \\
2.3378 & 0 & 6.413 & 8.478 & 0 & -15.915 \\
2.352 & 0 & 8.406 & 6.875 & -15.915 & 0 \\
\hline
\end{tabular}

Table C.5: Chemical shifts and scalar couplings of Glutamate [1]. 


\begin{tabular}{|c||c|c|c|c|c|}
\hline $\mathbf{V}(\mathbf{P P M})$ & $J_{1}(\mathrm{~Hz})$ & $J_{2}(\mathrm{~Hz})$ & $J_{3}(\mathrm{~Hz})$ & $J_{4}(\mathrm{~Hz})$ & $J_{5}(\mathrm{~Hz})$ \\
\hline 3.753 & 0 & 5.847 & 6.5 & 0 & 0 \\
2.129 & 5.847 & 0 & -14.504 & 9.165 & 6.347 \\
2.109 & 6.5 & -14.504 & 0 & 6.324 & 9.209 \\
2.432 & 0 & 9.165 & 6.324 & 0 & -15.371 \\
2.454 & 0 & 6.347 & 9.209 & -15.371 & 0 \\
\hline
\end{tabular}

Table C.6: Chemical shifts and scalar couplings of Glutamine [1].

\begin{tabular}{|c||c|c|}
\hline $\mathbf{V}(\mathbf{P P M})$ & $J_{1}(\mathrm{~Hz})$ & $J_{2}(\mathrm{~Hz})$ \\
\hline 3.548 & 0 & 0 \\
3.548 & 0 & 0 \\
\hline
\end{tabular}

Table C.7: Chemical shifts and scalar couplings of Glycine [1].

\begin{tabular}{|c||c|c|c|c|}
\hline $\mathbf{V}(\mathbf{P P M})$ & $J_{1}(\mathrm{~Hz})$ & $J_{2}(\mathrm{~Hz})$ & $J_{3}(\mathrm{~Hz})$ & $J_{4}(\mathrm{~Hz})$ \\
\hline 4.0974 & 0 & 6.933 & 6.933 & 6.933 \\
1.3142 & 6.933 & 0 & 0 & 0 \\
1.3142 & 6.933 & 0 & 0 & 0 \\
1.3142 & 6.933 & 0 & 0 & 0 \\
\hline
\end{tabular}

Table C.8: Chemical shifts and scalar couplings of Lactate [1]

\begin{tabular}{|c||c|c|c|c|c|c|}
\hline $\mathbf{V}(\mathbf{P P M})$ & $J_{1}(\mathrm{~Hz})$ & $J_{2}(\mathrm{~Hz})$ & $J_{3}(\mathrm{~Hz})$ & $J_{4}(\mathrm{~Hz})$ & $J_{5}(\mathrm{~Hz})$ & $J_{6}(\mathrm{~Hz})$ \\
\hline 3.5217 & 0 & 2.889 & 0 & 0 & 0 & 9.998 \\
4.0538 & 2.889 & 0 & 3.006 & 0 & 0 & 0 \\
3.5217 & 0 & 3.006 & 0 & 9.997 & 0 & 0 \\
3.6144 & 0 & 0 & 9.997 & 0 & 9.485 & 0 \\
3.269 & 0 & 0 & 0 & 9.485 & 0 & 9.482 \\
3.6144 & 9.998 & 0 & 0 & 0 & 9.482 & 0 \\
\hline
\end{tabular}

Table C.9: Chemical shifts and scalar couplings of myo-inositol [1]. 


\begin{tabular}{|c||c|c|c|c|c|c|c|}
\hline $\mathbf{V}(\mathbf{P P M})$ & $J_{1}(\mathrm{~Hz})$ & $J_{2}(\mathrm{~Hz})$ & $J_{3}(\mathrm{~Hz})$ & $J_{4}(\mathrm{~Hz})$ & $J_{5}(\mathrm{~Hz})$ & $J_{6}(\mathrm{~Hz})$ & $J_{7}(\mathrm{~Hz})$ \\
\hline 4.3817 & 0 & 3.861 & 3.861 & 6.4 & 0 & 0 & 0 \\
2.6727 & 3.861 & 0 & -15.592 & 0 & 3.861 & 3.861 & 3.861 \\
2.4863 & 3.861 & -15.592 & 0 & 0 & 3.861 & 3.861 & 3.861 \\
7.8205 & 6.4 & 0 & 0 & 0 & 6.4 & 6.4 & 6.4 \\
2.008 & 0 & 3.861 & 3.861 & 6.4 & 0 & 0 & 0 \\
2.008 & 0 & 3.861 & 3.861 & 6.4 & 0 & 0 & 0 \\
2.008 & 0 & 3.861 & 3.861 & 6.4 & 0 & 0 & 0 \\
\hline
\end{tabular}

Table C.10: Chemical shifts and scalar couplings of NAA [1]. 


\section{FID-A simulated results.}
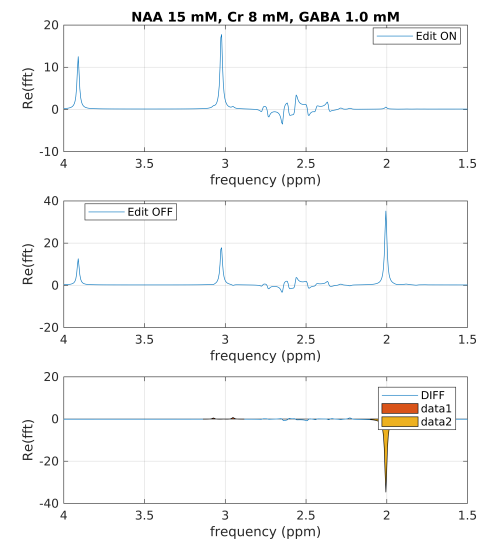

(a) $1.0 \mathrm{mmol}$
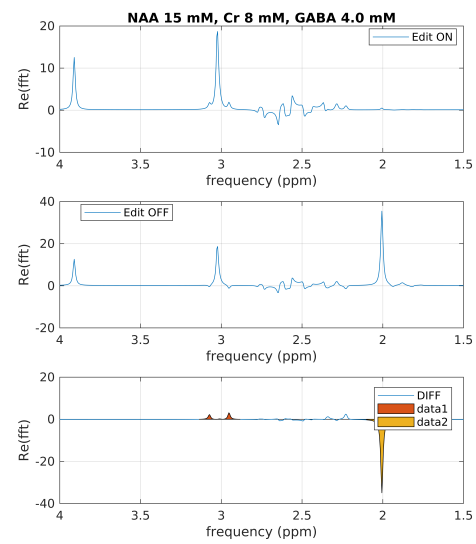

(d) $4.0 \mathrm{mmol}$
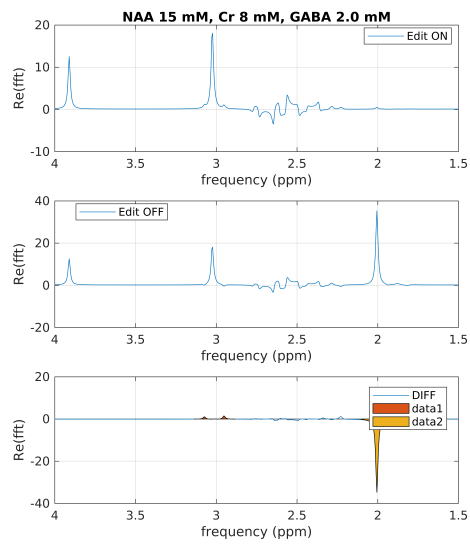

(b) $2.0 \mathrm{mmol}$
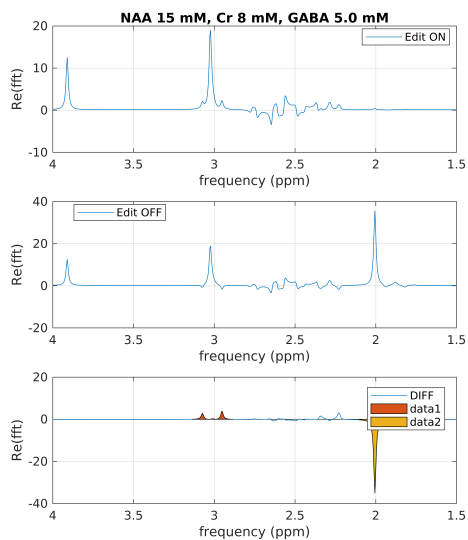

(e) $5.0 \mathrm{mmol}$
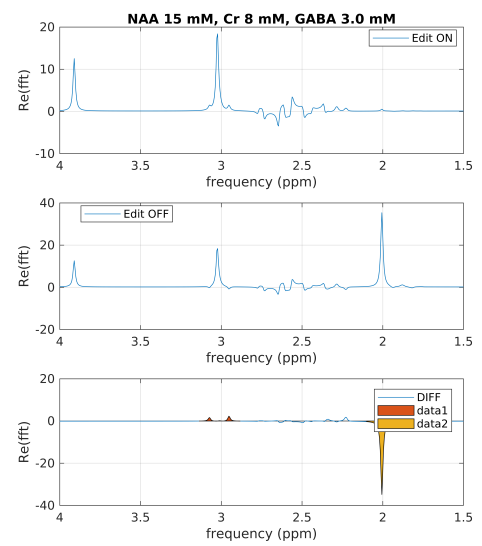

(c) $3.0 \mathrm{mmol}$
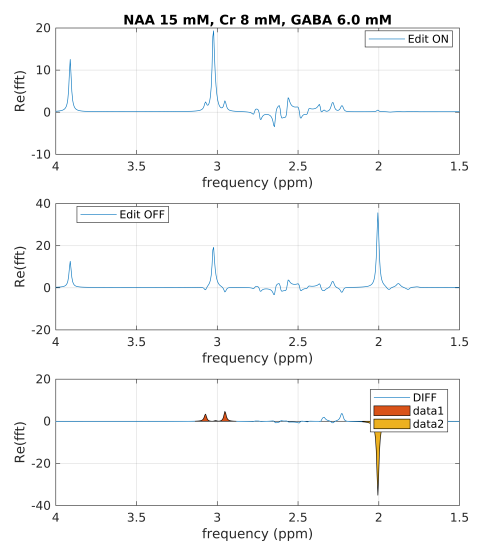

(f) $6.0 \mathrm{mmol}$

Figure D.1 

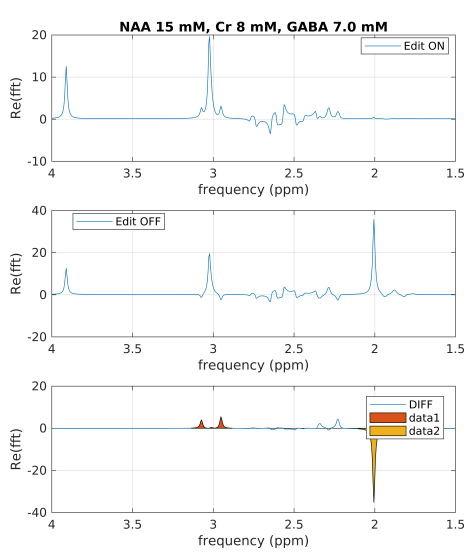

(a) $7.0 \mathrm{mmol}$
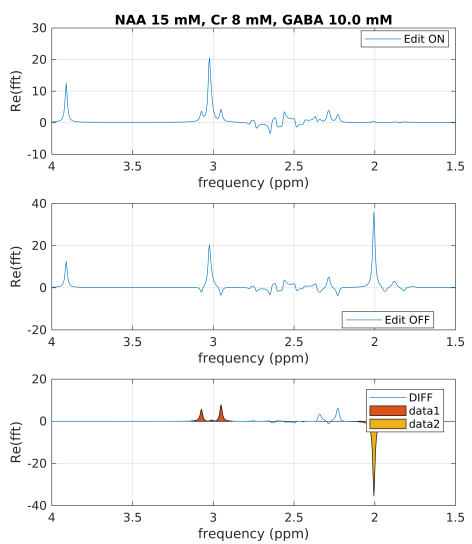

(d) $10.0 \mathrm{mmol}$
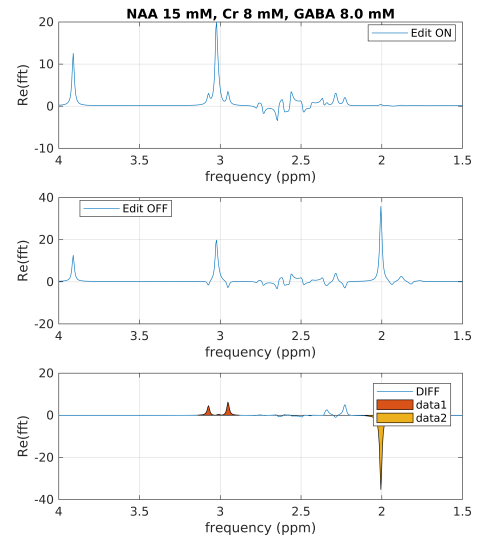

(b) $8.0 \mathrm{mmol}$
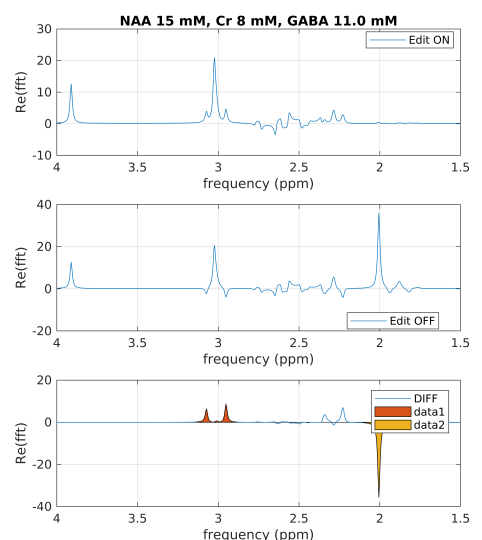

(e) $11.0 \mathrm{mmol}$
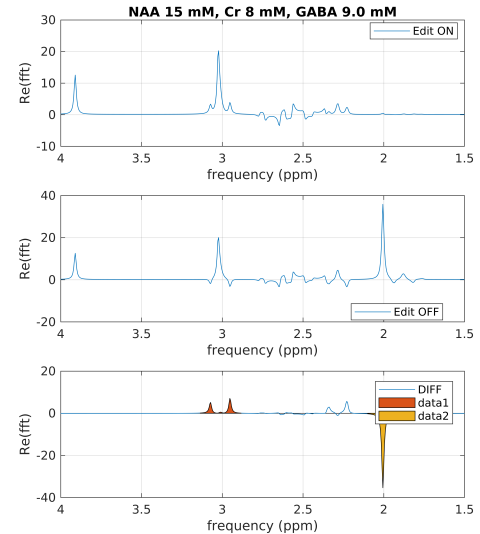

(c) $9.0 \mathrm{mmol}$
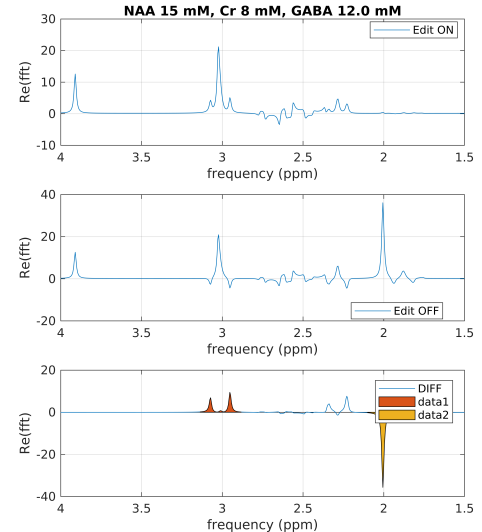

(f) $12.0 \mathrm{mmol}$

Figure D.1: FID-A simulated series with Near et al. GABA model, $15 \mathrm{mmol} \mathrm{NAA}, 8 \mathrm{mmol}$ creatine. 

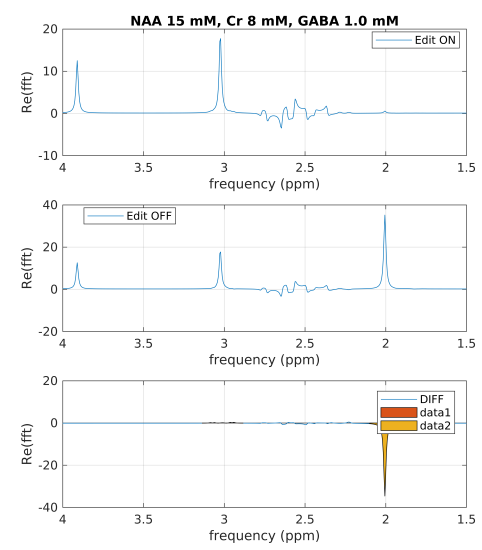

(g) $1.0 \mathrm{mmol}$
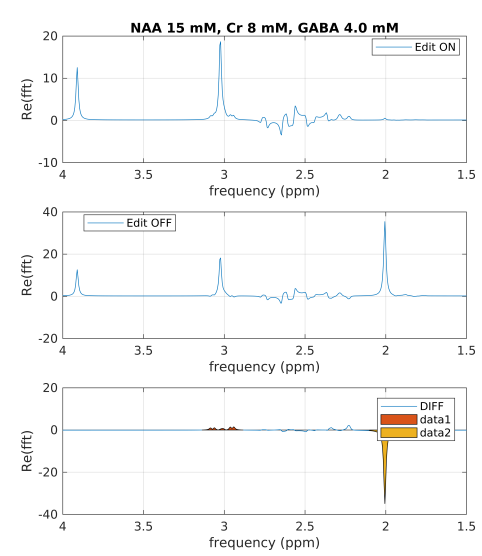

(j) $4.0 \mathrm{mmol}$
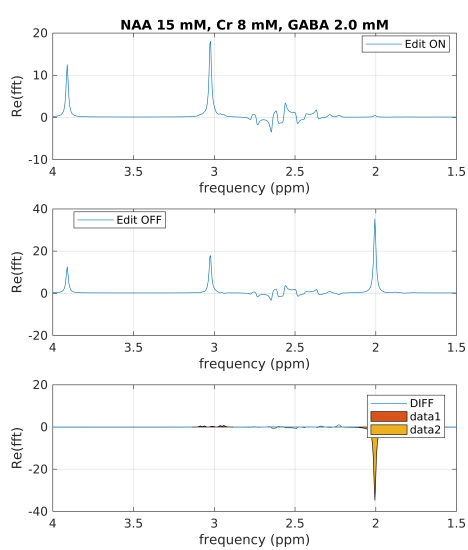

(h) $2.0 \mathrm{mmol}$
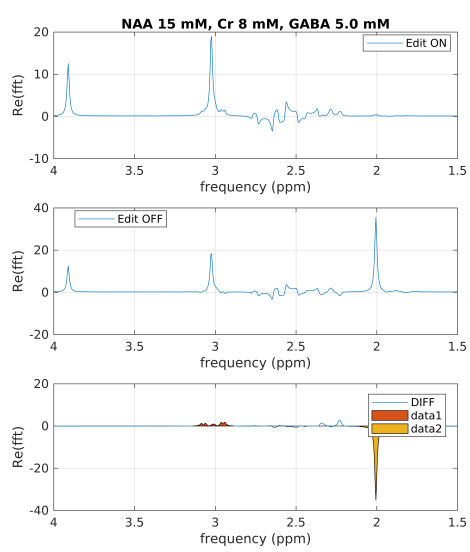

(k) $5.0 \mathrm{mmol}$

Figure D.2
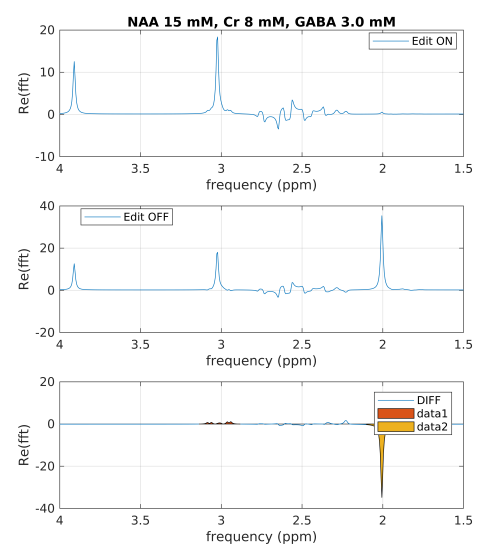

(i) $3.0 \mathrm{mmol}$
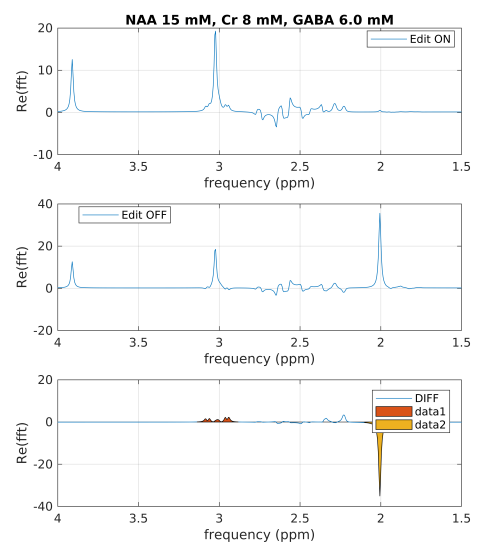

(l) $6.0 \mathrm{mmol}$ 

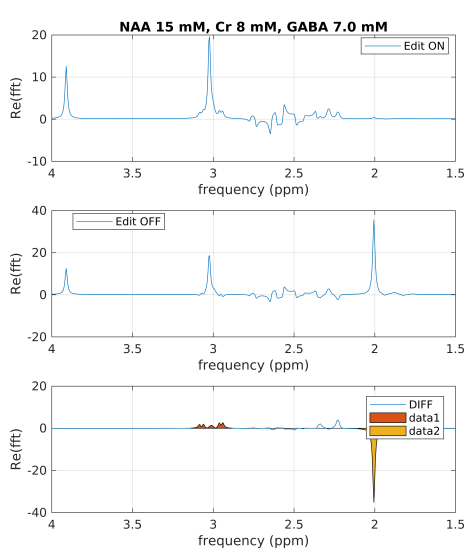

(a) $7.0 \mathrm{mmol}$
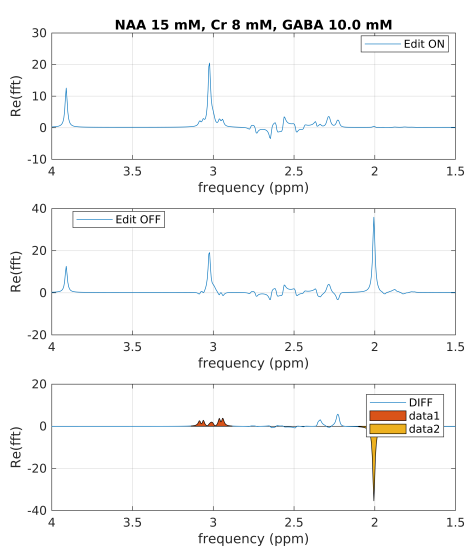

(d) $10.0 \mathrm{mmol}$
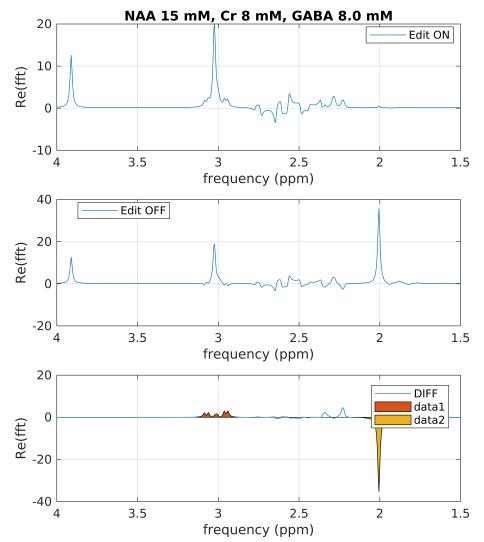

(b) $8.0 \mathrm{mmol}$
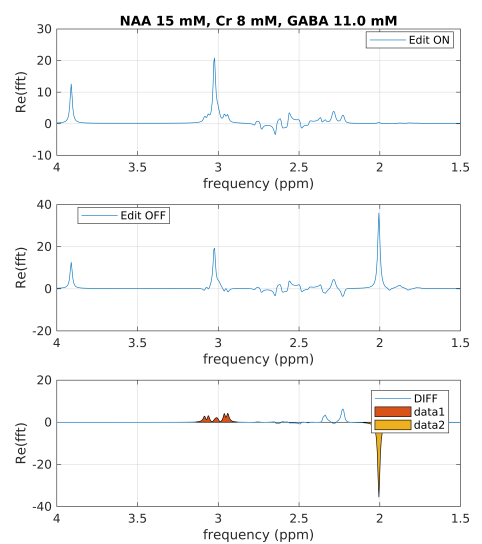

(e) $11.0 \mathrm{mmol}$
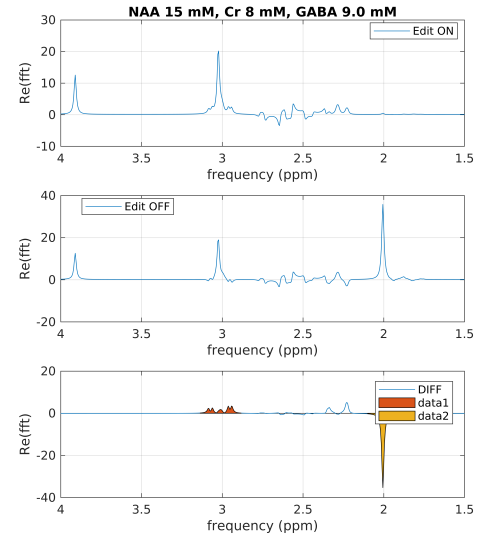

(c) $9.0 \mathrm{mmol}$
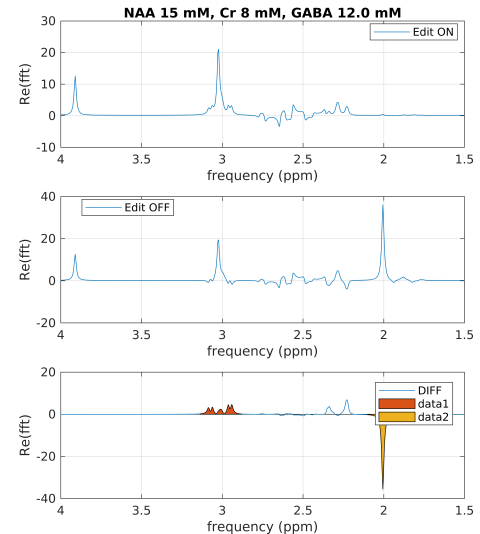

(f) $12.0 \mathrm{mmol}$

Figure D.2: FID-A simulated series with Govindaraju et al. GABA model, 15 mmol NAA, 8 mmol creatine. 

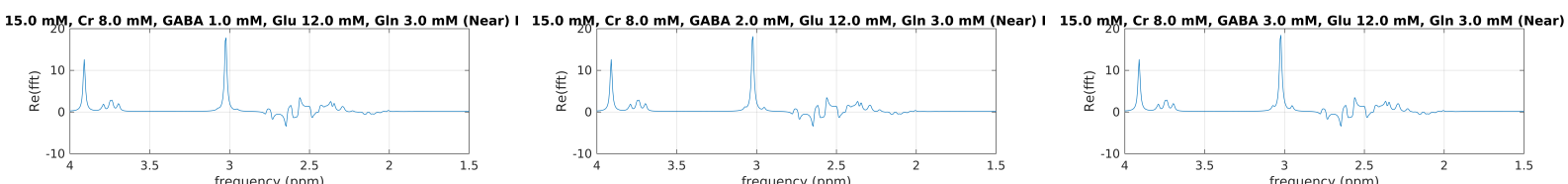

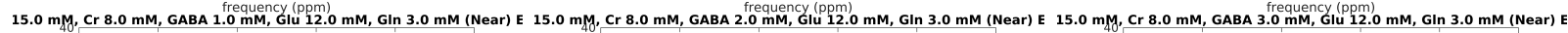
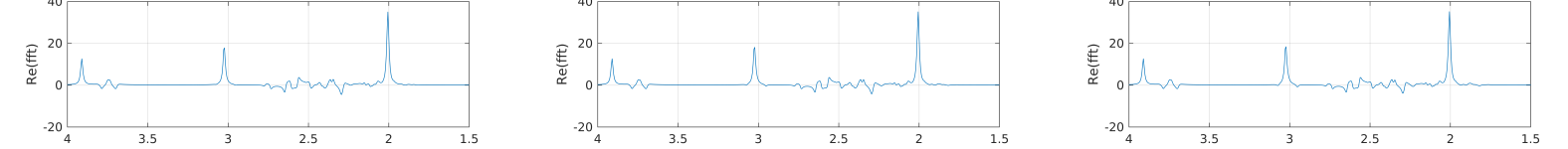

$15.0 \mathrm{mM}, \mathrm{Cr} 8.0 \mathrm{mM}$, GABA $1.0 \mathrm{mM}$, Glu $12.0 \mathrm{mM}$, Gln $3.0 \mathrm{mM}$ (
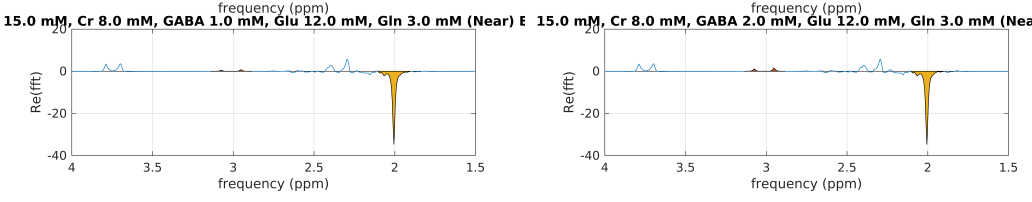

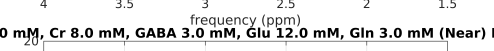

(g) $1.0 \mathrm{mmol}$

(h) $2.0 \mathrm{mmol}$

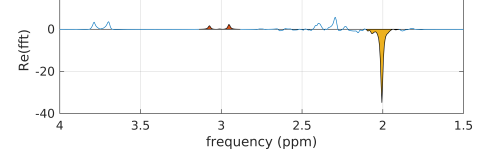

(i) $3.0 \mathrm{mmol}$
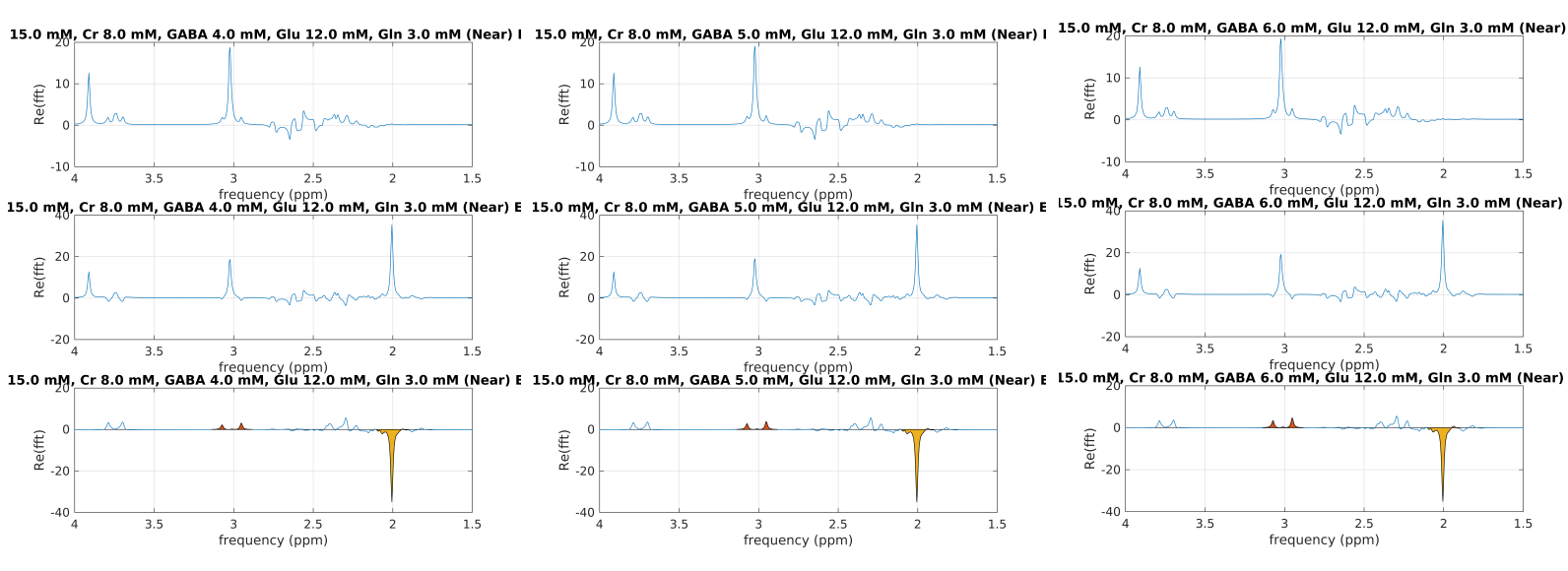

(j) $4.0 \mathrm{mmol}$
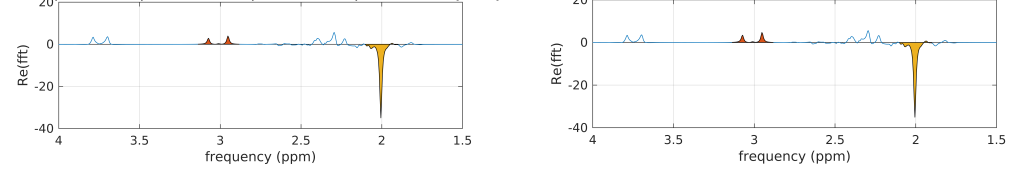

(k) $5.0 \mathrm{mmol}$

(l) $6.0 \mathrm{mmol}$

Figure D.3 

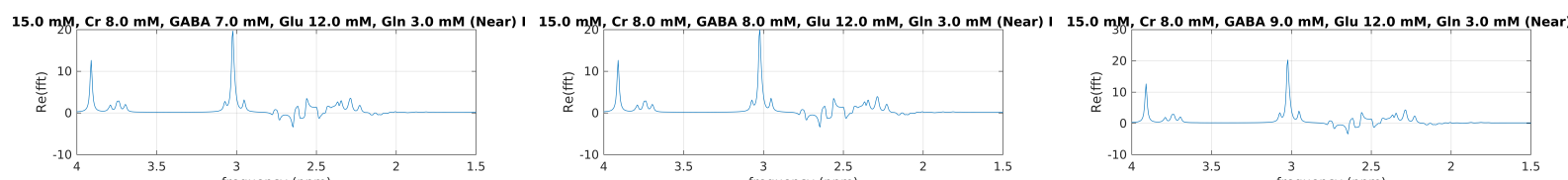

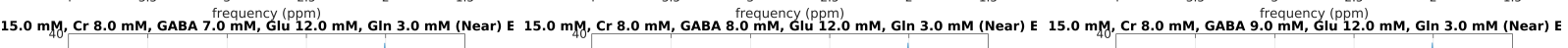
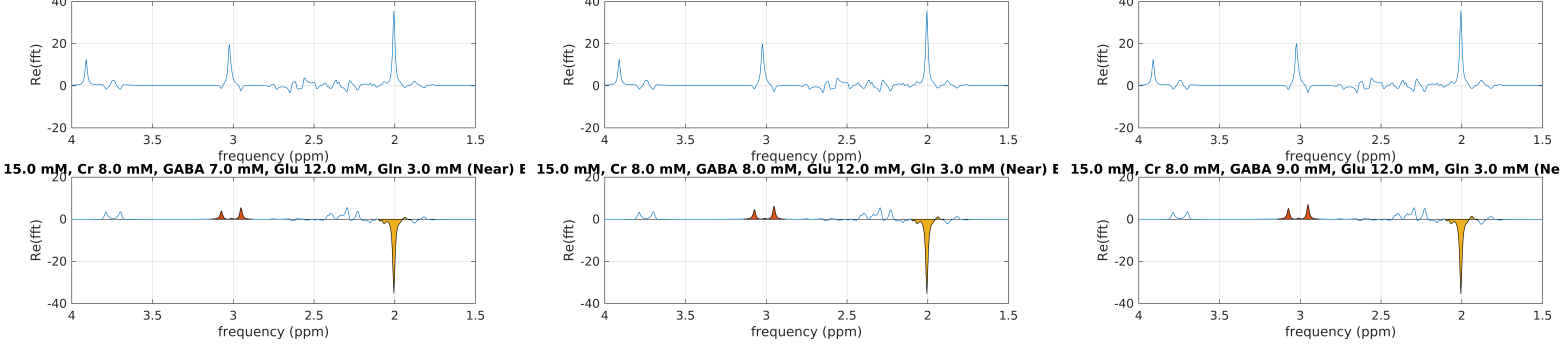

(a) $7.0 \mathrm{mmol}$

(b) $8.0 \mathrm{mmol}$

(c) $9.0 \mathrm{mmol}$

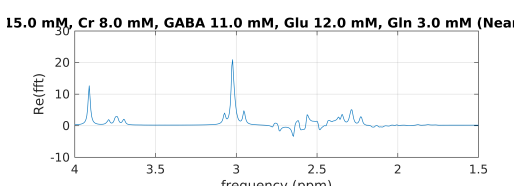

$.5 .0 \mathrm{mM}, \mathrm{Cr} 8.0 \mathrm{mM}, \mathrm{GABA} 11.0 \mathrm{mM}, \mathrm{Grequ} 12.0 \mathrm{mM}, \mathrm{Gln} 3.0 \mathrm{mM}$ (Near)
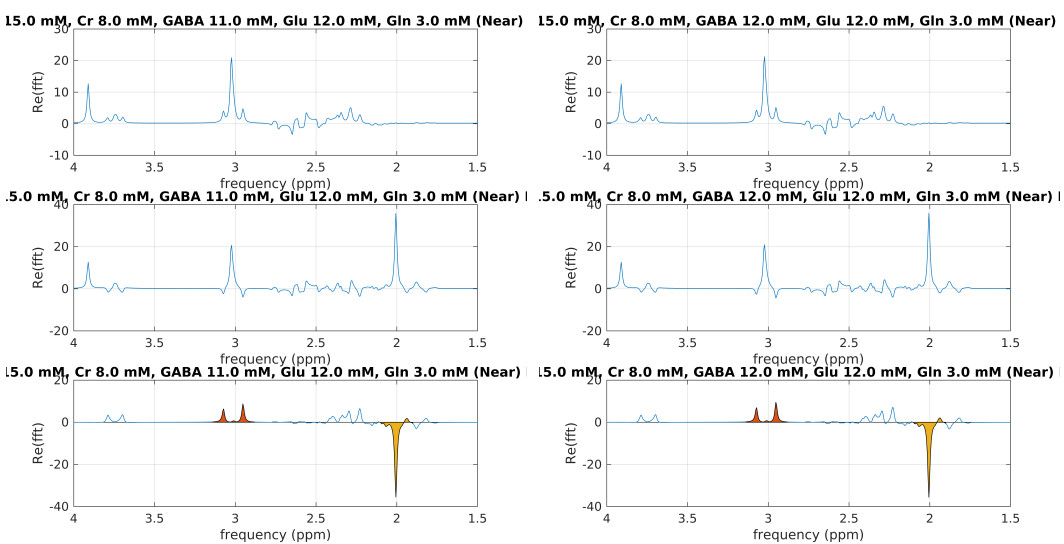

(d) $11.0 \mathrm{mmol}$

(e) $12.0 \mathrm{mmol}$

Figure D.3: FID-A simulated series with Near et al. GABA model, 15 mmol NAA, 8 mmol creatine, $12 \mathrm{mmol}$ glutamate, $3 \mathrm{mmol}$ glutamine. 

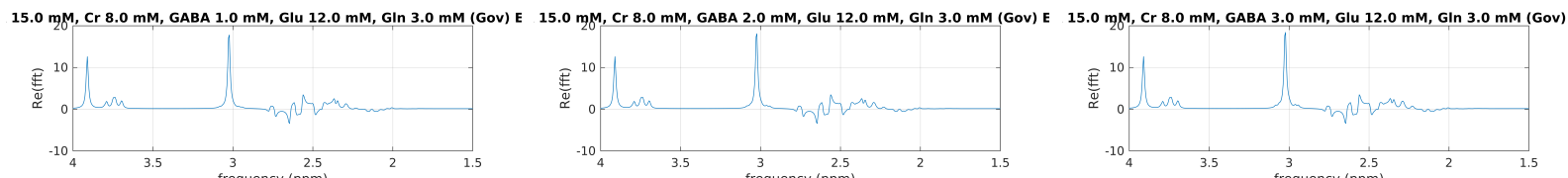

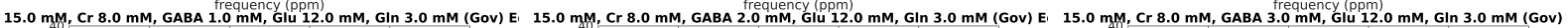
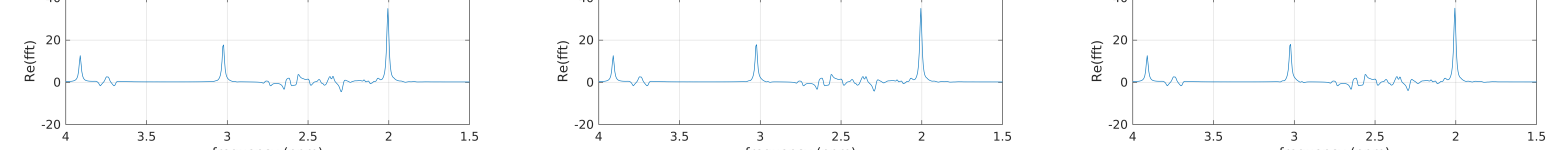

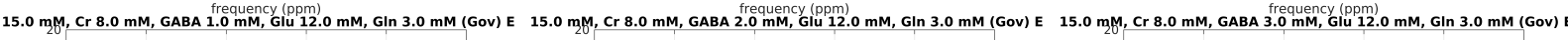
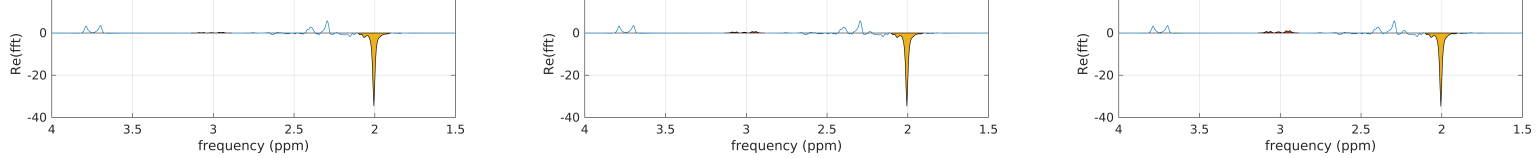

(f) $1.0 \mathrm{mmol}$

(g) $2.0 \mathrm{mmol}$

(h) $3.0 \mathrm{mmol}$

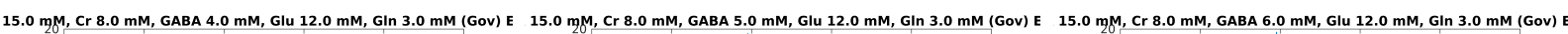
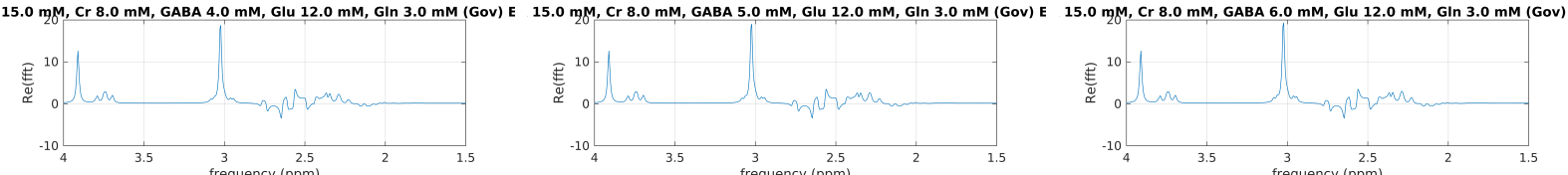

$15.0 \mathrm{mM}, \mathrm{Cr} 8.0 \mathrm{mM}$, GABA $4.0 \mathrm{mMM}$, Glu $12.0 \mathrm{mM}, \mathrm{Gln} 3.0 \mathrm{mM}$ (Gov) E1 $15.0 \mathrm{mM}, \mathrm{Cr} 8.0 \mathrm{mM}, \mathrm{GABA} 5.0 \mathrm{mM}, \mathrm{Glu} 12.0 \mathrm{mM}, \mathrm{Gln} 3.0 \mathrm{mM}$ (Gov) E

mM, Cr $8.0 \mathrm{mM}$, GABA frequency 6 mM, Glu $12.0 \mathrm{mM}, \mathrm{Gln} 3.0 \mathrm{mM}$ (Gov) E:
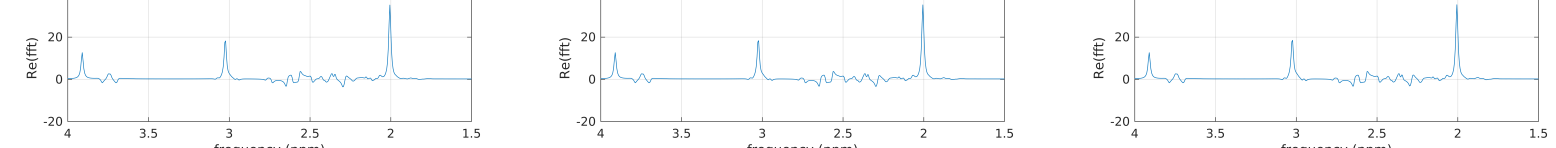

$15.0 \mathrm{mM}, \mathrm{Cr} 8.0 \mathrm{mM}$, GABA $4.0 \mathrm{mM}$, Glu $12.0 \mathrm{mM}, \mathrm{Gln} 3.0 \mathrm{mM}$ (Gov) E $15.0 \mathrm{mM}, \mathrm{Cr} 8.0 \mathrm{mM}$, GABA $5.0 \mathrm{mM}$, Glu $12.0 \mathrm{mM}, \mathrm{Gln} 3.0 \mathrm{mM}$ (Gov)
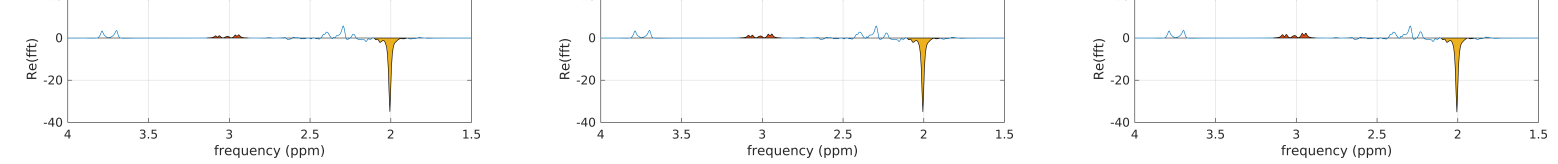

(i) $4.0 \mathrm{mmol}$

(j) $5.0 \mathrm{mmol}$

(k) $6.0 \mathrm{mmol}$

Figure D.4 

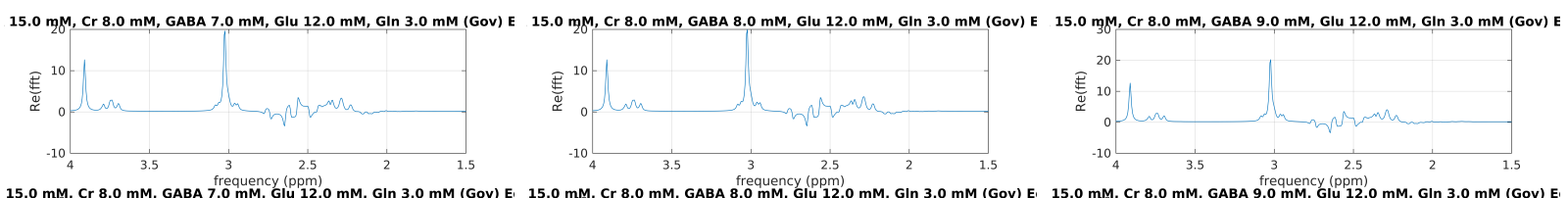

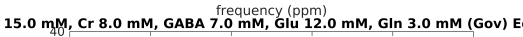
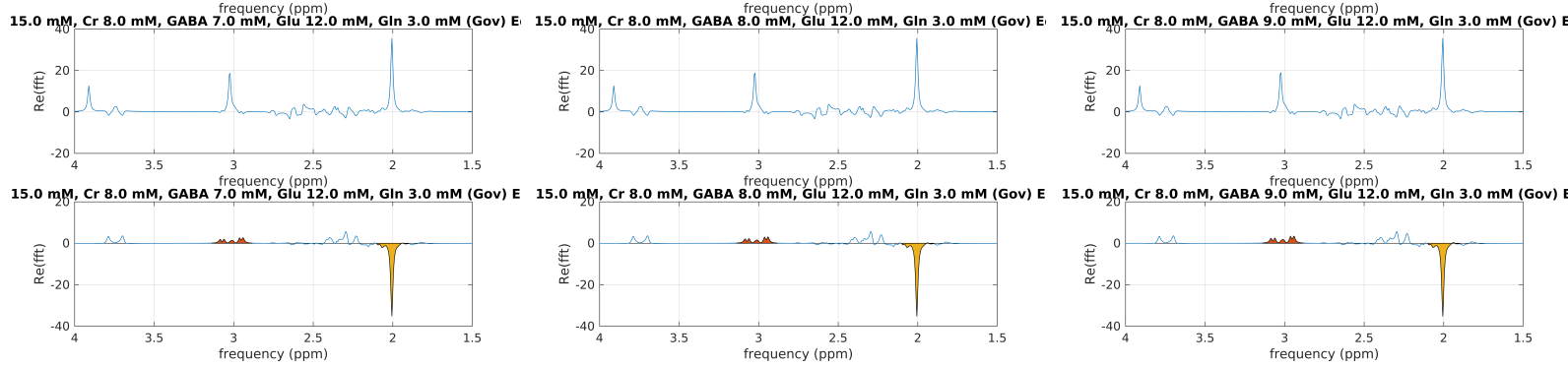
(a) $7.0 \mathrm{mmol}$
(b) $8.0 \mathrm{mmol}$
(c) $9.0 \mathrm{mmol}$
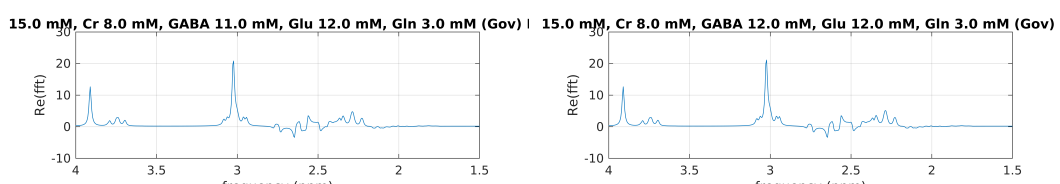

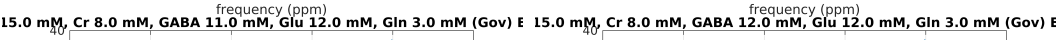
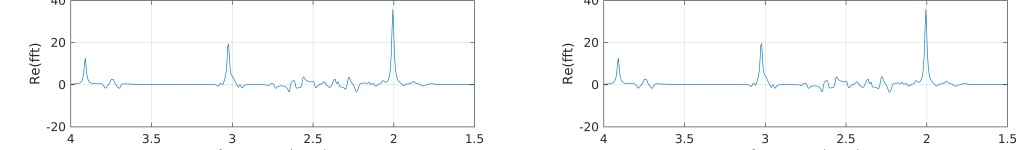

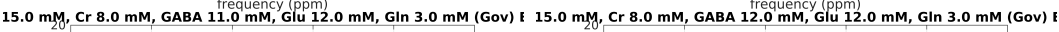
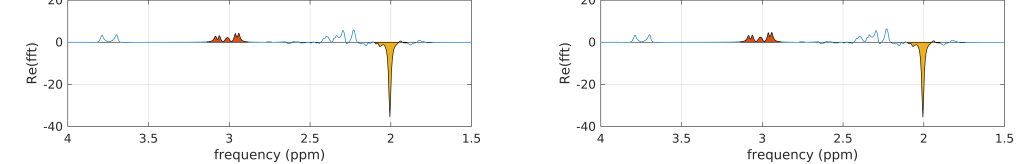

(d) $11.0 \mathrm{mmol}$

(e) $12.0 \mathrm{mmol}$

Figure D.4: FID-A simulated series with Govindaraju et al. GABA model, 15 mmol NAA, $8 \mathrm{mmol}$ creatine, $12 \mathrm{mmol}$ glutamate, $3 \mathrm{mmol}$ glutamine. 

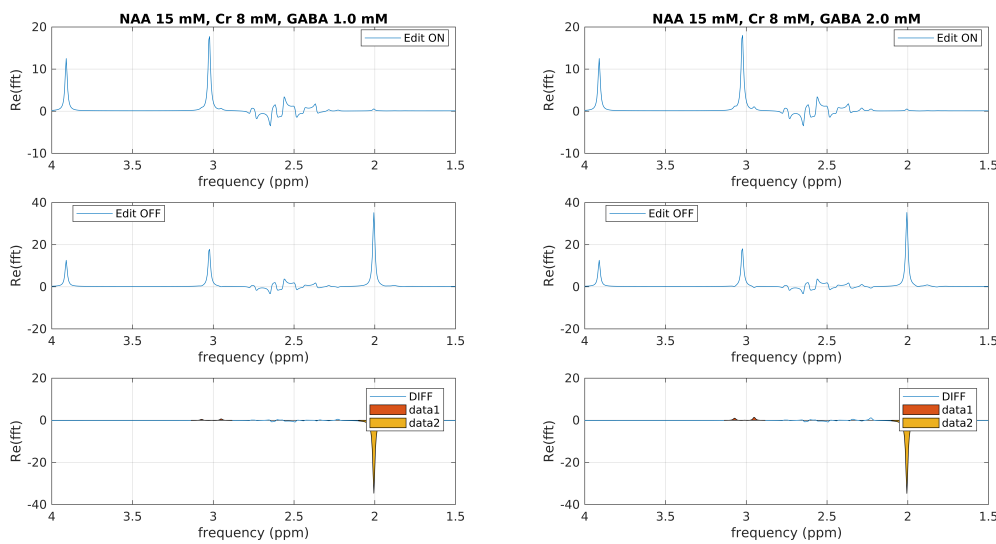

(f) $1.0 \mathrm{mmol}$
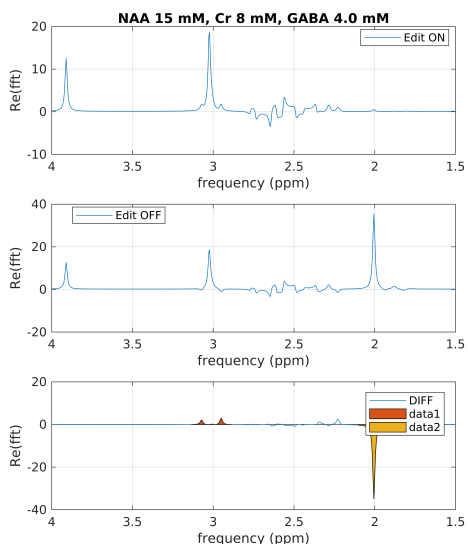

(i) $4.0 \mathrm{mmol}$
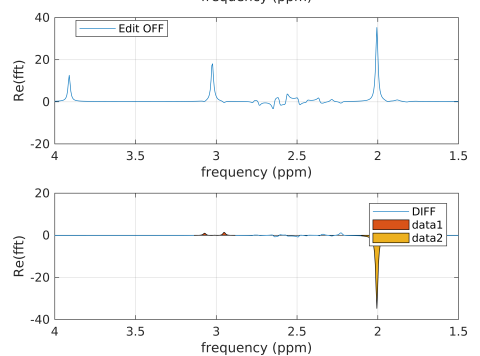

(g) $2.0 \mathrm{mmol}$
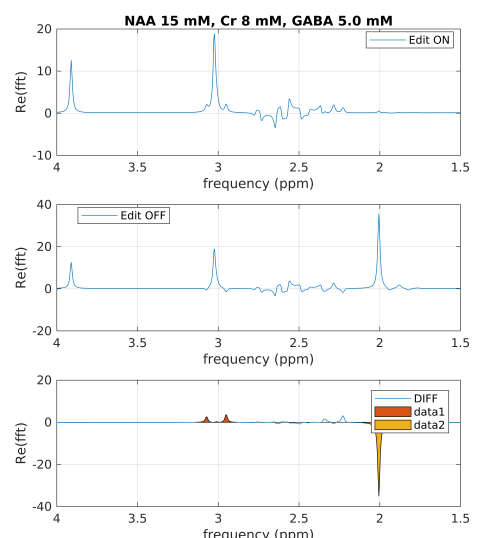

(j) $5.0 \mathrm{mmol}$
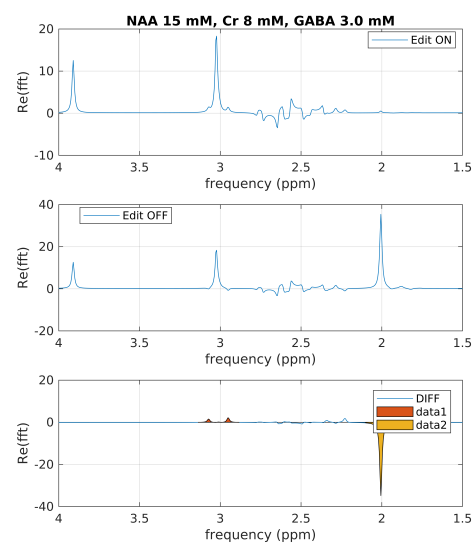

(h) $3.0 \mathrm{mmol}$
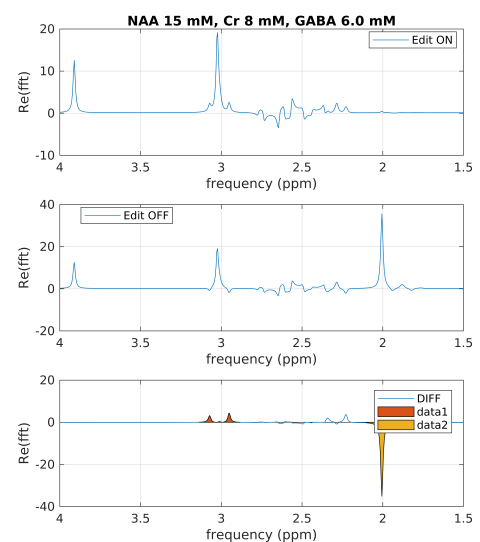

(k) $6.0 \mathrm{mmol}$

Figure D.5 

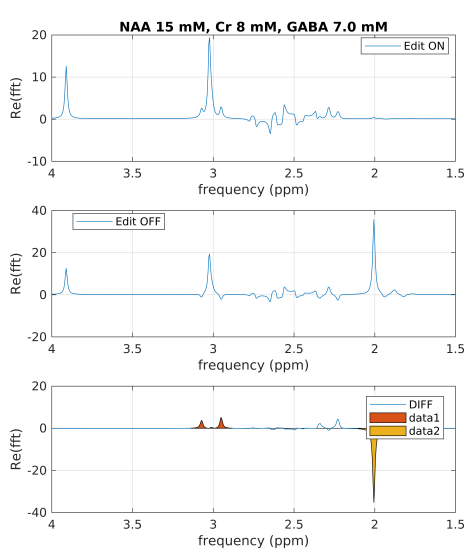

(a) $7.0 \mathrm{mmol}$
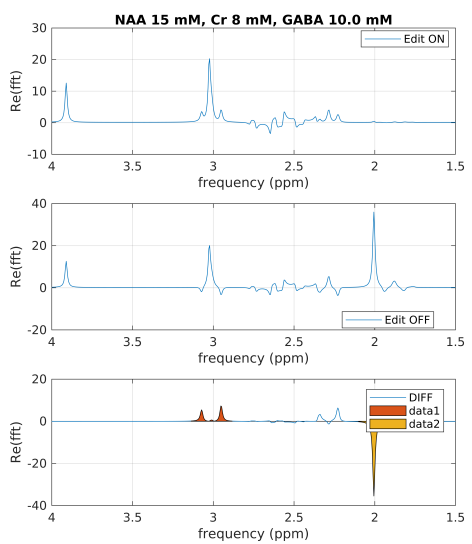

(d) $10.0 \mathrm{mmol}$
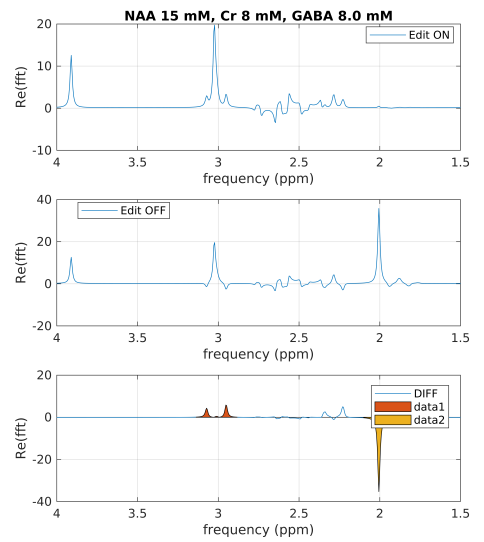

(b) $8.0 \mathrm{mmol}$
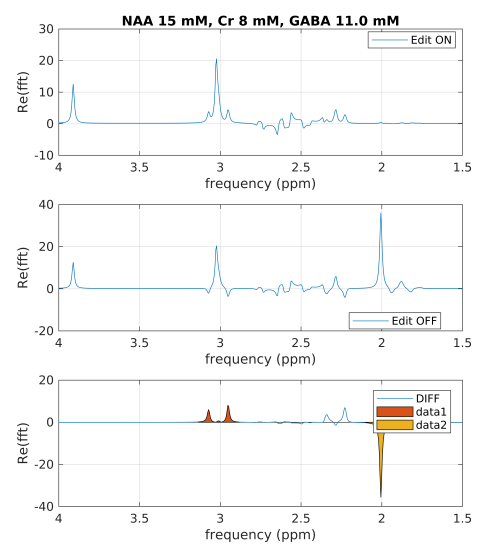

(e) $11.0 \mathrm{mmol}$
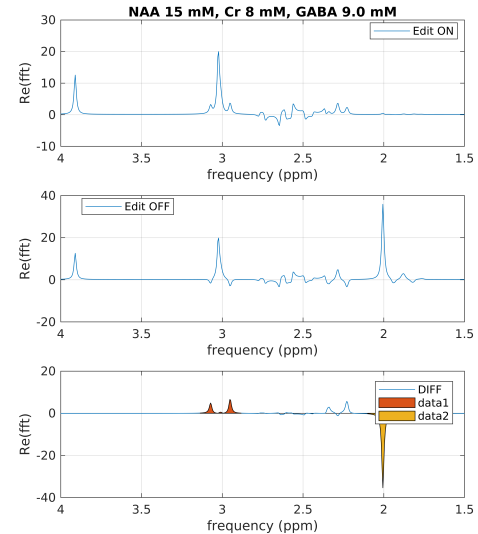

(c) $9.0 \mathrm{mmol}$
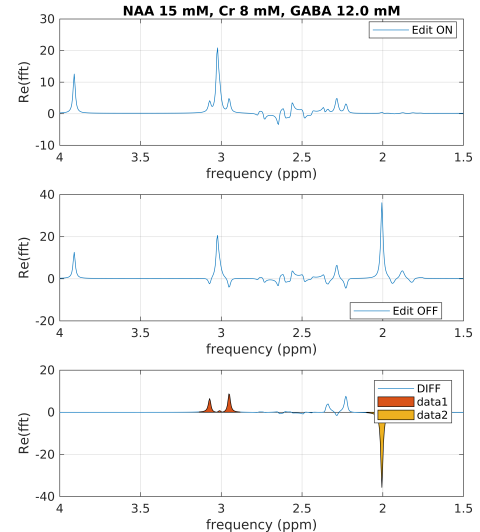

(f) $12.0 \mathrm{mmol}$

Figure D.5: FID-A simulated series with Kaiser et al. GABA model, 15 mmol NAA, $8 \mathrm{mmol}$ creatine, $12 \mathrm{mmol}$ glutamate, $3 \mathrm{mmol}$ glutamine. 


\section{References}

[1] V. Govindaraju, K. Young, and A. A. Maudsley, "Proton NMR chemical shifts and coupling constants for brain metabolites," NMR in Biomedicine, vol. 13, no. 3, pp. 129 $153,2000$.

[2] L. G. Kaiser, K. Young, D. J. Meyerhoff, S. G. Mueller, and G. B. Matson, "A detailed analysis of localized J-difference GABA editing: theoretical and experimental study at 4T," NMR in Biomedicine, vol. 21, pp. 22-32, jan 2008.

[3] J. Near, E. John, N. A. Puts, P. B. Barker, and R. Edden, "J-difference editing of GABA: simulated and experimental multiplet patterns," Magnetic Resonance in Medicine, vol. 193, no. 1, pp. 118-125, 2011.

[4] P. C. Lauterbur, "Image formation by induced local interactions: Examples employing nuclear magnetic resonance," Nature, vol. 242, pp. 190-191, mar 1973.

[5] P. Mansfield and A. A. Maudsley, "Planar spin imaging by NMR," Journal of Magnetic Resonance (1969), vol. 27, no. 1, pp. 101-119, 1977.

[6] P. Mansfield and A. A. Maudsley, "Medical imaging by NMR," British Journal of Radiology, vol. 50, no. 591, pp. 188-194, 1977.

[7] OECD, "Number of magnetic resonance imaging (MRI) units and computed tomography (CT) scanners.," Health, vol. 120, pp. 363-364, 2010.

[8] National Health Service, "NHS Imaging and Radiodiagnostic activity in England," no. August, pp. 1-7, 2013.

[9] S. Dixon, "Classification: Official 1 Diagnostic Imaging Dataset Statistical Release Classification: Official 2 Diagnostic Imaging Dataset Statistical Release,” 2017.

[10] J. Z. Bojorquez, S. Bricq, C. Acquitter, F. Brunotte, P. M. Walker, and A. Lalande, "What are normal relaxation times of tissues at 3 T?," 2017. 
[11] F. S. U. Bloch, W. W. S. U. Hansen, and M. S. U. Packard, "Nuclear Induction," Physical Review, vol. 70, no. 69, pp. 474-485, 1946.

[12] R. A. Graaf, In vivo NMR spectroscopy - Principles and techniques. Wiley, 2007.

[13] F. Bloch, A. Siegert, I. I. Rabi, S. Millman, P. Kusch, L. W. Alvarez, and P. Rev, "Magnetic Resonance for Nonrotating Fields," Phys. Rev, vol. 55, no. 526, 1939.

[14] D. Wishart, C. Bigam, J. Yao, F. Abildgaard, H. Dyson, E. Oldfield, J. Markley, and B. Sykes, "1H, 13C and 15N chemical shift referencing in biomolecular NMR," Journal of Biomolecular NMR, vol. 6, pp. 135-140, sep 1995.

[15] M. Martin, J. Labouesse, P. Canioni, and M. Merle, "N-Acetyl-L-Aspartate and Acetate ' H NMR Signal Overlapping under Mild Acidic pH Conditions," Magnetic Resonance in Medicine, vol. 29, no. 5, pp. 692-694, 2005.

[16] B. Karaszewski, J. M. Wardlaw, I. Marshall, V. Cvoro, K. Wartolowska, K. Haga, P. A. Armitage, M. E. Bastin, and M. S. Dennis, "Measurement of brain temperature with magnetic resonance spectroscopy in acute ischemic stroke," Annals of Neurology, vol. 60, no. 4, pp. 438-446, 2006.

[17] I. Tkáč, Z. Starčuk, I.-Y. Choi, and R. Gruetter, "In Vivo 1 H NMR Spectroscopy of Rat Brain at $1 \mathrm{~ms}$ Echo Time," Magnetic Resonance inMedicine, vol. 41, no. 4, pp. 649-656, 1999.

[18] C. T. W. Moonen, M. V. Kienlin, P. C. M. Van Zijl, J. Cohen, J. Gillen, P. Daly, and G. Wolf, "Comparison of single-shot localization methods (steam and press) forIn vivo proton NMR spectroscopy," NMR in Biomedicine, vol. 2, pp. 201-208, dec 1989.

[19] D. G. Taylor, R. Inamdar, M. C. Bushell, A. Haase, J. Frahm, W. Hanicke, and D. Matthaei, "Related content Imaging by nuclear magnetic resonance P MansfieldNMR imaging in theory and in practice 'H NMR chemical shift selective (CHESS) imaging?," Tech. Rep. 4, 1985. 
[20] J. Star-Lack, S. J. Nelson, J. Kurhanewicz, L. R. Huang, and D. B. Vigneron, "Improved water and lipid suppression for 3D PRESS CSI using RF band selective inversion with gradient dephasing (BASING)," Magnetic Resonance in Medicine, vol. 38, pp. 311-321, aug 1997.

[21] I. Tkáč, Z. Starčuk, I. Y. Choi, and R. Gruetter, "In vivo1H NMR spectroscopy of rat brain at 1 ms echo time," Magnetic Resonance in Medicine, vol. 41, pp. 649-656, apr 1999.

[22] R. J. Ogg, P. B. Kingsley, and J. S. Taylor, "WET, a T1- and B1-Insensitive WaterSuppression Method for in Vivo Localized 1H NMR Spectroscopy," Journal of Magnetic Resonance, Series B, vol. 104, pp. 1-10, may 1994.

[23] M. Mescher, R. Gruetter, H. Merkle, and M. Garwood, "Water suppression using selective echo dephasing," in Proceedings of the ISMRM 4th Scientific Meeting and Exhibition, vol. 123, p. 384, 1996.

[24] M. Mescher, H. Merkle, J. Kirsch, M. Garwood, and R. Gruetter, "Simultaneous in vivo spectral editing and water suppression," NMR in Biomedicine, vol. 11, no. 6, pp. 266-272, 1998.

[25] P. G. Mullins, D. J. McGonigle, R. L. O'Gorman, N. A. J. Puts, R. Vidyasagar, C. J. Evans, R. Edden, M. J. Brookes, A. Garcia, B. R. Foerster, M. Petrou, D. Price, B. S. Solanky, I. R. Violante, S. Williams, and M. Wilson, "Current practice in the use of MEGA-PRESS spectroscopy for the detection of GABA," NeuroImage, vol. 86, pp. $43-52,2014$.

[26] N. A. J. Puts and R. Edden, "In vivo magnetic resonance spectroscopy of GABA: A methodological review," Progress in Nuclear Magnetic Resonance Spectroscopy, vol. 60, pp. 29-41, 2012.

[27] K. W. Waddell, M. J. Avison, J. M. Joers, and J. C. Gore, "A practical guide to robust detection of GABA in human brain by J-difference spectroscopy at $3 \mathrm{~T}$ using 
a standard volume coil," Magnetic Resonance Imaging, vol. 25, no. 7, pp. 1032-1038, 2007.

[28] M. Garwood and L. DelaBarre, "The return of the frequency sweep: Designing adiabatic pulses for contemporary NMR," Journal of Magnetic Resonance, vol. 153, no. 2, pp. 155-177, 2001.

[29] Y. Ke, B. M. Cohen, J. Y. Bang, M. Yang, and P. F. Renshaw, "PSYCHIATRY RESEARCH NEUROIMAGING Assessment of GABA concentration in human brain using two-dimensional proton magnetic resonance spectroscopy," www.el sevierxomilocateipsychresns Psychiatry Research: Neuroimaging Section, vol. 1000, pp. 169-178, $\operatorname{dec} 2000$.

[30] R. B. Lydiard, "The role of GABA in anxiety disorders," 2003.

[31] C. Preibisch and R. Deichmann, "Influence of RF spoiling on the stability and accuracy of T1 mapping based on spoiled FLASH with varying flip angles," Magnetic Resonance in Medicine, vol. 61, no. 1, pp. 125-135, 2009.

[32] O. Bane, S. J. Hectors, M. Wagner, L. L. Arlinghaus, M. P. Aryal, Y. Cao, T. L. Chenevert, F. Fennessy, W. Huang, N. M. Hylton, J. Kalpathy-Cramer, K. E. Keenan, D. I. Malyarenko, R. V. Mulkern, D. C. Newitt, S. E. Russek, K. F. Stupic, A. Tudorica, L. J. Wilmes, T. E. Yankeelov, Y. F. Yen, M. A. Boss, and B. Taouli, "Accuracy, repeatability, and interplatform reproducibility of T1quantification methods used for DCE-MRI: Results from a multicenter phantom study," Magnetic Resonance in Medicine, vol. 79, no. 5, pp. 2564-2575, 2018.

[33] A. Haase, J. Frahm, D. Matthaei, W. Hanicke, and K. D. Merboldt, "FLASH imaging. Rapid NMR imaging using low flip-angle pulses," Journal of Magnetic Resonance (1969), vol. 67, no. 2, pp. 258-266, 1986.

[34] P. J. Wright, O. E. Mougin, J. J. Totman, A. M. Peters, M. J. Brookes, R. Coxon, P. E. Morris, M. Clemence, S. T. Francis, R. W. Bowtell, and P. A. Gowland, "Water proton T1measurements in brain tissue at 7,3 , and $1.5 \mathrm{~T}$ using IR-EPI, IR-TSE, 
and MPRAGE: Results and optimization," Magnetic Resonance Materials in Physics, Biology and Medicine, vol. 21, no. 1-2, pp. 121-130, 2008.

[35] C. Preibisch and R. Deichmann, "T1 mapping using spoiled FLASH-EPI hybrid sequences and varying flip angles," Magnetic Resonance in Medicine, vol. 62, no. 1, pp. 240-246, 2009.

[36] A. B. Boizán, G. Gambarota, F. Noury, and H. Saint-Jalmes, "A single phantom to mimic $<\sup >1</$ sup $>$ H MR spectra of different tissues," Concepts in Magnetic Resonance Part A: Bridging Education and Research, vol. 43, no. 4, pp. 138-145, 2015.

[37] M. G. Saleh, G. Oeltzschner, K. L. Chan, N. A. J. Puts, M. Mikkelsen, M. Schär, A. D. Harris, and R. Edden, "Simultaneous edited MRS of GABA and glutathione," NeuroImage, vol. 142, pp. 576-582, 2016.

[38] R. A. Edden, G. Oeltzschner, A. D. Harris, N. A. Puts, K. L. Chan, V. O. Boer, M. Schär, and P. B. Barker, "Prospective frequency correction for macromoleculesuppressed GABA editing at 3T," Journal of Magnetic Resonance Imaging, vol. 44, no. 6, pp. 1474-1482, 2016.

[39] T. M. van Veenendaal, W. H. Backes, F. C. van Bussel, R. A. Edden, N. A. Puts, A. P. Aldenkamp, and J. F. Jansen, "Glutamate quantification by PRESS or MEGAPRESS: Validation, repeatability, and concordance," Magnetic Resonance Imaging, vol. 48, no. September 2017, pp. 107-114, 2018.

[40] N. Fatin-Rouge, K. Starchev, and J. Buffle, "Size effects on diffusion processes within agarose gels," Biophysical Journal, vol. 86, no. 5, pp. 2710-2719, 2004.

[41] I. Lavdas, K. C. Behan, A. Papadaki, D. W. McRobbie, and E. O. Aboagye, "A phantom for diffusion-weighted MRI (DW-MRI)," Journal of Magnetic Resonance Imaging, vol. 38, no. 1, pp. 173-179, 2013. 
[42] Z. G. Portakal, S. Shermer, C. Jenkins, E. Spezi, T. Perrett, N. Tuncel, and J. Phillips, "Design and characterization of tissue-mimicking gel phantoms for diffusion kurtosis imaging," Medical Physics, vol. 45, pp. 2476-2485, jun 2018.

[43] G. Lalwani, J. L. Sundararaj, K. Schaefer, T. Button, and B. Sitharaman, "Synthesis, characterization, in vitro phantom imaging, and cytotoxicity of a novel graphene-based multimodal magnetic resonance imaging-X-ray computed tomography contrast agent," Journal of Materials Chemistry B, vol. 2, no. 22, pp. 3519-3530, 2014.

[44] W. D. D’Souza, E. L. Madsen, O. Unal, K. K. Vigen, G. R. Frank, and B. R. Thomadsen, "Tissue mimicking materials for a multi-imaging modality prostate phantom," Medical Physics, vol. 28, no. 4, pp. 688-700, 2001.

[45] A. Hellerbach, V. Schuster, A. Jansen, and J. Sommer, "MRI Phantoms - Are There Alternatives to Agar?," PLoS ONE, vol. 8, no. 8, 2013.

[46] J. O. Christoffersson, L. E. Olsson, and S. Sjöberg, "Nickel-doped agarose gel phantoms in MR imaging," Acta Radiologica, vol. 32, pp. 426-431, sep 1991.

[47] F. A. Howe, "Relaxation times in paramagnetically doped agarose gels as a function of temperature and ion concentration," Magnetic Resonance Imaging, vol. 6, pp. 263-270, may 1988.

[48] K. A. Kraft, P. P. Fatouros, G. D. Clarke, and P. R. S. Kishore, "An MRI phantom material for quantitative relaxometry," Magnetic Resonance in Medicine, vol. 5, pp. 555-562, dec 1987.

[49] P. Walker, R. Lerski, R. Mathur- De Vre, J. Binet, and F. Yane, "Preparation of agarose gels as reference substances for NMR relaxation time measurement," Magnetic Resonance Imaging, vol. 6, pp. 215-222, 1988.

[50] J. C. Blechinger, E. L. Madsen, and G. R. Frank, "Tissue mimicking gelatin-agar gels for use in magnetic resonance imaging phantoms," jul 1988. 
[51] V. I. Lozinsky, I. Y. Galaev, F. M. Plieva, I. N. Savina, H. Jungvid, and B. Mattiasson, "Polymeric cryogels as promising materials of biotechnological interest," Trends in Biotechnology, vol. 21, no. 10, pp. 445-451, 2003.

[52] N. E. Vrana, Use of Poly Vinyl Alcohol ( PVA ) Cryogelation for Tissue Engineering : Composites, Scaffold Formation and Cell Encapsulation. PhD thesis, Dublin City University, 2009.

[53] K. Yoshimura, H. Kato, M. Kuroda, A. Yoshida, K. Hanamoto, A. Tanaka, M. Tsunoda, S. Kanazawa, K. Shibuya, S. Kawasaki, and Y. Hiraki, "Development of a TissueEquivalent MRI Phantom Using Carrageenan Gel," Magnetic Resonance in Medicine, vol. 50, no. 5, pp. 1011-1017, 2003.

[54] A. Bono, S. M. Anisuzzaman, and O. W. Ding, "Effect of process conditions on the gel viscosity and gel strength of semi-refined carrageenan (SRC) produced from seaweed (Kappaphycus alvarezii)," Journal of King Saud University - Engineering Sciences, vol. 26, no. 1, pp. 3-9, 2014.

[55] K. Thangavel and E. Ü. Saritaş, "Aqueous paramagnetic solutions for MRI phantoms at 3 T: A detailed study on relaxivities," Turkish Journal of Electrical Engineering and Computer Sciences, vol. 25, no. 3, pp. 2108-2121, 2017.

[56] A. D. Elster, "An Index System for Comparative Parameter Weighting in MR Imaging," Journal of Computer Assisted Tomography, pp. 130-134, 1988.

[57] G. M. Bydder and I. R. Young, "Mr imaging: Clinical use of the inversion recovery sequence," Journal of Computer Assisted Tomography, pp. 659-675, 1985.

[58] T. Yokoo, W. C. Bae, G. Hamilton, A. Karimi, J. P. Borgstede, B. C. Bowen, C. B. Sirlin, C. B. Chung, J. V. Crues, W. G. Bradley, and G. M. Bydder, "A quantitative approach to sequence and image weighting," Journal of Computer Assisted Tomography, vol. 34, no. 3, pp. 317-331, 2010. 
[59] S. C. Deoni, B. K. Rutt, A. G. Parrent, and T. M. Peters, "Segmentation of thalamic nuclei using a modified k-means clustering algorithm and high-resolution quantitative magnetic resonance imaging at 1.5 T," NeuroImage, vol. 34, no. 1, pp. 117-126, 2007.

[60] D. Ma, V. Gulani, N. Seiberlich, K. Liu, J. L. Sunshine, J. L. Duerk, and M. A. Griswold, "Magnetic resonance fingerprinting," Nature, vol. 495, no. 7440, pp. 187192, 2013.

[61] T. D. Karamitsos, S. K. Piechnik, S. M. Banypersad, M. Fontana, N. B. Ntusi, V. M. Ferreira, C. J. Whelan, S. G. Myerson, M. D. Robson, P. N. Hawkins, S. Neubauer, and J. C. Moon, "Noncontrast T1 mapping for the diagnosis of cardiac amyloidosis," JACC: Cardiovascular Imaging, vol. 6, no. 4, pp. 488-497, 2013.

[62] D. R. Messroghli, K. Walters, S. Plein, P. Sparrow, M. G. Friedrich, J. P. Ridgway, and M. U. Sivananthan, "Myocardial T1 mapping: Application to patients with acute and chronic myocardial infarction," Magnetic Resonance in Medicine, vol. 58, no. 1, pp. 34-40, 2007.

[63] M. Ugander, A. J. Oki, L. Y. Hsu, P. Kellman, A. Greiser, A. H. Aletras, C. T. Sibley, M. Y. Chen, W. Patricia Bandettini, and A. E. Arai, "Extracellular volume imaging by magnetic resonance imaging provides insights into overt and sub-clinical myocardial pathology," European Heart Journal, vol. 33, no. 10, pp. 1268-1278, 2012.

[64] C. L. Hoad, N. Palaniyappan, P. Kaye, Y. Chernova, M. W. James, C. Costigan, A. Austin, L. Marciani, P. A. Gowland, I. N. Guha, S. T. Francis, and G. P. Aithal, "A study of $\mathrm{T}<\mathrm{inf}>1</ \mathrm{inf}>$ relaxation time as a measure of liver fibrosis and the influence of confounding histological factors," NMR in Biomedicine, vol. 28, no. 6, pp. 706-714, 2015.

[65] T. Heye, S. R. Yang, M. Bock, S. Brost, K. Weigand, T. Longerich, H. U. Kauczor, and W. Hosch, "MR relaxometry of the liver: Significant elevation of T1 relaxation time in patients with liver cirrhosis," European Radiology, vol. 22, no. 6, pp. 1224-1232, 2012. 
[66] P. S. Tofts, G. Brix, D. L. Buckley, J. L Evelhoch, E. Henderson, M. V. Knopp, H. B. W. Larsson, T.-Y. Lee, N. a. Mayr, G. J. M. Parker, R. E. Port, J. Taylor, and R. M. Weisskoff, "Estimating Kinetic Parameters From Dynamic Contrast-Enhanced T1-Weighted \tMRI of a Diffusable Tracer: Standardized Quantities and Symbols," J Magn Reson Imag, vol. 10, no. July, pp. 223-232, 1999.

[67] F. M. Fennessy, A. Fedorov, S. N. Gupta, E. J. Schmidt, C. M. Tempany, and R. V. Mulkern, "Practical considerations in T1 mapping of prostate for dynamic contrast enhancement pharmacokinetic analyses," Magnetic Resonance Imaging, vol. 30, no. 9, pp. 1224-1233, 2012.

[68] T. Kanda, T. Fukusato, M. Matsuda, K. Toyoda, H. Oba, J. Kotoku, T. Haruyama, K. Kitajima, and S. Furui, "Gadolinium-based Contrast Agent Accumulates in the Brain Even in Subjects without Severe Renal Dysfunction: Evaluation of Autopsy Brain Specimens with Inductively Coupled Plasma Mass Spectroscopy," Radiology, vol. 276, pp. 228-232, jul 2015.

[69] R. J. McDonald, J. S. McDonald, D. F. Kallmes, M. E. Jentoft, D. L. Murray, K. R. Thielen, E. E. Williamson, and L. J. Eckel, "Intracranial Gadolinium Deposition after Contrast-enhanced MR Imaging," Radiology, vol. 275, no. 3, pp. 772-782, 2015.

[70] P. A. Bottomley, T. H. Foster, R. E. Argersinger, and L. M. Pfeifer, "A review of normal tissue hydrogen NMR relaxation times and relaxation mechanisms from 1-100 MHz: Dependence on tissue type, NMR frequency, temperature, species, excision, and age," Medical Physics, vol. 11, pp. 425-448, jul 1984.

[71] T. Ethofer, I. Mader, U. Seeger, G. Helms, M. Erb, W. Grodd, A. Ludolph, and U. Klose, "Comparison of Longitudinal Metabolite Relaxation Times in Different Regions of the Human Brain at 1.5 and 3 Tesla," Magnetic Resonance in Medicine, vol. 50, no. 6, pp. 1296-1301, 2003.

[72] N. Stikov, M. Boudreau, I. R. Levesque, C. L. Tardif, J. K. Barral, and G. B. Pike, "On the accuracy of T1 mapping: Searching for common ground," Magnetic Resonance in Medicine, vol. 73, no. 2, pp. 514-522, 2015. 
[73] S. C. Deoni, S. C. Williams, P. Jezzard, J. Suckling, D. G. Murphy, and D. K. Jones, "Standardized structural magnetic resonance imaging in multicentre studies using quantitative T1and T2imaging at 1.5 T," NeuroImage, vol. 40, no. 2, pp. 662-671, 2008.

[74] D. C. Look and D. R. Locker, "Time saving in measurement of NMR and EPR relaxation times," Review of Scientific Instruments, vol. 41, no. 2, pp. 250-251, 1970.

[75] J. Homer and M. S. Beevers, "Driven-equilibrium single-pulse observation of T1 relaxation. A reevaluation of a rapid "new" method for determining NMR spin-lattice relaxation times," Journal of Magnetic Resonance, vol. 63, pp. 287-297, jun 1985.

[76] Y. Jiang, D. Ma, N. Seiberlich, V. Gulani, and M. A. Griswold, "MR fingerprinting using fast imaging with steady state precession (FISP) with spiral readout," Magnetic Resonance in Medicine, vol. 74, no. 6, pp. 1621-1631, 2015.

[77] F. J. Massey, "The Kolmogorov-Smirnov Test for Goodness of Fit," Journal of the American Statistical Association, vol. 46, pp. 68-78, mar 1951.

[78] L. W. Johnston, "Student's t -Test," Journal of Quality Technology, vol. 2, pp. 243245 , oct 1970 .

[79] N. Sibtain, F. Howe, and D. Saunders, "The clinical value of proton magnetic resonance spectroscopy in adult brain tumours," Clinical Radiology, vol. 62, pp. 109-119, feb 2007.

[80] A. Shukla-Dave, H. Hricak, O. Akin, C. Yu, K. L. Zakian, K. Udo, P. T. Scardino, J. Eastham, and M. W. Kattan, "Preoperative nomograms incorporating magnetic resonance imaging and spectroscopy for prediction of insignificant prostate cancer," BJU International, vol. 109, pp. 1315-1322, may 2012.

[81] A. M. Lahoti, A. P. Dhok, C. R. Rantnaparkhi, J. S. Rawat, N. U. Chandak, and H. S. Tawari, "Role of magnetic resonance imaging, magnetic resonance spectroscopy and transrectal ultrasound in evaluation of prostatic pathologies with focus on prostate cancer," Polish Journal of Radiology, vol. 83, pp. 37-46, 2018. 
[82] P. Stanwell and C. Mountford, "In vivo proton MR spectroscopy of the breast.," $R a-$ diographics : a review publication of the Radiological Society of North America, Inc, vol. 27 Suppl 1, pp. S253-66, oct 2007.

[83] P. A. T. Baltzer and M. Dietzel, "Breast Lesions: Diagnosis by Using Proton MR Spectroscopy at 1.5 and 3.0 T-Systematic Review and Meta-Analysis," Radiology, vol. 267, pp. 735-746, jun 2013.

[84] R. Banerjee, M. Pavlides, E. M. Tunnicliffe, S. K. Piechnik, N. Sarania, R. Philips, J. D. Collier, J. C. Booth, J. E. Schneider, L. M. Wang, D. W. Delaney, K. A. Fleming, M. D. Robson, E. Barnes, and S. Neubauer, "Multiparametric magnetic resonance for the non-invasive diagnosis of liver disease," Journal of Hepatology, vol. 60, pp. 69-77, jan 2014 .

[85] J. L. Wei, J. C. F. Leung, T. C. W. Loong, G. L. H. Wong, D. K. W. Yeung, R. S. M. Chan, H. L. Y. Chan, A. M. L. Chim, J. Woo, W. C. W. Chu, and V. W. S. Wong, "Prevalence and Severity of Nonalcoholic Fatty Liver Disease in Non-Obese Patients: A Population Study Using Proton-Magnetic Resonance Spectroscopy," American Journal of Gastroenterology, vol. 110, pp. 1306-1314, sep 2015.

[86] R. García-Figueiras, S. Baleato-González, A. R. Padhani, L. Oleaga, J. C. Vilanova, A. Luna, and J. C. C. Gómez, "Proton magnetic resonance spectroscopy in oncology: The fingerprints of cancer?," 2016.

[87] L. A. Brandão and M. Castillo, "Adult Brain Tumors: Clinical Applications of Magnetic Resonance Spectroscopy," nov 2016.

[88] D. Croteau, L. Scarpace, D. Hearshen, J. Gutierrez, J. L. Fisher, J. P. Rock, and T. Mikkelsen, "Correlation between magnetic resonance spectroscopy imaging and image-guided biopsies: Semiquantitative and qualitative histopathological analyses of patients with untreated glioma," Neurosurgery, vol. 49, pp. 823-829, oct 2001.

[89] C. Majós, J. Alonso, C. Aguilera, M. Serrallonga, J. Pérez-Martín, J. J. Acebes, C. Arús, and J. Gili, "Proton magnetic resonance spectroscopy (1H MRS) of human 
brain tumours: assessment of differences between tumour types and its applicability in brain tumour categorization," European Radiology, vol. 13, no. 3, pp. 582-591.

[90] D. A. McCormick, "GABA as an inhibitory neurotransmitter in human cerebral cortex," Journal of Neurophysiology, vol. 62, pp. 1018-1027, nov 1989.

[91] M. D. Simpson, P. Slater, J. F. Deakin, M. C. Royston, and W. J. Skan, "Reduced GABA uptake sites in the temporal lobe in schizophrenia," Neuroscience Letters, vol. 107, pp. 211-215, dec 1989.

[92] N. Soltani, H. Qiu, M. Aleksic, Y. Glinka, F. Zhao, R. Liu, Y. Li, N. Zhang, R. Chakrabarti, T. Ng, T. Jin, H. Zhang, W.-Y. Lu, Z.-P. Feng, G. J. Prud'homme, and Q. Wang, "GABA exerts protective and regenerative effects on islet beta cells and reverses diabetes," Proceedings of the National Academy of Sciences, vol. 108, no. 28, pp. 11692-11697, 2011.

[93] M. K. Brix, L. Ersland, K. Hugdahl, R. Grüner, M.-B. Posserud, Å. Hammar, A. R. Craven, R. Noeske, C. J. Evans, H. B. Walker, T. Midtvedt, and M. K. Beyer, “"Brain MR spectroscopy in autism spectrum disorder - the GABA excitatory/inhibitory imbalance theory revisited"," Frontiers in Human Neuroscience, vol. 9, no. June, pp. 1$12,2015$.

[94] F. Boy, C. J. Evans, R. A. Edden, A. D. Lawrence, K. D. Singh, M. Husain, and P. Sumner, "Dorsolateral prefrontal $\gamma$-aminobutyric acid in men predicts individual differences in rash impulsivity," Biological Psychiatry, vol. 70, pp. 866-872, nov 2011.

[95] G. Ende, S. Cackowski, J. Van Eijk, M. Sack, T. Demirakca, N. Kleindienst, M. Bohus, E. Sobanski, A. Krause-Utz, and C. Schmahl, "Impulsivity and Aggression in Female BPD and ADHD Patients: Association with ACC Glutamate and GABA Concentrations," Neuropsychopharmacology, vol. 41, pp. 410-418, jan 2016.

[96] M. S. Cousins, D. C. S. Roberts, and H. de Wit, "GABAB receptor agonists for the treatment of drug addiction: A review of recent findings," Drug and Alcohol Dependence, vol. 65, pp. 209-220, feb 2002. 
[97] C. E. Robertson, E. M. Ratai, and N. Kanwisher, "Reduced GABAergic Action in the Autistic Brain," Current Biology, vol. 26, pp. 80-85, jan 2016.

[98] G. Sanacora, G. F. Mason, D. L. Rothman, K. L. Behar, F. Hyder, O. A. Petroff, R. M. Berman, D. S. Charney, and J. H. Krystal, "Reduced cortical $\gamma$-aminobutyric acid levels in depressed patients determined by proton magnetic resonance spectroscopy," Archives of General Psychiatry, vol. 56, pp. 1043-1047, nov 1999.

[99] M. Mikkelsen, P. B. Barker, P. K. Bhattacharyya, M. K. Brix, P. F. Buur, K. M. Cecil, K. L. Chan, D. Y. Chen, A. R. Craven, K. Cuypers, M. Dacko, N. W. Duncan, U. Dydak, D. A. Edmondson, G. Ende, L. Ersland, F. Gao, I. Greenhouse, A. D. Harris, N. He, S. Heba, N. Hoggard, T. W. Hsu, J. F. Jansen, A. Kangarlu, T. Lange, R. M. Lebel, Y. Li, C. Y. E. Lin, J. K. Liou, J. F. Lirng, F. Liu, R. Ma, C. Maes, M. MorenoOrtega, S. O. Murray, S. Noah, R. Noeske, M. D. Noseworthy, G. Oeltzschner, J. J. Prisciandaro, N. A. J. Puts, T. P. Roberts, M. Sack, N. Sailasuta, M. G. Saleh, M. P. Schallmo, N. Simard, S. P. Swinnen, M. Tegenthoff, P. Truong, G. Wang, I. D. Wilkinson, H. J. Wittsack, H. Xu, F. Yan, C. Zhang, V. Zipunnikov, H. J. Zöllner, and R. Edden, "Big GABA: Edited MR spectroscopy at 24 research sites," NeuroImage, vol. 159, no. July, pp. 32-45, 2017.

[100] A. D. Harris, N. A. J. Puts, S. A. Wijtenburg, L. M. Rowland, M. Mikkelsen, P. B. Barker, C. J. Evans, and R. Edden, "Normalizing data from GABA-edited MEGAPRESS implementations at 3 Tesla," Magnetic Resonance Imaging, vol. 42, pp. 8-15, 2017.

[101] J. F. Myers, D. J. Nutt, and A. R. Lingford-Hughes, " $\gamma$-aminobutyric acid as a metabolite: Interpreting magnetic resonance spectroscopy experiments," Journal of Psychopharmacology, vol. 30, pp. 422-427, may 2016.

[102] A. Ebel, W. Dreher, and D. Leibfritz, "Effects of zero-filling and apodization on spectral integrals in discrete Fourier-transform spectroscopy of noisy data," Journal of Magnetic Resonance, pp. 330-338, 2006. 
[103] W. Pijnappel, A. van den Boogaart, R. De Beer, and D. Van Ormondt, "SVD-based quantification of magnetic resonance signals," Journal of Magnetic Resonance (1969), vol. 97, pp. 122-134, mar 1992.

[104] L. Hofmann, J. Slotboom, C. Boesch, and R. Kreis, "Characterization of the macromolecule baseline in localized1H-MR spectra of human brain," Magnetic Resonance in Medicine, vol. 46, pp. 855-863, nov 2001.

[105] R. Kreis, J. Slotboom, L. Hofmann, and C. Boesch, "Integrated data acquisition and processing to determine metabolite contents, relaxation times, and macromolecule baseline in single examinations of individual subjects," Magnetic Resonance in Medicine, vol. 54, pp. 761-768, oct 2005.

[106] G. Helms and A. Piringer, "Restoration of motion-related signal loss and line-shape deterioration of proton MR spectra using the residual water as intrinsic reference," Magnetic Resonance in Medicine, vol. 46, pp. 395-400, aug 2001.

[107] J. Near, R. Edden, C. J. Evans, R. Paquin, A. Harris, and P. Jezzard, "Frequency and phase drift correction of magnetic resonance spectroscopy data by spectral registration in the time domain," Magnetic Resonance in Medicine, vol. 73, no. 1, pp. 44-50, 2015.

[108] B. C. Rowland, H. Liao, F. Adan, L. Mariano, J. Irvine, and A. P. Lin, "Correcting for Frequency Drift in Clinical Brain MR Spectroscopy," Journal of Neuroimaging, vol. 27, pp. 23-28, jan 2017.

[109] K. I. Marro, D. Lee, E. G. Shankland, C. Mark Mathis, C. E. Hayes, S. D. Friedman, and M. J. Kushmerick, "Quantitative In vivo magnetic resonance spectroscopy using synthetic signal injection," PLoS ONE, vol. 5, no. 12, p. 15166, 2010.

[110] C. Gasparovic, T. Song, D. Devier, H. J. Bockholt, A. Caprihan, P. G. Mullins, S. Posse, R. E. Jung, and L. A. Morrison, "Use of tissue water as a concentration reference for proton spectroscopic imaging," Magnetic Resonance in Medicine, vol. 55, pp. 1219-1226, jun 2006. 
[111] Y. Ishihara, A. Calderon, H. Watanabe, K. Okamoto, Y. Suzuki, K. Kuroda, and Y. Suzuki, "A precise and fast temperature mapping using water proton chemical shift," Magnetic Resonance in Medicine, vol. 34, pp. 814-823, dec 1995.

[112] J. Pauly, "Field Maps," Stanford Lecture Notes, pp. 1-7, 2005.

[113] G. Reynolds, M. Wilson, A. Peet, and T. N. Arvanitis, "An algorithm for the automated quantitation of metabolites in in vitro NMR signals," Magnetic Resonance in Medicine, vol. 56, no. 6, pp. 1211-1219, 2006.

[114] M. Wilson, G. Reynolds, R. A. Kauppinen, T. N. Arvanitis, and A. C. Peet, "A constrained least-squares approach to the automated quantitation of in vivo $1 \mathrm{H}$ magnetic resonance spectroscopy data," Magnetic Resonance in Medicine, vol. 65, pp. 1-12, jan 2011.

[115] C. L. Lawson and R. J. Hanson, Solving Least Squares Problems. SIAM, 1995.

[116] A. Naressi, C. Couturier, J. M. Devos, M. Janssen, C. Mangeat, R. De Beer, and D. Graveron-Demilly, "Java-based graphical user interface for the MRUI quantitation package," Magnetic Resonance Materials in Physics, Biology and Medicine, vol. 12, no. 2-3, pp. 141-152, 2001.

[117] D. Stefan, F. D. Cesare, A. Andrasescu, E. Popa, A. Lazariev, E. Vescovo, O. Strbak, S. Williams, Z. Starčuk, M. Cabanas, D. Van Ormondt, and D. Graveron-Demilly, "Quantitation of magnetic resonance spectroscopy signals: The jMRUI software package," Measurement Science and Technology, vol. 20, no. 10, 2009.

[118] R. De Beer and D. Van Ormondt, Analysis of NMR Data Using Time Domain Fitting Procedures, pp. 201-248. Berlin, Heidelberg: Springer Berlin Heidelberg, 1992.

[119] H. Barkhuijsen, R. De Beer, and D. Van Ormondt, "Improved algorithm for noniterative time-domain model fitting to exponentially damped magnetic resonance signals," Journal of Magnetic Resonance (1969), vol. 73, pp. 553-557, jul 1987. 
[120] S. Vanhuffel, H. Chen, C. Decanniere, and P. Vanhecke, "Algorithm for Time-Domain NMR Data Fitting Based on Total Least Squares," Journal of Magnetic Resonance, Series A, vol. 110, pp. 228-237, oct 1994.

[121] H. Barkhuijsen, R. De Beer, W. Bovée, and D. Van Ormondt, "Retrieval of frequencies, amplitudes, damping factors, and phases from time-domain signals using a linear leastsquares procedure," Journal of Magnetic Resonance (1969), vol. 61, pp. 465-481, feb 1985.

[122] L. Vanhamme, van den Boogaart A, S. Van Huffel, A. van den Boogaart, and S. Van Huffel, "Improved Method for Accurate and Efficient Quantification of MRS Data with Use of Prior Knowledge," Journal of Magnetic Resonance, vol. 129, pp. 35-43, nov 1997.

[123] J. W. C. van der Veen, R. de Beer, P. R. Luyten, and D. van Ormondt, "Accurate quantification ofin vivo31P NMR signals using the variable projection method and prior knowledge," Magnetic Resonance in Medicine, vol. 6, pp. 92-98, jan 1988.

[124] J. E. J. E. Dennis, R. B. Schnabel, and Society for Industrial and Applied Mathematics., Numerical methods for unconstrained optimization and nonlinear equations. Society for Industrial and Applied Mathematics (SIAM, 3600 Market Street, Floor 6, Philadelphia, PA 19104), 1996.

[125] Z. Starčuk and J. Starčuková, "Quantum-mechanical simulations for in vivo MR spectroscopy: Principles and possibilities demonstrated with the program NMRScopeB," Analytical Biochemistry, vol. 529, pp. 79-97, 2017.

[126] Z. Starčuk, J. Starčuková, O. Štrbak, and D. Graveron-Demilly, "Simulation of coupled-spin systems in the steady-state free-precession acquisition mode for fast magnetic resonance (MR) spectroscopic imaging," Measurement Science and Technology, vol. 20, no. 10, 2009.

[127] H. Ratiney, Y. Coenradie, S. Cavassila, D. Van Ormondt, and D. Graveron-Demilly, "Time-domain quantitation of1H short echo-time signals: Background accommoda- 
tion," Magnetic Resonance Materials in Physics, Biology and Medicine, vol. 16, no. 6, pp. 284-296, 2004.

[128] H. Ratiney, M. Sdika, Y. Coenradie, S. Cavassila, D. Van Ormondt, and D. GraveronDemilly, "Time-domain semi-parametric estimation based on a metabolite basis set," NMR in Biomedicine, vol. 18, no. 1, pp. 1-13, 2005.

[129] W. K. J. Renema, H. E. Kan, B. Wieringa, and A. Heerschap, "In vivo magnetic resonance spectroscopy of transgenic mouse models with altered high-energy phosphoryl transfer metabolism," NMR in Biomedicine, vol. 20, no. 4, pp. 448-467, 2007.

[130] D. M. Sima and S. Van Huffel, "Separable nonlinear least squares fitting with linear bound constraints and its application in magnetic resonance spectroscopy data quantification," Journal of Computational and Applied Mathematics, vol. 203, pp. 264-278, jun 2007.

[131] S. W. Provencher, "Estimation of metabolite concentrations from localized in vivo proton NMR spectra," Magnetic Resonance in Medicine, vol. 30, pp. 672-679, dec 1993.

[132] S. W. Provencher, "Automatic quantitation of localized in vivo1H spectra with LCModel," NMR in Biomedicine, vol. 14, pp. 260-264, jun 2001.

[133] D. W. Marquardt, "An Algorithm for Least-Squares Estimation of Nonlinear Parameters," Journal of the Society for Industrial and Applied Mathematics, vol. 11, pp. 431441, jun 1963.

[134] B. Soher, D. Semanchuk, D. Todd, J. Steinberg, and K. Young, "VeSPA: Integrated applications for RF pulse design, spectral simulation and MRS data analysis," Proceedings of the 19th Annual Meeting ISMRM, vol. 19, no. 19, p. 1410, 2011.

[135] G. B. Matson, "An integrated program for amplitude-modulated RF pulse generation and re-mapping with shaped gradients," Magnetic Resonance Imaging, vol. 12, pp. 1205-1225, jan 1994. 
[136] B. J. Soher, K. Young, A. Bernstein, Z. Aygula, and A. A. Maudsley, "GAVA: Spectral simulation for in vivo MRS applications," Journal of Magnetic Resonance, vol. 185, pp. 291-299, apr 2007.

[137] J. Kornak, K. Young, B. J. Soher, and A. A. Maudsley, "Bayesian k-Space-Time Reconstruction of MR Spectroscopic Imaging for Enhanced Resolution," Ieee Transactions on Medical Imaging, vol. 29, no. 7, pp. 1333-1350, 2010.

[138] K. Young, B. J. Soher, and A. A. Maudsley, "Automated spectral analysis II: Application of wavelet shrinkage for characterization of non-parameterized signals," Magnetic Resonance in Medicine, vol. 40, pp. 816-821, dec 1998.

[139] B. J. Soher, K. Young, V. Govindaraju, and A. A. Maudsley, "Automated spectral analysis III: Application to in vivo proton MR spectroscopy and spectroscopic imaging," Magnetic Resonance in Medicine, vol. 40, pp. 822-831, dec 1998.

[140] S. Smith, T. Levante, B. Meier, and R. Ernst, "Computer Simulations in Magnetic Resonance. An Object-Oriented Programming Approach,” 1994.

[141] R. Edden, N. A. J. Puts, A. D. Harris, P. B. Barker, and C. J. Evans, "Gannet: A batch-processing tool for the quantitative analysis of gamma-aminobutyric acid-edited MR spectroscopy spectra," Journal of Magnetic Resonance Imaging, vol. 40, no. 6, 2014.

[142] R. Simpson, G. A. Devenyi, P. Jezzard, T. J. Hennessy, and J. Near, "Advanced processing and simulation of MRS data using the FID appliance (FID-A) - An open source, MATLAB-based toolkit," Magnetic Resonance in Medicine, vol. 77, pp. 23-33, jan 2017.

[143] R. Kreis and C. S. Bolliger, "The need for updates of spin system parameters, illustrated for the case of g -aminobutyric acid," NMR in Biomedicine, no. May, pp. 1401$1403,2012$. 
[144] D. D'Alessandro, Introduction to Quantum Control and Dynamics, vol. 13 of Chapman $\& 6$ Hall/CRC Applied Mathematics \& Nonlinear Science. Chapman and Hall/CRC, aug 2007.

[145] P. Doria, T. Calarco, and S. Montangero, "Optimal control technique for many-body quantum dynamics," Physical Review Letters, vol. 106, no. 19, 2011.

[146] J. P. Palao and R. Kosloff, "Optimal control theory for unitary transformations," Physical Review A - Atomic, Molecular, and Optical Physics, vol. 68, p. 13, dec 2003.

[147] J. P. Palao and R. Kosloff, "Molecular Quantum Computing by an Optimal Control Algorithm for Unitary Transformations," Physical Review Letters, vol. 89, no. 3, p. 188301, 2002.

[148] N. Khaneja, T. Reiss, C. Kehlet, T. Schulte-Herbrüggen, and S. J. Glaser, "Optimal control of coupled spin dynamics: Design of NMR pulse sequences by gradient ascent algorithms," Journal of Magnetic Resonance, vol. 172, pp. 296-305, feb 2005.

[149] S. Machnes, U. Sander, S. J. Glaser, P. De Fouquières, A. Gruslys, S. Schirmer, and T. Schulte-Herbrüggen, "Comparing, optimizing, and benchmarking quantum-control algorithms in a unifying programming framework," Physical Review A - Atomic, Molecular, and Optical Physics, vol. 84, no. 2, p. 22305, 2011.

[150] T. Schulte-Herbrüggen, A. Spörl, N. Khaneja, and S. J. Glaser, "Optimal controlbased efficient synthesis of building blocks of quantum algorithms: A perspective from network complexity towards time complexity," Physical Review A - Atomic, Molecular, and Optical Physics, vol. 72, p. 042331, oct 2005.

[151] F. F. Floether, P. De Fouquieres, and S. G. Schirmer, "Robust quantum gates for open systems via optimal control: Markovian versus non-Markovian dynamics," New Journal of Physics, vol. 14, 2012.

[152] R. Blatt and D. Wineland, "Entangled states of trapped atomic ions," jun 2008. 
[153] D. Leibfried, R. Blatt, C. Monroe, and D. Wineland, "Quantum dynamics of single trapped ions," mar 2003.

[154] J. J. García-Ripoll, P. Zoller, and J. I. Cirac, "Coherent control of trapped ions using off-resonant lasers," Physical Review A - Atomic, Molecular, and Optical Physics, vol. 71 , p. 062309 , jun 2005.

[155] R. Riek, G. Wider, K. Pervushin, and K. Wüthrich, "Polarization transfer by crosscorrelated relaxation in solution NMR with very large molecules.," Proceedings of the National Academy of Sciences of the United States of America, vol. 96, pp. 4918-23, apr 1999.

[156] R. Xu, Y. Yan, Y. Ohtsuki, Y. Fujimura, and H. Rabitz, "Optimal control of quantum non-Markovian dissipation: Reduced Liouville-space theory," The Journal of Chemical Physics, vol. 120, pp. 6600-6608, apr 2004.

[157] H. Jirari and W. Pötz, "Quantum optimal control theory and dynamic coupling in the spin-boson model," Physical Review A - Atomic, Molecular, and Optical Physics, vol. 74, p. 022306, aug 2006.

[158] S. Conolly, D. Nishimura, and A. Macovski, "Optimal Control Solutions to the Magnetic Resonance Selective Excitation Problem," IEEE Transactions on Medical Imaging, vol. 5, pp. 106-115, jun 1986.

[159] J. Mao, T. H. Mareci, K. N. Scott, and E. R. Andrew, "Selective inversion radiofrequency pulses by optimal control," Journal of Magnetic Resonance (1969), vol. 70, pp. 310-318, nov 1986.

[160] D. Rosenfeld and Y. Zur, "Design of adiabatic selective pulses using optimal control theory," Magnetic Resonance in Medicine, vol. 36, pp. 401-409, sep 1996.

[161] T. E. Skinner, T. O. Reiss, B. Luy, N. Khaneja, and S. J. Glaser, "Application of optimal control theory to the design of broadband excitation pulses for high-resolution NMR," Journal of Magnetic Resonance, vol. 163, pp. 8-15, jul 2003. 
[162] T. E. Skinner, T. O. Reiss, B. Luy, N. Khaneja, and S. J. Glaser, "Reducing the duration of broadband excitation pulses using optimal control with limited RF amplitude," Journal of Magnetic Resonance, vol. 167, pp. 68-74, mar 2004.

[163] K. Kobzar, T. E. Skinner, N. Khaneja, S. J. Glaser, and B. Luy, "Exploring the limits of broadband excitation and inversion pulses, J," Magn. Reson., vol. 170, pp. 236-243, oct 2004 .

[164] H. J. Hogben, M. Krzystyniak, G. T. Charnock, P. J. Hore, and I. Kuprov, "Spinach - A software library for simulation of spin dynamics in large spin systems," Journal of Magnetic Resonance, vol. 208, pp. 179-194, feb 2011.

[165] S. G. Schirmer and P. De Fouquieres, "Efficient algorithms for optimal control of quantum dynamics: The Krotov method unencumbered," New Journal of Physics, vol. $13,2011$.

[166] T. O. Levante, T. Bremi, and R. R. Ernst, "Pulse-sequence optimization with analytical derivatives. Application to deuterium decoupling in oriented phases," Journal of Magnetic Resonance - Series A, vol. 121, no. 2, pp. 167-177, 1996.

[167] R. H. Byrd, P. Lu, J. Nocedal, and C. Zhu, "A Limited Memory Algorithm for Bound Constrained Optimization," SIAM Journal on Scientific Computing, vol. 16, pp. 11901208, sep 1995.

[168] W. Zaaraoui, L. Fleysher, R. Fleysher, S. Liu, B. J. Soher, and O. Gonen, "Human brain-structure resolved T2relaxation times of proton metabolites at 3 Tesla," Magnetic Resonance in Medicine, vol. 57, pp. 983-989, jun 2007.

[169] N. A. Puts, P. B. Barker, and R. A. Edden, "Measuring the longitudinal relaxation time of GABA in vivo at 3 Tesla," Journal of Magnetic Resonance Imaging, vol. 37, pp. 999-1003, apr 2013.

[170] R. A. Edden, D. Bonekamp, M. A. Smith, P. Dubey, and P. B. Barker, "Proton MR spectroscopic imaging of the medulla and cervical spinal cord," Journal of Magnetic Resonance Imaging, vol. 26, pp. 1101-1105, oct 2007. 
[171] D. C. Shungu and J. D. Glickson, "Sensitivity and localization enhancement in multinuclear in vivo NMR spectroscopy by outer volume presaturation," Magnetic Resonance in Medicine, vol. 30, pp. 661-671, dec 1993.

[172] I. Y. Choi, I. Tkac, and R. Gruetter, "Single-shot, three-dimensional 'non-echo' localization method for in vivo NMR spectroscopy," Magnetic Resonance in Medicine, vol. 44, pp. 387-394, sep 2000.

[173] A. Henning, A. Fuchs, J. B. Murdoch, and P. Boesiger, "Slice-selective FID acquisition, localized by outer volume suppression (FIDLOVS) for1H-MRSI of the human brain at 7 T with minimal signal loss," NMR in Biomedicine, vol. 22, no. 7, pp. 683-696, 2009.

[174] A. Henning, M. Scharr, R. F. Schulte, B. Wilm, K. P. Pruessmann, and P. Boesiger, "SELOVS: Brain MRSI localization based on highly selective T1- and B1-insensitive outer-volume suppression at 3T," Magnetic Resonance in Medicine, vol. 59, pp. 40-51, jan 2008. 